\title{
ORDERED CERAMIC MEMBRANES
}

\section{Final Report}

\section{By}

Marc A. Anderson

Charles G. Hill, Jr.

Walter A. Zeltner

October 1991

Work Performed Under Contract No. AS07-86ID12626

For

U. S. Department of Energy

Office of Industrial Technologies

Washington, D.C.

By

University of Wisconsin-Madison

Madison, Wisconsin 


\section{DISCLAIMER}

This report was prepared as an account of work sponsored by an agency of the Inited States Govermment. Neither the United States Government nor any agency thereof nor ant of their employees. makes any warranty. express or implied. or assumes any legal lability or responsibilin for the accuracy, completeness. or usefulness of any information, apparatus, product. or process disclosed, or represents that its use would not infringe privately owned rights. Reference herein to any specific commercial product, process, or service by trade name, trademark, manufacturer. or otherwise does not necessarily constitute or imply its endorsement, recommendation, or favoring by the Linited States Government or any agency thereof. The views and opinions of authors ex. pressed herein do not necessarily state or reflect those of the United States Government or any agency thereof.

This report has been reproduced directly from the best available copy.

Available to DOE and DOE contractors from the Office of Scientific and Technical Information. P.O. Box 62. Oak Ridge. TN 37831: prices available from 161515768401 . FTS 626-8401.

Available to the public from the National Technical Information Service. U. S. Department of Commerce. 5285 Port Royal Rd.. Springfield, VA 22161. 
DOE/ID/12626--T3

DE92 002945

\section{ORDERED CERAMIC MEMBRANES}

FINAL REPORT

Prof. Marc A. Anderson

Prof. Charles G. Hill, Jr.

Dr. Walter A. Zeltner

October 1991

Work Performed Under Contract DE-AS07-86ID12626

Prepared for

U. S. Department of Energy

Idaho Operations office, Idaho Falls, ID

Sponsored by the Office of the Assistant Secretary

for Conservation and Renewable Energy

Office of Industrial Technologies

Washington D.C.

Prepared by

Water Chemistry Program

University of Wisconsin - Madison

Madison, Wiscons in 53706 


\section{Ordered Ceramic Membranes - Final Report}

M.A. Anderson, I.A. Zeltner, and C.G. Hill, Jr.

\section{ABSTRACT}

Ceramic membranes have been formed from colloidal sols coated on porous clay supports. These supported membranes have been characterized in terms of their permeabilities and permselectivities to various aqueous test solutions. The thermal stabilities and pore structures of these membranes have been characterized by preparing unsupported membranes of the corresponding material and performing $\mathrm{N}_{2}$ adsorption-desorption and $\mathrm{X}$-ray diffraction studies on these membranes. To date, membranes have been prepared from a variety of oxides, including $\mathrm{TiO}_{2}, \mathrm{SiO}_{2}, \mathrm{ZrO}_{2}$, and $\mathrm{Al}_{2} \mathrm{O}_{3}$, as well as $\mathrm{Zr}-, \mathrm{Fe}-$, and $\mathrm{Nb}-$ doped $\mathrm{TiO}_{2}$. In many of these membranes pore diameters are less than $2 \mathrm{~nm}$, while in others the pore diameters are between 3 and $5 \mathrm{~nm}$. Procedures for fabricating porous clay supports with reproducible permeabilities for pure water are also discussed. 
I. Executive Summary . . . . . . . . . . . . . . . . . . . . . . . . 1

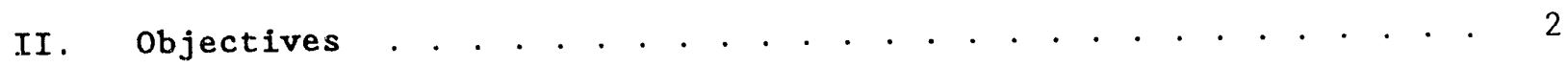

III. Supports .

A. General Considerations . . . . . . . . . . . . . . . . . . 3

B. Concerns . . . . . . . . . . . . . . . . . . . . . . . . . . . . . 44

1. Use of Commercial Supports . . . . . . . . . . . . . . . 4

2. Fabrication Technique . . . . . . . . . . . . . . 4

3. Shape of Support . . . . . . . . . . . . . . . . . . 5

4. Composition of Support . . . . . . . . . . . . . . . 6

a. Fabrication of Clay Supports . . . . . . . . . 7

b. Preparation of Supports with Reproducible

c. Preparation of Supports with Smoother
Outer Surfaces . . . . . . . . . . . . 9

C. Future Outlook . . . . . . . . . . . . . . . . . . . . . . 11

IV. Unsupported Membranes . . . . . . . . . . . . . . . . . . . . . . 12

A. Ceramic Membranes Formed from Conventional Sols . . . . . $\quad 15$

1. Alumina . . . . . . . . . . . . . . . . . . . . . . . . . 17

a. Experimental Method . . . . . . . . . . . 17

b. Effects of Sintering Conditions on the Properties of Unsupported Membranes . . . 19

1. Effects of Sintering at $500^{\circ} \mathrm{C} . . .+. \quad 19$

2. Effects of Varying the Sintering Temperature . . . . . . . . . 23

c. Effects of Solid Concentration in the Sol on the Properties of Unsupported Membranes . 24

d. Effects of Phosphate Addition on the Properties of Unsupported Membranes . . . 25

e. Summary . . . . . . . . . . . . . . . . . . 28 
a. Experimental Method ............. 31

b. Effects of the Solvent Employed on the Properties of Unsupported Membranes . . . 32

c. Effects of Reactant Concentrations on the Properties of Unsupported Membranes . . . 33

d. Effects of Solid Concentration on the Properties of Unsupported Membranes . . . 34

e. Effects of Sintering Temperature on the Properties of Unsupported Membranes . . . 37

f. Summary . . . . . . . . . . . . . . . . 37

3. Titania . . . . . . . . . . . . . . 41

a. Experimental Method - Polymeric Sols . . . . . 41

b. Experimental Method - Par -iculate Sols . . . . 44

c. Effects of Hydrolysis and Peptization Conditions on the Particle Size of Titania Sols . . . . . . . . . . 46

d. Control of Particle Packing Density During Gelation... . . . . . . . 50

1. Formation of Dense Gels . . . . . . . 51

2. Formation of More Open Gels .. . . . . . 55

3. Conclusions . . . . . . . . . . . 58

e. Effects of Sintering Conditions on the Properties of Unsupported Membranes . . . 59

f. Effects of Phosphate Addition on the Properties of Unsupported Membranes . . 67

g. Summary . . . . . . . . . . . . . . . 69

4. Other Materials . . . . . . . . . . . . . . 73

a. Zirconia . . . . . . . . . . . . . . 73

b. Mixtures of Titania and Zirconia . . . . . . . 76

c. Other Mixed Oxides . . . . . . . . . . . . 78

d. Summary . . . . . . . . . . . . . . . 80 
B. Ceramic Membranes Formed from Nanoparticulate Sols . . . 81

1. Solubility Control . . . . . . . . . . . . . . . . 81

a. Explanation of the Technique . . . . . . . . 81

b. Detailed Procedures . . . . . . . . . . . 86

c. Charge Development on Particles. . . . . . . 87

d. Thermal Stability of Unsupported Membranes . . 91

2. Steric Hindrance . . . . . . . . . . . . . . . . . 96

a. Detailed Procedures . . . . . . . . . . 96

b. Effects of Reactant Concentration on

c. Thermal Stability of Unsupported Membranes . . 103

1. Single Phase Membranes . . . . . . . 103

2. Mixed Oxide Membranes . . . . . . . 108

V. Fabrication of Supported Membranes . . . . . . . . . . . . . . . 113

A. Coating Techniques . . . . . . . . . . . . . . . . . . . . 113

B. Drying Problems . . . . . . . . . . . . . . . . . . . . . . 116

VI. Properties of Supported Membranes . . . . . . . . . . . . . . . . 117

A. Permeate Flux Measurements . . . . . . . . . . . . . . . . 117

1. Coating Material and Number of Dippings . . . . . . . 118

2. Dipping and Firing Treatments . . . . . . . . . . . . 120

3. $\mathrm{pH}$ of Feed Stream . . . . . . . . . . . . . . . . . 124

a. Stability of Supported Membranes . . . . . . 125

1. Procedures . . . . . . . . . . . 125

2. Results................. . . 129

3. Conclusions . . . . . . . . . . . . 133

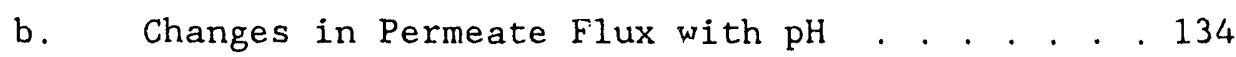


4. Time of Exposure to Test Stream . . . . . . . . . . 137

5. Temperature of the Feed Stream . . . . . . . . . . 140

6. Pressure of the Feed Stream . . . . . . . . . . . 142

B. Permselectivity Measurements . . . . . . . . . . . 143

1. Procedures Employed ................. 143

2. Permselectivities of Supported Alumina Membranes . . 144

3. Effects of Phosphate Treatment on the Properties

of Supported Alumina Membranes . . . . . . 146

4. Permselectivities of Supported Titania Membranes . . 151

C. Regeneration of Membranes . . . . . . . . . . . . . 152

VII. Conclusions . . . . . . . . . . . . . . . . . . . . . . . 154

A. Supports . . . . . . . . . . . . . . . . . . 154

B. Unsupported Membranes . . . . . . . . . . . . . . . . 154

1. Techniques for Synthesizing Sols . . . . . . . . . 154

2. Effects of Adding Phosphate............ 156

3. Thermal Stability of Membranes . . . . . . . . . 158

C. Fabrication and Properties of Unsupported Membranes . . . 158

VIII. References . . . . . . . . . . . . . . . . . . . . . 161 


\section{Executive Summary}

One major goal of this project has been the preparation of ceramic membranes with a variety of compositions and morphologies. However, a goal of equal importance has been to characterize the behavior of these membranes when they are incorporated in modules that are designed to perform separations of commercial interest. In order to fulfill these two goals, our research has focused on several areas: preparation of porous supports, synthesis and characterization of unsupported ceramic membranes, fabrication of modules in which the ceramic membranes are bonded to porous supports, and characterization of the performance of the resulting modules.

Techniques have been developed to prepare porsus clay supports with reproducible permeabilities. These test-tube shaped supports can be inserted in a test apparatus to determine the permselectivities of the supported ceramic membranes. We are still attempting to identify commercial sources of porous supports which would be more appropriate for applications of interest. Ceramic membranes with several compositions $\left(\mathrm{Al}_{2} \mathrm{O}_{3}, \mathrm{TiO}_{2}, \mathrm{SiO}_{2}\right.$, and $\mathrm{ZrO}_{2}$, as well as mixed oxides) have been synthesized as either conventional membranes (pore diameters in the $3-5 \mathrm{~nm}$ range) or membranes formed from nanosized particles (pore diameters of less than $2 \mathrm{~nm}$ ). Modules which incorporate ceramic membranes formed from conventional sols display very good permselectivities for applications involving ultrafiltration. Modules which incorporate membranes formed from nanoparticles have potential applications in reverse osmosis and gas phase separations. To date, however, we have been unable to study these particular applications of the nanoparticle membranes because of serious problems with cracks which form in these membranes during fabrication. Alternative methods of fabricating supported nanoparticulate membranes may be able to overcome the problem of crack formation. 


\section{Objectives}

The purpose of this research is to produce ceramic membranes with specified pore sizes, pore size distributions anci surfact properties by controlling the suspension and interfacial chemistries of the precursor sols. In order to attain these ends, our major objectives have been:

1. to prepare supports for the ceramic membranes which will permit the properties of the supported membranes to be studied on a laboratory scale;

2. to define the effects of several variables (e.g., reactant concentrations, amount of added phosphate, $\mathrm{pH}$, reaction temperature, etc.) on the preparation and properties of the sols used to fabricate unsupported and supposted ceramic membranes;

3. to define the effects of ramp rate, firing temperature and dwell time on the properties of unsupported membranes;

4. to fabricate different types of unsupported membranes composed of various materials (e.g., $\mathrm{Al}_{2} \mathrm{O}_{3}, \mathrm{TiO}_{2}, \mathrm{SiO}_{2}$ and doped membranes) in which the size of the primary particles is controlled, in particular to prepare ceramic membranes from sols composed of particles with characteristic dimensions in the nanometer range (nanoparticles);

5. to prepare supported membranes and characterize their permselective properties in laboratory scale experiments;

6. to develop techniques suitable for the fabrication of commercial membrane units. 
III. Supports

\section{A. General Considerations}

Ceramic membranes with pore sizes that are small enough to be useful for micro- and ultrafiltration applications are quite fragile. In order to employ these materials in commercially viable systems, the membranes must be coated onto a strong, rigid, porous support. Along with many other requirements that the final system must meet, both the support and the membrane must be able to withstand the operating pressure of the solvent and the cleaning procedures used to eliminate any fouling that occurs in the system.

In addition, the pore size of the support must be larger than, but somewhat comparable to, the size of the particles in the sol that will be coated on the support. The ideal ceramic membrane would be a crack-free layer of particles that is very thin in order to maximize the permeability of the solvent through the membrane. If the pores of the support are much larger than the particles to be coated, the particles of the sol will readily move through the support without forming a thin coating at or near the surface of the support. Even if a thin coating can be formed on the support, that coating must be able to withstand the operating pressure of the solvent without being forced back into the support. 
B. Concerns

1. Use of Commercial Supports

Early in this project, considerable effort was devoted to preparing suitable porous supports on which ceramic membranes could be coated. Once coated, the resulting modules could then be tested to determine their permeabilities and permselectivities. This effort was necessary because initial attempts to utilize commercially available porous alumina disks as supports were unsuccessful. The alumina disks were not strong enough to withstand the pressures developed in the test apparatus. Other porous supports (e.g., Ceraver alumina tubes) might have been strong enough for use but were not readily available.

\section{Fabrication Technique}

Two techniques were considered for fabricating supports: slip casting and injection molding (extrusion). While extrusion is commonly used by industry to prepare porous supports, we had neither the equipment nor the expertise to readily utilize this approach. Consequently, we prepared our porous supports by slip casting.

Slip casting is a process in which a powdered material is suspended in a suitable liquid vehicle (in our case, water). This suspension must be stable (particles must not settle out of the sol during the time that slip casting occurs). In order to fulfill this requirement successfully, the colloid chemistry of the sol must be controlled carefully, an area in which we do have 
considerable experience. Once a stable sol is prepared, it is poured into an absorbent mold which draws off most of the liquid. The casting is left to dry in place, at which time it must be strong enough to be handled for firing.

The gypsum molds that were employed were prepared from a mix of two parts by weight piaster and one part by weight water. After the plaster powder was added to the water, the inixture was allowed to settle for a couple of minutes. This mixture was then poured carefully into a container mold (designed to give a gypsum mold of the desired size and shape) in order to minimize the formation of air bubbles. The mixture was left in the container mold until the plaster was almost completely dry. At that time, the container mold was removed, and the working gypsum mold was air dried for two more days before use. (If the gypsum mold is heated at this point to speed the drying process, it will lose more water than is desirable and become very powdery.)

\section{Shape of Support}

Alumina disks were tested initially but were not strong enough to withstand the pressures generated in our test apparatus. We then opted to prepare the slip cast supports in a "finger" or test-tube shape. In this configuration, the supports could be easily dipped into sols of the desired materials for fabrication of supported membranes. Membranes placed on the outside of such supports would show optimun compressive strength and thus would have less tendency to shear off the supports during filtration. While test-tube shaped supports do not provide optimum filtration efficiency due to their low surface area to volume ratio, the studies conducted for this project were performed at the laboratory scale and were designed to characterize the 
properties of supported ceramic membranes rather than to optimize their performance. The test-tube shaped supports worked very well for these studies.

4. Composition of Support

Supports have been prepared from both clay mixtures and alumina, with most of our early work focused on preparing and characterizing alumina supports. (Details of this work are given in ref. 1, pp. 44-57 and ref. 2, pp. 6-8.) Although the initial alumina supports had strengths and permeabilities that were high enough to be useful, these supports could not be fabricated with reproducible permeabilities. Further work did result in the fabrication of alumina supports that had permeabilities which varied by about 58 , but these supports were more powdery and weaker than similar clay supports. In addition, significant problems were encountered in coating alumina ceramic membranes on alumina supports. In some cases, the precursor sol appeared to completely infiltrate the support without forming a membrane. Even when visual observation indicated that coatings appeared to form on supports, scanning electron microscopy (SEM) studies did not reveal the formation of distinguishable membranes on these supports. While this problem might have been solved by incorporating an intermediate "sandwich" layer of alumina on the support, an extra fabrication step would have been required. Once it was found that alumina and titania membranes would adhere directly to clay supports, attempts to prepare alumina supports were terminated. 
a. Fabrication of Clay Supports

Formulations and experimental protocols were developed for preparing clay supports with reproducible characteristics $[2,3]$. The clay formulation used originally was 4 parts water, 2 parts coarse clay and 1 part fine clay by weight. Among the coarse clays utilized were AP Green, Yellow Banks, and Hawthorne Bond, while OM-4 ball clay was the fine clay used exclusively in this project. (With the exception of the data in Table I, all permeate flux and permselectivity data presented in this report were obtained from supports fabricated with AP Green clay.) After being slip cast, the supports were air dried for one day, during which time they released from the mold. The supports were then fired at $4^{\circ} \mathrm{C} / \mathrm{m}$ in to $1000^{\circ} \mathrm{C}$ and held at that temperature for $30 \mathrm{~min}$. They were then cooled to room temperature overnight. Although SEM studies suggested that these clay supports had pore sizes of a few microns, mercury porosimetry measurements indicated that a peak in the incremental pore volume was present at ca. 25 microns. The reason for this discrepancy is not known.

b. Preparation of Supports with Reproducible Characteristics

While these clay supports provided acceptable surfaces for coating with membranes, reproducibility among supports (as measured by the permeation rate of water) was very poor. If five supports were prepared in different molds at the same time and then fired, measured fluxes could vary by a factor of two. One reason for this variability is that the supports do not have a uniform height and thickness. We corrected for this variation by estimating the exposed surface area of the support and dividing measured permeate fluxes by 
this surface area to obtain corrected support permeation rates. High fluxes are likely to occur when the supports have many large pores. Thus, we needed to prepare supports with more reproducible, uniform flow properties.

For the clay supports, several formulations based on different coarse clays were prepared and tested. In all cases, sodium carbonate $(0.5-18$ by weight of $0.5 \mathrm{M}$ solution) and sodium silicate ( 0.38 by weight of a solution of 1 drop of commercial "water glass" in $10 \mathrm{~mL}$ of water) were added to the suspensions to minimize the formation of air bubbles. Another clay, bentonite, was also introduced in two formulations to increase the packing density of the particles and the viscosity of the suspensions. All suspensions used to prepare supports were hydrolyzed for at least 5 days, sieved, and hydrolyzed for at least one additional day after sieving. After this final hydroiysis, suspensions were slowly and carefully mixed to avoid the formation of air bubbles and then cast to form supports. Results of studies of the permeation rate of deionized water through these supports are shown in Table I.

Table I. Variations in permeate flux through supports prepared using different clay formulations.

\begin{tabular}{ccccc}
$\begin{array}{c}\text { Coarse Clay } \\
\text { Used }\end{array}$ & $\begin{array}{c}\text { Sieve } \\
\text { Mesh }\end{array}$ & $\begin{array}{c}\text { Sample } \\
\text { Size }\end{array}$ & $\begin{array}{c}\text { Water Flux } \\
\text { Average }\end{array}$ & $\begin{array}{c}\left.\mathrm{cm}^{3} / \mathrm{cm}^{2} / \mathrm{min}\right) \\
\text { Std. Deviation }\end{array}$ \\
\hline AP Green & 230 & 7 & 0.071 & 0.004 \\
AP Green (*) & 230 & 4 & 0.057 & 0.004 \\
AP Green & 170 & 7 & 0.067 & 0.004 \\
Hawthorne Bond & 230 & 4 & 0.044 & 0.007 \\
Yellow Banks (*) & 325 & 6 & 0.169 & 0.020
\end{tabular}
(*) Approximately 38 by weight of bentonite was also employed in this
formulation. 
In order to obtain reproducible behavior for the clay supports, the following factors must be noted. (1) Clay suspensions must hydrolyze for at least two days before casting. (2) During each cast, the suspension must be kept in the mold for exactly the same time (between 1 and $3 \mathrm{~min}$ ). Sieving the clay mixture to remove very coarse particles does not affect the behavior of the supports. (4) Addition of small quantities of bentonite to the clay mixture is not beneficial. (5) Our data indicate that $1000^{\circ} \mathrm{C}$ is the optimum firing temperature.

\section{c. Preparation of Supports with Smoother Outer Surfaces}

The initial permselectivity measurements performed on these coated supports gave very good results ( $>908$ rejection for 1 wt $q$ aqueous solutions of polyethylene glycol of molecular weight 1,000). Attempts to reproduce these results were unsuccessful, however. One factor that was considered as an explanation for these conflicting observations was the roughness of the surface of the support. (These discrepancies were later found to be due to the degree of hydration of the support rather than its surface roughness - see Section VI.A.4.)

Since these supports were coated on their outer surfaces, the roughness of the outer surface may have affected the physical integrity of the resultant coating and thus both the water permeability and the permselectivity of the composite material. SEM pictures of the outer surface of uncoated supports showed surface roughness on the order of microns. Several attempts were made to prepare supports with smoother outer surfaces. 
1. Three supports included in Sample 3 (see Table I) were hand polished and coated with 5 layers of alumina. However, the water permeabilities of these coated, hand polished supports and similar alumina membranes coated on non-polished supports fabricated in the same batch were indistinguishable. No further characterization tests were performed on these supports.

2. A clay suspension was ball-milled for $24 \mathrm{hr}$. Supports were then prepared from this suspension by slip casting. SEM pictures of the outer surfaces of these supports appeared virtually identical to the SEM pictures mentioned above. Hence, no further characterization tests were performed.

Another possible way to prepare smoother surfaces on the supports would be to coat the support, once it has been fabricated, with a second, thin layer of clay. However, this approach would introduce an extra fabrication step and was not pursued. (We stopped investigating this approach because, as a result of the studies undertaken to explain the conflicting permselectivity data that we obtained, we can now prepare supported alumina membranes with very good permselectivities without including this extra step.) In fact, by preparing clay supports as described above, we may provide a type of sandwich layer without needing an extra fabrication step. When the clay suspension is poured into the mold, the resulting arrangement of particles is determined by both gravity and capillary forces. For fine particles, capillary forces would be more important than the gravitational force. Thus, we would expect more fine material to be deposited on the outside of the supports than on the inside, leading to the natural formation of a sandwich layer.

The surface roughness of these clay supports did not appear to affect either the permeabilities or the permselectivities of the conposite supported membranes in these particular systems. However, the surface roughness of a 
support should have some effect on the physical integrity of a coating on that support. Thus, the roughness of the support should be considered as one factor that can influence the behavior of a composite supported membrane, unless experimental evidence demonstrates that surface roughness is not a problem.

\section{Future Outlook}

While porous clay supports have been used throughout this project to characterize the behavior of supported membranes formed from conventional sols, considerable effort was devoted in the last year of the project to preparing nanoparticulate sols. Although this project did not involve the preparation of supported ceramic membranes from sols containing ultra-fine particles, we expect that the pore sizes of these clay supports would be too large to be useful for supporting ultra-fine particles. Since we have found newer sources of porous supports as this project has progressed (e.g., thin alumina tubes fabricated by Technical Ceramics, Inc, Laurens, SC), we expect to utilize porous supports obtained from outside sources for any future work that requires characterization of ceramic membranes prepared from nanoparticulate sols. 


\section{Unsupported Membranes}

Although unsupported ceramic membranes are too thin and fragile to serve as useful filtration media themselves, they are fabricated for at least two reasons. (1) When crack-free unsupported membranes can be prepared, one gains confidence that similar membranes can be placed on supports without having the membrane crack. (2) Several important properties of the membranes can be determined only by using unsupported membranes. The exposed surface area, porosity and pore size distribution of a membrane can all be obtained from $\mathrm{N}_{2}$ BET adsorption studies, if enough membrane material is available. However, only very thin membranes, a few microns at most, are deposited on supports. Since these thin layers constitute only a very small fraction of the supported membrane system, BET measurements on such samples do not yield meaningful results. Hence only unsupported membranes are used for these studies. Large, crack-free membranes are not necessary for these measurements.

In all cases, these unsupported membranes were prepared by various modifications of the sol-gel process [4-9]. The synthetic method involves the controlled hydrolysis of metal alkoxides or metal salts to form either "particulate" or "polymeric" sols [10]. Unsupported membranes were formed by placing some of the sol in disposable polystyrene weighing dishes or Petri dishes and allowing the solvent in the sols to evaporate, often under controlled humidity conditions. The resulting xerogels were then sintered to obtain the final unsupported membrane. This process was also used to fabricate supported membranes. In this case, the sol was coated onto a support, carefully dried, and then fired to give a supported membrane. Figure 1 outlines the procedures used to prepare these materials. 

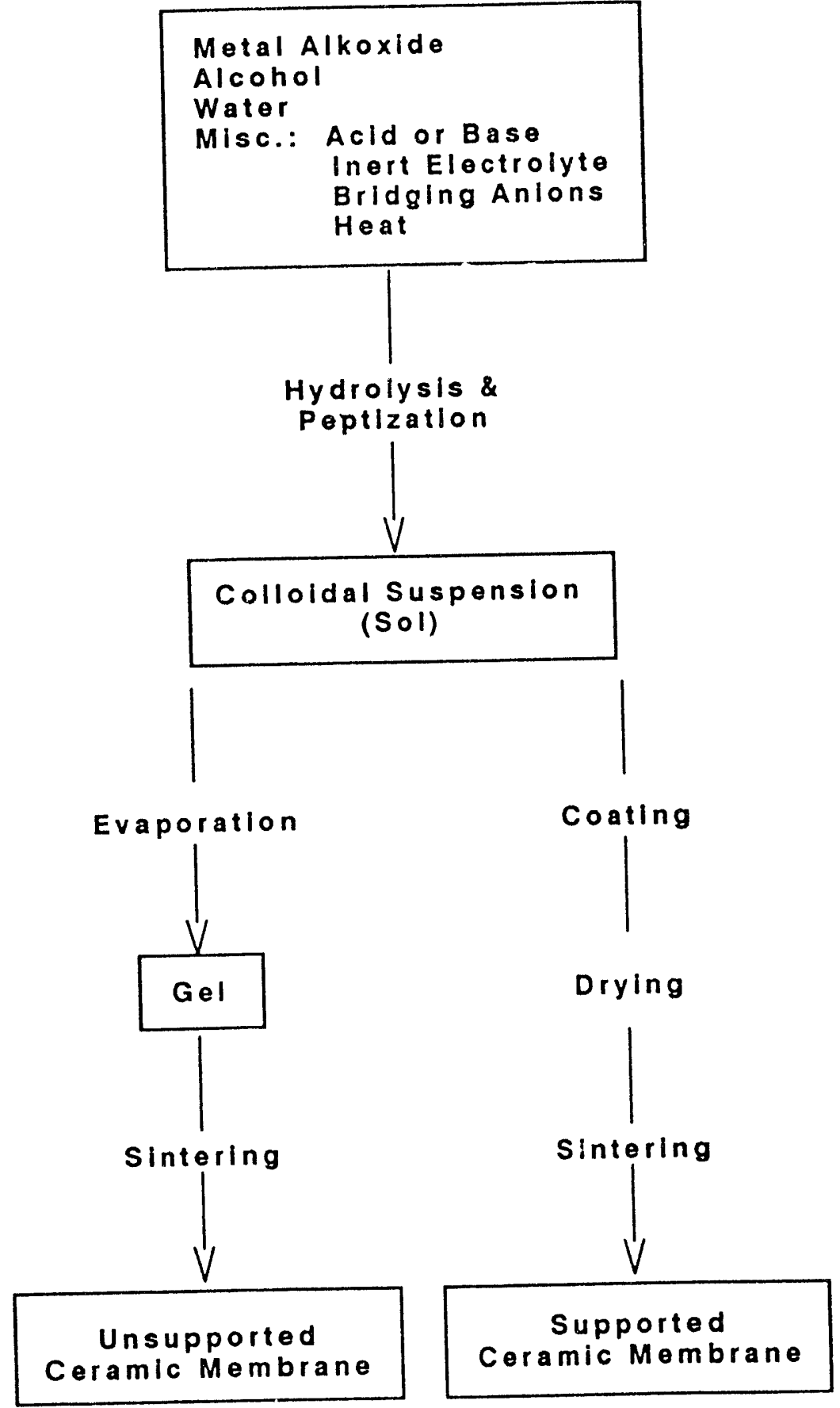

Figure 1. General procedure used to prepare unsupported and supported membranes. 
Fabrication of unsupported membranes has proceeded in two general directions distinguished by the sizes of the pores in the final membranes. The mean pore size is directly affected by the average particle size in the precursor sols. Initial investigations focused on preparing ceramic membranes with pore diameters in the range $3-5 \mathrm{~nm}$, corresponding to particle diameters of 15 nun or more [4-8]. While such membranes would be expected to be useful for ultrafiltration, they could not be expected to function well in applications involving either reverse osmosis or gas phase separations. However, data obtained during these studies led to the preparation of a second generation of ceramic membranes with pore diameters of $2 \mathrm{~nm}$ or less [9]. These membranes were fabricated from sols of so-called Q particles, particles with diameters less than ca. $5 \mathrm{~nm}$ which exhibit quantum effects in their optical spectra. There is considerable industrial interest in utilizing these second generation membranes for selected gas phase separations. However, we have not yet obtained commercial sponsors for research involving Ro applications. 
A. Ceramic Membranes Formed from Conventional Sols

Initial studies were directed at duplicating published syntheses of alumina membranes and increasing our knowledge about the colloid chemistry which controls the behavior of these systems [11]. As this information was obtained, efforts turned to the synthesis of titania membranes containing pores of similar size. $\mathrm{TiO}_{2}$ is quite interesting because of its photocatalytic properties. Some initial success has also been obtained in defining the conditions necessary for fabricating crack-free unsupported silica membranes [2]. However, these membranes shrank considerably during gelation. Attempts to replicate these syntheses during the past year have not succeeded. Hence it is clear that the factors that govern the behavior of this system were not completely elucidated in the earlier study. Zirconia membranes and several mixed oxide membranes $\left(\mathrm{ZrO}_{2} / \mathrm{TiO}_{2}, \mathrm{Fe}_{2} \mathrm{O}_{3} / \mathrm{TiO}_{2}\right.$ and $\mathrm{Nb}_{2} \mathrm{O}_{5} / \mathrm{TiO}_{2}$ ) have also been fabricated, although few characterization studies of these latter membranes have been performed.

Note that our synthesis of silica membranes was based on the use of alkaline hydrolysis to prepare the precursor sols. Most reported syntheses of silica membranes rely on acid hydrolysis. This difference reflects our belief that one can better control the mean pore size of the membranes if the precursor sols contain particles of reasonably uniform size. Acid hydrolysis of silicon alkoxides forms soluble polymeric species and ultimately leads to membranes with a network structure [10]. It would be difficult to control the structure of such membranes to give narrow pore size distributions (PSDs). Since alkaline hydrolysis of silicon alkoxides produces particles, the PSDs in the resulting membranes will be controlled primarily by the size distributions 
of the particles. If the particles exhibit narrow size distributions, then the resulting membranes would be expected to have narrow pore size distributions.

While particle size distributions have not been measured in most of our sols, we routinely determine PSDs for unsupported membranes obtained from these sols. For most sols of various compositions, these PSDs are relatively narrow. This fact allows us to infer that the particle size distributions in the precursor sols are also relatively narrow. Such considerations also explain our emphasis on using titania sols prepared with large water:alkoxide ratios; such sols can be expected to consist of titania particles rather than polymeric titania species.

In the following sections, we describe the studies performed on each of the three major types of unsupported membranes that we have prepared from conventional sols: alumina, titania and silica. The preparation of other materials is discussed briefly. 


\section{Alumina}

\section{a. Experimental Method}

All alumina systems were made from boehmite ( $\gamma$-A100H) sols prepared from aluminum tri-sec-butoxide (ATSB). To make these sols, a ca. 2 M solution of ATSB in 2-butanol was prepared to facilitate handling the otherwise viscous ATSB. This solution was quickly dripped into water at $80-85^{\circ} \mathrm{C}$ with vigorous stirring and allowed to react for $2 \mathrm{~h}$. (Heating the water above $80^{\circ} \mathrm{C}$ ensures the formation nf boehmite, a stable crystalline compound. At lower temperatures, one obcains an amorphous material that slowly transforms to bayerite. This material does not form gels that can be used for our purposes.)

After $2 \mathrm{~h}$, an aliquot of $1.6 \mathrm{M} \mathrm{HNO}_{3}$ was added to peptize the precipitate and stabilize the resulting suspension of boehmite. Heating and stirring were maintained for another hour. Subsequently, the reaction vessel was opened and the temperature was increased to drive off the alcohol. Initially, the temperature rose to $87^{\circ} \mathrm{C}$, the boiling point of the 2-butanol-water azeotrope. When most of the alcohol had boiled off, the temperature rose to $100^{\circ} \mathrm{C}$. At this stage, a condenser was positioned above the reaction vessel and the contents were refluxed overnight. The flask was then cooled to room temperature, and enough water was added to replace that lost by evaporation. Sols were stored in glass bottles until needed.

Table II summarizes the conditions employed in the initial hydrolyses used to prepare the boehmite sols. For most of these hydrolyses, the total concentration of $\mathrm{Al}$ was held at $0.5 \mathrm{M}$ while the initial acid concentration was varied between 0.015 and $0.048 \mathrm{M}$. In three hydrolyses, sodium nitrate was 
added as an inert electrolyte to determine the effect of increasing the ionic strength of the system on the properties of the resulting unsupported membranes. Phosphoric acid was added in two other experiments to assess the effect of the presence of a potential bridging anion on the properties of the membranes. These effects were assessed by noting the appearance of both the sol (cloudier sols would indicate that, on average, the particles were larger) and the xerogel.

Table II. Summary of hydrolysis conditions for preparing boehmite sols.

$\begin{array}{lcccccc}\text { Sample } & \begin{array}{c}{[\mathrm{A} 1]_{\text {total }}} \\ (\mathrm{M})\end{array} & \begin{array}{c}{\left[\mathrm{H}^{+}\right]^{\mathrm{a}}} \\ (\mathrm{M})\end{array} & \begin{array}{c}{\left[\mathrm{NaNO}_{3}\right]} \\ (\mathrm{M})\end{array} & \begin{array}{c}{\left[\mathrm{H}_{3} \mathrm{PO}_{4}\right]} \\ (\mathrm{M})\end{array} & \begin{array}{c}\text { Sol } \\ \text { Clarity }\end{array} & \begin{array}{c}\text { Xerogel } \\ \text { Appearance }\end{array} \\ \mathrm{H} 2 & 0.5 & 0.024 & - & - & 3 & \text { monolith } \\ \mathrm{H} 3 & 0.5 & 0.048 & - & - & 4 & \text { monolith } \\ \mathrm{H} 4 & 0.5 & 0.045 & - & - & 2 & \text { monolith } \\ \mathrm{H} 5 & 0.5 & 0.037 & - & - & 2 & \text { monolith } \\ \mathrm{H} 6 & 0.5 & 0.035 & 0.01 & - & 2 & \text { monolith } \\ \mathrm{H} 7 & 0.5 & 0.035 & 0.10 & - & 3 & \text { cracked } \\ \mathrm{H} 8 & 0.5 & 0.035 & 10^{-3} & - & 2 & \text { monolith } \\ \mathrm{H} 9 & 0.5 & 0.035 & - & 10^{-3} & 2 & \text { monolith } \\ \mathrm{H} 10 & 0.5 & 0.035 & - & 10^{-5} & 2 & \text { monolith } \\ \mathrm{H} 11 & 0.5 & 0.015 & - & - & 3 & \text { cracked } \\ \mathrm{H} 12 & 0.5 & - & 0.10 & - & \text { opaque } & \text { cracked } \\ \mathrm{H} 13 & 1.0 & 0.030 & - & - & 5 & \text { monolith }\end{array}$

a This value refers to the initial acid concentration immediately after adding acid to the sol. The final or equilibrium concentration is much lower since $\mathrm{H}^{+}$reacts with the boehmite particles.

b $\quad 1=$ clear; 5 = very cloudy

c Gel formed before completion of hydrolysis.

Monoliths are xerogeis that, visually, are completely crack-free.

For permselective applications of these alumina membranes, one would prefer to use the sols containing the smallest particles. Thus, the clearest sols that produced monolithic xerogels represented the most promising precursor sols. The data presented in Table II indicate that the presence of $0.1 \mathrm{M}$ 
$\mathrm{NaNO}_{3}$ leads to sols with completely unacceptable properties. Although the addition of lesser amounts of $\mathrm{NaNO}_{3}$ gave sols with reascnable appearances, such sols could also be obtained without adding sodium nitrate. Thus, later preparations of boehmite sols did not include any sodium nitrate. (A separate study of the effect of ionic strength on the properties of gels prepared from boehmite sols was also performed. Details of this study are given in ref. 11.) The addition of phosphoric acid to the sols showed no deleterious effects on either the sols or the resulting xerogels.

\section{b. Effects of Sintering Conditions on the Properties of} Unsupported Membranes

\section{Effects of Sintering at $500^{\circ} \mathrm{C}$}

Further characterization of the monolithic xerogels described in the previous section was obtained by firing these xerogels to $500^{\circ} \mathrm{C}$ and determining their pore size distributions (PSDs) based on $\mathrm{N}_{2}$ adsorption-desorption isotherms. Figure 2 illustrates the sorption isotherms and PSDs for both the xerogel and the ceramic membrane produced from preparation $\mathrm{H} 4$. The gel, which was outgassed at $200^{\circ} \mathrm{C}$, showed primarily microporosity. Heating the xerogel to $500^{\circ} \mathrm{C}$ enlarged the pores to an approximate diameter of $4 \mathrm{~nm}$. This increase was unexpected because, although the material undergoes calcination, no real sintering or densification occurs at this tempexature. (A membrane prepared from $H 5$ was also analyzed by mercury porosimetry. The modal pore diameter was found to be about $4.5 \mathrm{~nm}$, a result which agrees well with our sorption isotherm results.) 
Sorption Isotherm

Gel

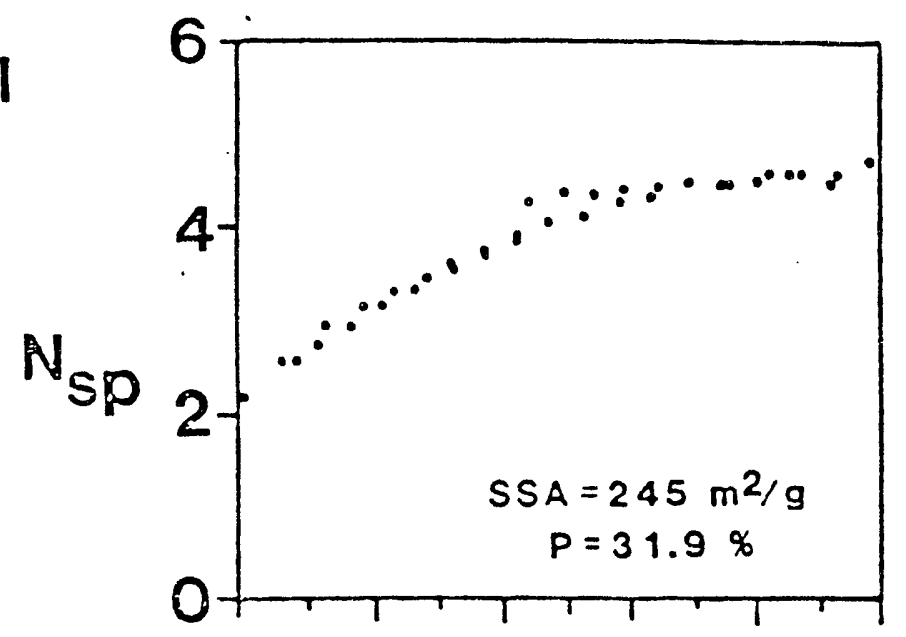

Ceramic

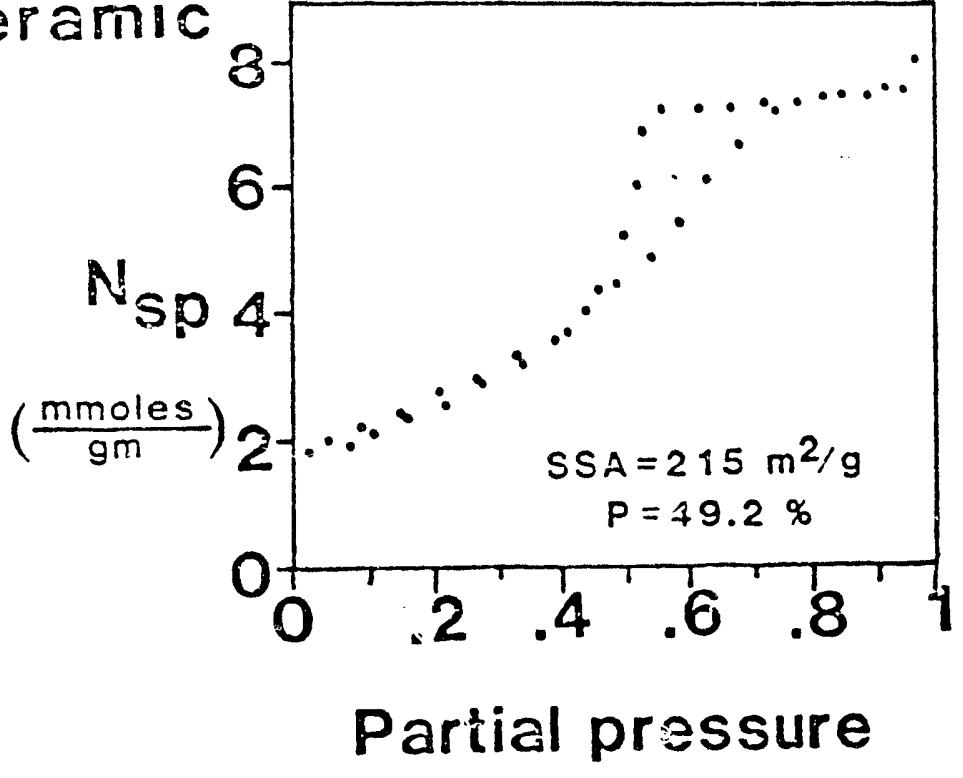

Pore Size Distribution
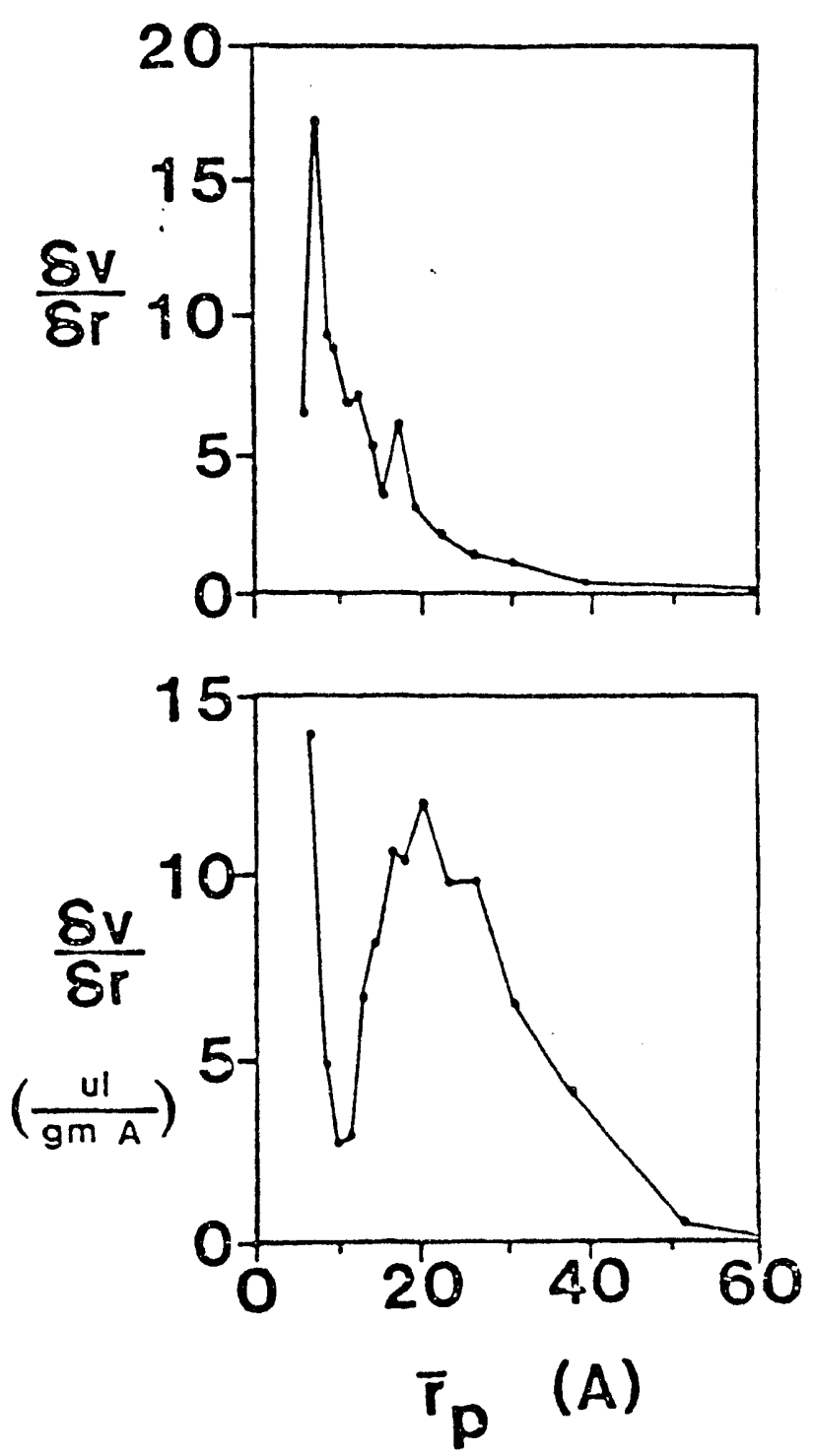

Figure 2. Nitrogen sorption isotherms and pore size distributions obtained from an alumina xerogel and ceramic membrane. Samples were prepared from hydrolys:s H4 (see Table II). P - porosity; SSA specific surface area. 
The PSDs shown in Figure 2 appear quite broad because they were calculated using the adsorption branch of the isotherm. Distributions determined from the desorption branch are always much narrower than those determined from the adsorption branch, so that PSDs from the desorption branch are usually reported. However, PSDs based on the desorption branch overemphasize the neck or narrow regions of the pore structure, while the adsorption branch better represents the PSD of the entire sample. Since solutes pass through the filter via the largest channels available, one needs to know if large pores are present. Thus, the results described in this section are based on PSD calculations performed using data for the adsorptiun branch of the isotherm. However, this policy has not been employed uniformly throughout this study. The data in Figure 3 illustrate the effect of the initial acid concentration on the resultant pore structure. The top PSD (sample H11) was obtained from the sample prepared with the least amount of acid that could be used to obtain a stable sol. This sample had very good total porosity (588), but the PSD was very broad. Moreover, the gel cracked while forming. When additional acid was present, membranes could be produced that remained intact during drying (Samples $\mathrm{H} 2, \mathrm{H} 4$ and $\mathrm{H} 5$ ). These membranes exhibited somewhat narrower PSDs yet had porosities of about 508 .

PSDs obtained for samples containing added $\mathrm{NaNO}_{3}$ or $\mathrm{H}_{3} \mathrm{PO}_{4}$ were generally very similar to that shown for Sample $\mathrm{H} 5$ in Figure 3. Except for Sample H7, the porosities of these membranes were all about 508. Sample H7 was characterized by a much broader PSD, and a total porosity of only 178 . On the basis of the appearance of the precursor sols and the PSDs obtained in this study, the formulation for Sample H10 was selected for use in preparing boehmite sols for further studies of supported membranes. The formulation for Sample H5 was 


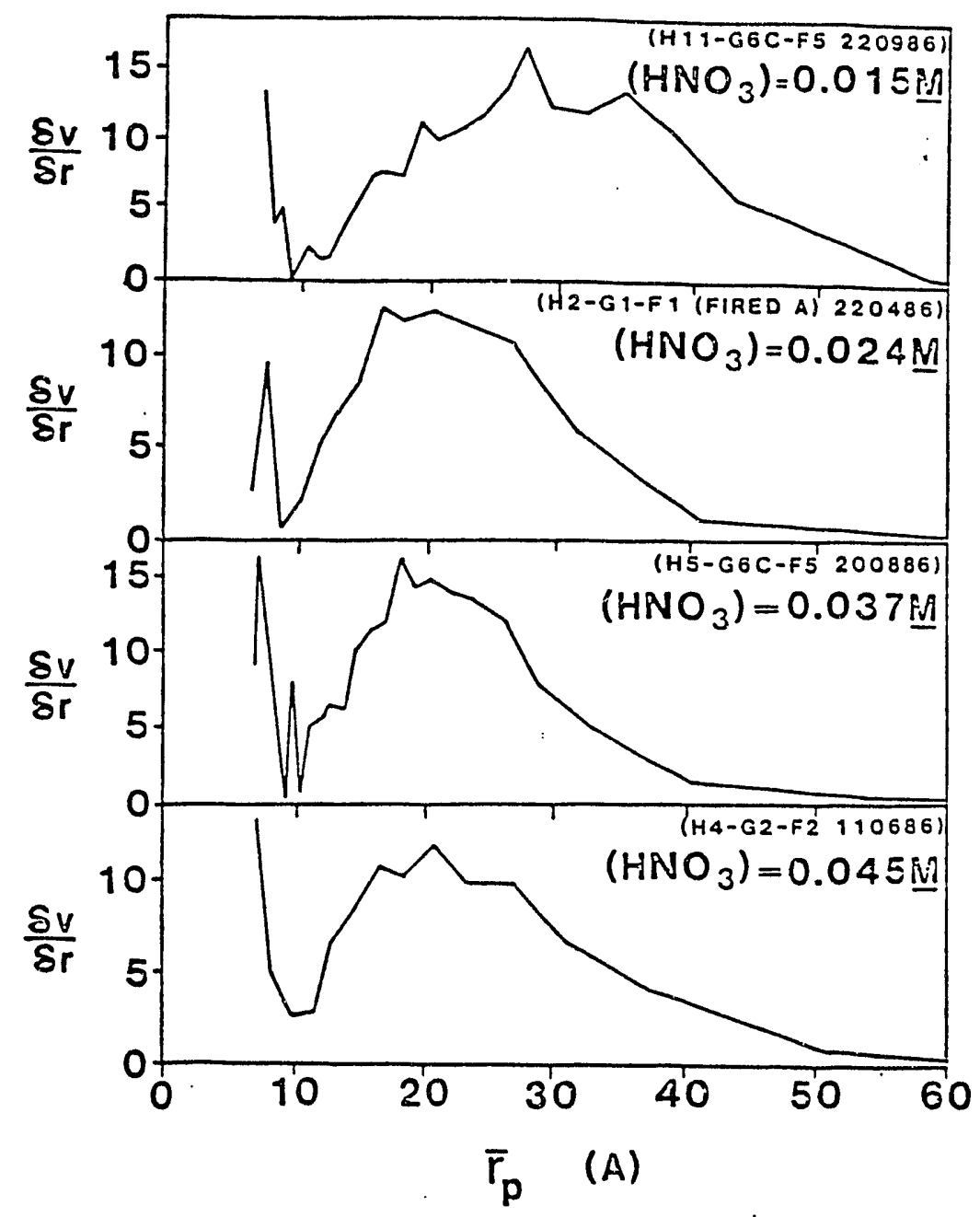

Figure 3. Effect of initial $\mathrm{HNO}_{3}$ concentration on the pore size distributions of ceramic membranes prepared from precursor sols H11, H2, H5 and H4.

also used on occasion. Supported membranes prepared using these two formulations displayed very similar properties, although a direct comparison of these membranes has not been performed [2].

It should also be noted that scanning electron photomicrographs of the fracture edges of these unsupported membranes showed no voids or defects in the bulk membrane and indicated a fairly constant particle size (ca. $60 \mathrm{~nm}$ ) and shape. This result agrees well with a separate determination of the particle size in the precursor sol using photon correlation spectroscopy. Although it is not possible to determine whether these particles are aggre- 
gates or primary particles, the agreement in the sizes measured using these techniques indicates that the particles imaged in SEM are the same ones present and measured in the suspension after sonication for $20 \mathrm{~min}$ [1].

\section{Effects of Varying the Sintering Temperature}

Ten boehmite xerogels were prepared from hydrolysis H5 (see Table II): $[\mathrm{Al}]_{\mathrm{T}}=0.5 \mathrm{M}, \mathrm{HNO}_{3} / \mathrm{Al}_{\mathrm{T}}=0.07$ mole ratio, and $\mathrm{pH}_{\text {final }}=3.9$. Nine of the gels were fired to different temperatures between 425 and $1600^{\circ} \mathrm{C}$. A phase transformation between boehmite and $\boldsymbol{\gamma}$-alumina occurs around $500^{\circ} \mathrm{C}$. Transition alumina phases persist up to $1100-1200^{\circ} \mathrm{C}$, where a major atomic rearrangement leading to $\alpha$-alumina occurs. This last change is accompanied by a significant increase in grain size. By the time the firing temperature reached $1100^{\circ} \mathrm{C}$, the samples had shrunk and gradually changed from clear to translucent to opaque white as a consequence of these events.

Results of $\mathrm{N}_{2}$ sorption measurements on these unsupported membranes are given in Figure 4, which shows the effect of sintering temperature on the surface area and pore structure of these samples. In general the surface area decreased with increasing sintering temperature. At sintering temperatures between 425 and $950^{\circ} \mathrm{C}$, the porosity remained fairly constant $(43-498)$, but it fell to 0 of at $1250^{\circ} \mathrm{C}$. Although this loss of porosity might be attributable to pore closure, this hypothesis is contradicted by the fact that the mean radius of the pores actually increased with increasing sintering $t$ mperature. SEM pictures of these samples showed that, at sintering temperatures $\leq 1100^{\circ} \mathrm{C}$, the microstructure of the membranes was composed of small spherical aggregates. At sintering temperatures above $1100^{\circ} \mathrm{C}$, significant grain growth occurred with 

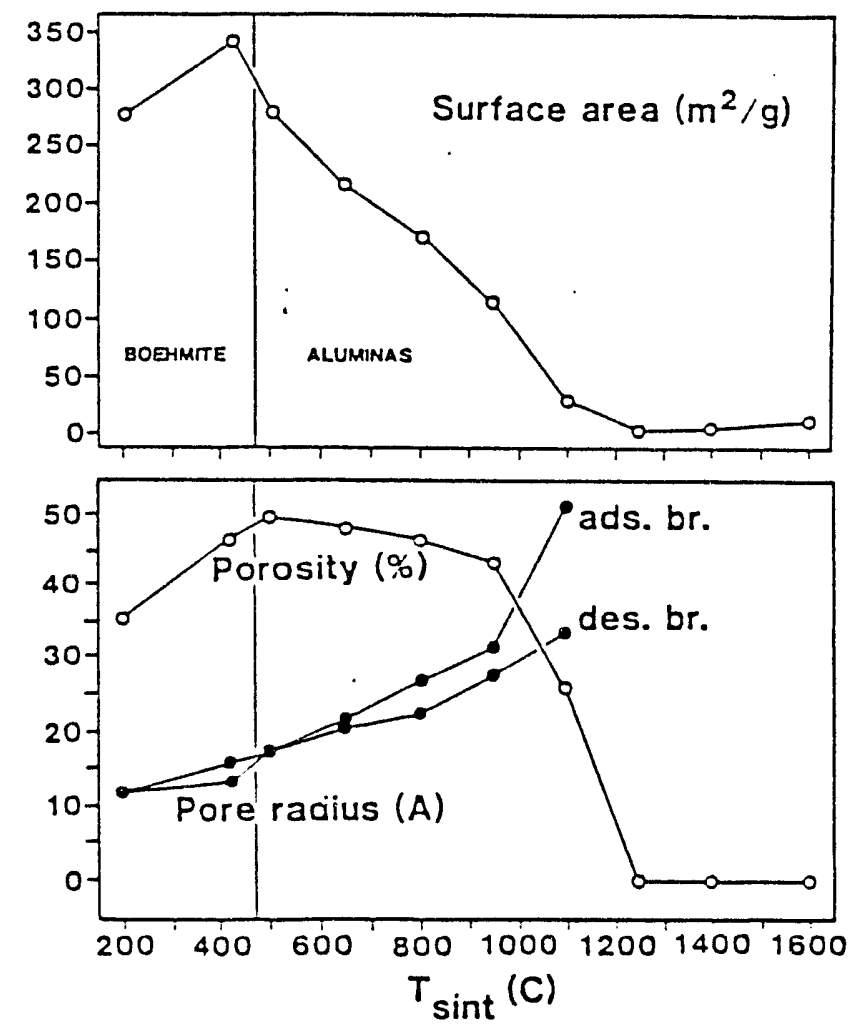

Figure 4. Effect of sintering temperature on the surface area and mean pore size of boehmite xerogels and alumina membranes. (The data for temperatures greater than $1100^{\circ} \mathrm{C}$ are misleading - see text.)

a concomitant increase in pore size. Although these samples retain some porosity, the pore sizes cannot be measured by $\mathrm{N}_{2}$ sorption since very little capillary condensation occurs in the large pores that form when alumina membranes are sintered at these temperatures.

c. Effects of Solid Concentration in the Sol on the Properties of Unsupported Membranes

In silica sols prepared by the Stöber process, when one increases the concentration of solid in the sol, both the particle size and the pore size increase. Therefore, an experiment was performed to see if the same phenomena 
occur in the boehmite/alumina system. Four sols with different solids concentrations were prepared while holding the $\mathrm{HNO}_{3} / \mathrm{Al}_{\mathrm{T}}$ mole ratio constant at 0.07 (see Table III). The sols were then either concentrated or diluted to solids concentrations of 2.2 wtz. Aliquots of these sols were gelled using the usual procedure, and the resultant membranes were fired to $500^{\circ} \mathrm{C}$. Results of $\mathrm{N}_{2}$ sorption measurements on these samples are listed in Table III. Inspection of these results indicates that there is no obvious effect of the solid concentration in the precursor sol on either the surface area or pore radius of $\gamma$ alumina membranes.

Table III. Effect of solid concentration in the precursor sol on the pore structure of unsupported membranes of $\gamma$-alumina.

\begin{tabular}{ccccc} 
Sample & $\begin{array}{c}\text { Solids Content of } \\
\text { original sol } \\
(\text { wtz) }\end{array}$ & $\begin{array}{c}\text { Properties of Membrane Fired at } 500^{\circ} \mathrm{C} \\
\text { Porosity } \\
(8)\end{array}$ & $\begin{array}{c}\text { Surface area } \\
\left(\mathrm{m}^{2} / \mathrm{g}\right)\end{array}$ & $\begin{array}{c}\text { Pore radius } \\
(\AA)\end{array}$ \\
\hline & & & 303 & 17.5 \\
A & 0.55 & 51.0 & 285 & 17.8 \\
B & 1.1 & 47.8 & 306 & 16.8 \\
C & 2.2 & 49.7 & 312 & 17.5 \\
D & 4.4 & 51.7 & &
\end{tabular}

d. Effects of Phosphate Addition on the Properties of Unsupported Membranes

In earlier sections the effects of adding phosphate on the properties of the resulting unsupported membranes were shown to be quite small. However, further experiments were performed to better define the possible effects of incorporating phosphate in the synthesis of these membranes, since phosphate could be added at several different stages of the synthesis procedure. 
Two forms of sol instability are recognized - typical aggregation and coacervation. Aggregation produces either solid precipitates or cloudy, inhomogeneous, partially gelled sols. Coacervation, which is uncommon, is defined as the formation of a liquid precipitate by coagulation of a sol. Since the interparticle bonds in coacervates are initially labile, coacervates are initially fluid. However, they may gel if left undisturbed. (Another description of coacervation is the spontaneous separation of a sol into colloid-rich and colloid-poor regions which may then gel independently of one another.) Both types of sol instability were observed as a result of phosphate addition. Coacervates tended to form when comparatively high phosphate concentrations $(>0.02 \mathrm{M})$ were used.

Three variables were studied: phosphate concentration, the form in which phosphate was added, and the point in the hydrolysis at which phosphate was added.

1. Phosphate concentrations were varied from $10^{-5} \mathrm{M}$ to $0.1 \mathrm{M}$.

2. Two forms of phosphate were added in separate experimenis: $\mathrm{KH}_{2} \mathrm{PO}_{4}$ (or $\mathrm{NaH}_{2} \mathrm{PO}_{4}$ ) and $\mathrm{H}_{3} \mathrm{PO}_{4}$. Addition of the $\mathrm{H}_{2} \mathrm{PO}_{4}^{-}$salts should result in chemical interactions between $\mathrm{H}_{2} \mathrm{PO}_{4}{ }^{-}$and boehmite. Addition of these salts should both increase the ionic strength of the solution and have a secondary effect on solution $\mathrm{pH}$. Similar changes in the system should occur on addition of phosphoric acid. However, addition of $\mathrm{H}_{3} \mathrm{PO}_{4}$ should lower the system $\mathrm{pH}$ much more significantly than should addition of dihydrogen phosphate salts.

3. Phosphate can be added to the system at three stages prior to gel formation. These stages have been designated "early", "late", and "cold". "Early" refers to the addition of phosphate to the water used 
for initial hydrolysis of the aluminum alkoxide. At this time, only phosphoric acid was added so as to prevent inclusion of sodium or potassium ions in the boehmite crystallites. The experiments described in conjunction with the discussion of Table II were performed at this stage. "Late" refers to addition of phosphate after peptization of the boehmite suspension using $\mathrm{HNO}_{3}$. Both "early" and "late" additions require overnight refluxing of the phosphate-boehmite mixture at $100^{\circ} \mathrm{C}$. "Cold" refers to addition of phosphate to the sol after the boehmite is peptized, refluxed overnight and cooled to room temperature. Hence, during the "cold" addition experiments, the mixture of phosphate and boehmite was never heated subsequent to the addition of phosphate. "Early" addition of up to $0.001 \mathrm{M}$ phosphoric acid had no effect on sol appearance, gel cracking, or the PSDs of membranes fired at $500^{\circ} \mathrm{C}$. These results are essentially the same as those obtained for membranes to which no phosphate had been added. "Early" addition of $0.01 \mathrm{M}$ phosphoric acid inhibited peptization, i.e., a precipitate remained in the sol. The xerogel prepared from this unstable sol cracked while drying. "Late" addition of $0.01 \mathrm{M}$ phosphoric acid did not cause the already peptized sol to precipitate, but "late" addition of $0.05 \mathrm{M}$ phosphoric acid led to formation of a coacervate. Xerogels formed from these two sols spontaneously cracked while drying.

"Cold" additions of $\mathrm{KH}_{2} \mathrm{PO}_{4}$ and $\mathrm{H}_{3} \mathrm{PO}_{4}$ at concentrations up to $0.01 \mathrm{M}$ did not affect the stability of the sol. Thin, uncracked xerogels formed under these conditions. Addition of $0.1 \mathrm{M}$ phosphate to "cold" systems destabilized the sols; and, although the xerogels remained whole, they shrank considerably. Because of this shrinkage and the thick, chunky nature of the resulting 
xerogels, this formulation would not be expected to be useful for fabricating membranes.

Unfortunately, the only nitrogen adsorption measurements available for these systems are those for the fired unsupported membranes from hydrolyses $\mathrm{H} 9$ and $\mathrm{H} 10$ ("early" additions of $10^{-5} \mathrm{M}$ and $0.001 \mathrm{M} \mathrm{H}_{3} \mathrm{PO}_{4}$ ). As noted above, the pore size distributions for these unsupported membranes were identical to those of the corresponding phosphate-free membranes. In general, it appeared that heating the phosphate-sol mixtures destabilized the sol. Only very low concentrations of phosphate could be tolerated before deleterious effects were seen in both the sols and the xerogels.

If the membrane porosity occurs primarily within the particles and if the phosphate is to have any effect on the pore structure, then the addition of phosphate must be made before the hydrolysis step. However, "early" addition of up to $0.001 \mathrm{M}$ phosphate had little effect on the pore structure while addition of $0.01 \mathrm{M}$ phosphate inhibited peptization. Consequently, it does not appear likely that phosphate affects the nature of the intra-particle porosity. A separate study in which phosphate was incorporated into supported alumina membranes (see section VI.B.3) also indicates that phosphate has little effect on the permselectivities of these ceramic membranes.

\section{e. Summary}

Unsupported alumina membranes with pore diameters of ca. $40 \dot{A}$ were fabricated from boehmite sols that were prepared by the hydrolysis and peptization of aluminum tri-sec-butoxide. Monolithic (uncracked) membranes could be prepared only when the precursor suspensions were stable. Typical pH 
values for these sols were between 3.5 and 4.0. If the $\mathrm{pH}$ of the sol was much lower than 3.5 , the boehmite would dissolve, while boehmite particles in sols with $\mathrm{pH}$ values above 4.0 tended to aggregate.

The primary factor that controlled the pore structures (i.e., pore sizes, porosities and specific surface areas) of these unsupported membranes was the temperature at which the boehmite xerogels were fired. When these xerogels were fired to $500^{\circ} \mathrm{C}$, at which temperature the boehmite transformed to $\boldsymbol{\gamma}$-alumina, the membranes with the highest porosities (ca. 498) were obtained. These membranes had corresponding specific surface areas above $250 \mathrm{~m}^{2} / \mathrm{g}$ and pore diameters of ca. $36 \AA$.

Since $\gamma-\mathrm{Al}_{2} \mathrm{O}_{3}$ is less soluble in water than boehmite, it is recommended that these membranes be fired to at least $500^{\circ} \mathrm{C}$. However, when these membranes were fired at temperatures above $500^{\circ} \mathrm{C}$, the pore diameters of these membranes were larger than the pore diameters of similar membranes that were fired at $500^{\circ} \mathrm{C}$. Thus, the preferred firing temperature for these unsupported alumina membranes is $500^{\circ} \mathrm{C}$. This temperature was also chosen as the firing temperature for the supported alumina membranes used for permeability and permselectivity studies. However, there is some evidence which indicates that the properties of supported membranes are affected by interactions between the support and the material comprising the membrane. Further studies of the effects of sush interactions are warranted.

The solids concentration in the precursor sol was found to have little effect on the pore structures of unsupported alumina membranes fired at $500^{\circ} \mathrm{C}$, at least for solids concentrations between 0.55 and 4.4 wt 8 . Addition of phosphate to the precursor sol either had no effect on the pore structures of resulting membranes or else caused the xerogels to crack while drying. (A 
possible exception to these observations could have occurred in systems prepared by the "cold" addition to boehmite sols of $\mathrm{KH}_{2} \mathrm{PO}_{4}$ and $\mathrm{H}_{3} \mathrm{PO}_{4}$ at concentrations up to $0.01 \mathrm{M}$. While monolithic xerogels could be formed from these sols, the pore structures of membranes prepared from these xerogels were not determined. Thus, the effect of phosphate addition in these systems is not known.) When small amounts of phosphate $(<0.001 \mathrm{M})$ were added to the other systems, apparently there was not enough phosphate present to noticeably affect the pore structures of the resulting membranes. When larger amounts of phosphate were added, both the $\mathrm{pH}$ and ionic strength of the precursor sol were affected, and deleterious changes in the sol often occurred (e.g., formation of precipitates and coacervates).

It appears that useful alumina membranes can be formed only from sols with $\mathrm{pH}$ values and ionic strengths that fall within a fairly narrow range. While changes in the solids concentration have relatively little effect on the sol $\mathrm{pH}$ and ionic strength, changes in the concentration of phosphate added to the sol have a much more dramatic effect on these parameters. It is still possible that conditions exist for which the addition of phosphate to boehmite sols will improve the properties of alumina membranes fabricated from those sols. However, it appears that such conditions would involve only a limited range of phosphate concentrations and, in addition, would depend on the $\mathrm{pH}$, ionic strength and solids concentration of the particular sol under investigation. Thus, the determination of those conditions will not be an easy problem to solve. 
2. Silica

Because of the relatively high solubility of alumina at low pH values, we wanted to prepare membranes from materials that would be more stable under such conditions. One such material is silica. In addition, once silica membranes are prepared and characterized, one can then attempt to synthesize mixed aluminosilicate materials. While no mixed $\mathrm{SiO}_{2} / \mathrm{Al}_{2} \mathrm{O}_{3}$ membranes were fabricated during this project, the preparation and characterization of such materials is a long-term goal of our research program. It should also be noted that more recent attempts to replicate the syntheses of some of the membranes described below, particularly those that produced crack-free structures, did not succeed in producing crack-free membranes. Since no other properties of these replicate membranes were measured, it is not known how closely these properties match those of the membranes described below.

\section{a. Experimental Method}

Suspensions of silica particles were obtained by the hydrolysis of tetraethyl orthosilicate (TEOS) in ammonium hydroxide $\left(\mathrm{NH}_{4} \mathrm{OH}\right)$. Unsupported silica membranes were prepared by slow evaporation at room temperature. Four major parameters were examined to determine their effects on particle and membrane properties:

1. Choice of solvent.

2. Silica/ammonia $\left(\mathrm{TEOS} / \mathrm{NH}_{4} \mathrm{OH}\right)$ mole ratio during hydrolysis.

3. Solids concentration in solution.

4. Sintering temperature. 
b. Effects of the Solvent Employed on the Properties of

Unsupported Membranes

Sols did not appear to differ visually regardless of whether sthanol, water or sonicated water was used as the solvent and gelling medium [2]. The data obtained from nitrogen sorption determinations supported the conclusion that the type of solvent employed had little effect on either the porosity or the PSD of unsupported membranes. Table IV reflects the resulting sirularities. However, crack-free xerogels were obtained only from aqueous sols.

Table IV. Effects of gelling solvent and silica/ammonia mole ratio on the pore structure of silica membranes.

\begin{tabular}{lccccc}
$\begin{array}{c}\text { Gelling } \\
\text { Solvent }\end{array}$ & $\begin{array}{c}\mathrm{Si} / \mathrm{NH}_{3} \\
\text { mole ratio }\end{array}$ & $\begin{array}{c}\text { o Solid } \\
(\text { wt } / \text { vol })\end{array}$ & $\begin{array}{c}\text { Surface Area } \\
\left(\mathrm{m}^{2} / \mathrm{g}\right)\end{array}$ & $\begin{array}{c}\text { Porosity } \\
(8)\end{array}$ & $\begin{array}{c}\text { Pore Radius } \\
(\AA)\end{array}$ \\
\hline \multirow{2}{*}{ Ethanol } & 1.51 & 1.96 & 758 & 68.3 & 31 \\
& 1.01 & 1.95 & 557 & 67.3 & 48 \\
& 0.76 & 1.93 & 379 & 61.5 & 68 \\
& 0.61 & 1.92 & 305 & 62.0 & 114 \\
& 0.61 & 1.92 & 231 & 44.6 & 57 \\
& 0.50 & 1.91 & 231 & 51.6 & 88 \\
& 0.43 & 1.89 & 188 & 46.9 & 89 \\
Water & 0.38 & 1.88 & 62 & 40.5 & 90 \\
& & & & & \\
& 1.51 & 1.96 & 528 & 67.4 & 35 \\
& 1.01 & 1.95 & 440 & 66.9 & 51 \\
& 0.76 & 1.93 & 313 & 57.3 & 81 \\
& 0.61 & 1.92 & 271 & 56.7 & 93 \\
& 0.50 & 1.91 & 257 & 50.6 & 90 \\
Sonicated & 0.43 & 1.89 & 247 & 48.2 & 94 \\
Water & 0.38 & 1.88 & 220 & 44.4 & 95 \\
& 1.51 & 1.96 & 577 & 67.1 & 33 \\
& 1.01 & 1.95 & 438 & 68.1 & 50 \\
& 0.76 & 1.93 & 269 & 59.3 & 75 \\
& 0.61 & 1.92 & 266 & 53.7 & 94 \\
& 0.50 & 1.91 & 128 & 34.5 & 94 \\
& 0.43 & 1.89 & 246 & 48.6 & 100 \\
& 0.38 & 1.88 & 229 & 46.0 & 100
\end{tabular}


Note that th: idsorption branch of the BET isotherms gave pore size distributions that were too broad to provide a reasonable indication of the true pore size and PSD. Thus, the mean pore radii reported for silica membranes are based on the desorption branch of the BET isotherm.

c. Effects of Reactant Concentrations on the Properties of Unsupported Membr:ses

The TEOS $/ \mathrm{NH}_{4} \mathrm{OH}$ mole ratio was decreased by increasing the concentration of $\mathrm{NH}_{4} \mathrm{OH}$ while holding the solids concentration rear $2 \%$. Decreases in this ratio produced progressively cloudier sols and xerogels. The sols themselves were less stable at lower $\mathrm{TEOS} / \mathrm{NH}_{4} \mathrm{OH}$ mole ratios [2]. Inspection of the data in Table IV and Figure 5 indicates that the mean pore size in the membranes

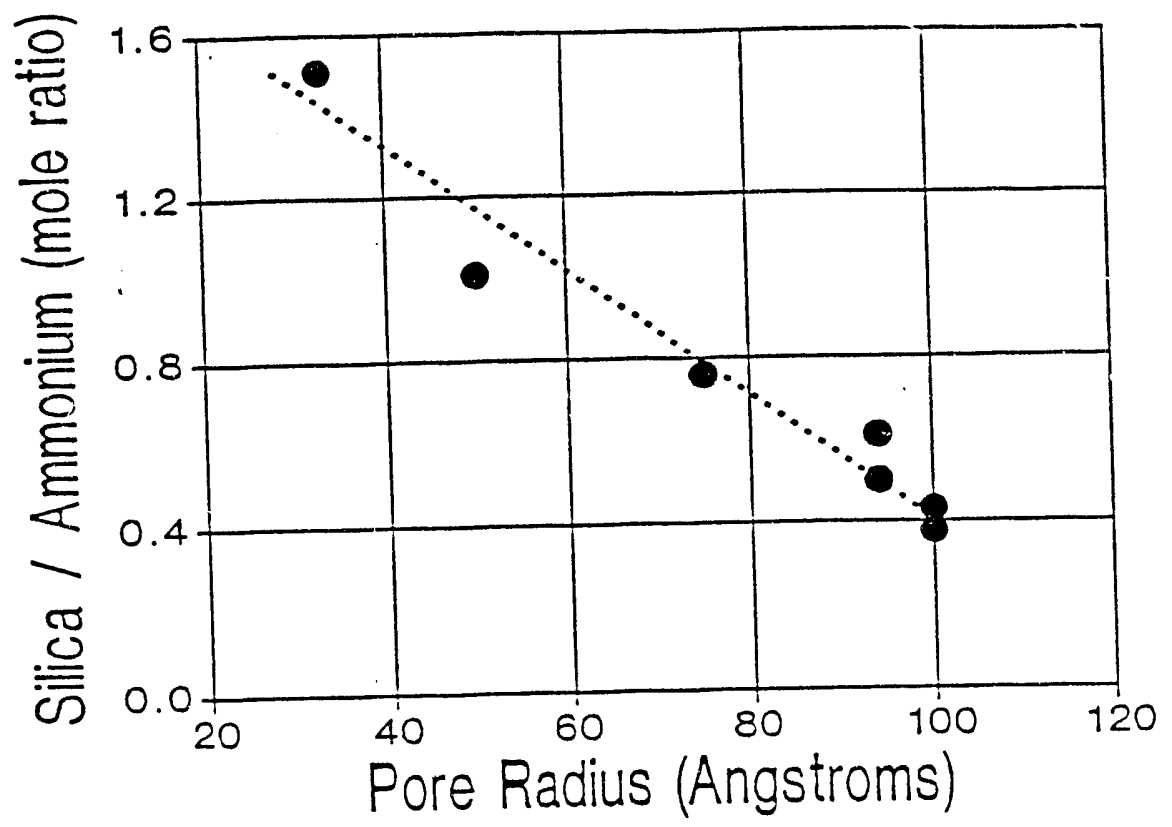

Figure 5. Effect of the silica/ammonia mole ratio on the mean pore size for membranes prepared from sols in which sonicated water is the solvent. 
decreased as the silica/ammonia mole ratio increased, irrespective of the type of solvent employed. Two membranes were prepared from ethanol based sols with a $\mathrm{Si} / \mathrm{NH}_{3}$ mole ratio of 0.61 because the properties of the first membrane did not appear to be consistent with the other results. The replicate sample was characterized by properties that fit the general pattern described above.

\section{d. Effects of Solids Concentration on the Properties of} Unsupported Membranes

When the solids concentration is increased in sols prepared by the Stöber process, both the size of the precursor particles and the pore size increase. This effect was investigated by inzreasing the percentage of silica present in ethanolic sols while holding the $\mathrm{Si} / \mathrm{NH}_{3}$ mole ratio constant at 1.21. Visual examination indicated the formation of progressively cloudier sols and xerogels as the silica concentration increased. Inspection of the data in Table $\mathrm{V}$ and Figure 6 reveals that the mean pore radius was linear in the solids concentration. Since our objective was to create membranes with small pore sizes, two further experiments were conducted.

1. Sols \#31 and \#28 were concentrated (by factors of 3.33 and 7 , respectively) via rotary evaporation prior to the fabrication of membranes. Later, these sols were resuspended by diluting them to their original volume with ethanol and sonicating. This treatment produced sols whose s lids concentrations simulated those of the original sols. Inspection of the data in Table $V$ shows that the diluted sols (\#40 and \#39) yielded membranes whose mean pore sizes were very similar to those of the membranes formed from their more concentrated counterparts. 
Table V. Effects of solids concentration in the precursor ethanolic sol on the pore structure of the resultant silica membranes $\left(\mathrm{Si} / \mathrm{NH}_{3}=\right.$ 1.21).

\begin{tabular}{cccccc}
$\#$ & $\begin{array}{c}\text { of Solids } \\
\text { (wt/vol) }\end{array}$ & $\begin{array}{c}\text { o Solids } \\
(\text { wt } / \text { vol) }\end{array}$ & $\begin{array}{c}\text { Surface Area } \\
\left(\mathrm{m}^{2} / \mathrm{g}\right)\end{array}$ & $\begin{array}{c}\text { Porosity } \\
(8)\end{array}$ & $\begin{array}{c}\text { Pore Radius } \\
(\dot{A})\end{array}$ \\
\hline 31 & 0.25 & 1.67 & 739 & 57.5 & 18 \\
28 & 0.52 & 1.74 & 675 & 66.2 & 28 \\
29 & 1.95 & 1.95 & 541 & 66.8 & 42 \\
30 & 6.24 & 6.24 & 227 & 35.9 & 10 \\
35 & 0.24 & 0.48 & 775 & 57.2 & 11 \\
37 & 0.52 & 1.05 & 710 & 59.6 & 18 \\
40 & 1.67 & 0.24 & 707 & 62.4 & 19 \\
39 & 1.74 & 0.52 & 647 & 66.9 & 34
\end{tabular}

a of Solids present in the original suspension.

b \& Solids present in the suspension at the start of gelation.

Sample \# Unsupported Membrane Preparation

31 concentrated to $1 / 3$ the original sol volume

28 concentrated to $1 / 7$ the original sol volume

$38 \& 37$ concentrated to $1 / 2$ the original sol volume

40 diluted with EtOH by a factor of 3.33 to simulate the original sol 31

39 diluted with EtOH by a factor of 7.00 to simulate the original sol 28

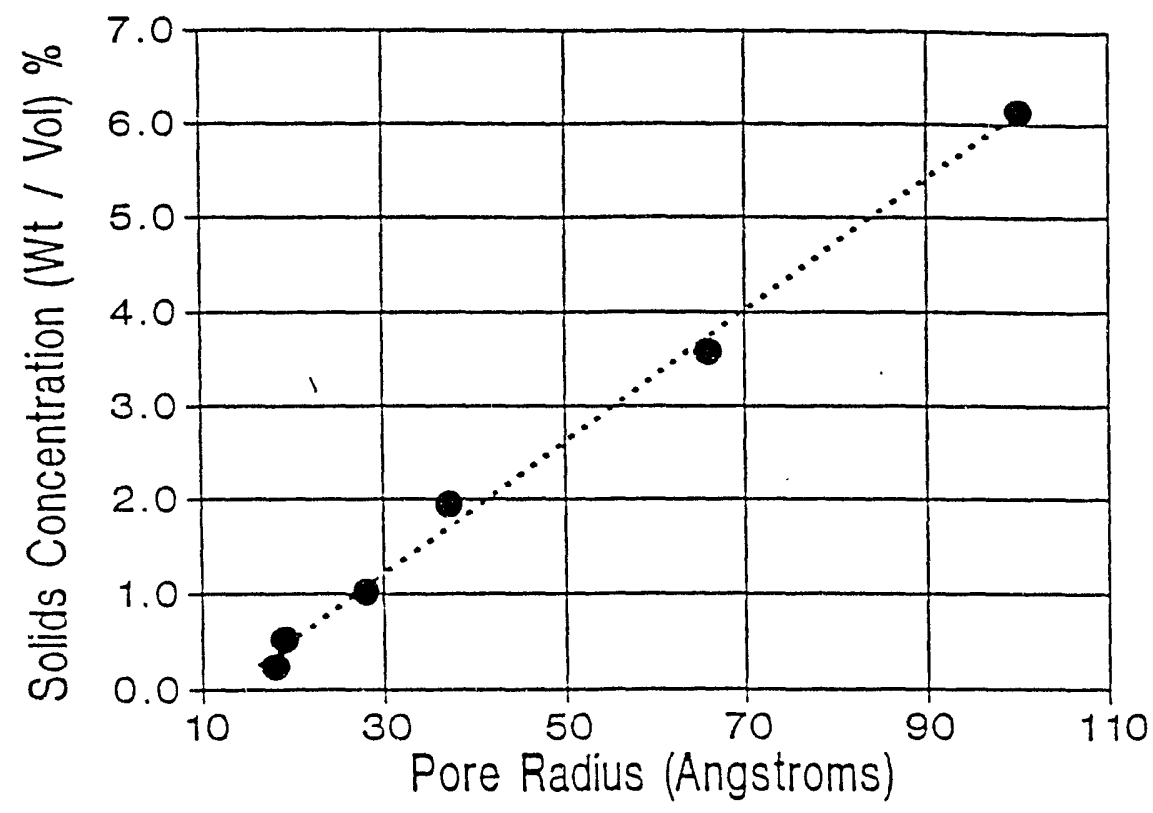

Figure 6. Effect of solids concentration on the mean pore radius of unsupported silica membranes prepared from ethanolic sols. 
2. We next reproduced sols $\# 31$ and \#28. This time however, we concentrated these sols by a factor of two to obtain sols \#38 and \#37. These sols were used to produce membranes that had significantly smaller mean pore radii than any other membranes that had been produced up to this point.

Scanning electron microscopy was employed to estimate the range of particle sizes. The data in Table VI indicate that as the solids concentrations were increased from 0.528 (sample \#19) to 6.148 (sample \#23) the corresponding mean pore diameters increased from ca. $40 \AA$ to ca. $200 \AA$. At this time, it appeared that the best protocol for preparing crack-free silica xerogels would involve the use of dilute aqueous silica sols. Such sols would contain only enough concentrated $\mathrm{NH}_{4} \mathrm{OH}$ to provide the stoichiometric amount of water necessary for complete hydrolysis of TEOS under basic conditions. When sample \#31 was prepared following such a protocol, we were also able to confirm our hypothesis that more dilute sols give membranes composed of smaller particles.

Table VI. Effects of solids concentration on the size of the precursor silica particles and on the mean pore radius of silica membranes.

\begin{tabular}{|c|c|c|c|c|c|}
\hline \# & $\begin{array}{l}\text { o Solids } \\
\text { (wt/vol) }\end{array}$ & $\begin{array}{c}\text { Particle Size } \\
\text { Range }(\mathrm{nm})\end{array}$ & $\begin{array}{c}\text { Surface Area } \\
\left(\mathrm{m}^{2} / \mathrm{g}\right)\end{array}$ & $\begin{array}{c}\text { Porosity } \\
(8)\end{array}$ & $\begin{array}{c}\text { Pore Radius } \\
(\dot{A}) \\
\end{array}$ \\
\hline 31 & 0.24 & $<30-$ & 739 & 57.5 & 18 \\
\hline 19 & 0.52 & $35-$ & 681 & 63.5 & 19 \\
\hline 20 & 1.02 & $50-85$ & 1296 & 78.1 & 28 \\
\hline 21 & 1.95 & $35-85$ & 483 & 64.8 & 37 \\
\hline 22 & 3.58 & $100-135$ & 257 & 53.8 & 66 \\
\hline 23 & 6.14 & $135-200$ & 208 & 45.8 & 100 \\
\hline
\end{tabular}


e. Effects of Sintering Temperature on the Properties of

Unsupported Membranes

Four identical samples were fired at four different temperatures. The data in Table VII and Figure 7 demonstrate that the mean pore radius increased from $42 \AA$ to $58 \AA$ when the firing temperature was raised from 200 to $400^{\circ} \mathrm{C}$. When the samples were fired at $1000^{\circ} \mathrm{C}$, however, the mean pore radius decreased markedly to $29 \dot{A}$. This decrease was accompanied by a loss of more than half the porosity of the sample. This inconsistent behavior has not yet been satisfactorily explained.

Table VII. Effects of the sintering temperature on the pore structure of silica membranes.

\begin{tabular}{cccc}
$\begin{array}{c}\text { Sintering } \\
\text { Temperature } \\
\left({ }^{\circ} \mathrm{C}\right)\end{array}$ & $\begin{array}{c}\text { Surface } \\
\text { Area } \\
\left(\mathrm{m}^{2} / \mathrm{g}\right)\end{array}$ & $\begin{array}{c}\text { Porosity } \\
(8)\end{array}$ & $\begin{array}{c}\text { Pore } \\
\text { Radius } \\
(\dot{\mathrm{A}})\end{array}$ \\
\hline 200 & 541 & 66.8 & 42 \\
400 & 664 & 69.7 & 58 \\
600 & 476 & 66.8 & 54 \\
800 & 310 & 62.6 & 52 \\
1000 & 82 & 28.9 & 29
\end{tabular}

f. Summary

Precursor silica sols were synthesized by hydrolyzing tetraethyl orthosilicate with ammonium hydroxide using water, sonicated water and ethanol as possible solvents. For any particular concentration of reactants, the pore structures (pore diameters, porosities and specific surface areas) of the 


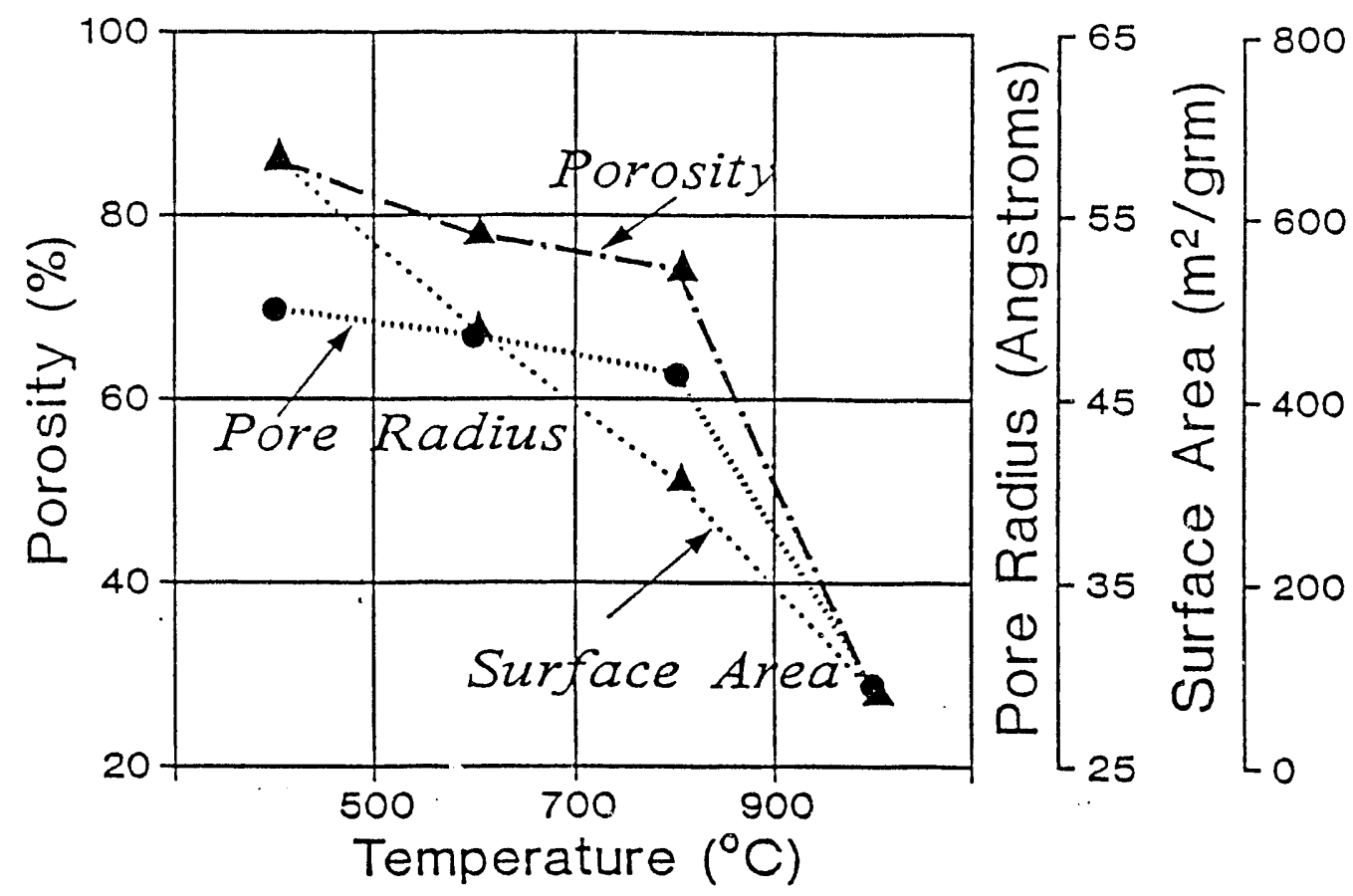

Figure 7. Effects of sintering temperature on the properties of unsupported silica membranes.

resulting silica membranes were independent of the solvent employed in fabricating the membranes. However, crack-free xerogels were obtained only from aqueous silica sols. (Recent attempts to fabricate these crack-free xerogels from aqueous sols have not been successful.)

The pore structures of these silica membranes depended strongly on the concentrations of the reactants employed in the hydrolysis. When a silica: ammonia mole ratio of 1.5 was utilized, the resultant membranes were characterized by extremely high specific surface areas $\left(>500 \mathrm{~m}^{2} / \mathrm{g}\right)$ and porosities (ca. 68z), as well as fairly large pore diameters (between 60 and $70 \dot{A}$ ). When the silica:ammonia mole ratio was decreased to 0.5 , the resultant membranes were characterized by smaller specific surface areas (between 120 and 260 $\mathrm{m}^{2} / \mathrm{g}$ ) and porosities (ca. 508). However, the pore diameters of these latter 
membranes increased markedly (to between 170 and $190 \AA$ ) and the silica sols from which these membranes were fabricated were less stable when a smaller mole ratio of silica:ammonia was used to prepare these materials.

The pore structures of these silica membranes were also dependent on the solids concentration of the precursor silica sols. (This observation was not true of alumina systems.) Utilizing a relatively dilute suspension of silica in ethanol ( 0.248 solids by $w t / v o 1)$ and a silica:ammonia mole ratio of 1.21 , we fabricated a silica membrane characterized by a mean pore diameter of ca. $20 \dot{A}$ and a porosity of $57 \%$. In general, it appears that more dilute sols give membranes composed of smaller particles.

Only a limited study of the thermal stability of these membranes was performed. Their porosities and pore diameters were found to decrease relatively slowly with increasing firing temperature, up to $800^{\circ} \mathrm{C}$. However, when the firing temperature was then increased to $1000^{\circ} \mathrm{C}$, both the porosity and the pore dianeter decreased markedly. The specific surface areas of these membranes exhibited a different behavior with respect to the firing temperature. The specific surface areas decreased nearly linearly as the firing temperature was increased from 400 to $1000^{\circ} \mathrm{C}$.

The production of silica membranes with very high specific surface areas (several hundred $\mathrm{m}^{2} / \mathrm{g}$ ) and relatively large mean pore diameters $(>60 \dot{A}$ ) was an unexpected result that has not yet been satisfactorily explained. One could speculate that the membranes have bimodal pore size distributions (PSDs) with one component consisting of a large volume of micropores. Since these PSDs are determined by analyzing the $\mathrm{N}_{2}$ sorption data using the Kelvin equation, which is not valid for pores with diameters less than ca. $16 \dot{\AA}$, there is a lower diameter cutoff for these PSDs. If most of the pores in these membranes 
have diameters of ca. $10 \AA$, such pores would not be observed in the PSDs but would be responsible for the very large specific surface areas that were measured for these membranes. At this time, however, it was concluded that further studies of silica membranes prepared in this manner would not be pursued because of the relatively large pore diameters that had been obtained. 


\section{Titania}

Considerable effort has been devoted to preparing and characterizing titania ceramic membranes. Crystalline titania, either as anatase or rutile, is insoluble except under strongly acidic conditions. Thus, titania membranes could be used for separations under conditions that would cause alumina membranes to dissolve. In addition, titania (especially in the anatase form) has very useful photocatalytic properties that may eventually lead to commercial applications of anatase membranes for environmental remediation.

Our initial studies resulted in the development of protocols for preparing both particulate and polymeric sols of titania. Further characterization of these materials has focused almost entirely on the particulate sols because we expect to obtain better control over the final structure of the membrane by using particulate sols. Therefore, information about preparing polymeric titania sols will be presented only in Section IV.A.3.a, and the remaining material in Section IV.A.3 will concern particulate titania sols. Figure 8 shows the general protocol employed to prepare ceramic membranes from both particulate and polymeric titania sols.

a. Experimental Method - Polymeric Sols

Polymeric titania sols were prepared by hydrolyzing titanium tetra-isopropoxide with a small quantity of water (some of which comes from conc. $\mathrm{HNO}_{3}$ ) dissolved in either ethanol or isopropanol (see Figure 8). Nitric acid (not shown in Figure 8) was added to the system to prevent the precipitation of titania particles. The small amount of water present in the system is not 


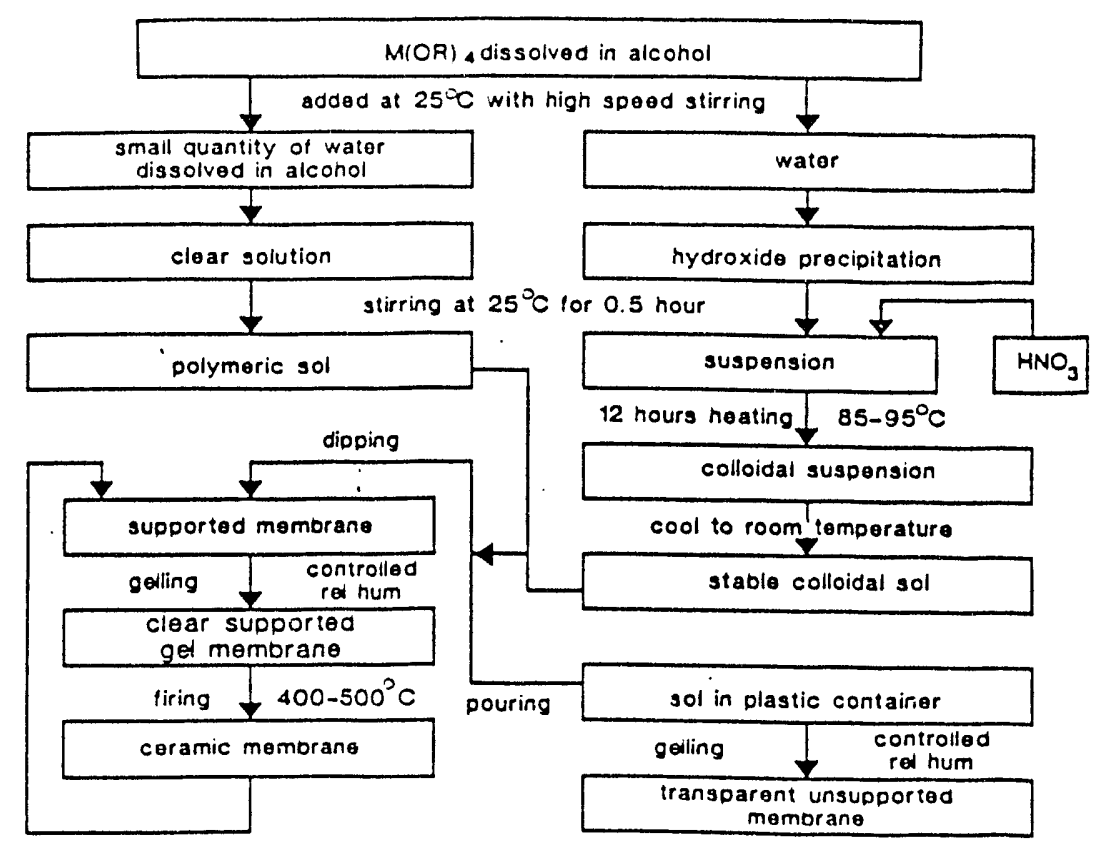

Figure 8. Procedures for preparing polymeric and particulate titania sols, as well as unsupported and supported titania membranes.

sufficient to allow complete hydrolysis of the metal alkoxide. The partially hydrolyzed products of the reaction, which are soluble in the alcoholic medium, condense by forming oxygen bridges and releasing water and isopropanol in a ratio which depends on the amount of water present in the initial reaction mixture. These condensation reactions eventually lead to the formation of polymeric chains. Gels were then produced by careful evaporation of the alcoholic suspensions.

The data in Table VIII illustrate the effects of changing the concentrations of various reactants on the properties of the resulting polymeric titania gels. The initial concentration of water controlled the viscosity of the so1: the more water, the more viscous the solution. Water also affected the sol-gel transformation, as transparent gels could only be obtained when the molar ratio of water to titanium was 4 or less. 
Table VIII. Influence of reactant concentrations on the appearance of polyneric titania gels.

\begin{tabular}{|c|c|c|c|c|}
\hline $\begin{array}{c}\mathrm{H}_{2} \mathrm{O} / \mathrm{Ti} \\
\text { (mole ratio) }\end{array}$ & $\begin{array}{c}\mathrm{H}^{+} / \mathrm{Ti} \\
\text { (mole ratio) }\end{array}$ & $\begin{array}{r}\mathrm{TiO}_{2} \\
\left(\begin{array}{ll}\mathrm{wt} & 8\end{array}\right) \\
\end{array}$ & $\begin{array}{c}\mathrm{EtOH} / \mathrm{Ti} \\
\text { (mole ratio) }\end{array}$ & $\begin{array}{c}\text { Appearance } \\
\text { of } \mathrm{Gel}\end{array}$ \\
\hline 1 & 0.08 & 3 & 50 & clear \\
\hline 2 & 0.08 & 3 & 50 & clear \\
\hline 4 & 0.08 & 3 & 50 & clear \\
\hline 8 & 0.08 & 3 & 50 & opaque \\
\hline 16 & 0.08 & 3 & 50 & $\begin{array}{l}\text { too viscous } \\
\text { to form gel }\end{array}$ \\
\hline 1 & 0.08 & 12 & 8 & clear \\
\hline 2 & 0.08 & 12 & 8 & $\begin{array}{l}\text { too viscous } \\
\text { to form gel }\end{array}$ \\
\hline 1 & 0.025 & 12 & 8 & clear \\
\hline 1 & 0.08 & 12 & $8^{a}$ & opaque \\
\hline
\end{tabular}

a mole ratio of isopropanol to $\mathrm{Ti}$

The influence of water concentration upon gelling volume and gelling time is shown in Table IX. In this table, the gelling volume is represented by the height of sample in a vial when the sample gelled, given that the initial height was $2.05 \mathrm{~cm}$ for every sample. Since the gelling volume reflects the degree and type of polymerization, these results indicate that no obvious changes occurred in the gels as the water content was increased. The decrease in gelling time associated with increased water concentration simply reflects the faster hydrolysis rates that would be expected when more water is present in these systems.

The type of alcohol used as the solvent for these reactions is important because the solvent affects the rates of hydrolysis and condensation. Of the alcohols studied, ethanol and isopropanol gave the best results. Ethanol 
Table IX. Influence of water concentration on gelation time and volume.

\begin{tabular}{ccccc}
$\begin{array}{c}\mathrm{H}_{2} \mathrm{O} / \mathrm{Ti} \\
\text { (mole ratio) }\end{array}$ & $\begin{array}{c}\mathrm{H}^{+} / \mathrm{Ti} \\
\text { (mole ratio) }\end{array}$ & $\begin{array}{c}\mathrm{C}_{3} \mathrm{H}_{7} \mathrm{OH} / \mathrm{Ti} \\
\text { (mole ratio) }\end{array}$ & $\begin{array}{c}\text { Gelling Time } \\
\text { (h) }\end{array}$ & $\begin{array}{c}\text { Gelling Volume } \\
(\mathrm{cm})\end{array}$ \\
\hline & 0.025 & 28 & & \\
1.0 & 0.025 & 28 & 19.5 & 1.80 \\
1.5 & 0.025 & 28 & 14.5 & 1.85 \\
2.0 & 0.025 & 28 & 11.0 & 1.90 \\
2.5 & 0.025 & 28 & 5.0 & 1.95 \\
3.0 & 0.025 & 28 & 4.0 & 2.00 \\
3.5 & 0.025 & 28 & 3.5 & 2.00 \\
4.0 & & & 1.5 & 2.00
\end{tabular}

appeared to be the best solvent to use for making transparent gels. However, the amount of nitric acid used did not significantly affect the properties of the polymeric gels. The acid simply provided the low pH condition necessary to prevent precipitation of titania particles during hydrolysis. It may also have acted as a catalyst.

b. Experimental Method - Particulate Sols

Particulate titania sols can be prepared simply by increasing the ratio of water to alkoxide. When a large excess of water is present, hydrolysis occurs as soon as the titanium tetra-15o-propoxide contacts the water. Under such hydrolysis conditions, titanium hydroxide precipitates are produced that contain large aggregates of primary particles (or possibly even aggregates of aggregates of primary particles). Although these aggregates are too large to produce either stable sols or useful membranes, the aggregates can be broken up by peptization with nitric acid. The data in Table $X$ illustrate the effect of varying the concentrations of the reagents on the proferties of the resulting sols. 
Table $X$. Influence of reactant concentrations on the properties of particulate titania sols.

\begin{tabular}{ccccc}
$\begin{array}{c}\mathrm{H}_{2} \mathrm{O} / \mathrm{Ti} \\
\text { (mole ratio) }\end{array}$ & $\begin{array}{c}\mathrm{H}^{+} / \mathrm{Ti} \\
\text { (mole ratio) }\end{array}$ & $\begin{array}{c}\mathrm{TiO}_{2} \\
(\text { wt } 8)\end{array}$ & $\begin{array}{c}\text { Stability } \\
\text { of Sol }\end{array}$ & $\begin{array}{c}\text { Particle Size } \\
\text { (nm) }\end{array}$ \\
\hline & 0.08 & 2.0 & NP & \\
200 & 0.2 & 2.0 & stable & 87 \\
200 & 0.4 & 2.0 & stable & 82 \\
200 & 0.7 & 2.0 & stable & 96 \\
200 & 1.0 & 2.0 & stable & 139 \\
200 & 1.2 & 2.0 & unstable & \\
200 & & & & \\
& 0.2 & 1.3 & stable & 79 \\
300 & 0.5 & 1.3 & stable & \\
300 & 1.0 & 1.3 & stable & \\
300 & 1.2 & 1.3 & stable &
\end{tabular}

a $\mathrm{NP}=$ sol that was not peptized completely, so large aggregates were still present.

$\mathrm{b}$ Average effective diameter as measured by quasi-elastic light scattering at several scattering angles. These particles may still be aggregated.

The data in Table $\mathrm{X}$ indicate that stable sols can be achieved if the molar ratio of $\mathrm{H}^{+}$(from the acid) to $\mathrm{Ti}$ is between 0.1 and 1.0 . This range can be expanded only by diluting the suspension. The increase in the average interparticle distance that accompanies dilution makes aggregation more difficult than in more concentrated sols. Coherent membranes could only be formed from stable sols. However, diluting the suspension has one drawback relative to the formation of unsupported membranes. Because of the large excess of water present in these systems, a large amount of solvent must be evaporated in order to form xerogels. Even at a $\mathrm{H}_{2} \mathrm{O}: \mathrm{Ti}$ mole ratio of $200: 1$, the original sol had to lose over $97 \%$ of its weight before it formed a xerogel.

It has been reported that the concentration of the inorganic acid used for peptization also affects the gelling volume of alumina gels [12]. Measurements of the gelling volume for these titania sols indicated that the 
gelling volume was a minimum when the $\mathrm{H}^{+}: \mathrm{Ti}$ mole ratio was near 0.4 . This value also corresponded to the condition in which the smallest particles were formed (as observed by quasi-elastic light scattering - QELS).

c. Effects of Hydrolysis and Peptization Conditions on the Particle Size of Titania Sols

Since we expect the pore size in the final membranes to be controlled by the particle size in the precursor sols, it is quite important to be able to control this particle size. The final particle size is determined by processing conditions during both hydrolysis and peptization. Two main experimental variables, $\mathrm{pH}$ and temperature, affect the nature of the precipitates produced during hydrolysis. The data in Table XI indicate that hydrolyzing acidic solutions at lower temperatures produced smaller particles in the final sol. This result can be attributed either to production of primary particles with smaller sizes or to breakup of loose agglomerate structures into smaller particles. Both the amount of acid utilized and the reaction temperature play important roles in determining the particle size that results from the combination of hydrolysis and peptization. Their effects are manifested via their influence on the rates of hydrolysis and condensation of the initial reactants, on the kinetics of nucleation and particle growth, and on agglomeration and precipitation processes.

Peptization also affects the nature of the particles in the final sols. This procedure involves three possible processes which can occur simultaneous1y: 1) break-up of agglomerates into particles with colloidal dimensions as a result of chemical, thermal and/or mechanical phenomena; 2) charging of par- 
Table XI. Effects of hydrolysis and peptization conditions on the properties of $\mathrm{TiO}_{2}$ colloidal sols ${ }^{\mathrm{a}}$.

\begin{tabular}{|c|c|c|c|c|c|c|}
\hline \multicolumn{2}{|c|}{$\begin{array}{l}\text { Hydrolysis } \\
\text { Conditions }\end{array}$} & \multicolumn{3}{|c|}{ Peptizing Conditions } & \multirow{2}{*}{$\begin{array}{c}\text { Particle } \\
\text { Diameter } \\
(\mathrm{nm})\end{array}$} & \multirow[t]{2}{*}{$\begin{array}{l}\text { Final pH } \\
\text { in Sols }\end{array}$} \\
\hline $\mathrm{pH}$ & Temp $\left({ }^{\circ} \mathrm{C}\right)$ & $\mathrm{pH}$ & Temp $\left({ }^{\circ} \mathrm{C}\right)$ & Time $(h)$ & & \\
\hline $6-7$ & $25 \pm 2$ & 0.99 & $85 \pm 2$ & 10 & $50 \pm 1$ & 1.14 \\
\hline 1.1 & $25 \pm 2$ & 1.1 & $85 \pm 2$ & 10 & $19 \pm 1$ & - \\
\hline $6-7$ & $65 \pm 2$ & 0.99 & $85 \pm 2$ & 10 & $123 \pm 1$ & 1.16 \\
\hline 1.12 & $25 \pm 2$ & 1.12 & $25 \pm 2$ & 66 & $16 \pm 1$ & 1.49 \\
\hline
\end{tabular}

all samples had a particle concentration of $22.2 \mathrm{~g} / \mathrm{L}$.

b All samples were sonicated under the same conditions before particle size measurements were made by QELS.

ticles by proton adsorption which, in turn, stabilizes the suspension through electrostatic repulsion effects; and 3) reaggregation of particles caused by collisions to give larger, but more weakly bound, aggregates whose dimensions remain in the colloidal range. The overall process of peptization is complex, and the average size of the particles present in the end product will depend on the relative rates of breakdown and aggregation processes. A number of factors affect these processes: 1) solution $\mathrm{pH}$ determines the surface potential of the particles; 2) ionic strength controls the thickness of the double layer surrounding the particles; 3) particle concentration affects the probability of collision; 4) temperature provides the energy for breaking apart precipitates and also for reaggregating the resultant particles.

Several $\mathrm{TiO}_{2}$ sols were prepared by peptization at $85^{\circ} \mathrm{C}$ for $10 \mathrm{~h}$ using different concentrations of nitric acid. Figure 9 indicates how the average particle size, measured by QELS, varied with the concentraticn of the peptizing acid (expressed in terms of the mole ratio of acid to titanium) for three sol concentrations. 


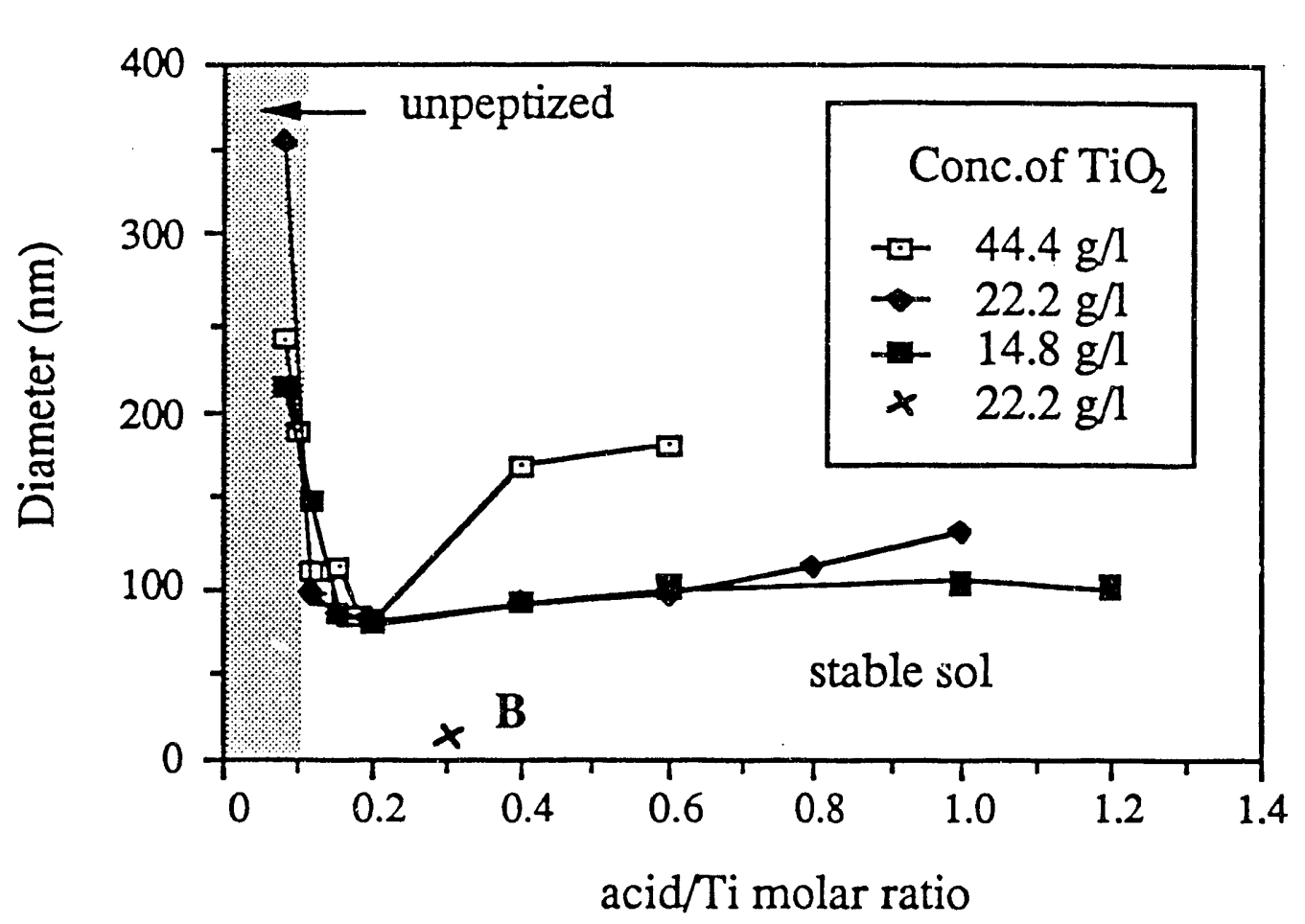

Figure 9. Average particle size as a function of amount of added acid in titania sols. The three curves correspond to the same hydrolysis conditions as the first sample in Table XI. Point B corresponds to the same hydrolysis conditions as the last sample in Table XI.

All three curves drop off rapidly as the $\mathrm{H}+/ \mathrm{Ti}$ mole ratio is increased from below 0.1 to 0.2 . They then pass through almost the same minimum point $(\mathrm{H}+/ \mathrm{Ti}=0.2$, diameter $=80 \mathrm{~nm})$ and then increase with increasing acid concentration. Similar behavior is also reflected in the particle size data shown in Table XI. These observations are readily explained by the DLVO theory of colloidal stability [13].

When the hydrolysis is performed in distilled water, the resulting $\mathrm{TiO}_{2}$ particles are essentially neutral (the isoelectric point for these particles is about $\mathrm{pH} 6.8$ ). Mutual attractions between the particles cause aggregates to form that have very large diameters (see the shaded region in Figure 9 corresponding to unpeptized systems). By adding acid to these systems, one 
causes a positive charge to develop on the particles. Mutual electrostatic repulsions begin to break apart any loosely aggregated flocs of particles. As more acid is added and the charges on the individual particles increase, the break-up of aggregates continues until a minimum particle size is reached.

Continued addition of acid past the point of minimum particle size will cause the particle size to increase because the particles now start to reaggregate. This effect is caused by the increase in the overall ionic strength of the solution as the acid concentration is increased. The extra ions being added to the system act to shield the electric fields of the charged particles from one another, thus neutralizing some of the electrostatic repulsion effects that serve to stabilize the sol. The van der Wals attractive forces between the particles then become more effective and cause particle reaggregation to occur. As one would expect, the actual effect is strongly dependent on the concentration of particles in the sol (see Figure 9).

Point B in Figure 9 represents the average particle size in a sol that was hydrolyzed in a strongly acidic medium and peptized at a lower temperature $\left(25^{\circ} \mathrm{C}\right)$ for 66 hours. The much lower particle size exhibited by this sol might be attributed either to the lower peptization temperature or the longer peptization time employed in preparing the sol. However, the second entry in Table XI shows that a sol with a small particle size can also be prepared at a much higher peptization temperature for a much shorter peptization time. The main reason for the small particle size is the strongly acidic hydrolysis conditions employed. When particles formed during hydrolysis under these conditions, the particles would immediately develop a large charge. These highly charged particles would not aggregate any further. Hence, larger particle sizes are not obtained. 
$\mathrm{X}$-ray diffraction experiments were used to determine crystalline states of precipitates obtained from $\mathrm{TiO}_{2}$ sols under various conditions of hydrolysis and peptization. Initially, all particles were amorphous, whether produced by hydrolysis in distilled water or acidified water at $25^{\circ} \mathrm{C}$ or in distilled water at $65^{\circ} \mathrm{C}$. (Precipitates obtained from polymeric sols, which were all alcoholic, were always amorphous.) Particles hydrolyzed at $65^{\circ} \mathrm{C}$ converted to anatase after aging at $85^{\circ} \mathrm{C}$ and remained as anatase through all further aging and peptization steps. Peptization of particles hydrolyzed at $25^{\circ} \mathrm{C}$ converted them to anatase. Particles hydrolyzed in distilled water and aged at $25^{\circ} \mathrm{C}$ eventually converted to anatase, but the time needed for this transition was not well defined. (It lies somewhere between 18 hours and 3 months.)

Once the particles had converted to anatase, their sizes were estimated from measurements of the extent of line broadening present in the X-ray diffraction spectra. Crystallite sizes were between 4.5 and $7 \mathrm{~nm}$. The smallest size corresponded to the sol hydrolyzed under acidic conditions and peptized at room temperature for 3 days. The sol prepared at high temperature was characterized by the largest crystallite size. These data again indicate the importance of preparing sols under acidic conditions if one desires to obtain small particles.

\section{d. Control of Particle Packing Density During Gelation}

Colloidal suspensions can be gelled either by evaporating the sol to remove solvent or by dialyzing the sol to remove the charge determining ions. These two different gelation methods gave different packing densities of particles and different gel structures. Evaporation promoted dense packing, 
while dialysis produced more open structures. Thus, these procedures can be used to tailor the porosity of membranes produced from particulate sols.

In the following discussion, two stages of the gelation process must be distinguished. When a sol is allowed to gel, the gel that first forms still contains a large amount of solvent. When the solvent is water, the gel that forms initially is called a hydrogel. At room temperature, solvent can continue to be removed from a hydrogel by evaporation or dialysis, producing a concomitant decrease in the volume of the hydrogel. When no more solvent can be removed from the gel at room temperature, the gel that remains is referred to as a xerogel. Note that xerogels still contain some solvent. We form unsupported membranes by firing xerogels to at least $200^{\circ} \mathrm{C}$. The amount of solvent remaining in the unsupported membranes depends on the firing conditions employed. (Studies performed using thermal analysis and Fourier transform infra-red spectroscopy indicate that solvent removal is one of several processes occurring in these membranes when they are heated from room temperature to between 200 and $500^{\circ} \mathrm{C}$. By $500^{\circ} \mathrm{C}$, removal of solvent and burnout of nitrates (from nitric acid) are complete [9, pp 142-148]).

\section{Formation of Dense Gels}

The effect of the size of the aggregates in the precursor sols on the properties of the $\mathrm{TiO}_{2}$ xerogels formed by evaporating these sols was studied. As initially prepared by peptization, the sols contained large aggregates (ca. $200 \mathrm{~nm}$ in diameter). These aggregates were broken down by sonicating the sols for different times. The sizes of the aggregates were then estimated hy the QELS technique. Evaporation of these sols led to the formation of xerogels 
whose pore structures were characterized via $\mathrm{N}_{2}$ adsorption-desorption measurements. This technique requires that, before the actual measurements can be performed, the xerogels must be outgassed at $200^{\circ} \mathrm{C}$ until all volatile species are removed.

Table XII contains a list of the properties of the xerogels derived from these titania sols. It is interesting to note that porosities and surface areas did not vary with aggregate size, as the differences reported in Table XII lie within the expected experimental error of the technique used. The particles in these highly charged systems seemed to pack very densely, giving porosities similar to those expected for randomly close-packed uniform spheres. Evaporation of these charge stabilized sols thus favored a dense packing of particles in the final membrane, regardless of the initial size of the aggregates in these sols.

Table XII. Characterization of titania sols and the corresponding xerogels produced by evaporation.

\begin{tabular}{ccc}
$\begin{array}{c}\text { Particle Diameter } \\
\text { in Sols } \\
\text { (in } \mathrm{nm} \text { ) }\end{array}$ & $\begin{array}{c}\text { Specific Surface Area } \\
\text { of Xerogels } \\
\left(\text { in } \mathrm{m}^{2} / \mathrm{g}\right)\end{array}$ & $\begin{array}{c}\text { Porosity } \\
\text { of Xerogels } \\
(8)\end{array}$ \\
\hline & & \\
$53 \pm 0.8$ & 203 & 33.6 \\
$110 \pm 0.7$ & 197 & 32.6 \\
$190 \pm 2$ & 213 & 34.7 \\
$210 \pm 6$ & 208 & 35.1 \\
$220 \pm 7$ & 196 & 32.0 \\
& 204 & 33.2
\end{tabular}

These results can be explained by considering the physical processes that occur when evaporation causes gelation. In these systems, the aggregated particles of titania are highly charged, since the $\mathrm{pH}$ of the sol is about 1.5 and the isoelectric point of titania particles varies from 4.5 to 7 , depending 
on the nature of the particles. As water evaporates from these sols, the proton concentration increases (causing the $\mathrm{pH}$ to continually decrease), the ionic strength of the sol also continually increases, and the particles are forced closer together. At some point, the particles approach one another closely enough that their repulsive electric fields interact strongly to hold the particles in position, thereby forming a hydrogel. (Many people believe that there must be interparticle contact to form a network of particles in order to form a hydrogel. We are not sure that direct contact between particles is necessary in these systems or if the electrostatic forces that are generated are sufficiently strong to hold the particles in a network in the absence of direct contact between particles.)

The aggregated particles in the sol are highly charged and continually moving about until the hydrogel forms. In many respects, then, formation of a hydrogel is similar to the process of particle-particle aggregation, a process that has both been modeled by several groups [14-16] and studied experimentally in oxide systems [17]. When highly charged particles aggregate, the sticking probability during collisions is very low due to electrostatic repulsion. Therefore, if several particles are held near one another, they move about before forming the final structure. Such structures tend to be relatively densely packed. This type of aggregation is termed reaction-limited aggregation. A similar process must have occurred in these sols, since the final xerogels had relatively low porosities.

Sols containing particles of different sizes formed hydrogels at different stages of evaporation. Thus, the volumes of these hydrogels differed. Sols containing larger particles were characterized by larger gelling volumes. However, even when the gelling volumes of the sols differed, the final 
xerogels had similar properties (see Table XII). This observation suggests that the aggregated particles in the sols were formed by reaction-limited aggregation processes. Since these processes produce compact aggregates, the pore sizes in these aggregates were small and very similar to one another. This observation also suggests that the primary particles that comprise these compact aggregates were both relatively spherical and monodisperse. During this study, we observed that sols containing smaller particles showed less shrinkage upon drying and that fewer cracks were present in the final xerogels. Thus, although the porosities and mean pore sizes of the various xerogels were not affected by variations in the size of the aggregated particles in the precursor sols, sols that contained smaller aggregated particles could be used to produce gels with superior macroscopic properties. Based on this observation, a protocol was developed for preparing crack-free xerogels from peptized titania sols. These sols were sonicated for several hours to produce smaller aggregates and then carefully evaporated.

Not all sols can be evaporated to produce xerogels. As evaporation occurs, the ionic strength in the remaining sol rises. This increase in ionic strength lowers the electrostatic repulsion barrier between the particles by shielding their electric fields. If the ionic strength in the original sol is sufficiently high, evaporation will cause coagulation, as evidenced by the formation of precipitates, rather than gelation. If these systems are evaporated to dryness, powders are obtained (see Table X). Thus, the concentrations of reactants must be controlled in order to obtain gels. 


\section{Formation of More Open Gels}

While evaporation of sols tends to produce dense gels, dialysis of sols leads to xerogels with more open structures. Dialysis simply involves containing the sol in a dialysis membrane (we employ Spectra-Por membranes with a molecular weight cutoff of 3500 ) and dialyzing the sol against distilled water. Since the particles of the sol are too large to pass through the membrane, the sol gradually loses the charge determining protons, as well as other ions, as dialysis proceeds. Thus, the $\mathrm{pH}$ of the sol gradually rises toward the isoelectric point of the titania particles, and the charges on those particles are slowly neutralized. Even though the ionic strength of the sol drops slowly, a pH value will be reached where the tightly aggregated particles in the sol will begin to aggregate or agglomerate further.

This process is similar to the phenomenon which occurs when a critical coagulation concentration (CCC) is reached. At the CCC of an inert electrolyte, a stable suspension suddenly destabilizes as its particles form large agglomerates $[18,19]$. However, there is a very important difference between these two phenomena. The CCC is determined by adding different amounts of an inert electrolyte to a stable suspension until a large decrease is noted in the time necessary for that suspension to settle. However, the electrolyte is added in an uncontrolled manner, so that destabilization of the sol and the concomitant formation of large agglomerates occur relatively quickly. In our systems, destabilization of the sol is mediated by the slow movement of ions through the dialysis membrane. Consequently, although the particles in the sol continue to agglomerate, this process occurs sufficiently slowly that the 
sol does not destabilize and form precipitates. Hence there is a marked difference in the rates at which the two aggregation phenomena occur.

The rapid aggregation of particles in a destabilized sol has been modeled in terms of a process called diffusion-limited aggregation [14,15]. In this process, particles are assumed to have a high sticking probability on collision. Thus, particles are not able to move much after they collide. Such systems produce aggregates with more open structures than those obtained via the reaction-1imited aggregation process.

In our application of this concept, no attempt was made to form gels within the dialysis membrane. Instead, sols were prepared by adding titanium

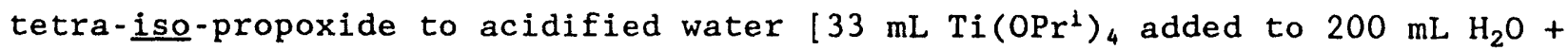
$1.43 \mathrm{~mL}$ conc $\mathrm{HNO}_{3}$ ] and stirring until peptized ( 3 days). These peptized sols were dialyzed for different lengths of time against deionized water, removed from the dialysis bag, and evaporated to form xerogels. These xerogels were fired to obtain membranes. In this procedure, dialysis caused agglomerates to form in the sol, but subsequent evaporation was responsible for causing the agglomerates to pack densely in the xerogel. Thus, the porosity of the xerogel was determined by the degree of agglomeration in the sol which was controlled, in turn, by the $\mathrm{pH}$ of the dialyzed sol.

Table XIII contains data obtained for sols dialyzed to different pH values. Examination of these data reveals that sols dialyzed to higher pH values produced larger aggregates and xerogels with higher porosities and greater specific surface areas. Bimodal pore size distributions were noted for xerogels obtained from sols that had been dialyzed to higher $\mathrm{pH}$ values. These data indicate that including a dialysis step ultimately leads to xerogels with more open structures than xerogels produced solely by evaporation. 
Table XIII. Characterization of titania sols and corresponding xerogels produced by dialysis.

\begin{tabular}{cccccc}
$\begin{array}{c}\text { pH of } \\
\text { Sol }\end{array}$ & $\begin{array}{c}\text { Particle Diam. } \\
\text { in Sols } \\
( \pm 0.05)\end{array}$ & $\begin{array}{c}\text { Specific } \\
\text { Surf Area } \\
\left(\mathrm{m}^{2} / \mathrm{g}\right)\end{array}$ & $\begin{array}{c}\text { Porosity } \\
(8)\end{array}$ & $\begin{array}{c}\text { Relative Height in PSD of } \\
0.7-0.8 \mathrm{~nm} \\
\text { Radius Peak }\end{array}$ & $\begin{array}{c}1.6-1.8 \mathrm{~nm} \\
\text { Radius Peak }\end{array}$ \\
\hline & & & & & \\
1.54 & $21 \pm 0.7$ & 111 & 24.1 & 5.7 & - \\
2.34 & $27 \pm 0.6$ & 270 & 38.5 & 20.0 & 22.1 \\
2.91 & $26 \pm 0.4$ & 329 & 46.9 & 29.4 & 42.4 \\
3.18 & $28 \pm 0.9$ & 348 & 50.2 & 25.9 & 68.4 \\
3.94 & $54 \pm 0.8$ & 375 & 54.0 & 18.9 &
\end{tabular}

Changes observed in the PSDs as the $\mathrm{pH}$ of the dialyzed sol was varied provide further information about the agglomeration processes. When the pH of the precursor sol was below 2.4, only small pores were obtained. (These pore sizes are near the limit of resolution of the technique.) This observation indicates that the particles remain too highly charged for agglomeration to be significant until the $\mathrm{pH}$ rises above 2.4 during dialysis. For sols with $\mathrm{pH}$ values below 2.4 , xerogel formation is essentially governed by the close packing of unagglomerated small particles, as noted earlier in the discussion of the formation of xerogels by evaporation. (The surprisingly low porosity obtained for the undialyzed sample at $\mathrm{pH} 1.54$ has yet to be explained.) When the sol was dialyzed to $\mathrm{pH}$ values above 2.4 , larger pores formed. The volume associated with these pores increased tremendously with increasing $\mathrm{pH}$. Concurrently, the volume associated with the smaller pores decreased. This change in the relative contributions to the pore volume with increasing $\mathrm{pH}$ indicates that the larger pores were formed in the more open structure of the agglomerates. As the $\mathrm{pH}$ was raised, fewer individual particles were present in the sol. Hence, fewer small pores were formed by close-packing of these small particles. One would expect to eventually reach a stage where all 
of the small particles had formed agglomerates so that no small pores would be observed in the PSD for the resulting xerogel. Our experimental conditions did not reach this stage.

One should note that, although the agglomerates that formed during dialysis were fairly open, the structures of these agglomerates were quite stable. When agglomerated sols were evaporated to form xerogels, the agglomerates were not forced back into a random close-packed structure with a porosity of 35 408. Moreover, the pore sizes observed in the xerogels did not vary with the extent of dialysis. This result suggests that the structure of the agglomerates is very uniform and independent of the degree of aggregation. More evidence for the stability of the agglomerate structure was obtained by firing the xerogel obtained from the sol that had been dialyzed to $\mathrm{pH} 3.94$. When this xeroge 1 was heated at $400^{\circ} \mathrm{C}$ for $30 \mathrm{~min}$, its surface area decreased to 180 $\mathrm{m}^{2} / \mathrm{g}$, but its porosity only decreased to 488 . This membrane still retained the relatively open structure characteristic of the original agglomerates in the sol. (The effect of firing temperature on the structures of these membranes is discussed further in Section IV.A.3.e.)

\section{Conclusions}

The structure of the final ceramic membrane is controlled to some extent by the structure of the aggregates which form during gelation of the original sol. The structure of the aggregates is controlled, in turn, by the nature of the particles and clusters that make up the sol. Highly charged particles tend to form relatively dense aggregates, since electrostatic repulsion forces between these particles reduce their sticking probabilities during aggrega- 
tion. On the other hand, weakly charged particles have high sticking probabilities and aggregate easily to form more open, ramified structures. These aggregate structures are reflected in the properties of the calcined ceramic membranes. Thus, one can control the porosity in the ceramic membrane to some extent without changing the pore size. This control requires appropriate regulation of the rates of evaporation and dialysis of the precursor sols.

\section{e. Effects of Sintering Conditions on the Properties of}

Unsupported Membranes

An unsupported $\mathrm{TiO}_{2}$ membrane was prepared from a particulate sol by hydrolyzing a system with a mole ratio of water to $\mathrm{Ti}$ of 200 and a mole ratio of $\mathrm{H}^{+}$to $\mathrm{Ti}$ of 0.4 , then carefully evaporating the product under controlled humidity conditions to form a gel. This gel was fired at successively higher temperatures. At each firing temperature, the surface area, percent porosity, and average pore radius were determined from $\mathrm{N}_{2}$ sorption measurements. Table XIV presents the results of this study. Note that the average pore radii were

Table XIV. Effects of firing temperature on the properties of unsupported titania membranes.

\begin{tabular}{|c|c|c|c|c|c|c|}
\hline $\begin{array}{l}\text { Firing } \\
\text { Temp } \\
\left({ }^{\circ} \mathrm{C}\right) \\
\end{array}$ & $\begin{array}{c}\text { Ramp } \\
\text { Rate } \\
\left({ }^{\circ} \mathrm{C} / \mathrm{min}\right) \\
\end{array}$ & $\begin{array}{c}\text { Dwe11 } \\
\text { Time } \\
\text { (h) } \\
\end{array}$ & $\begin{array}{c}\text { Porosity } \\
(8)\end{array}$ & $\begin{array}{c}\text { Specific } \\
\text { Area } \\
\left(\mathrm{m}^{2} / \mathrm{g}\right) \\
\end{array}$ & $\begin{array}{c}\text { Adsorption } \\
\text { Pore Radius } \\
\text { (nm) }\end{array}$ & $\begin{array}{c}\text { Desorpti } \\
\text { Pore Rad } \\
(\mathrm{nm})\end{array}$ \\
\hline 210 & 2 & 4 & 29.9 & 185 & - & 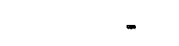 \\
\hline 325 & 2 & 4 & 31.7 & 128 & 1.9 & 1.8 \\
\hline 375 & 1 & 4 & 29.7 & 107 & 2.1 & 1.9 \\
\hline 425 & 1 & 4 & 26.6 & 79 & 2.4 & 1.9 \\
\hline 465 & 1 & 4 & 19.6 & 44 & 3.2 & 1.9 \\
\hline 500 & 1 & 4 & 8.9 & 11 & 3.5 & 2.0 \\
\hline
\end{tabular}

The PSD for the membrane fired at $210^{\circ} \mathrm{C}$ is characterized by a broad peak at the lower limit of resolution of the technique (see Figure 10). 
were determined from both adsorption and desorption isotherms. As noted in Section IV.A.1.b.1, we believe that the pore radius determined from the adsorption isotherm provides a more meaningful indication of the true mean pore size and the distribution of pore sizes.

Figure 10 shows PSDs calculated for three different sintering temperatures for this membrane. These data indicate that the pore size increased and the PSD broadened as the firing temperature increased. The plots in Figure 11 indicate that, as the sintering temperature increased, the average pore radius of the unsupported titania membrane increased, but that there was a significant decrease in the porosity at firing temperatures above $375^{\circ} \mathrm{C}$. Estimates of the crystal size obtained from measurements of the broadening of X-ray diffraction peaks in similar systems indicate that a significant increase in crystal size begins to occur at ca. $375^{\circ} \mathrm{C}$. This increase in crystal size is very likely associated with the decrease in porosity. These changes were arcompanied by concomitant decreases in the specific surface area.

Another important factor in determining the structure of the solid membrane is the form of titania present at each firing temperature. Thermal analysis studies indicated that, below $210^{\circ} \mathrm{C}$, titania was present mainly in an amorphous form. Between $210^{\circ} \mathrm{C}$ and $400^{\circ} \mathrm{C}$, transitions from amorphous titania to anatase were observed. However, it was difficult to use this technique to characterize these transitions in quantitative terms. Other processes (e.g., evaporation of water and decomposition of nitrates) occurred simultaneously and obscured the peak associated with the $\mathrm{TiO}_{2}$ phase transition. A further transition to rutile occurred at ca. $600^{\circ} \mathrm{C}$.

The form of titania obtained may also depend on the dwell time, i.e., the time for which the solid is held at a given temperature. The $\mathrm{TiO}_{2}$ sample 


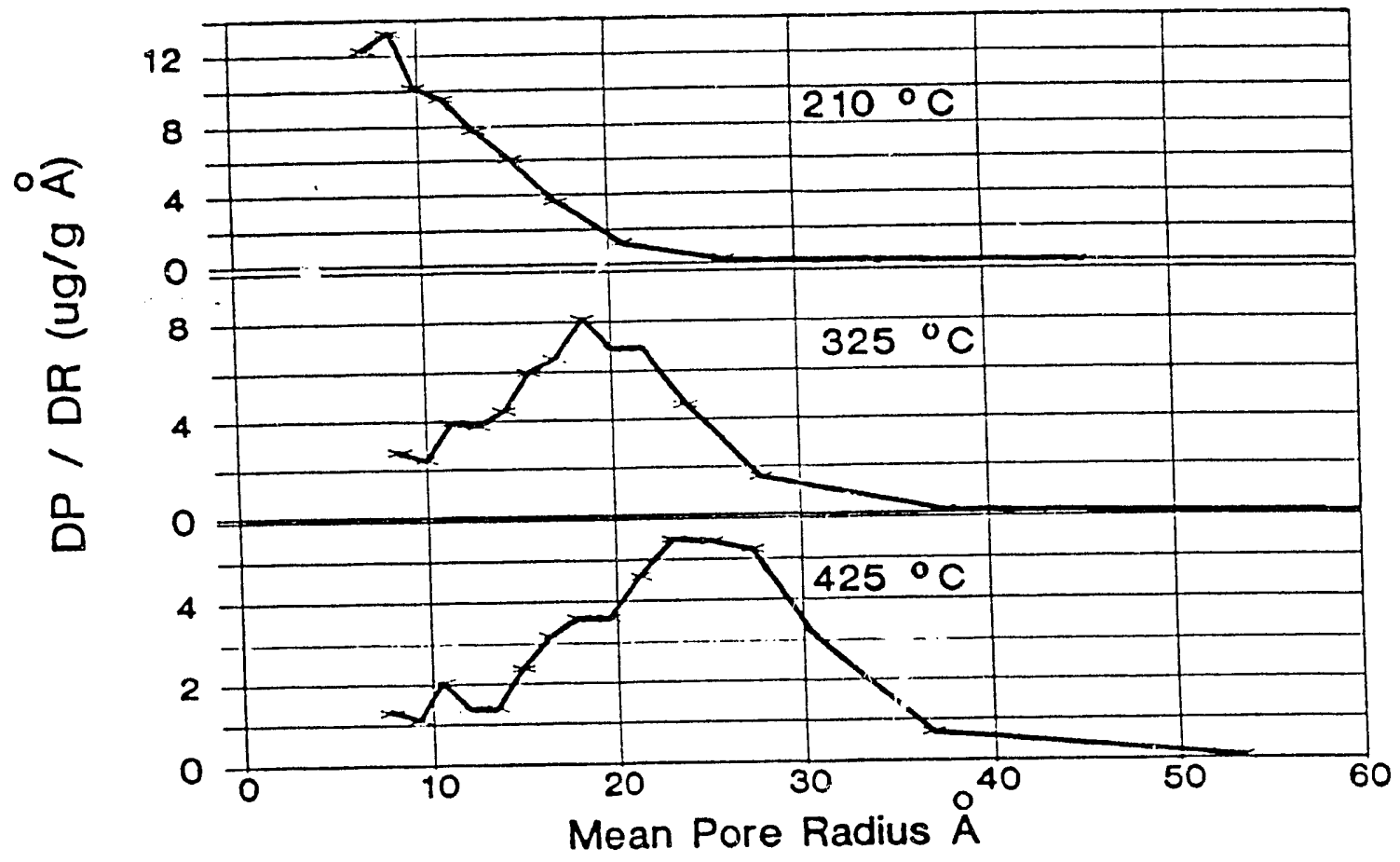

Figure 10. Pore size distributions :asured at three temperatures for an unsupported titania me" rane.

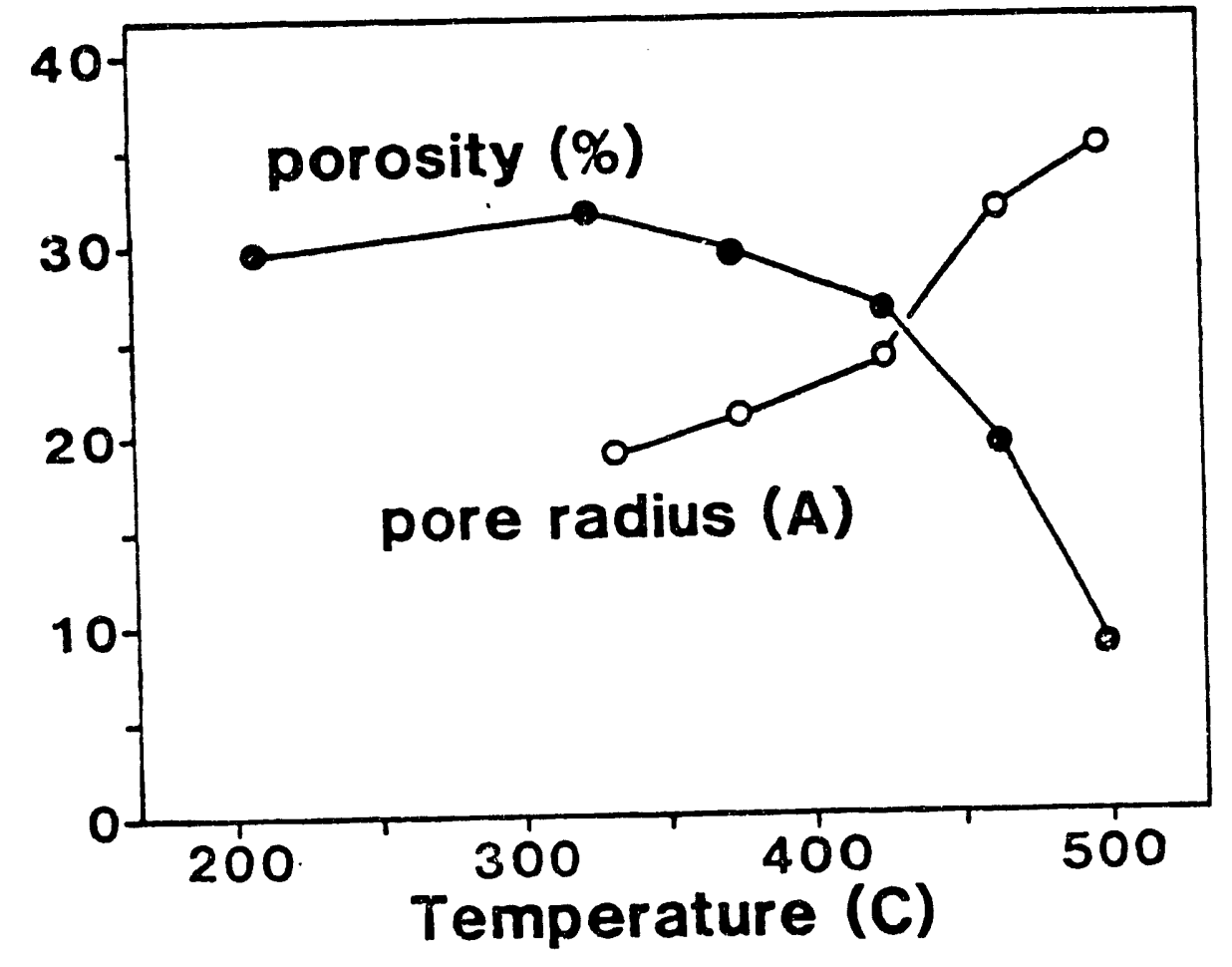

Tigure 11. Effect of sintering temperature on the mean pore size and the porosity of an unsupported titania membrane. 
used for the thermal analysis study was heated rather rapidly (at $\mathrm{ca} .10^{\circ} \mathrm{C} / \mathrm{min}$ up to $1000^{\circ} \mathrm{C}$ ) and no dwell times were employed. A corresponding study of $\mathrm{TiO}_{2}$ crystallinity was also performed using X-ray diffraction. Samples for this study were prepared by peptization and fired at different temperatures for 30 min. The results of this study are shown in Figure 12. Examination of these results reveals that even the xerogels contained small anatase particles, although much of the xerogel could still have been composed of amorphous titania. The transition to rutile started at $500^{\circ} \mathrm{C}$ and was completed by $600^{\circ} \mathrm{C}$. The latter temperature is lower than that observed in the thermal analysis study.

Further studies of the effect of the dwell time on the structure of the pores in titania membranes were performed by heating membranes from 10 min to $24 \mathrm{~h}$ at either 350 or $400^{\circ} \mathrm{C}$. When the structures of the pores in these membranes were analyzed by $\mathrm{N}_{2}$ sorption and $\mathrm{X}$-ray diffraction, the results shown in Figures 13 - 15 were obtained. An increase in the dwell time caused the crystal size to increase and both the surface area and porosity to decrease. The effect of an increase in the dwell time seemed to be more important at the higher temperature. There was no indication that any phase transitions had occurred in these systems.

Similar studies of the influence of the heating rate on the structure of the pores in these membranes showed much smaller effects than were observed in the dwell -ime studies. Significant changes were observed only for heating rates less than $1^{\circ} \mathrm{C} / \mathrm{min}$. Typically, we used heating rates of ca. $2^{\circ} \mathrm{C} / \mathrm{min}$ to prepare membranes for other studies, so this factor would have had little effect on those measurements. It should be noted that, when slow heating rates and long dwell times are used to prepare membranes, those conditions are 


\section{$\mathrm{TiO}_{2}$}

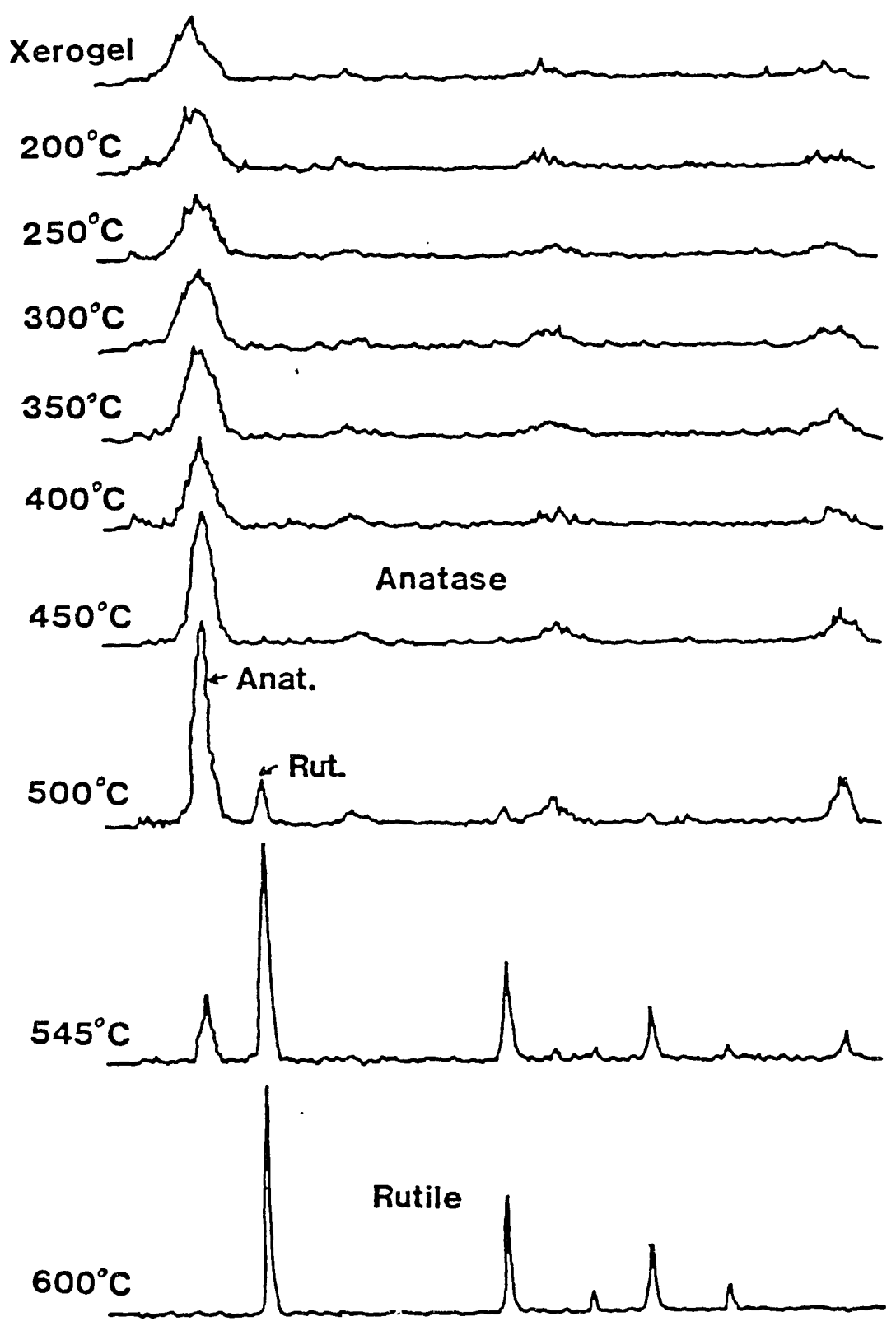

Figure 12. X-Ray diffraction patterns obtained from titania membranes formed from peptized sols. Each membrane was heated at $2^{\circ} \mathrm{C} / \mathrm{min}$ to the indicated temperature and held at that temperature for $30 \mathrm{~min}$. 


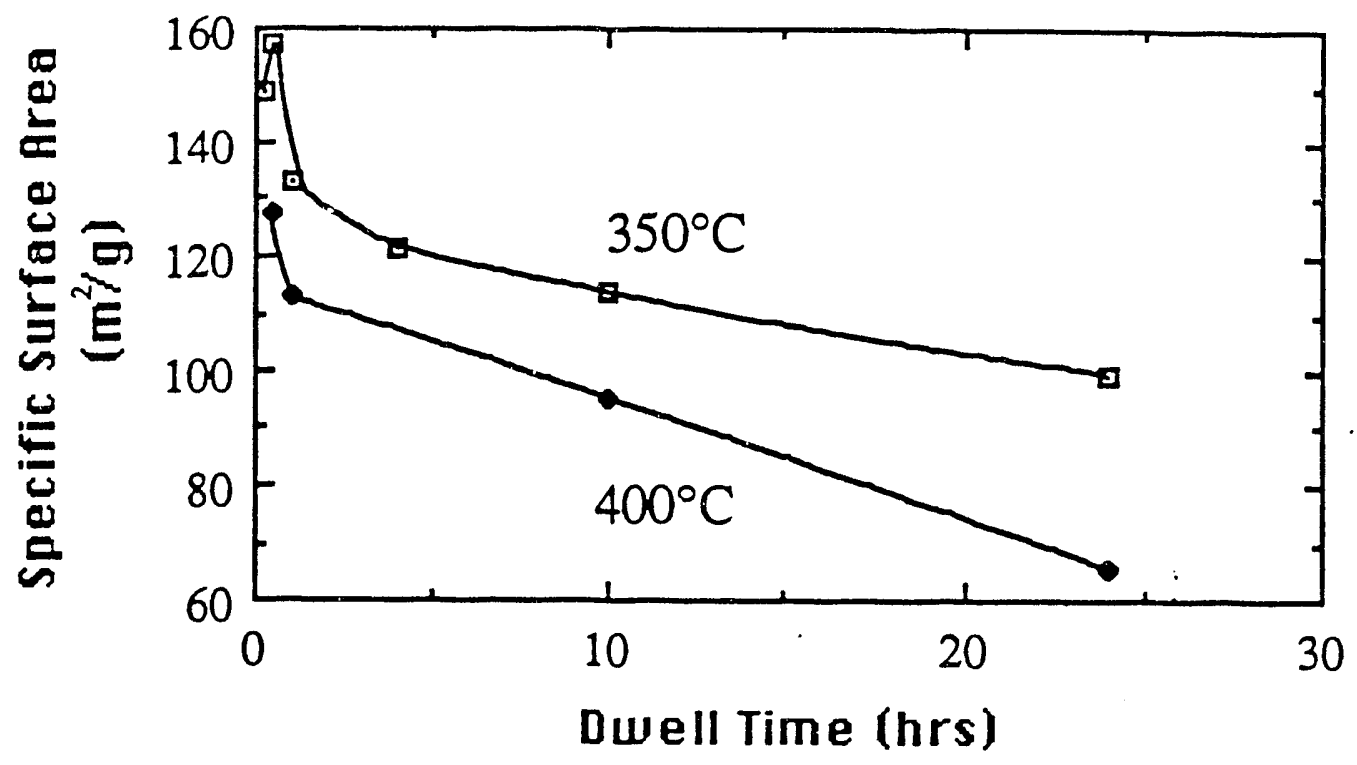

Figure 13. Effect of dwell time on the specific surface areas of $\mathrm{TiO}_{2}$ membranes fabricated from peptized sols. All membranes were heated at $2^{\circ} \mathrm{C} / \mathrm{min}$.

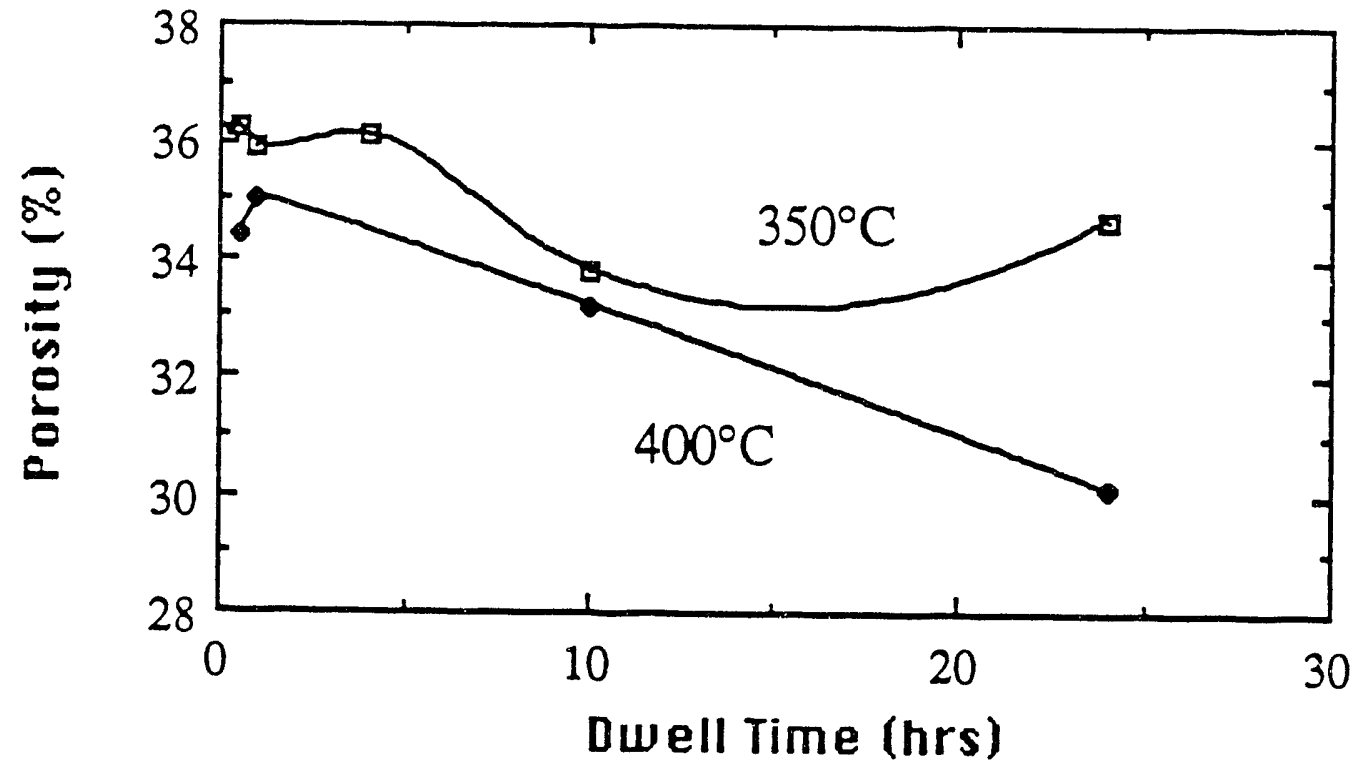

Figure 14. Effect of dwell time on the porosities of $\mathrm{TiO}_{2}$ membranes prepared from poptized sols. All membranes were healed at $2^{\circ} \mathrm{C} / \mathrm{min}$. 


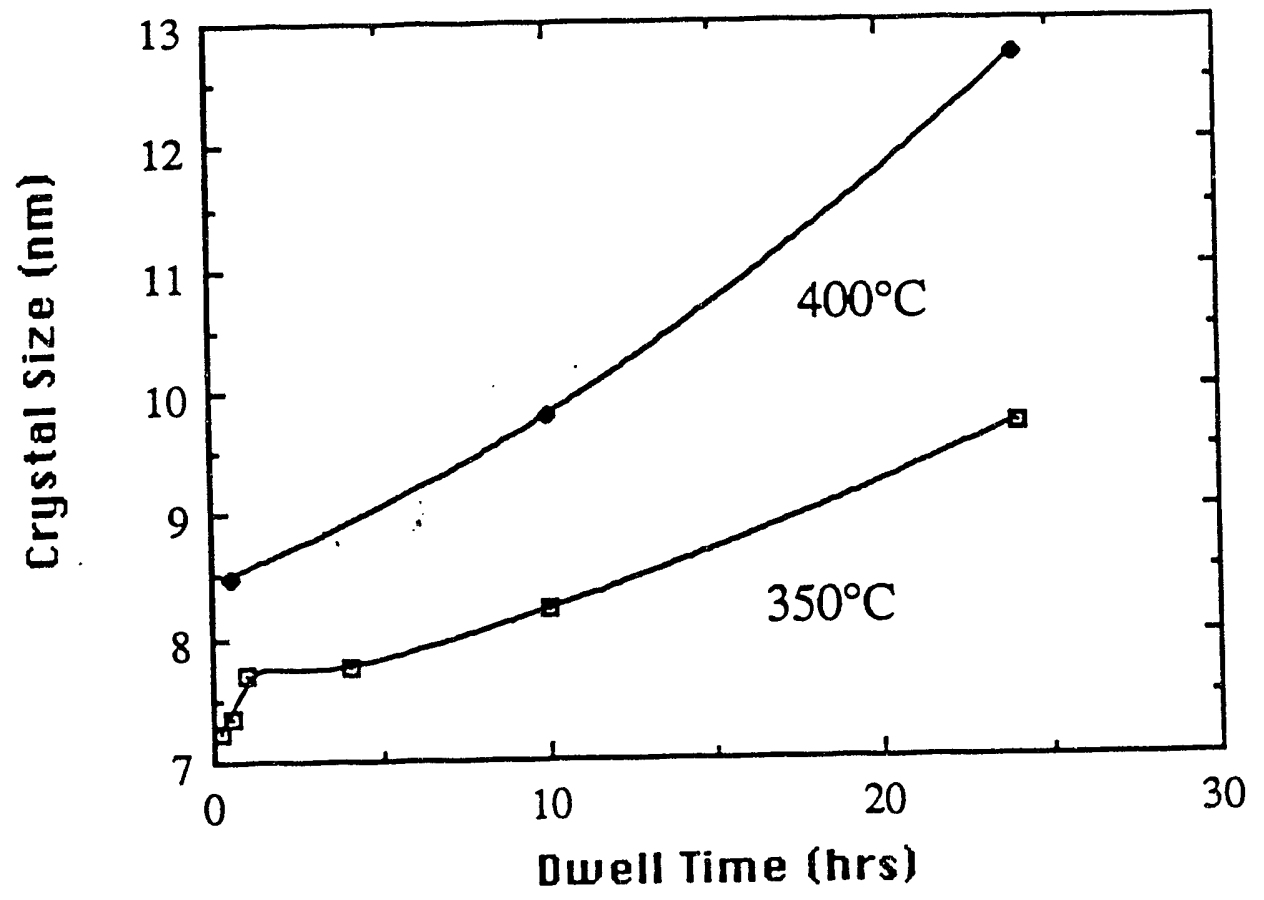

Figure 15. Effect of dwell time on the mean crystal size in $\mathrm{TiO}_{2}$ membranes prepared from peptized sols. All membranes were heated at $2^{\circ} \mathrm{C} / \mathrm{min}$.

conducive to accelerating particle growth which in turn results in decreases in both the specific surface areas and the porosities of the membranes.

We also investigated the effect of firing temperature on the pore structures of porosity controlled membranes prepared by dialysis of peptized sols to different $\mathrm{pH}$ values. Xerogels were prepared from these different sols and heated for $30 \mathrm{~min}$ at various temperatures up to $500^{\circ} \mathrm{C}$. Figures 16 and 17 illustrate the effects of sol pH and firing temperature on the porosities and crystal sizes, respectively, of the resulting membranes.

The effects of sol $\mathrm{pH}$ and firing temperature on the surface areas of the unsupported membranes were similar to the effects of those parameters on the porosities of the membranes. Both porosity and surface area increased as the sol pH increased, not only for the xerogel as shown in Table XIII, but also in 


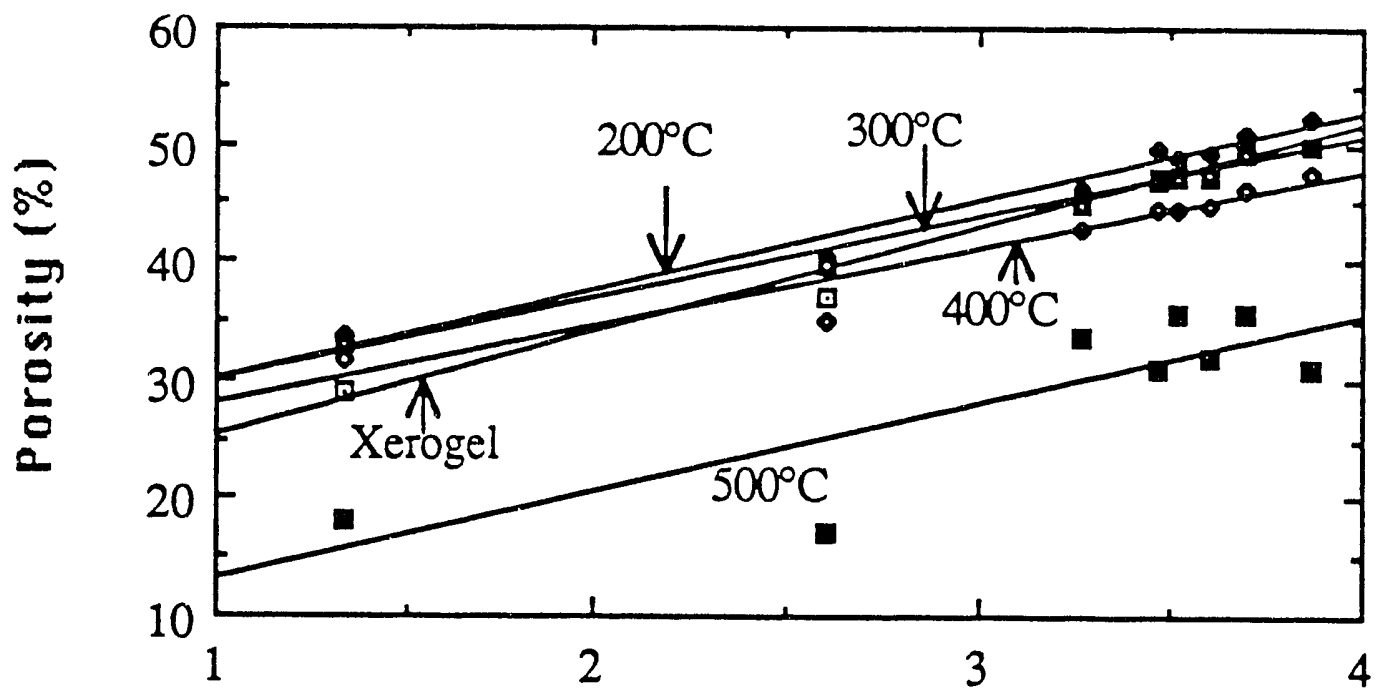

pH

Figure 16. Effects of $\mathrm{pH}$ and firing temperature on the porosities of $\mathrm{TiO}_{2}$ membranes. All membranes were heated at $2^{\circ} \mathrm{C} / \mathrm{min}$ to the indicated firing temperature and held at this temperature for $30 \mathrm{~min}$.

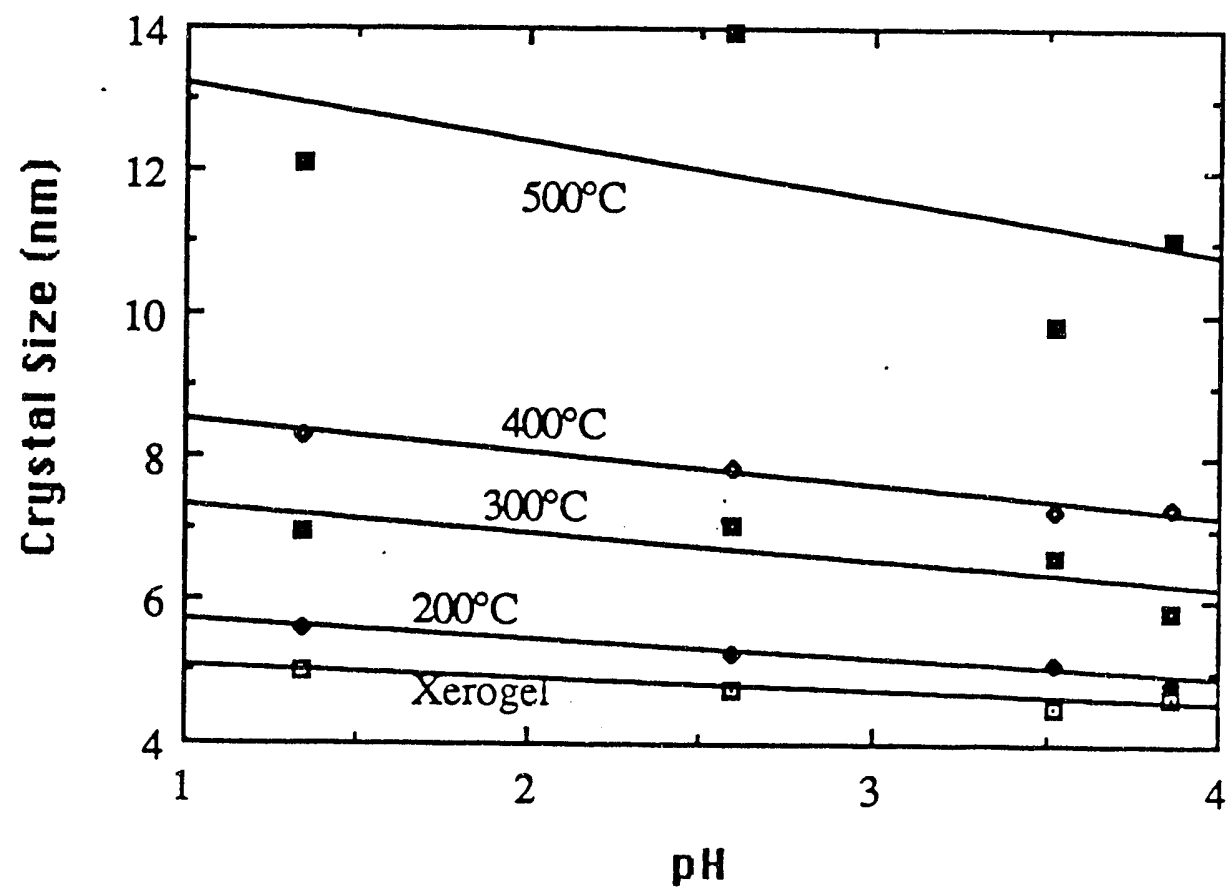

Figure 17. Effects of $\mathrm{pH}$ and firing temperature on the crystal sizes in $\mathrm{TiO}_{2}$ membranes. All membranes were heated at $2{ }^{\circ} \mathrm{C} / \mathrm{min}$ to the indicated firing temperature and held at this temperature for $30 \mathrm{~min}$. 
the membranes fired at a specified temperature. As expected, for a given $\mathrm{pH}$, both the porosity and the specific surface area decreased as the firing temperature increased. At a given firing temperature, crystal size decreased as the sol pH increased, while, at a given $\mathrm{pH}$, crystal size increased as the firing temperature increased. Note that, when membranes prepared at different $\mathrm{pH}$ values were fired to $500^{\circ} \mathrm{C}$ for $30 \mathrm{~min}$, the properties of these membranes were not identical. Evidently, the differences in the structures of the aggregates that form in the sols lead to different structures for the final calcined ceramic membranes.

f. Effects of Phosphate Addition on the Properties of Unsupported Membranes

Various concentrations of phosphoric acid were added "cold" (see Section IV.1.d for an explanation of this term) to a titania sol. A gel quickly formed when the final phosphoric acid concentration was $0.005 \mathrm{M}$ or higher. A coacervate also formed at a phosphoric acid concentration of $0.01 \mathrm{M}$. Stable sols were obtained only for phosphoric acid concentrations of $10^{-3} \mathrm{M}$ or lower. Xerogels formed from these sols appeared to be very similar to xerogels formed from titania sols that were identical in all respects except that they contained no phosphate. All of these xerogels cracked during evaporation.

One experiment was performed involving the "late" addition of phosphoric acid to give a phosphate concentration of $10^{-4} \mathrm{M}$. (Other acid concentrations were not used because the formation of stable sols in titania systems is much more sensitive to $\mathrm{pH}$ than is the case for alumina systems.) The overall behavior of this system was similar to that of the non-phosphated titania 
systems, except that, in the phosphated system, careful evaporation produced a crack-free xerogel. Since crack-free titania xerogels were also formed by other processes involving careful evaporation of a stable precursor sol, the use of phosphate to form crack-free xerogels was not pursued.

Thermal analysis was used to compare the behavior of these phosphated titania xerogels to similar non-phosphated xerogels. No noticeable differences in behavior were observed, although the phosphate did burn out of the xerogels within a fairly narrow temperature range below $300^{\circ} \mathrm{C}$.

In the proposal for this project, an experiment was described in which the $\mathrm{X}$-ray diffraction patterns of nonphosphated and phosphated goethite powders were compared. Both types of powders gave spectra characteristic of goethite, but the spectrum of the phosphated goethite was more intense. This result was interpreted as indicating that the phosphate caused more ordering in the goethite system than would usually be obtained. Similar experiments were performed using a commercial anatase powder. When the spectrum from the anatase itself was compared to that of the phosphated anatase, no differences were observed. X-ray diffraction spectra of nonphosphated and phosphated titania membranes fired at $400^{\circ} \mathrm{C}$ for 1 hour were also compared. In this case, the intensities of the X-ray diffraction lines for the phosphated membrane were about three times stronger than those of the same lines in the spectrum obtained from the non-phosphated membrane. Thus, ordering similar to that observed previously with goethite particles also occurred in these ceramic membranes, but at a lower level.

In general, the addition of phosphate to titania sols did not significantly improve such macroscopic properties as the amount of cracking observed in xerogels that were formed from these sols. The concentrations of phosphate 
added to these systems also had to be controlled carefully to avoid destabili$z$ ing the precursor titania sols. Thus, it was concluded that further studies of the effects of phosphate addition on the properties of titania membranes were not warranted. No $\mathrm{N}_{2}$ adsorption-desorption studies were performed on membranes prepared from $\mathrm{TiO}_{2}$ sols that contained added phosphate.

\section{g. Summary}

Protocols were developed for preparing both particulate and polymeric sols of titania. Polymeric sols were obtained by limiting the amount of water present in the system to an amount that would not completely hydrolyze the titanium alkoxide precursor. Particulate sols resulted when a large excess of water was used to hydrolyze the alkoxide. We have focused on preparing and characterizing particulate titania sols because we expect to obtain better control over the final structure of the membrane by using particulate sols.

In our initial studies, particulate titania sols (peptized sols) were synthesized by hydrolyzing the alkoxide with deionized water and peptizing the resulting suspension with various concentrations of nitric acid. The diameters of the particles produced by this method varied from 50 to $140 \mathrm{~nm}$. Particles with smaller diameters (ca. $20 \mathrm{~nm}$ ) were only obtained when the hydrolysis step itself was carried out under strongly acidic conditions (pH of 1.1). When particles form during hydrolysis under these conditions, the particles immediately develop a large charge. Since these highly charged particles do not aggregate any further, larger particle sizes are not obtained.

Pore structures (porosities, specific surface areas and pore diameters) of the resulting membranes were affected by the conditions employed for the 
hydrolysis step. However, the conditions employed for the peptization step appeared to have much less effect on the pore structures of the membranes. Xerogels formed from peptized sols had identical specific surface areas (ca. $200 \mathrm{~m}^{2} / \mathrm{g}$ ) and porosities (ca. 33.58), although the diameters of the precursor particles in these xerogels varied greatly (50 to $220 \mathrm{~nm}$ ). These differences in the diameters of the particles were obtained by varying the conditions employed to peptize the particles. Acidic hydrolysis of the alkoxide, however, resulted in sols in which the diameters of the particles were between 15 and $20 \mathrm{~nm}$. A xerogel produced from such a sol had a much smaller specific surface area $\left(110 \mathrm{~m}^{2} / \mathrm{g}\right)$ and porosity (248) than were observed in xerogels prepared from peptized sols.

If sols that were produced by acidic hydrolysis were dialyzed to different $\mathrm{pH}$ values, the pore structures of the resulting xerogels varied greatly. The xerogel described previously, with a specific surface area of $110 \mathrm{~m}^{2} / \mathrm{g}$ and a porosity of 248 , was fabricated from an undialyzed sol at $\mathrm{pH} 1.54$. A xerogel that was fabricated from a sol that had been dialyzed to $\mathrm{pH} 4$ was characterized by a specific surface area of $375 \mathrm{~m}^{2} / \mathrm{g}$ and a porosity of 548 . These differences in the pore structures of the xerogels are attributed to the amounts of charge present on the particles in the precursor sols. Particles in the undialyzed sol are highly charged and tend to form relatively dense aggregates and xerogels. Dialysis lowers the charge on the particles which increases their sticking probabilities on collision and allows them to form aggregates with more open structures. The structures of these aggregates, in turn, are reflected in the properties of the calcined ceramic membranes. 
The conditions utilized for calcining the xerogels affected the pore structures of the resulting unsupported membranes. As the firing temperature was increased, the porosities and the specific surface areas of the membranes decreased while their pore diameters increased. This effect was observed both in membranes that were fabricated from peptized sols (large particles) and in membranes prepared from dialyzed sols (small particles). When membranes that were fabricated from peptized sols were fired at temperatures above $450^{\circ} \mathrm{C}$, the pore diameters of these membranes were larger than $6 \mathrm{~nm}$. inlumina membranes had to be fired at temperatures higher than $900^{\circ} \mathrm{C}$ in order to obtain such large pore diameters.) As a result, such membranes were not considered to be good candidates for permselectivity studies. Membranes of this type which were calcined at temperatures up to $500^{\circ} \mathrm{C}$ were found to consist of anatase particles. Such membranes may be useful for applications in photocatalysis that require anatase crystals but do not require structures containing very small pore diameters.

The pore structures of unsupported membranes prepared from peptized sols were also dependent on the length of time (dwell time) that the membranes were held at a given firing temperature. An increase in the dwell time caused the average size of the crystals in the membranes to increase and both the specific surface area and the porosity to decrease. The effect of increasing the dwell time was more important at higher firing temperatures. A similar study of the influence of the rate of heating on the pore structures of these membranes demonstrated that significant changes were observed only for heating rates of less than $1{ }^{\circ} \mathrm{C} / \mathrm{min}$. Heating rates of $2^{\circ} \mathrm{C} / \mathrm{min}$ or higher were employed for most of the studies described in this report. 
In general, the addition of phosphate to titania sols had little effect on such macroscopic properties as the amount of cracking observed in xerogels formed from these precursors. However, the concentrations of phosphate added to these systems had to be controlled carefully to avoid destabilizing the precursor titania sols. (Similar observations had been made in studies of the influence of phosphate on the properties of alumina membranes.) Thus, it was concluded that, for the purposes of this research project, further studies of the effects of phosphate addition on the properties of titania membranes were not warranted. Since there were indications that the addition of phosphate to titania sols could have some beneficial effects (e.g., increased intensity of $\mathrm{X}$-ray diffraction spectra of phosphated membranes), we may perform further studies of these effects at some future date. 


\section{Other Materials}

Unsupported membranes composed of other materials have been prepared from conventional sols of zirconia and several mixed oxides, especially $\mathrm{TiO}_{2}$ $\mathrm{ZrO}_{2}$. These materials have been characterized primarily in terms of their thermal stabilities rather than via attempts to define the range of reactant concentrations necessary to fabricate these materials in useful forms.

\section{a. Zirconia}

Zirconia membranes were of interest because zirconia is highly insoluble in water down to a pH of about $2[20] . \mathrm{ZrO}_{2}$ membranes were synthesized by duplicating the peptization method used to prepare $\mathrm{TiO}_{2}$ membranes. However, zirconia precipitates proved to be more difficult to peptize than titania precipitates. Hence, higher acid concentrations and longer times were utilized for peptization of zirconia.

The starting material employed was zirconium tetra-n-propoxide as a $70 \%$ solution in n-propanol. $7.3 \mathrm{~mL}$ of this solution were added to acidified water $\left(100 \mathrm{~mL} \mathrm{H} \mathrm{H}_{2} \mathrm{O}+1.6 \mathrm{~mL}\right.$ conc $\mathrm{HNO}_{3}$ ) while stirring at room temperature. The resulting precipitate was heated to $90^{\circ} \mathrm{C}$ and held at that temperature for 3 days to give a sol with a $\mathrm{pH}$ of 1.03 .

Unsupported membranes prepared from this sol were heated to selected temperatures and held at that temperature for one hour. Figure 18 shows the effect of this treatment on the porosities and specific surface areas of these membranes. While the reason for the anomalous behavior at low temperatures is not known (it may be an artifact related to the preparation of samples for 


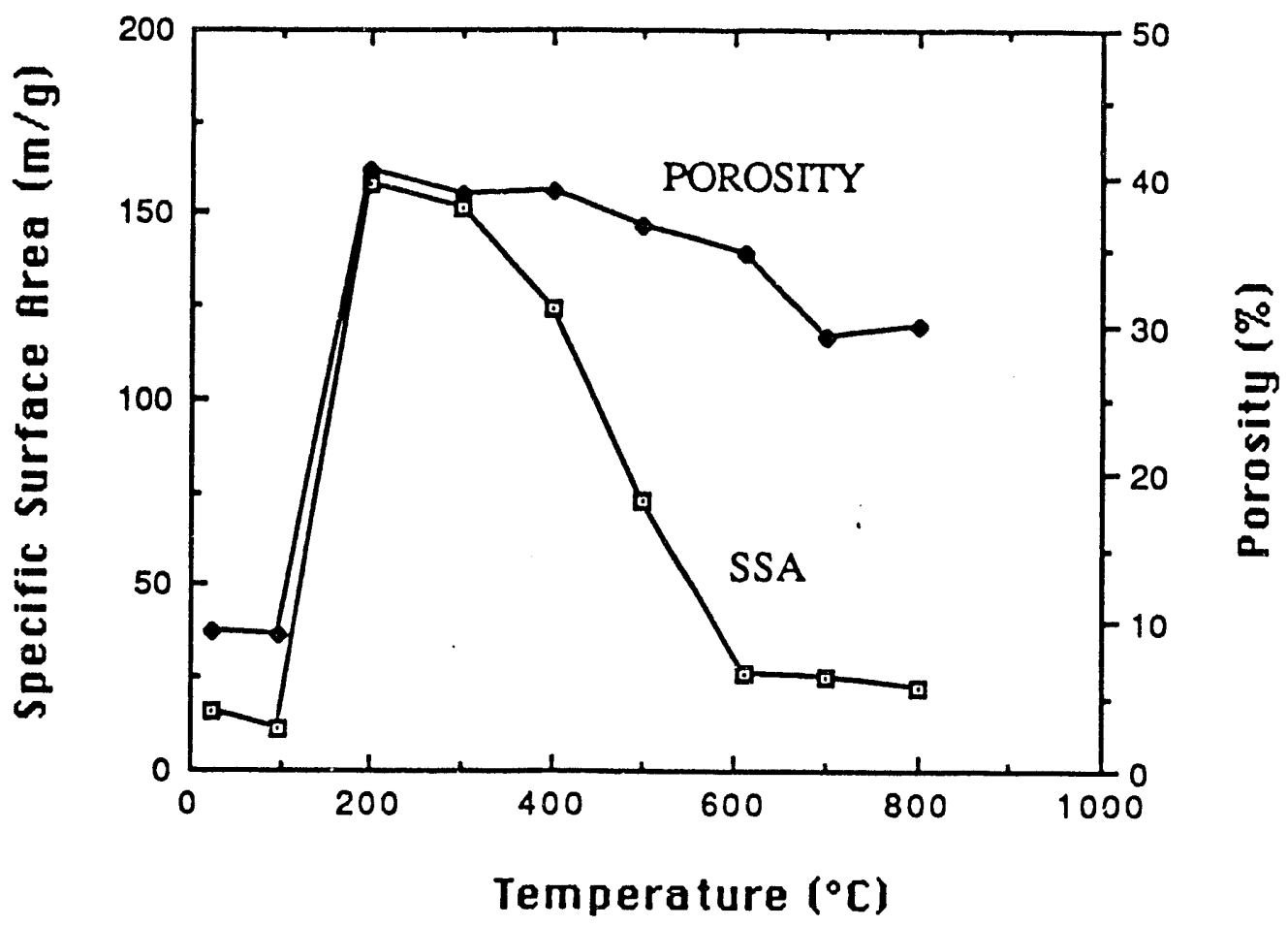

Figure 18. Effects of firing temperature on the specific surface areas and porosities of $\mathrm{ZrO}_{2}$ membranes prepared by peptization. A11 membranes were heated at $2^{\circ} \mathrm{C} / \mathrm{min}$ to the indicated firing temperature and held at this temperature for $1 \mathrm{~h}$.

study), it is clear that these membranes retained porosity even when fired to $800^{\circ} \mathrm{C}$, although their specific surface areas were quite low after firing to $600^{\circ} \mathrm{C}$. X-ray diffraction patterns for these materials are shown in Figure 19. Examination of these patterns indicates that the membranes are composed of amorphous material until a firing temperature of $400^{\circ} \mathrm{C}$ is reached. At this temperature, monoclinic crystals of zirconia begin to form. Formation of monoclinic zirconia may be related to the decrease in specific surface area shown in Figure 18, but it does not appear to be correlated with the changes in porosity.

Studies were performed to determine the effect of dialyzing conventional sols of zirconia on the properties of xerogels fabricated from these dialyzed sols. (Similar studies on titania sols are described in Section IV.A.3.d.) 


\section{$\mathrm{ZrO}_{2}$}

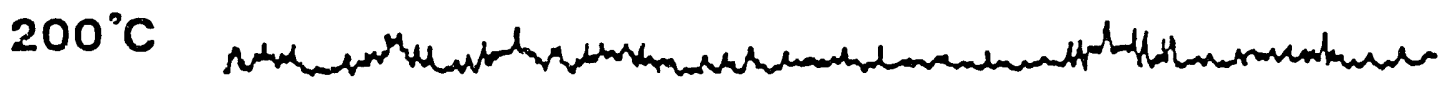

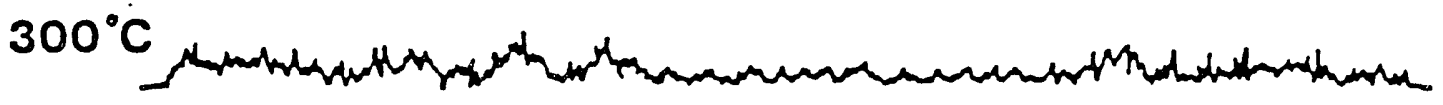

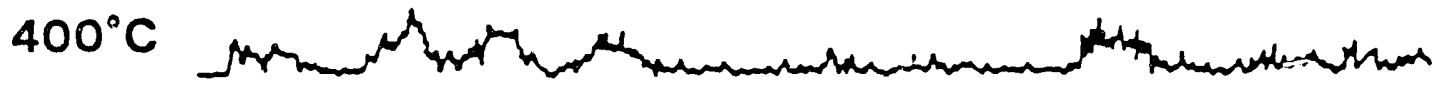

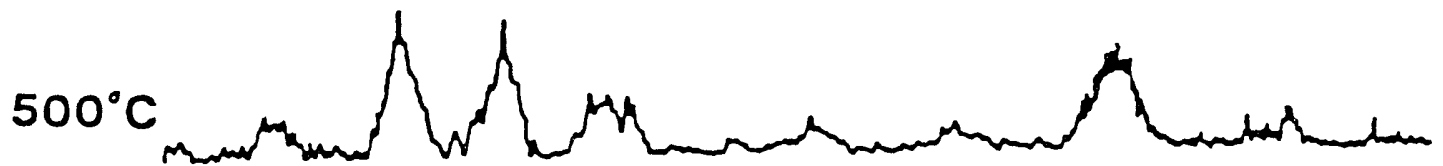

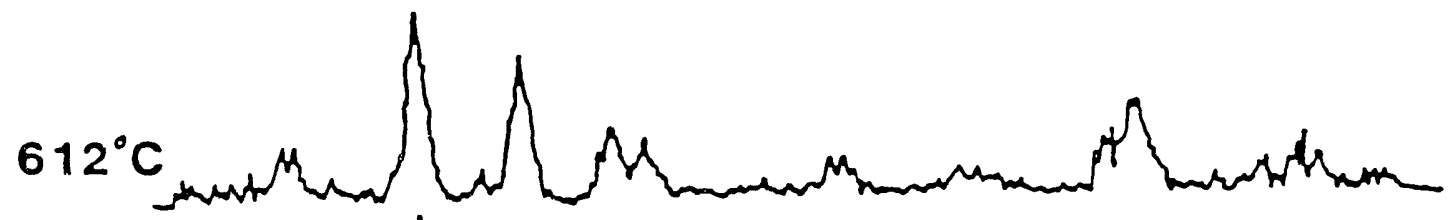

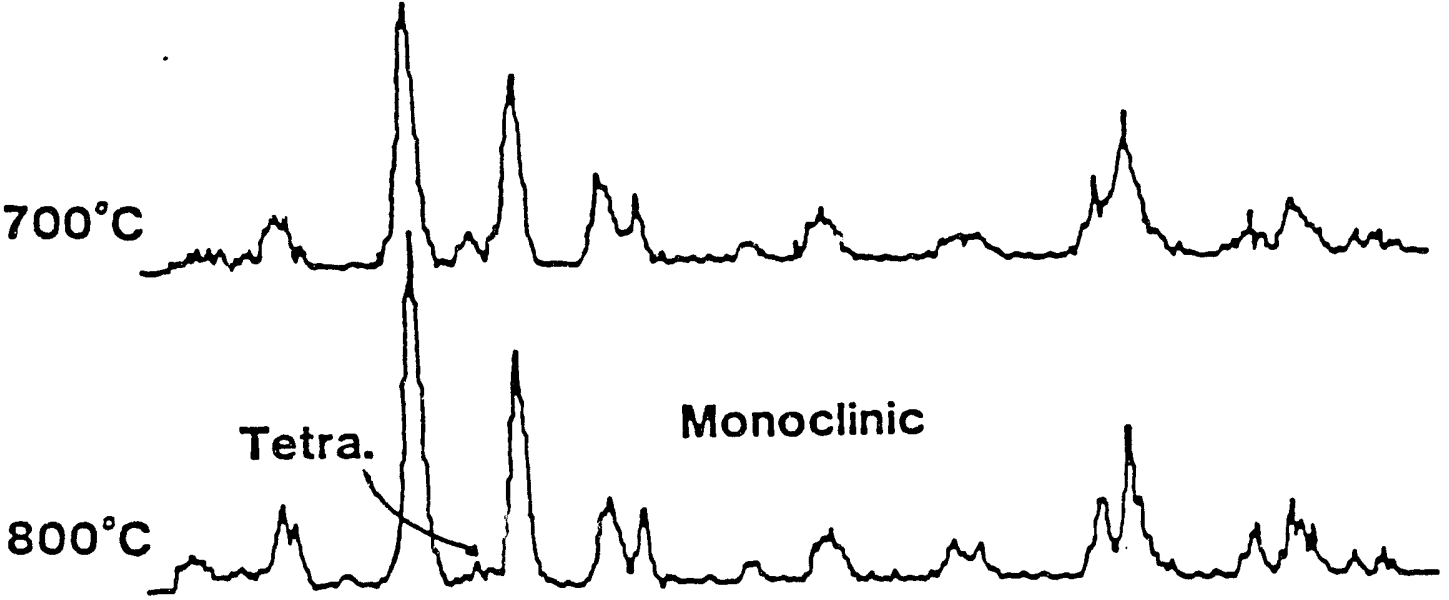

Figure 19. X-Ray diffraction patterns for $\mathrm{ZrO}_{2}$ membranes prepared by peptization. All membranes were heated at $2^{\circ} \mathrm{C} / \mathrm{min}$ to the indicated firing temperature. Dweli times are not known. 
Table XV contains data for the specific surface areas and porosities of unfired xerogels as a function of the $\mathrm{pH}$ of the precursor sol. These xerogels were outgassed at $200^{\circ} \mathrm{C}$. Dense, compact xerogels with very low porosities were formed from sols that were held at $\mathrm{pH}$ values below 5 . Xerogels with appreciable porosities could only be formed from sols that had been dialyzed to a $\mathrm{pH}$ above 6 . The maximum porosity was observed for the xerogel formed from the sol that had been dialyzed to $\mathrm{pH} 6.9$. This $\mathrm{pH}$ is very close to the measured isoelectric $\mathrm{pH}$ for these zirconia sols (ca. 7.1). Pore size distributions were also obtained for these xerogels, but no pores with radii larger than $0.8 \mathrm{~nm}$ (the lower limit of resolution of the technique) were observed.

Table XV. Characterization of xerogels produced by dialyzing zirconia sols prepared by peptization.

\begin{tabular}{ccc}
$\begin{array}{l}\mathrm{pH} \text { of Pre- } \\
\text { cursor Sol }\end{array}$ & $\begin{array}{c}\text { Specific Surface Area } \\
\left(\mathrm{m}^{2} / \mathrm{g}\right)\end{array}$ & $\begin{array}{c}\text { Porosity } \\
(8)\end{array}$ \\
\hline 4.99 & 9 & 7.1 \\
6.23 & 76 & 21.6 \\
6.62 & 121 & 29.2 \\
6.90 & 140 & 33.8
\end{tabular}

b. Mixtures of Titania and Zirconia

In general, metal alkoxides are mutually soluble and miscible in their parent alcohol. Thus, the starting material for a mixed oxide membrane can usually be prepared simply by adding appropriate amounts of the metal alkoxides to an alcoholic solvent with stirring. The resulting solution should be stirred for at least one hour to ensure complete mixing and to allow the alkoxides to react if such reactions are feasible. 
Titanium and zirconium isopropoxides were used as starting materials for synthesizing mixed $\mathrm{TiO}_{2}-\mathrm{ZrO}_{2}$ membranes. After the starting materials were mixed, they were reacted with $0.1 \mathrm{M} \mathrm{HNO}_{3}$ at room temperature. The resulting precipitates were then stirred for $30 \mathrm{~min}$. This suspension was heated to $85^{\circ} \mathrm{C}$ and held at this temperature for $10 \mathrm{~h}$. Ceramic membranes could be formed from this peptized sol by evaporation.

These membranes were characterized by $\mathrm{X}$-ray diffraction and $\mathrm{N}_{2}$ sorption studies. Figure 20 depicts the ultimate porosities and mean pore diameters of $258 \mathrm{ZrO}_{2}-\mathrm{TiO}_{2}$ membranes as functions of the firing temperature employed. Pore diameters of these membranes increased nearly linearly with firing temperature while porosities remained constant at firing temperatures up to $700^{\circ} \mathrm{C}$. Thus, by selecting an appropriate firing temperature, one can vary the pore diameter of a membrane from 1.5 to $5 \mathrm{~nm}$ without changing the porosity of the membrane.

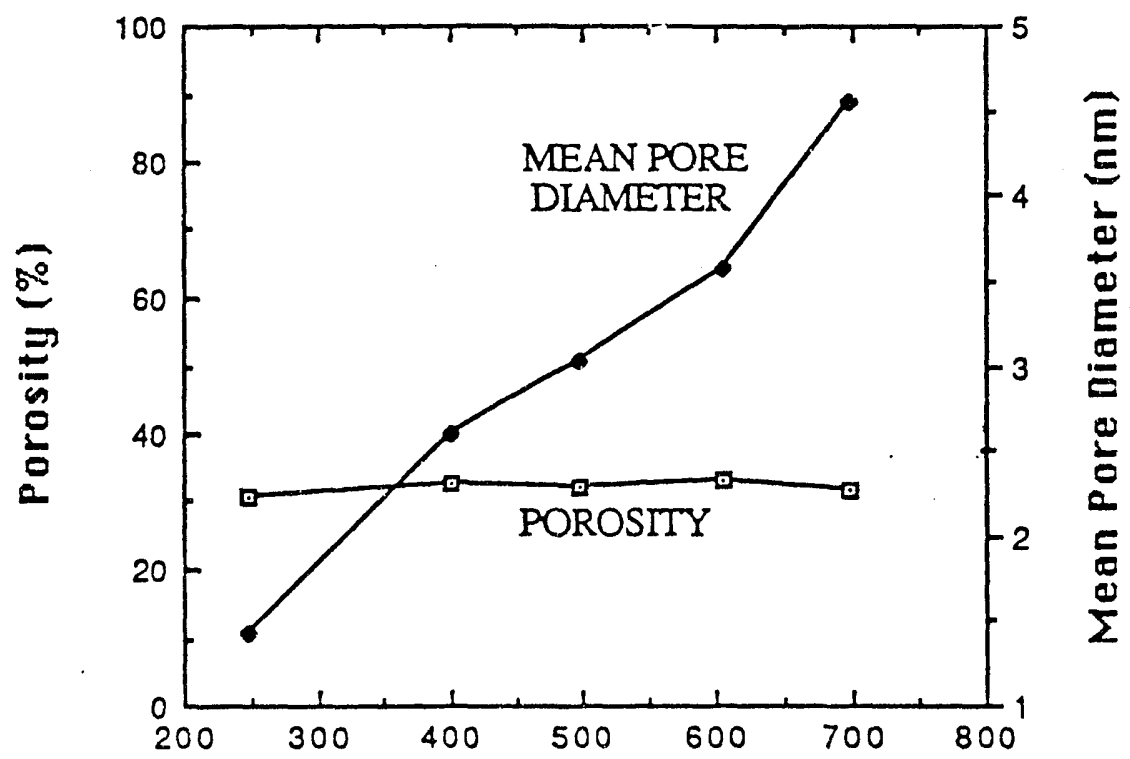

Temperature $\left({ }^{\circ} \mathrm{C}\right)$

Figure 20. Effects of firing temperature on the porosities and mean pore diametris of $258 \mathrm{ZrO}_{2}-758 \mathrm{TiO}_{2}$ membranes prepared by peptization. All membranes were heated at $2^{\circ} \mathrm{C} / \mathrm{min}$ to the indicated firing temperature and held at this temperature for $30 \mathrm{~min}$. 
Inspection of Figures 21 and 22 permits one to compare the thermal stabilities of pure $\mathrm{TiO}_{2}, 108 \mathrm{ZrO}_{2}-\mathrm{TiO}_{2}$ and $258 \mathrm{ZrO}_{2}-\mathrm{TiO}_{2}$ membranes. The thermal stabilities of the mixed oxide membranes represent significant improvements over the thermal stability of the pure $\mathrm{TiO}_{2}$ membrane. Even after firing at $800^{\circ} \mathrm{C}$, the mixed oxide membranes retained $30 \%$ porosity, although their effective surface areas were very low. Pure $\mathrm{TiO}_{2}$ membranes suffer essentially complete loss of porosity by firing to only $600^{\circ} \mathrm{C}$.

$\mathrm{X}$-ray diffraction studies on the mixed oxide membranes indicated that anatase was the only crystalline phase present, even in membranes fired to $800^{\circ} \mathrm{C}$. No evidence was found for a separate $\mathrm{ZrO}_{2}$ phase in any membrane, including membranes that contained $258 \mathrm{ZrO}_{2}$. We attribute the enhanced thermal stabilities of the mixed oxide membranes to the formation of a solid solution of $\mathrm{TiO}_{2}$ and $\mathrm{ZrO}_{2}$. This solid solution delays the phase transition from anatase to rutile in the $\mathrm{TiO}_{2}$ membrane, a transition that corresponds to the closing of the pores in $\mathrm{TiO}_{2}$ membranes. The solid solution also inhibits densification and particle growth until higher firing temperatures are reached.

\section{c. Other Mixed Oxides}

Two other mixed oxide membranes were prepared from conventional sols: $108 \mathrm{Fe}_{2} \mathrm{O}_{3}-\mathrm{TiO}_{2}$ and $58 \mathrm{Mb}_{2} \mathrm{O}_{5}-\mathrm{TiO}_{2}$. Details of their syntheses can be Eound in reference 9, pp. 225-226. These materials were synthesized for possible applications in photocatalytic processes and were not. well characterized. Xray diffraction measurements of the $\mathrm{Fe}_{2} \mathrm{O}_{3}-\mathrm{TiO}_{2}$ membranes indicated that only crystalline phases associated with titania were present. These studies also 


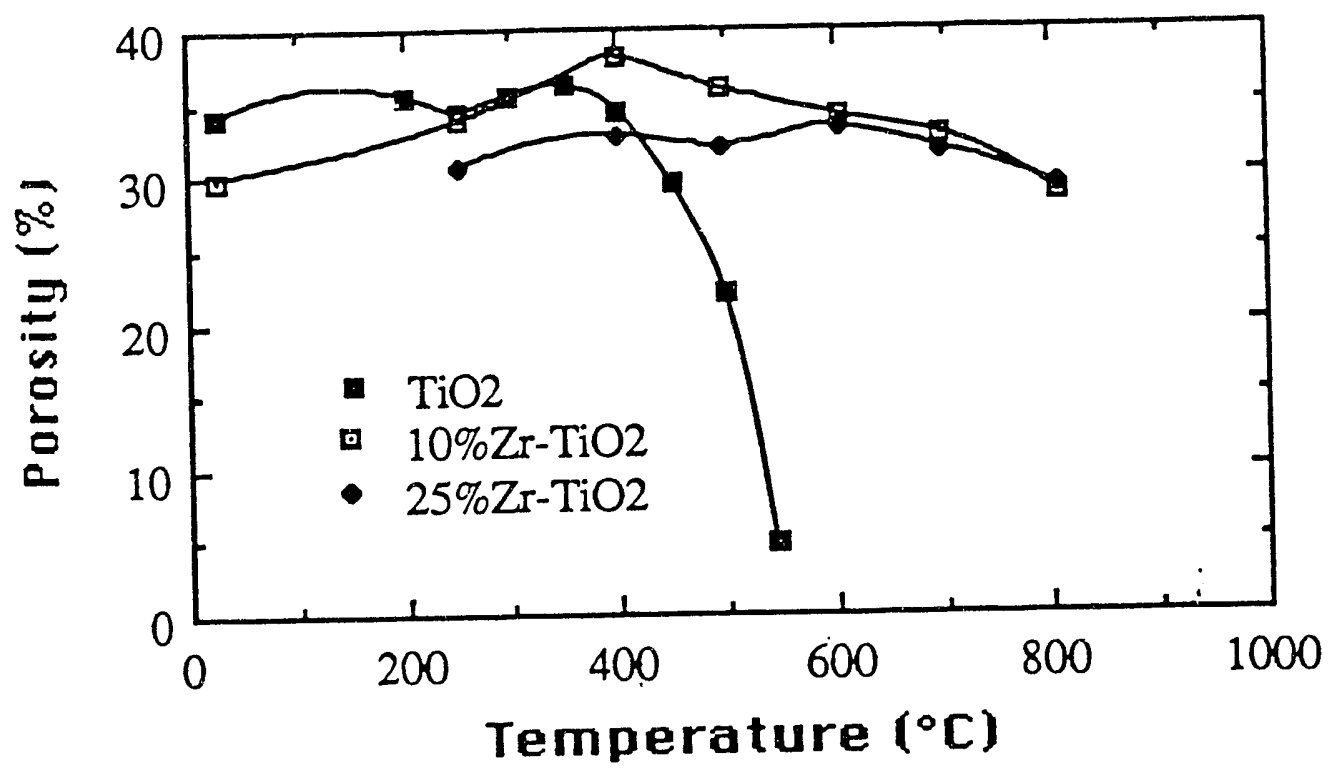

Figure 21. Effect of firing temperature on the porosities of pure $\mathrm{TiO}_{2}$ and mixed $\mathrm{TiO}_{2}-\mathrm{ZrO}_{2}$ membranes prepared by peptization. All membranes were heated at $2^{\circ} \mathrm{C} / \mathrm{min}$ to the indicated firing temperature and held at this temperature for $30 \mathrm{~min}$.

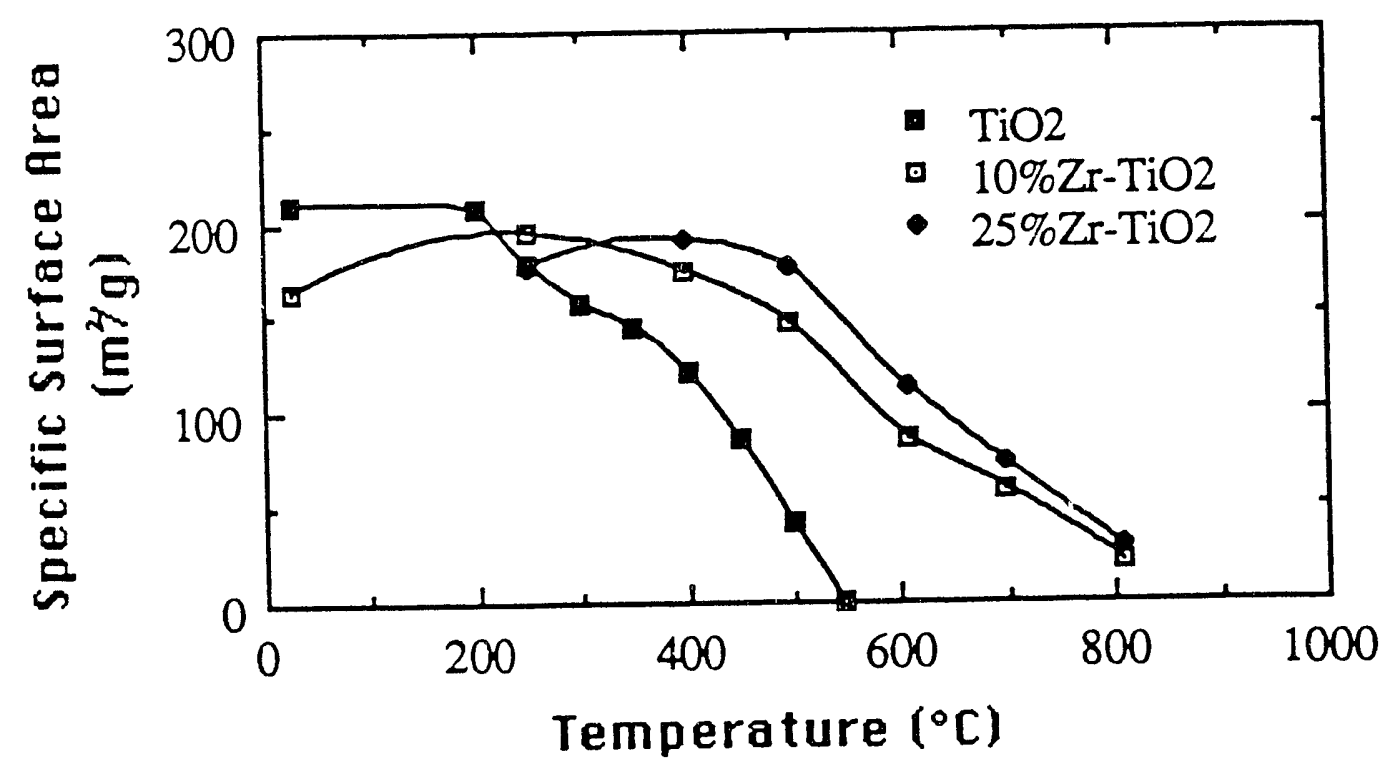

Figure 22. Effect of firing temperature on the specific surface areas of pure $\mathrm{TiO}_{2}$ and mixed $\mathrm{TiO}_{2}-\mathrm{ZrO}_{2}$ membranes prepared by peptization. All membranes were heated at $2^{\circ} \mathrm{C} / \mathrm{min}$ to the indicated firing temperature and held at this temperature for $30 \mathrm{~min}$. 
indicated that the anatase to rutile conversion began at $650^{\circ} \mathrm{C}$, a temperature that is $150^{\circ} \mathrm{C}$ higher tian the onset of the same transition in pure titania membranes.

\section{d. Summary}

Limited attention was devoted to fabricating ceramic membranes from conventional sols of other materials. Zirconia membranes were prepared and were shown to retain porosities of ca. $30 \%$ when fired to temperatures as high as $800^{\circ} \mathrm{C}$. However, the same membranes displayed very low specific surface areas (ca. $20 \mathrm{~m}^{2} / \mathrm{g}$ ) after firing to only $600^{\circ} \mathrm{C}$. While the xerogels of $\mathrm{zir}$ conia were amorphous, they began to form monoclinic crystals of zirconia at a temperature of $400^{\circ} \mathrm{C}$. In addition, the pore structures (porosities, pore diameters and specific surface areas) of these zirconia xerogels could be varied by dialyzing the precursor sols. Xerogels with porosities of ca. $30 \%$ and specific surface areas above $100 \mathrm{~m}^{2} / \mathrm{g}$ could only be formed by dialyzing the precursor sols to $\mathrm{pH}$ values greater than 6.5. Pore diameters of these xerogels were smaller than $16 \AA$.

Several mixed oxide membranes $\left(\mathrm{ZrO}_{2}-\mathrm{TiO}_{2}, \mathrm{Fe}_{2} \mathrm{O}_{3}-\mathrm{TiO} \mathrm{O}_{2}\right.$ and $\left.\mathrm{Nb}_{2} \mathrm{O}_{5}-\mathrm{TiO}_{2}\right)$ were also prepared. The mixed $\mathrm{ZrO}_{2}-\mathrm{TiO}_{2}$ membranes were characterized by determining the effects of firing temperature on their pore structures. These mixed oxide membranes maintained relatively high porosities and large specific surface areas at firing temperatures between 600 and $800^{\circ} \mathrm{C}$. In pure $\mathrm{TiO}_{2}$ membranes, similar properties could be observed at firing temperatures no higher than $450^{\circ} \mathrm{C}$. There was also evidence indicating that mixed $\mathrm{Fe}_{2} \mathrm{O}_{3}-\mathrm{TiO}_{2}$ membranes were characterized by a greater thermal stability than pure $\mathrm{TiO}_{2}$ membranes. 
B. Ceramic Membranes Formed from Nanoparticulate Sols

This research is based on the hypothesis that smaller particles would be formed in sols if the rate of hydrolysis of the precursor alkoxide solutions could be reduced. Two approaches have been employed to decrease the rate of hydrolysis: solubility control in aqueous systems and steric hindrance in alcoholic solvents. These approaches were applied primarily in studies of titania and mixed titania-zirconia systems, but a few studies of pure zirconia systems were also performed.

\section{Solubility Control}

\section{a. Explanation of the Technique}

This method requires that initial hydrolysis of a metal alkoxide precursor be performed under conditions that ensure the dissolution of the products and the formation of a solution of metal ions (e.g., hydrolysis of titanium tetra-iso-propoxide in $3 \mathrm{M}$ nitric acid to give $\mathrm{Ti}\left(\mathrm{OH}, 2^{2+}\right.$ ions, as indicated in the speciation diagram for $\mathrm{Ti}$ ions shown in Figure 23 [20]). Controlled precipitation of the metal oxide can be obtained by placing the solution of metal ions in a dialysis bag (molecular weight cutoff of ca. 3500) and dialyzing against distilled water. As the $\mathrm{pH}$ slowly rises in the dialysis bag (for formation of $\mathrm{TiO}_{2}$ or $\mathrm{ZrO}_{2}$ ), the solubility limit for the metal oxide will be exceeded and precipitation will begin. However, the sol is still quite acidic when precipitation starts, so the suspension is still well below its isoelectric point. Thus, the ultrafine particles that form will quickly develop a 

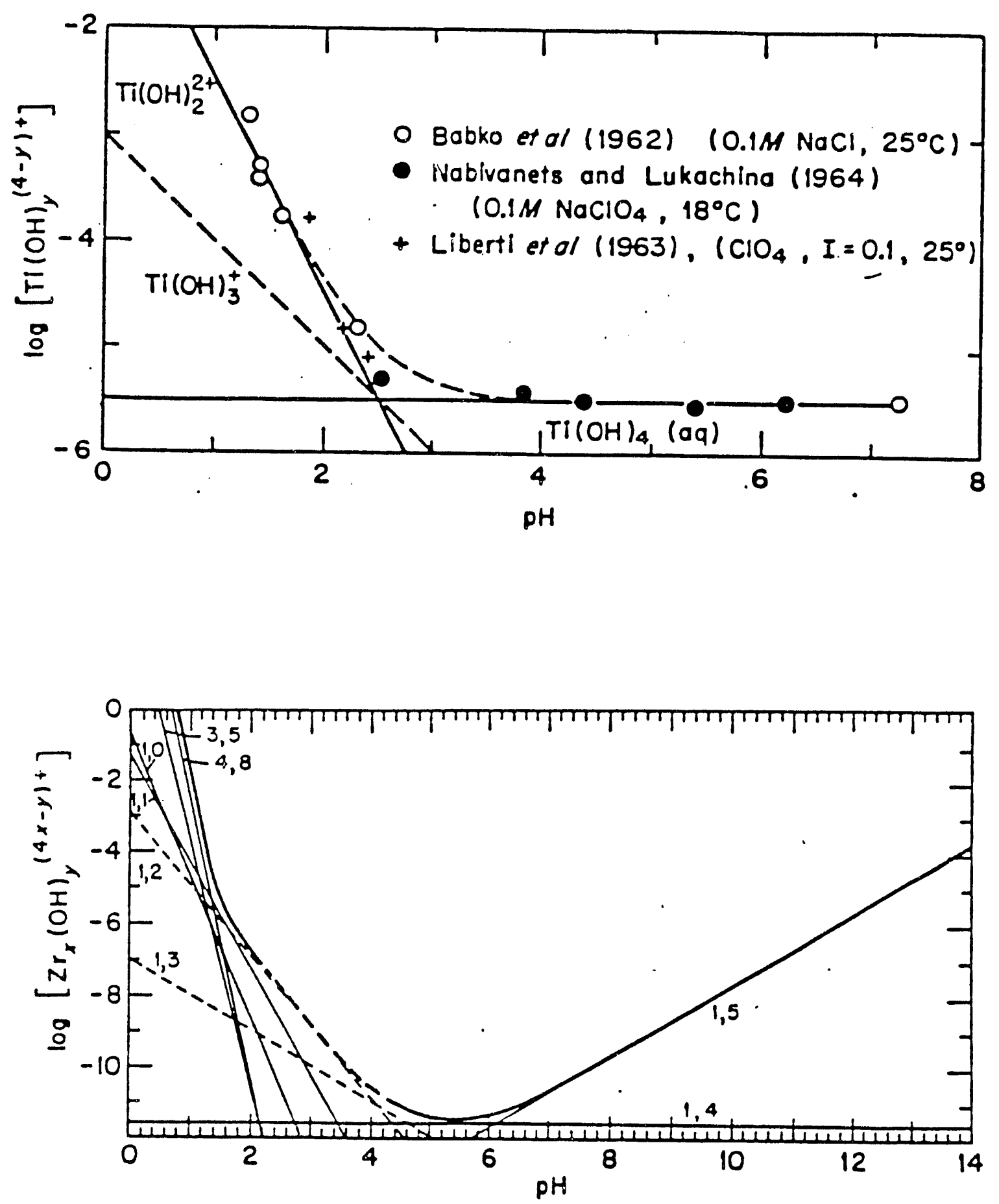

Figure 23. Speciation diagrams for $\mathrm{Ti}$ and $\mathrm{Zr}$ ions dissolved in water [20]. 
large surface charge. If this charge is sufficiently high and the ionic strength in the system is below the critical coagulation concentration for the particles, these nanoparticles (ca. $3 \mathrm{~nm}$ in diameter for $\mathrm{TiO}_{2}$ ) will form a stable sol $[18,19]$.

An outline of the general steps required to synthesize materials by the solubility control method is given in Figure 24. Note that this process is not limited to the acidic hydrolysis of relatively insoluble metal oxides. A basic hydrolysis of tetraethyl orthosilicate (TEOS), followed by dialysis against water, has been used to prepare nanoparticulate silica [21].

In order for this process to work, the $\mathrm{pH}$ and ionic strength of the sol must be carefully controlled. Two factors that affect the ionic strength of the sol should be mentioned.

(1) Metal salts could replace alkoxides in this process, as the ionic strength of the final system will be the same no matter which form of the precursor is used. The form chosen will depend primarily on convenience (e.g., titanium alkoxides are relatively stable and easily obtained from commercial sources).

(2) The ionic strength is also influenced by the loss of material through the dialysis membrane. In practice, we have found that most metal ions can be retained in the dialysis bag. Since the mobilities of metal ions are much less than that of protons, reactions occurring near the dialysis membrane will be preferentially controlled by changes in $\mathrm{pH}$ rather than by changes in the concentration of the metal ion. One result of this situation is that, during the early stages of the synthesis, a sharp pH gradient is established across the dialysis membrane. This gradient extends into the boundary layers of the solutions on both sides 


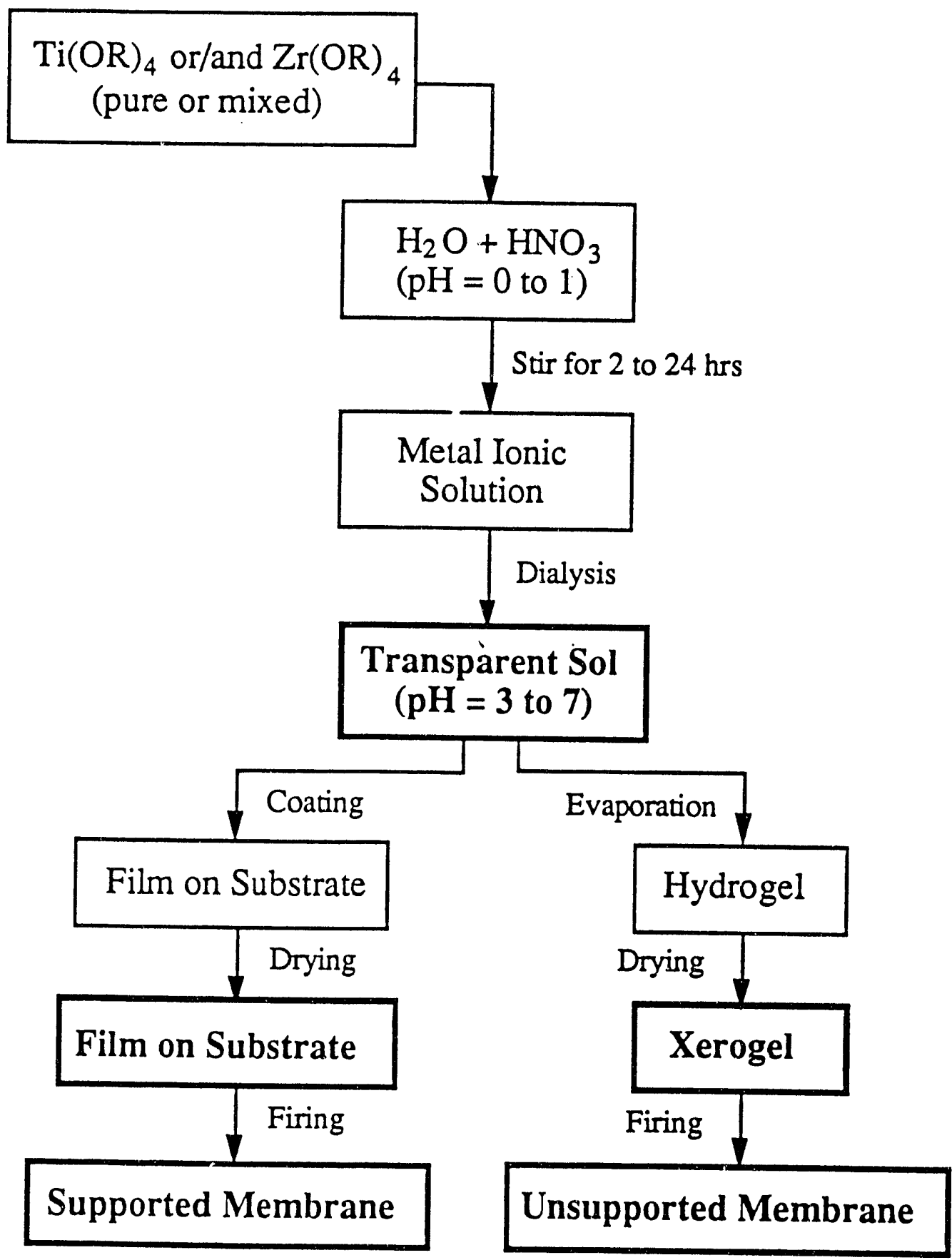

Figure 24. General procedure for preparing ceramic membranes via the solubility control method. 
of the membrane. Since the $\mathrm{pH}$ of the sol is slightly below the pH at which precipitation occurs and since the mobility of the metal ion is relatively low, one expects most of the precipitation to occur near the membrane, but within the dialysis bag. If the $\mathrm{pH}$ gradient is such that a low $\mathrm{pH}$ is established outside the dialysis bag, as could occur later in the synthesis, then precipitation would also occur outside the bag and much of the metal oxide could be lost. In order to maintain a steep $\mathrm{pH}$ gradient across the dialysis bag during the reaction, the entire system is stirred and the dialyzing water is replaced every few hours. Thus, the dialyzing water should never become acidic enough to cause precipitation of the oxide outside of the dialysis bag.

To date, the ionic strength and $\mathrm{pH}$ in these systems have been controlled well enough to demonstrate the feasibility of this synthetic scheme and to study the development of charge on the nanoparticles. Thermal stabilities of unsupported membranes prepared from these materials have also been studied. However, the parameters that define the applicable synthesis conditions have not been fully characterized. Thus, a detailed study of this synthetic approach must still be performed in order to determine:

1) how close the $\mathrm{pH}$ of the sol must be set to the $\mathrm{pH}$ at which precipitation occurs in order to limit particle growth and form nanoparticles;

2) how the final size of the nanoparticles is affected by the $\mathrm{pH}$ and ionic strength of the sol;

3) how the final size of the nanoparticles is affected by the concentration of the metal ions in the sol; and

4) how all of these factors affect the aging and growth of the nanoparticles. 


\section{b. Detailed Procedures}

A sol containing ultra-fine particles of titania was prepared by slowly dripping $8.9 \mathrm{~mL}$ of titanium tetra-iso-propoxide into strongly acidic water ( 88 $\mathrm{mL} \mathrm{H}_{2} \mathrm{O}+20 \mathrm{~mL}$ conc $\mathrm{HNO}_{3}$ ) with rapid stirring. Any precipitates formed during hydrolysis dissolved immediately. The clear solution was poured into a dialysis membrane (Spectra-Por 3, molecular weight cutoff of 3500) and dialyzed against $4 \mathrm{~L}$ of deionized water. By changing the dialysis water four times over the next $36 \mathrm{~h}$, one obtains a transparent sol with a $\mathrm{pH}$ of 3.3 and a $\mathrm{TiO}_{2}$ concentration of ca. $14.5 \mathrm{~g} / \mathrm{L}$ (assuming no loss of $\mathrm{Ti}$ from the dialysis bag). A xerogel prepared from this sol had a porosity of 498, a specific surface area of $420 \mathrm{~m}^{2} / \mathrm{g}$ and a pore size distribution that contained no mesopores. This PSD did appear to have a peak that was beginning to form at the lower limit of applicability of the technique (pores with diameters of $1.5 \mathrm{~nm}$ ). Xerogels could then be fired to obtain unsupported ceramic membranes.

The solubility control technique can also be used to prepare unsupported membranes of nanoparticulate zirconia. Addition of $11 \mathrm{~mL}$ of zirconium tetran-propoxide to acidified water ( $150 \mathrm{~mL} \mathrm{H} \mathrm{H}_{2} \mathrm{O}+9 \mathrm{~mL}$ conc $\mathrm{HNO}_{3}$ ), followed by dialysis as described above, gave a sol of ultra-fine zirconia particles. Mixed titania-zirconia membranes were also prepared by this technique. In this case, the desired amounts of titanium tetra-iso-propoxide and zirconium tetra-n-propoxide were mixed together and stirred for one hour (a total volume of ca. 10 to $11 \mathrm{~mL}$ of mixed alkoxides was used). This mixture was then added to acidified water $\left(88 \mathrm{~mL} \mathrm{H} \mathrm{H}_{2} \mathrm{O}\right.$ and $20 \mathrm{~mL}$ conc $\mathrm{HNO}_{3}$ ). The above procedure was then followed to prepare unsupported membranes of the desired composition. 


\section{c. Charge Development on Particles}

Even thugh the nanoparticulate titania and zirconia sols formed by the solubility control method are visually transparent, the ultra-fine particles in the sols still scatter laser light effectively. Thus, the charge developed on these particles can be monitored by measuring their mobilities using the technique of microelectrophoresis [19]. Figures 25 and 26 depict the mobilities of titania and zirconia particles, respectively, as functions of $\mathrm{pH}$ both after initial formation and after aging for three months. Nobility measurements were performed both in the presence of an electrolyte buffer to maintain the ionic strength constant and in the absence of added electrolyte to better simulate the behavior of a real system. In the latter case, the ionic strength changed with $\mathrm{pH}$.

Figure 25 indicates that che isoelectric points (IEP) for these titania particles were related to the nature of the particles as determined from X-ray diffraction studies but were independent of the electrolyte concentration. While the initial particles were amorphous and had an IEP at $\mathrm{pH} 4.7$, anatase crystals formed as the particles aged. Moreover, the IEP changed from 4.7 to 5.8. (Crystal size measurements based on X-ray line broadening showed the average size of the anatase particles to be $2.5 \mathrm{~nm}$.) However, the $\mathrm{ZrO}_{2}$ particles remained amorphous even after aging for 3 months. There was only a slight shift in the IEP of the $\mathrm{ZrO}_{2}$ particles (from 6.6 to 7.0 during the aging process (see Figure 26). The data presented in these figures indicate that, at $\mathrm{pH}$ values below 3 , the particles were highly charged, as expected.

Because the nobility curves of the freshly prepared titania and zirconia particles were displaced by 1-2 pH units, mobilities of mixed titania-zirconia 

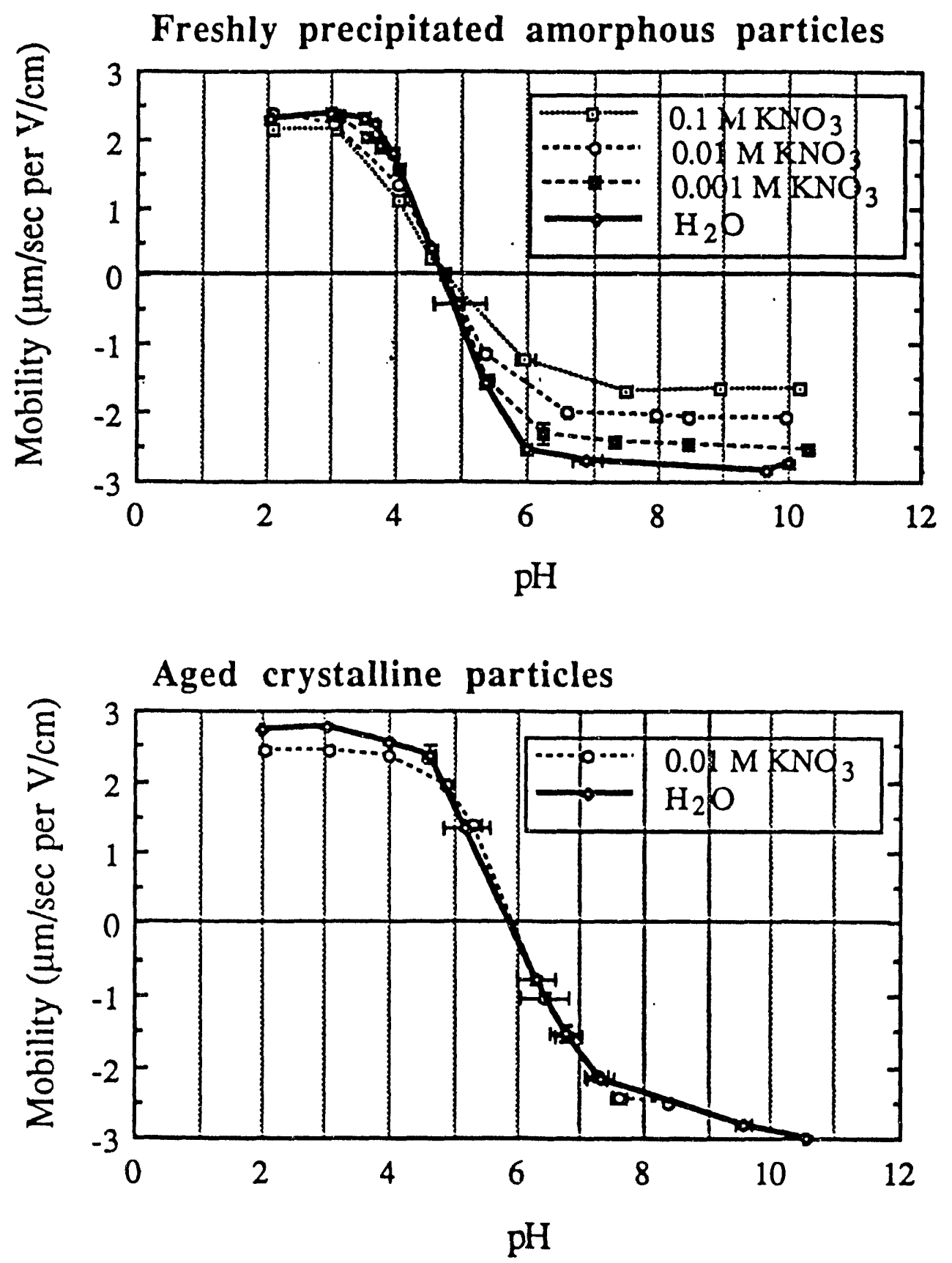

Figure 25. Mobilities of titania particles either freshly prepared or aged for three months. Samples were dispersed in either $\mathrm{KNO}_{3}$ solutions or deionized water. 

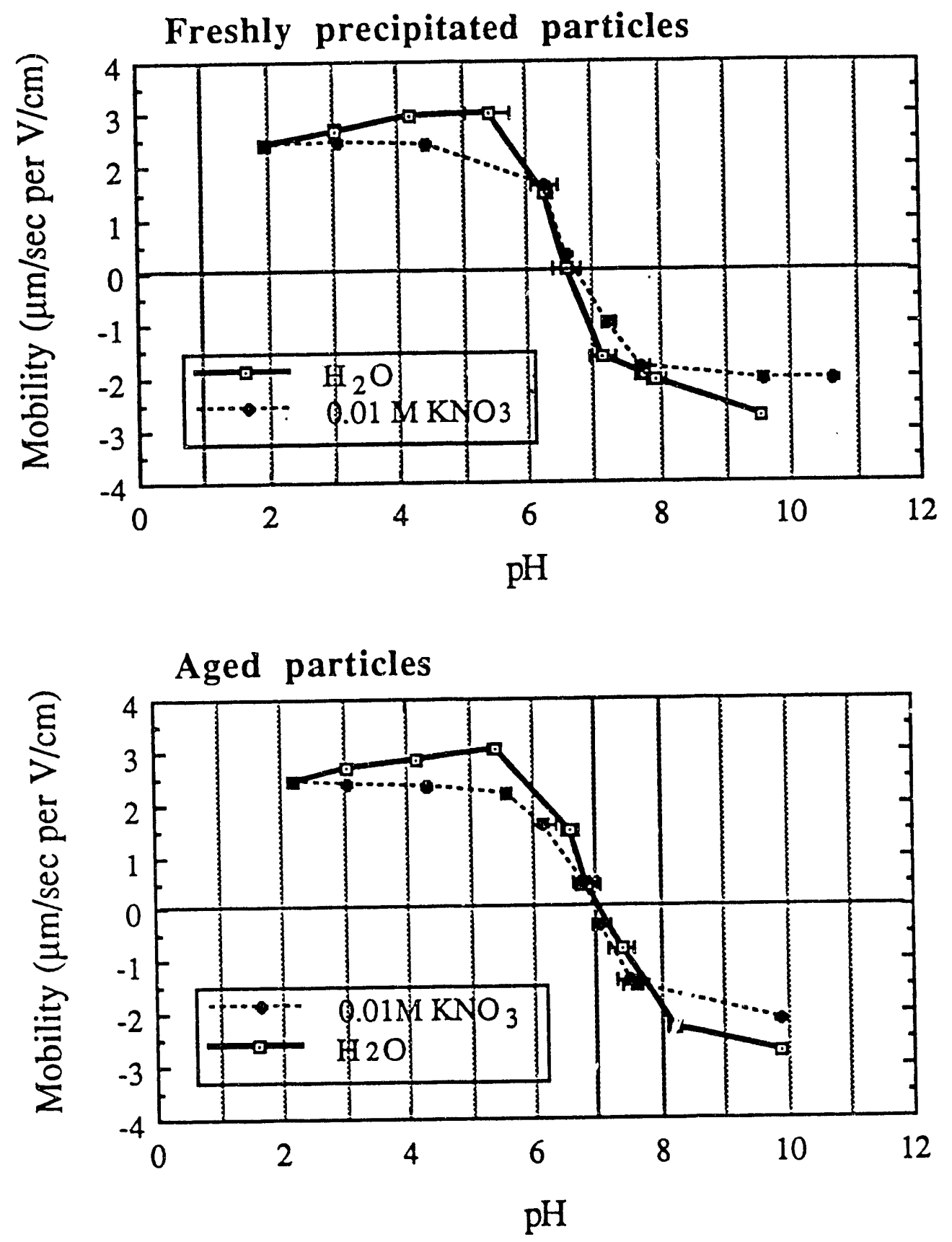

Figure 26. Mobilities of zirconia particles either freshly prepared or aged for three months. Samples were dispersed in either $\mathrm{KNO}_{3}$ solutions or deionized water. 
particles were also measured. Figure 27 depicts mobility data for pure titania and zirconia as well as $10 \% \mathrm{ZrO}_{2} \cdot 908 \mathrm{TiO}_{2}$ and $508 \mathrm{ZrO}_{2}-508 \mathrm{TiO}_{2}$ particles suspended in $0.01 \mathrm{M} \mathrm{KNO}_{3}$ solutions. Inspection of the data confirms that the IEP of an individual particle depends on its composition.

The observed variations in the IEP with the composition of the particles could be due to uniform mixing of the metal components in the mixed oxide particles or to heterocoagulation of pure $\mathrm{TiO}_{2}$ and $\mathrm{ZrO}_{2}$ particles. In the former case, one would expect the mixed oxide particles to have uniform charging properties and a narrow distribution of mobilities. In the latter case, one would expect a wider variation in the charging of the various heterocoagulated particles that would lead to a fairly wide distribution of mobilities. Since

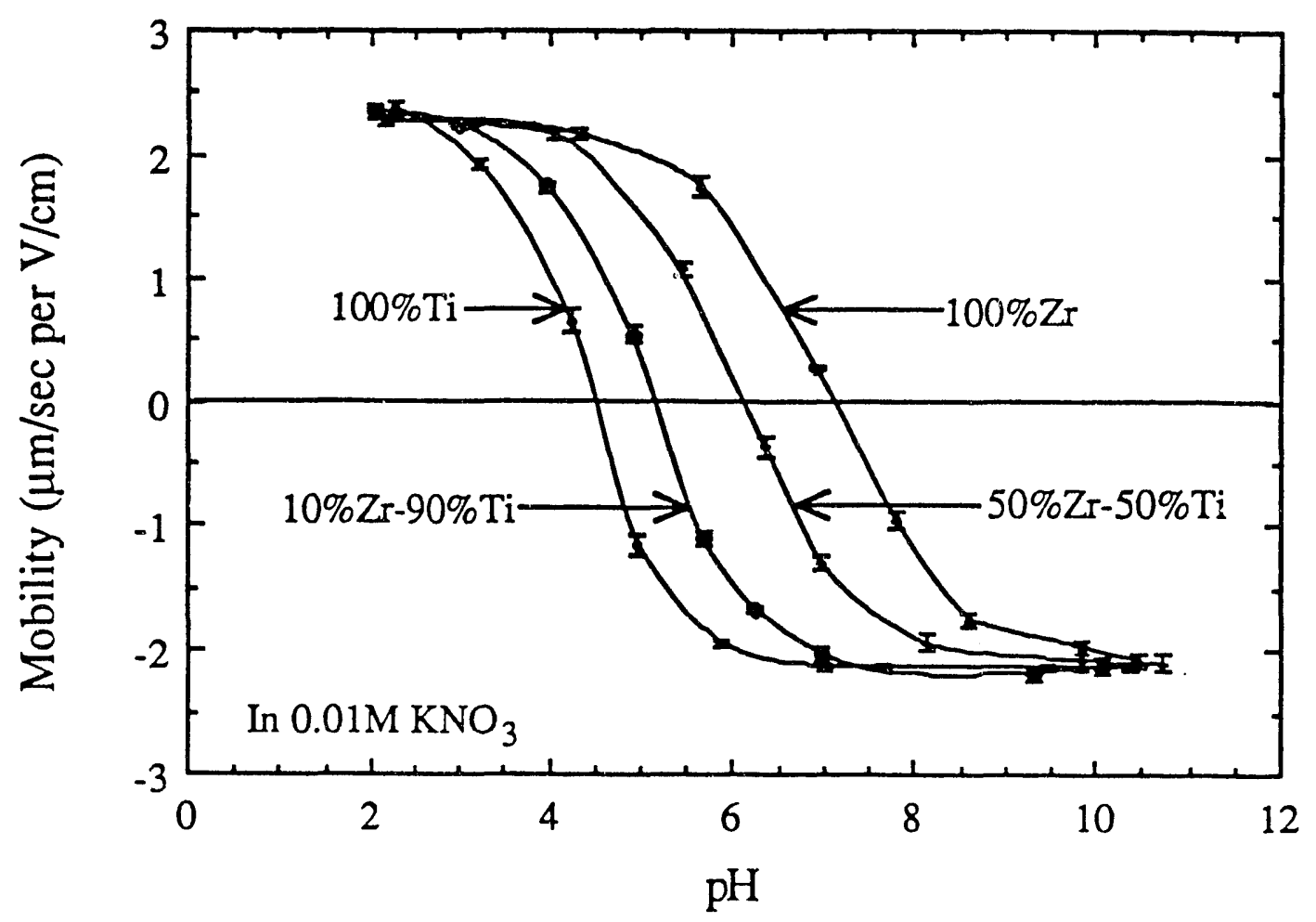

Figure 27. Mobilities of freshly prepared $\mathrm{TiO}_{2}, \mathrm{ZrO}_{2}$ and mixed $\mathrm{TiO}_{2}-\mathrm{ZrO}_{2}$ particles. Samples were dispersed in $0.01 \mathrm{M} \mathrm{KNO}_{3}$ solutions. 
the experimentally observed distributions of mobilities in the mixed oxide systems were all quite narrow, these results support the hypothesis that true mixing took place on the molecular level when the mixed oxide particles were formed. However, further experimentation is needed to confirm this hypothesis .

\section{d. Thermal Stability of Unsupported Membranes}

Ceramists have exhibited increasing interest in preparing ceramic materials from sub-micron size powders because the higher surface energies of these powders cause them to sinter and densify at much lower temperatur.s than conventional powders. U1tra-fine particle powders should densify at even lower temperatures than those observed for sub-micron powders of a given material. When the thermal stabilities of unsupported titania membranes prepared from conventional sols and nanoparticle sols were compared, it was found that the membranes prepared from conventional sols were indeed more stable.

A conventional $\mathrm{TiO}_{2}$ sol was prepared by peptization, then dialyzed to $\mathrm{pH}$ 3.5. This process gave a sol that contained anatase particles with diameters of 5-6 nm. A TiO 2 sol was also prepared by solubility control to $\mathrm{pH} 3.3$. This protocol led to a sol that contained anatase particles with diameters of 2-3 nm. Xerogels prepared from these two sols were fired under the same conditions, and the properties of the resulting ceramic membranes were compared. Figure 28 contains plots of the measured porosities of these membranes versus firing temperature. The data clearly indicate the loss of thermal stability that is associated with nanoparticle membranes. Membranes formed from pure nanoparticulate zirconia sols also exhibited poor thermal stability. 


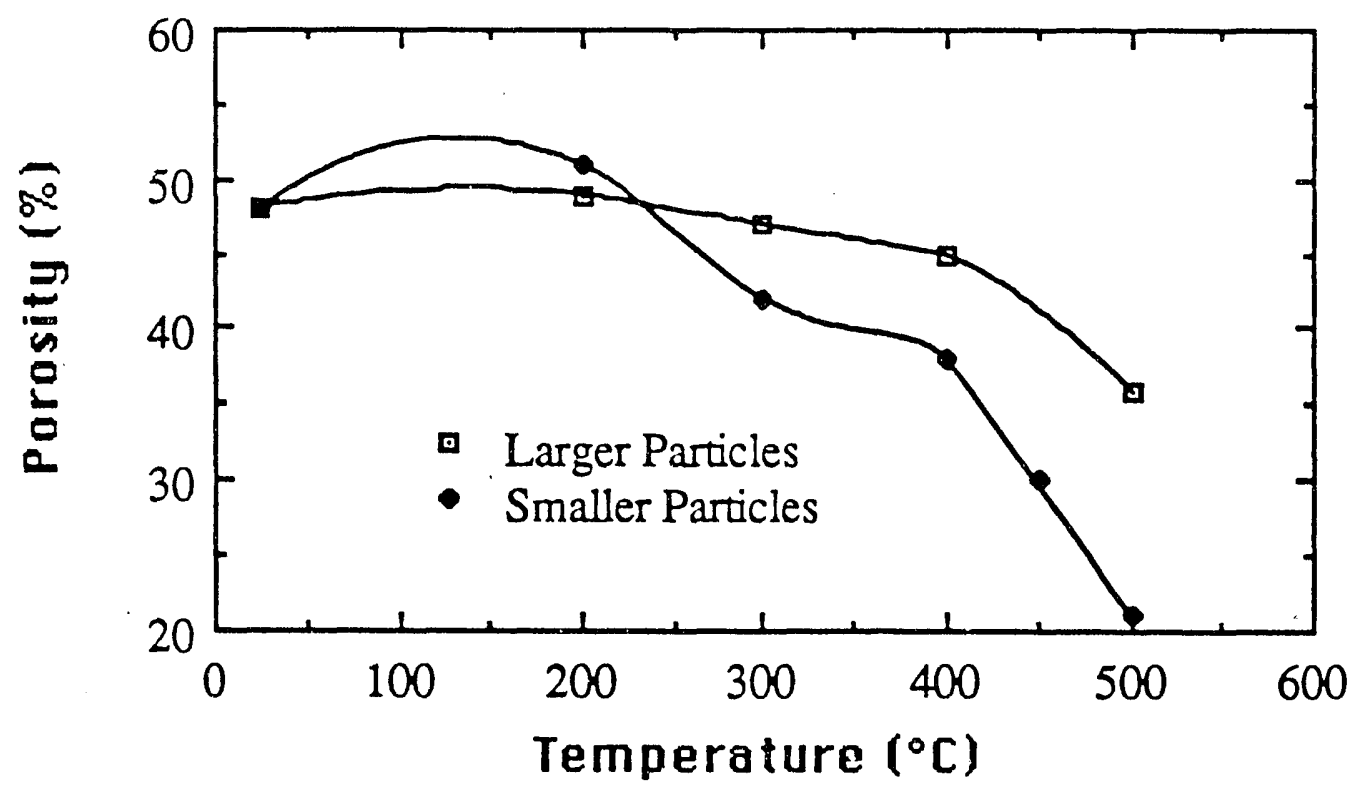

Figure 28. Porosities of titania membranes composed of particles with different sizes as a function of the firing temperature. All membranes were fired at $2^{\circ} \mathrm{C}$ 'min to the desired temperature and held at this temperature for $30 \mathrm{~min}$.

X-ray diffraction studies performed on unsupported membranes prepared from nanoparticulate $\mathrm{ZrO}_{2}$ sols indicate that the poor thermal stability of these membranes may be associated with changes in crystallinity. The diffraction patterns shown in Figure 19 were obtained from a membrane prepared from a conventional peptized sol. They indicate that monoclinic $\mathrm{ZrO}_{2}$ was beginning to form when the membrane in question was fired to $400^{\circ} \mathrm{C}$. Contrast these patterns with those in Figure 29, which shows the X-ray diffraction patterns obtained for nanoparticle membranes formed from a $\mathrm{ZrO}_{2}$ sol. For the nanoparticle membranes, well-defined crystallinity was obtained by firing to $400^{\circ} \mathrm{C}$. Evidence for the presence of both the monoclinic and tetragonal phases of zirconia in membranes fired to only $400^{\circ} \mathrm{C}$ was surprising. 


\section{$\mathrm{ZrO}_{2}$}

\section{Xerogel}

\section{$200^{\circ} \mathrm{C}$}

\section{$300^{\circ} \mathrm{C}$}

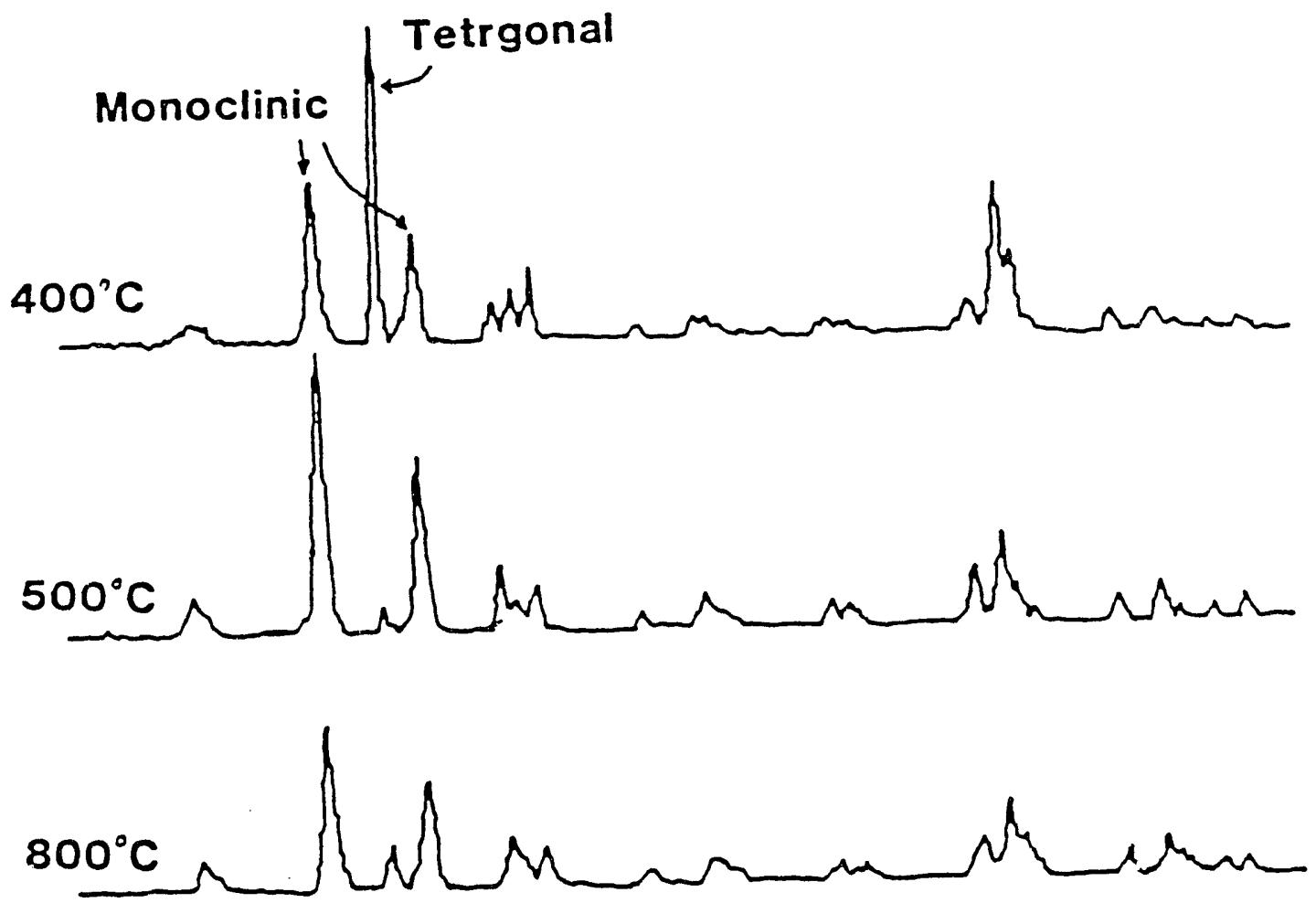

Figure 29. X-Ray diffraction patterns of zirconia membranes heated to the indicated temperature at $2^{\circ} \mathrm{C} / \mathrm{min}$ and held at this temperature for one hour. The precursor sols were prepared by the solubility control method. 
The limited thermal stabilities of membranes prepared from nanoparticulate sols were of great concern because this property could limit the eventual commercial applications of these materials. As discussed in Section IV.A.4.b, the thermal stabilities of mixed oxide membranes prepared from conventional sols were significantly improved over the thermal stabilities of single component oxide membranes prepared from similar materials. Consequently, the properties of a $108 \mathrm{ZrO}_{2}-908 \mathrm{TiO}_{2}$ nanoparticle membrane fired at different temperatures were compared to the properties of a pure $\mathrm{TiO}_{2}$ nanoparticle membrane fired under identical conditions. As shown in Figures 30 through 32, the mixed oxide membrane exhibited a large improvement in thermal stability.

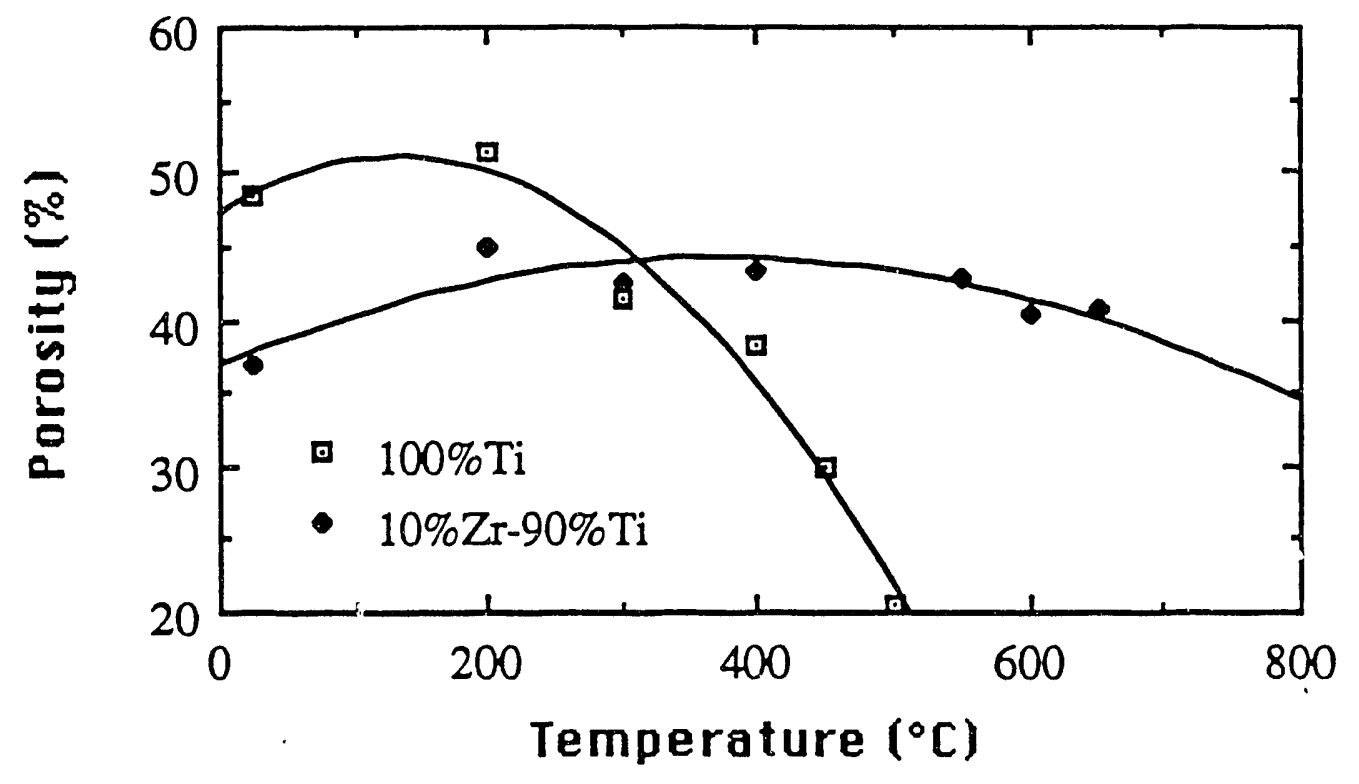

Figure 30. Porosities of pure $\mathrm{TiO}_{2}$ and $108 \mathrm{ZrO}_{2}-908 \mathrm{TiO}_{2}$ nanoparticle membranes prepared from sols made by the solubility control method. All membranes were heated at $2^{\circ} \mathrm{C} / \mathrm{min}$ to the indicated firing temperature and held at this temperature for $30 \mathrm{~min}$. 


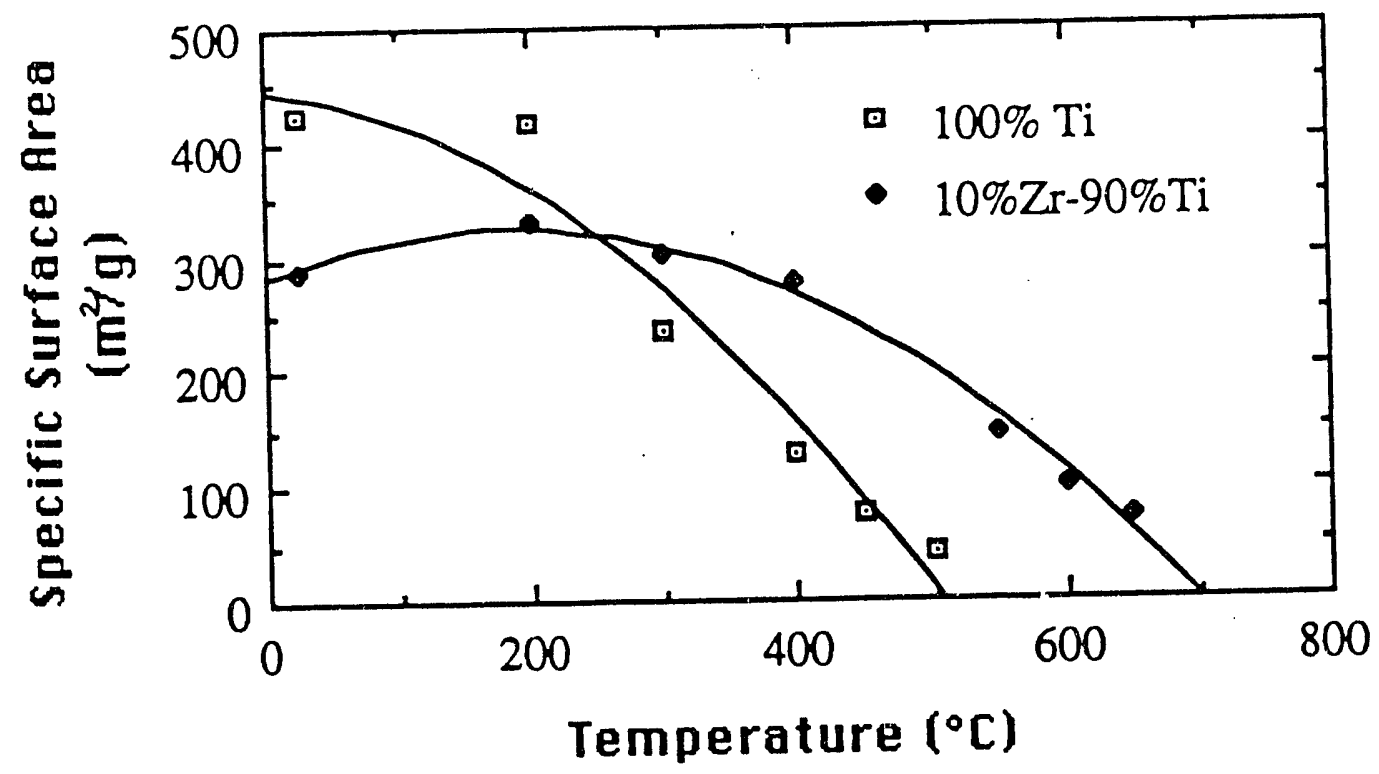

Figure 31. Specific surface areas of pure $\mathrm{TiO}_{2}$ and $108 \mathrm{ZrO}_{2}-908 \mathrm{TiO}_{2}$ nanoparticle membranes prepared from sols made by the solubility control method. All membranes were heated at $2^{\circ} \mathrm{C} / \mathrm{min}$ to the indicated firing temperature and held at this temperature for $30 \mathrm{~min}$.

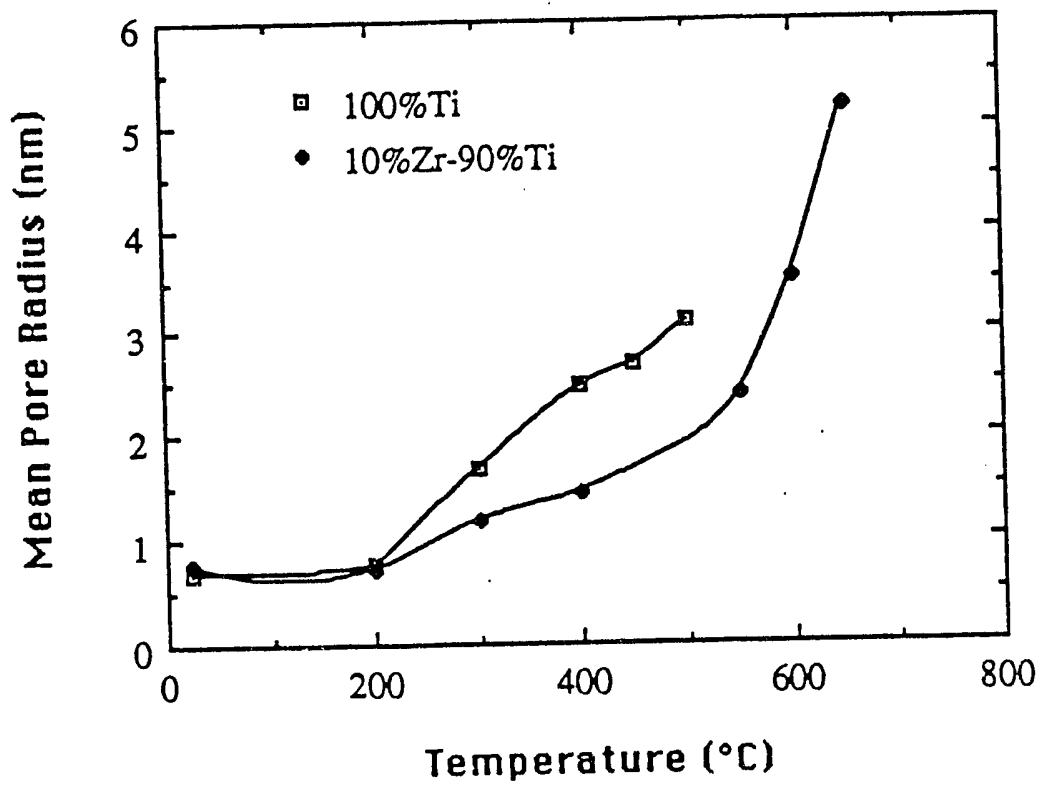

Figure 32. Pore sizes of pure $\mathrm{TiO}_{2}$ and $108 \mathrm{ZrO}_{2}-908 \mathrm{TiO}_{2}$ nanoparticle membranes prepared from sols made by the solubility control method. All membranes were heated at $2^{\circ} \mathrm{C} / \mathrm{min}$ to the indicated firing temperature and held at this temperature for $30 \mathrm{~min}$. 


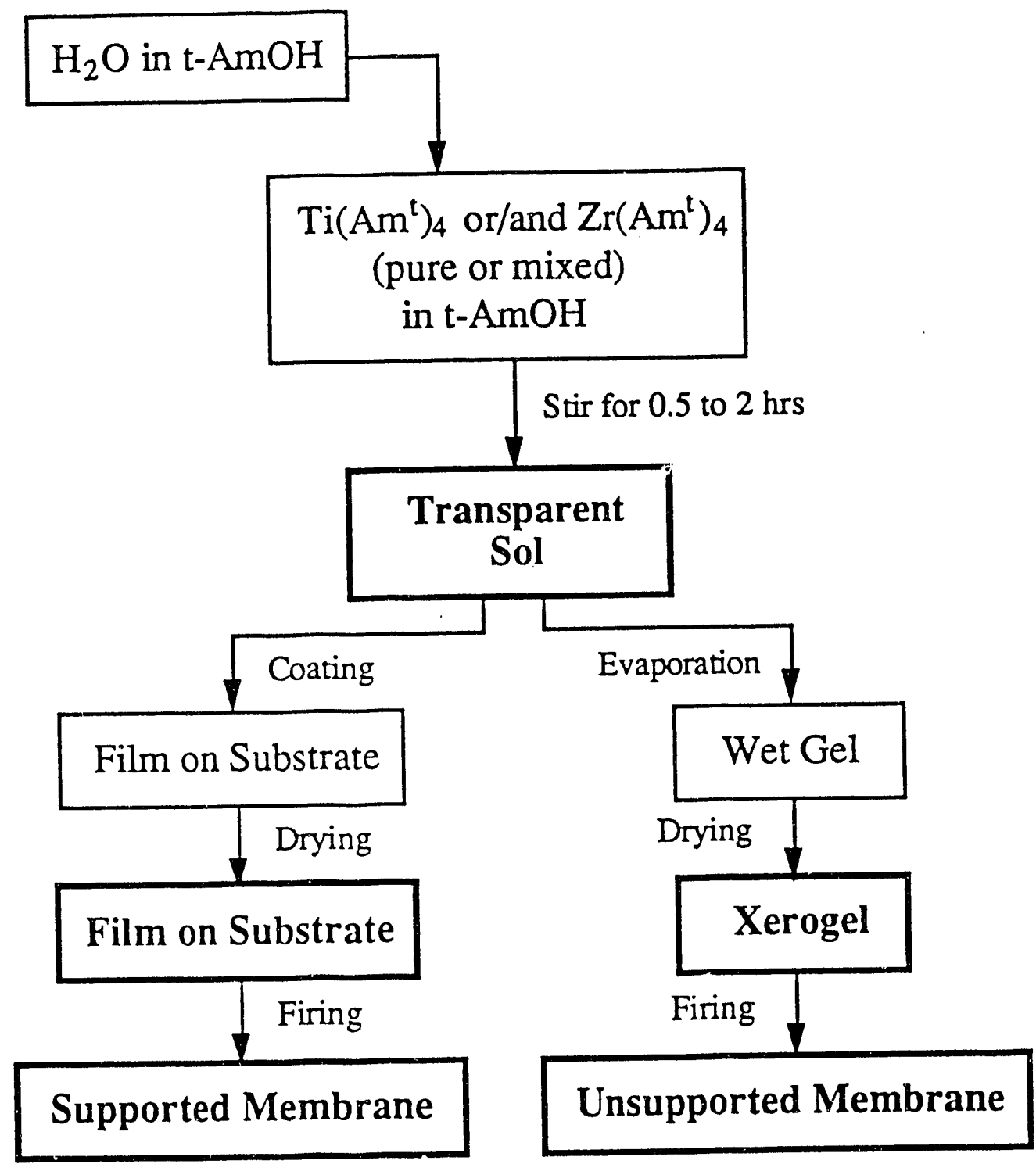

Figure 33. General procedure for preparinr ceramic membranes via the steric hindrance method. 
(For example, $30 \mathrm{~mL}$ of titanium tetra-1so-propoxide and $56.5 \mathrm{~mL}$ of tert-amyl alcohol would be added to $240 \mathrm{~mL}$ of benzene.) The alcohol exchange reaction is driven to completion by distilling off the azeotrope that forms between the alcohol from the commercial alkoxide and benzene (at $71.4^{\circ} \mathrm{C}$ for benzene and iso-propano1). This process is feasible because the alcohols from the commercially available alkoxides will all form azeotropes with benzene that have lower boiling points than the azeotrope formed from benzene and tert-amyl alcohol, which boils above $100^{\circ} \mathrm{C}$. NMR spectra of the resulting products indicated that, in both the $\mathrm{Ti}$ and $\mathrm{Z}$.r systems, this procedure led to replacement of over 908 of the original alkoxide groups by tert-amyl alkoxide groups. Sols were obtained by first dissolving both the freshly prepared tertamyl alkoxide solution and water in separate but equal amounts of tert-amyl alcohol. (The water was usually acidified with enough $\mathrm{HNO}_{3}$ to give the desired $\mathrm{pH}$, typically $\mathrm{pH} 2$.) The two tert-amyl alcohol solutions were then mixed by dripping the solution containing water into the solution of the alkoxide. This process gave a transparent solution that was aged for $2 \mathrm{~h}$ (with stirring) and then placed in polystyrene dishes for gelation. Evaporation was carried out in desiccator boxes. This process was enhanced by using $5 \AA$ molecular sieves, which frequently had to be regenerated before the evaporation step was completed. Cracking of the resultant unsupported membranes could be minimized by carrying out the drying process over a period of several weeks.

A $208 \mathrm{ZrO}_{2}-808 \mathrm{TiO}_{2}$ nanoparticle sol was prepared as follows. $1.4 \mathrm{~mL}$ of a $1.1 \mathrm{M}$ zirconium tetra-tert-amyloxide solution in tert-amyl alcohol (as prepared by the alcohol exchange method) and $1.76 \mathrm{~g}$ of titanium tetra-tertamyloxide were dissolved in $12.8 \mathrm{~mL}$ of tert-amyl alcohol. The resulting mixed alkoxide solution was stirred for one hour. Separately, $250 \mu \mathrm{L}$ of acidified 
water at $\mathrm{pH} 2$ was added to $15 \mathrm{~mL}$ of tert-amyl alcohol. The aqueous solution was then dripped into the mixed alkoxide solution to give a transparent sol with a total metal ion concentration of $0.23 \mathrm{M}$ and a water concentration of $0.46 \mathrm{M}$. After further stirring for $2 \mathrm{~h}$, the sol was poured into polystyrene dishes to prepare xerogels [9].

\section{b. Effects of Reactant Concentration on Particle Growth}

In the solubility control approach to synthesizing nanoparticles, the overall growth of particles is minimized by the charge that the particles develop once they form. While the particles may still grow by Ostwald ripening, this process must be very slow since the sols remain transparent for several months. The steric hindrance approach to synthesizing nanoparticles is not based on controlling the electrical charge borne by the particles. Instead, particle growth is inhibited due to geometric constraints imposed by the bulky substituent groups of the precursor alkoxide. These constraints lead to such slow rates of hydrolysis and condensation that particle growth is minimized. Again, sols can be prepared that remain transparent for several months, if the sols are stored under conditions that limit contact with moisture.

Studies were also performed to determine the effects of changing the reactant concentrations on the kinetics of particle growth under conditions in which steric hindrance constraints are operative. Particle sizes were estimated from quasi-elastic light scattering measurements, and the onset of precipitation was determined visually by the appearance of cloudiness in the sol.

The data in Table XVI indicate the effects of varying the reactant concentrations on the time needed for precipitates to be observed in the sols and 
on the appearance of the xerogels prepared from these sols shortly after hydrolysis. Note that the time needed for precipitation, which indicates the integrated effect of the overall reaction rate, was dependent on the concentrations of all reactants. For a given concentration of titanium tetra-tertamyloxide ( $\mathrm{Ti}$ ), raising the concentration of water caused faster hydrolysis and faster precipitation. For a given concentration of water, raising the $\mathrm{Ti}$ concentration (thus lowering the $\mathrm{H}_{2} \mathrm{O} / \mathrm{Ti}$ mole ratio) caused slower precipitation. Both results indicate that the overall rate of reaction can be control. led by adjusting the concentration of water in the system. It was somewhat surprising to find that the rate of reaction could be innibited by conducting the reaction under more acidic conditions. It would appear that, even in these primarily alcoholic systems, enough water is present to allow proton adsorption to occur on these particles, thus causing them to be electrostatically stabilized.

Table XVI. Effects of reactant concentrations on hydrolyses performed using the steric hindrance approach.

\begin{tabular}{cccccc}
$\begin{array}{c}{[\mathrm{Ti} \text { alkoxide] }} \\
(\mathrm{M})\end{array}$ & $\begin{array}{c}{\left[\mathrm{H}_{2} \mathrm{O}\right]} \\
(\mathrm{M})\end{array}$ & $\mathrm{pH}$ & \multicolumn{2}{c}{$\begin{array}{c}\text { Precipitation Time }(\mathrm{h}) \\
\text { Room Temp. }\end{array}$} & $\begin{array}{c}\text { Appearance } \\
\text { of Xerogel }\end{array}$ \\
\hline 0.2 & 0.2 & 2.0 & No precipitation until 67 days & Clear \\
0.2 & 0.6 & 2.0 & No precipitation until 67 days & Clear \\
0.2 & 0.8 & 2.0 & 528 & 100 & Clear \\
0.2 & 1.0 & 2.0 & 216 & 15 & Clear \\
0.2 & 1.6 & 2.0 & Immediately & Immediately & White \\
& & & & & \\
0.4 & 0.8 & 2.0 & No precipitation until 67 days & Clear \\
0.4 & 1.6 & 2.0 & 1.5 & Immediately & Opaque Chunk \\
& & & & & \\
0.2 & 0.8 & 1.0 & 720 & 128 & Clear \\
0.2 & 0.8 & 2.0 & 528 & 100 & Clear \\
0.2 & 0.8 & 3.0 & 480 & 48 & Clear \\
0.2 & 0.8 & $6-7$ & 432 & 45 & Translucent
\end{tabular}


The data in Figures 34 and 35 depict the effect of using different reactant concentrations on the growth rate of the particles. In all cases, there was an induction period of at least two months during which the particles grew very slowly. During this period, the average growth rate was dependent on the molar ratio of water to alkoxide (see Figure 36). The linear relation between particle growth rate and the molar xatio of water to alkoxide, up to a molar ratio of 5 , suggests that, during the induction period, hydrolysis might be the rate limiting step governing the rate at which the particles can grow. Light scattering from the particles was relatively strong even at the time the first data point for Figures 34 and 35 was obtained. This observation suggests that particle nucleation occurred over a short period of time early in the synthesis when the concentration of hydrolyzed monomers was supersaturated. Immediately after nucleation, the concentration of monomers

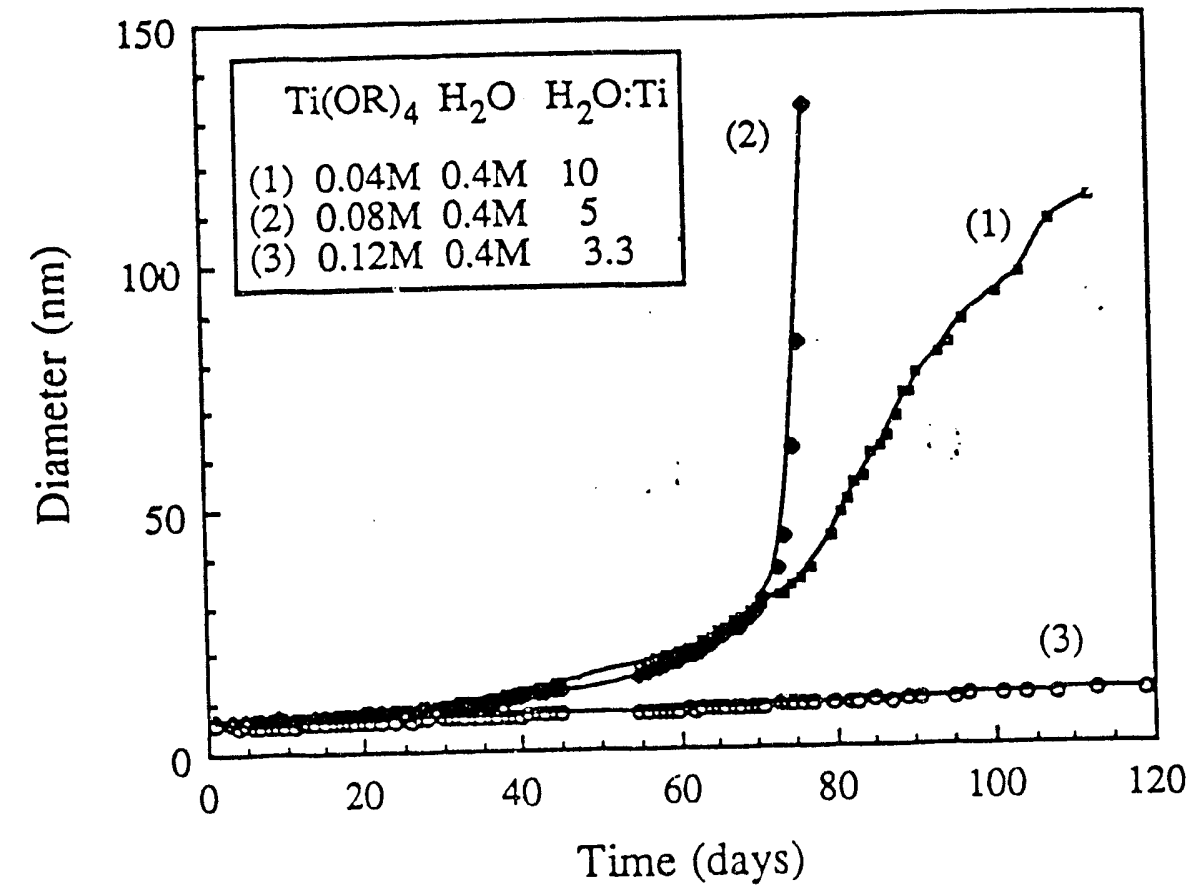

Figure 34. Change in particle size with time as a function of $\mathrm{Ti}$ alkoxide concentration. 


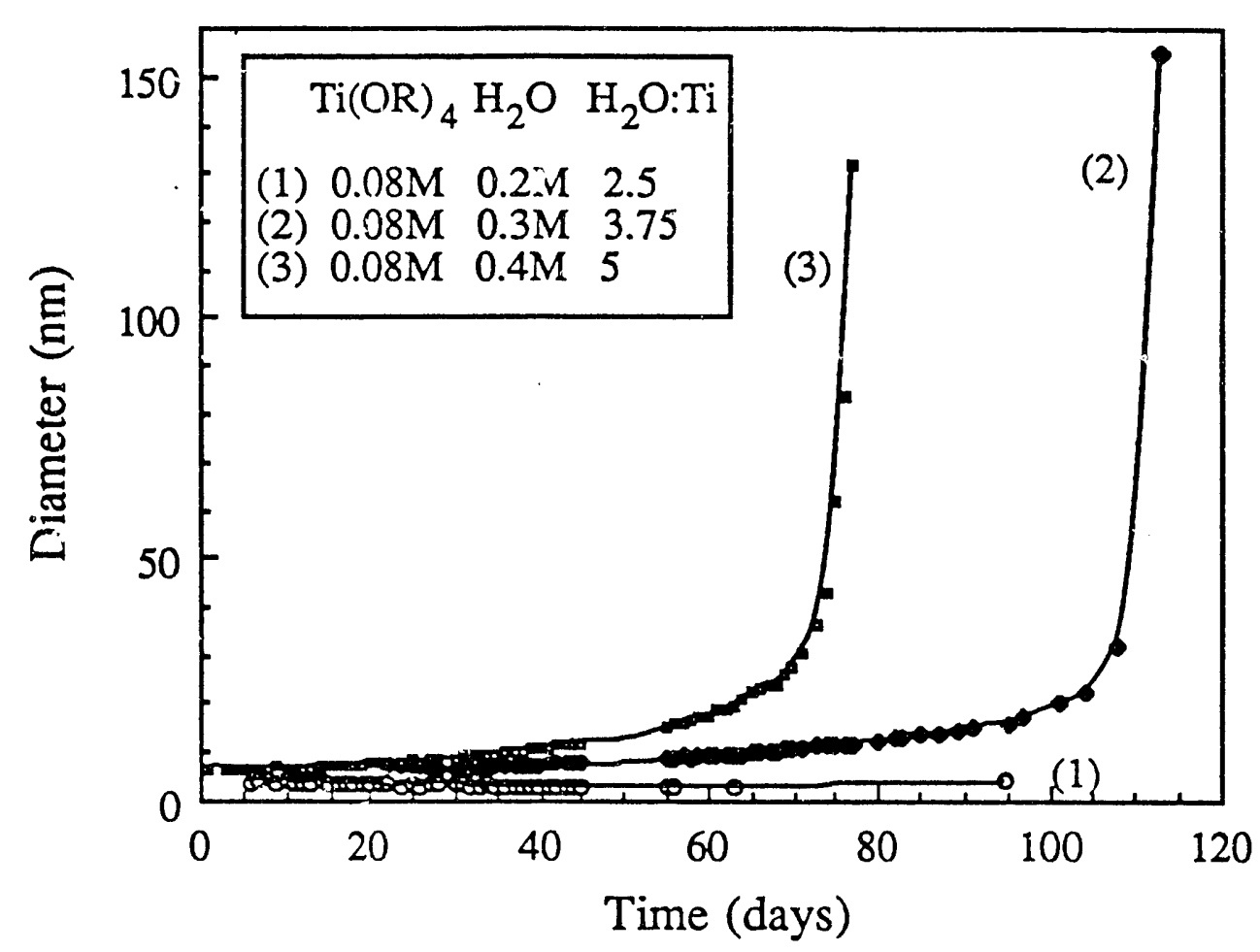

Figure 35. Change in particle size with time as a function of the concentration of water. Note that curve 2 in Figure 34 and curve 3 in Figure 35 are identical.

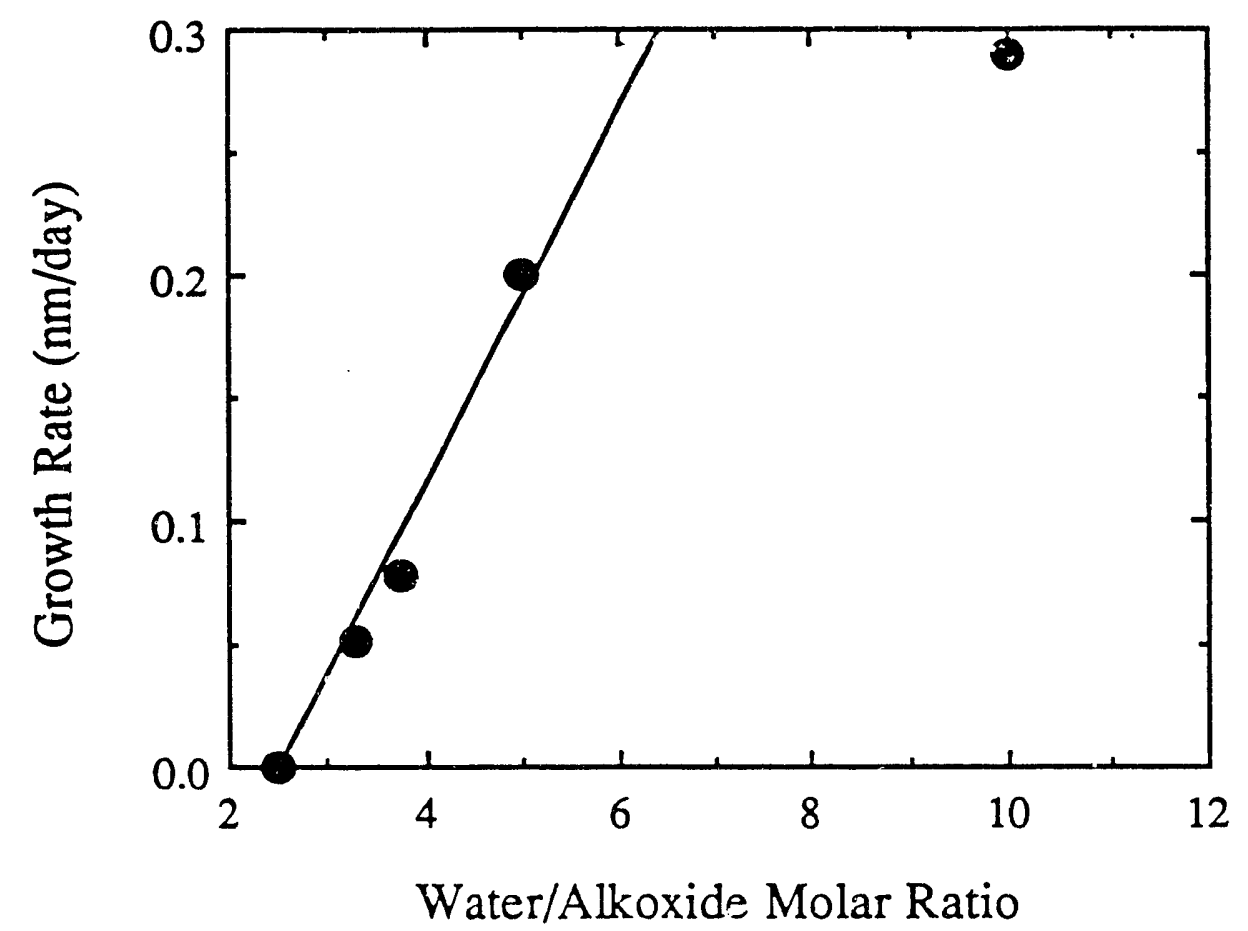

Figure 36. The effect of the molar ratio of water to alkoxide on the growth rate of particles during the induction period of particle growth. 
would drop well below the critical saturation point, so that the number of nuclei would remain relatively constant during the induction period. Since further production of monomers during this stage would be slow, additional nucleation would be inhibited. Instead, monomeric species would be incorporated into the growing particles. This model of particle growth, the MonomerAddition Growth Model, has been described in detail elsewhere [23].

Once the induction period ends, particle size increases much more rapidly. This result indicates that growth was occurring by the process of particle-particle aggregation. Average aggregation rates, as indicated by the slopes of the curves in Figures 34 and 35 , depended on the initial concentration of alkoxide in the system. After the induction period, particle growth occurred much faster in systems containing $0.08 \mathrm{M}$ alkoxide than in the system containing $0.04 \mathrm{M}$ alkoxide. However, a detailed study of aggregation in these systems, similar to that carried out for titania sols (see Section IV.A.3.d), was not pursued.

c. Thermal Stability of Unsupported Membranes

\section{Single Phase Membranes}

Alcoholic sols prepared by the steric hindrance method were evaporated to form xerogels. These xerogels, in turn, were fired at $2{ }^{\circ} \mathrm{C} / \mathrm{min}$ to various temperatures and held at the ultimate temperatures for $30 \mathrm{~min}$ to form unsupported membranes. The pore structures of these membranes were characterized by $\mathrm{N}_{2}$ sorption measurements. X-ray diffraction studies were also performed to determine the crystalline phases present in these membranes. 
Titania particles synthesized by the steric hindrance method were amorphous, unlike particles produced by solubility control and peptization in aqueous systems. The latter particles often contained anatase crystals. Membranes fabricated from sols that had been produced by the steric hindrance method gave anatase crystals when fired to $350^{\circ} \mathrm{C}$ (see Figure 37). Separate measurements of the pore structures of these membranes (see Figure 38) suggested that the onset of crystallinity was associated with the ordering of small

\section{RT}

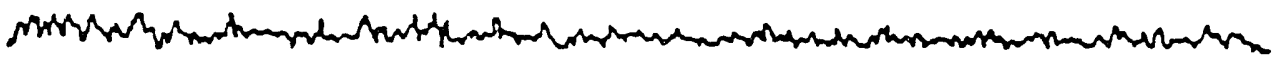

\section{$300^{\circ} \mathrm{C}$}
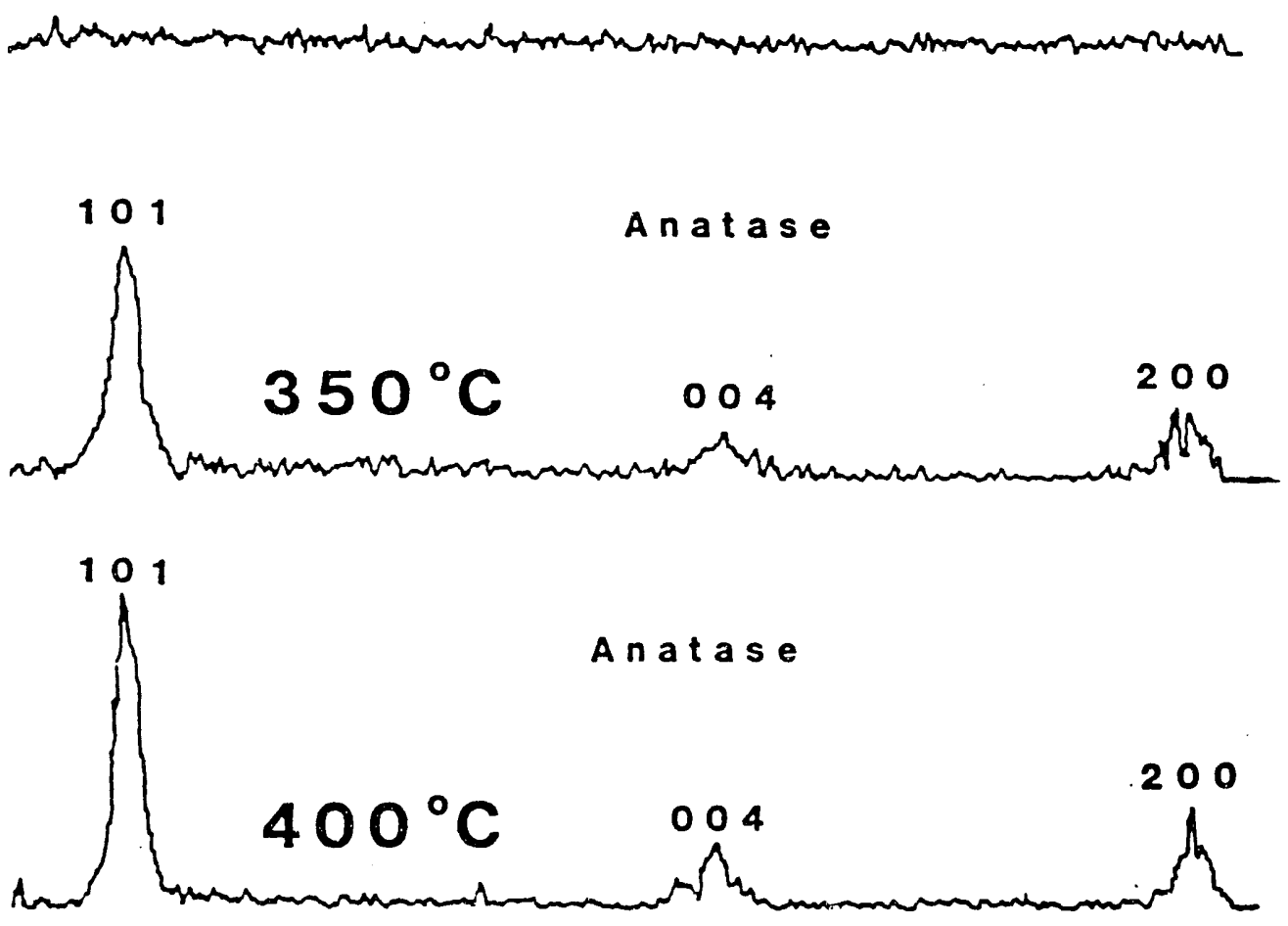

Figure 37. X-ray diffraction patterns for titania membranes fabricated from sols prepared by the steric hindrance method. All membranes were heated at $2{ }^{\circ} \mathrm{C} / \mathrm{min}$ to the indicated temperature and held at this temperature for $30 \mathrm{~min}$. 


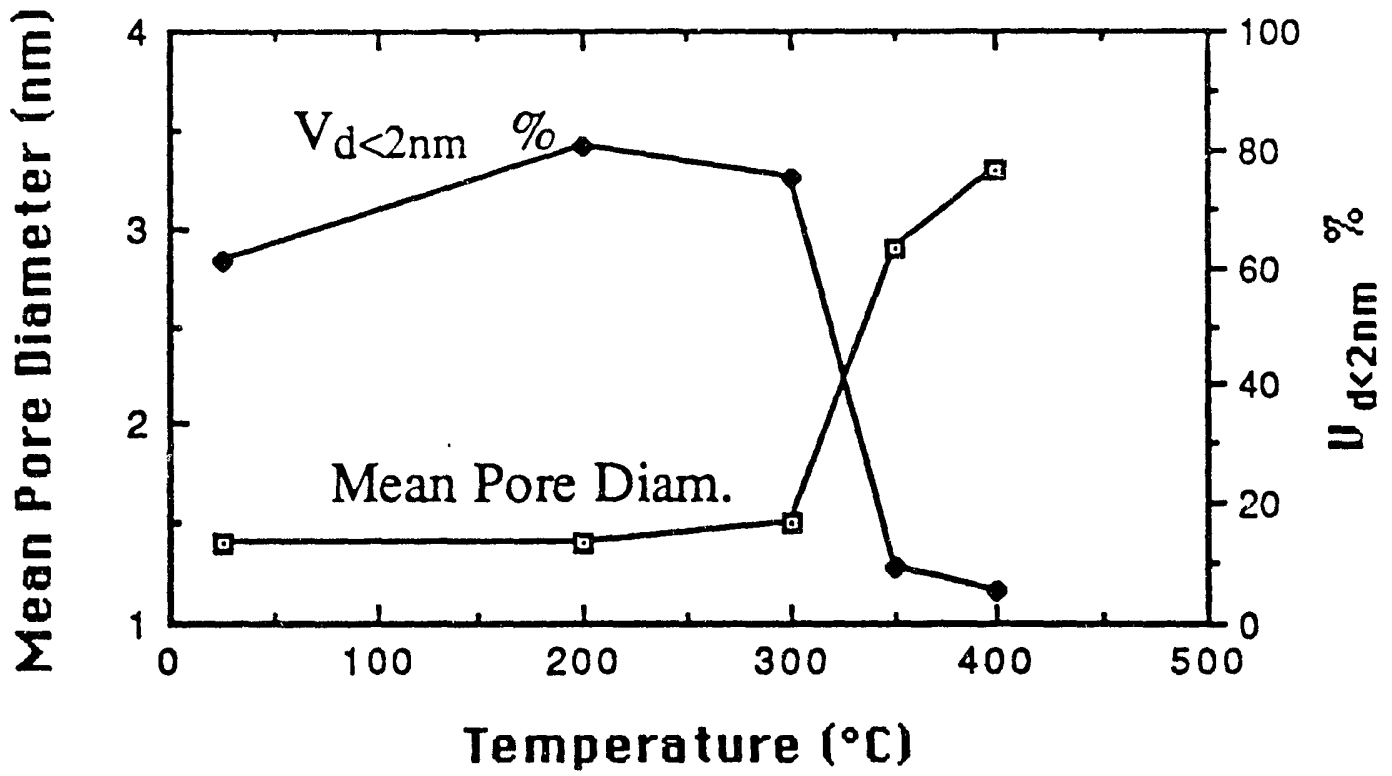

Figure 38. The effects of firing temperature on the mean pore diameter and microporosity of titania membranes. All membranes were heated at $2{ }^{\circ} \mathrm{C} / \mathrm{min}$ to the indicated temperature and held at this temperature for $30 \mathrm{~min}$.

amorphous particles into larger crystalline grains. The mean pore diameter increased markedly as the firing temperature was increased from 300 to $350^{\circ} \mathrm{C}$. The associated porosities decreased from 438 to 278 (and to 168 at $400^{\circ} \mathrm{C}$ ). The percentage of the pore volume associated with micropores with diameters below $2 \mathrm{~nm}$ (noted as $\mathrm{V}_{\mathrm{d}<2 \mathrm{~nm}}$ in Figure 38) also decreased as crystallization occurred. X-ray diffraction studiss were not performed at firing temperatures above $400^{\circ} \mathrm{C}$ because the membranes appeared to densify under these conditions. Studies performed on zirconia membranes fabricated from sols that were prepared by the steric hindrance method gave results similar to those obtained for the studies on titania membranes. As shown in Figure 39, pore diameters of these $\mathrm{ZrO}_{2}$ membranes increased dramatically as the firing temperature was raised from 300 to $350^{\circ} \mathrm{C}$. This increase in pore diameter was associated with a decrease in porosity from $30 \%$ to $18 \%$. However, this sudden change in the 
properties of the membranes did not occur while the membranes were crystallizing. Figure 40 contains $\mathrm{X}$-ray diffraction patterns obtained for $\mathrm{ZrO}_{2}$ membranes fired to different temperatures. The membrane fired to $350^{\circ} \mathrm{C}$ was still composed of amorphous particles. Tetragonal crystals of $\mathrm{ZrO}_{2}$ did not form in these membranes until a firing temperature between 350 and $400^{\circ} \mathrm{C}$ was reached.

It is interesting to compare the diffraction patterns in Figures 19, 29 and 40 which indicate the onset of crystallization in $\mathrm{ZrO}_{2}$ membranes synthesized by the peptization, solubility control and steric hindrance methods, respectively. While crystallization always began at temperatures below $400^{\circ} \mathrm{C}$, the initial crystalline form varied depending on the method used to synthesize the sols. Peptization gave monoclinic crystals, steric hindrance resulted in tetragonal crystals, and solubility control produced a mixture of both types of crystals. This behavior probably reflects the extremely small sizes of the

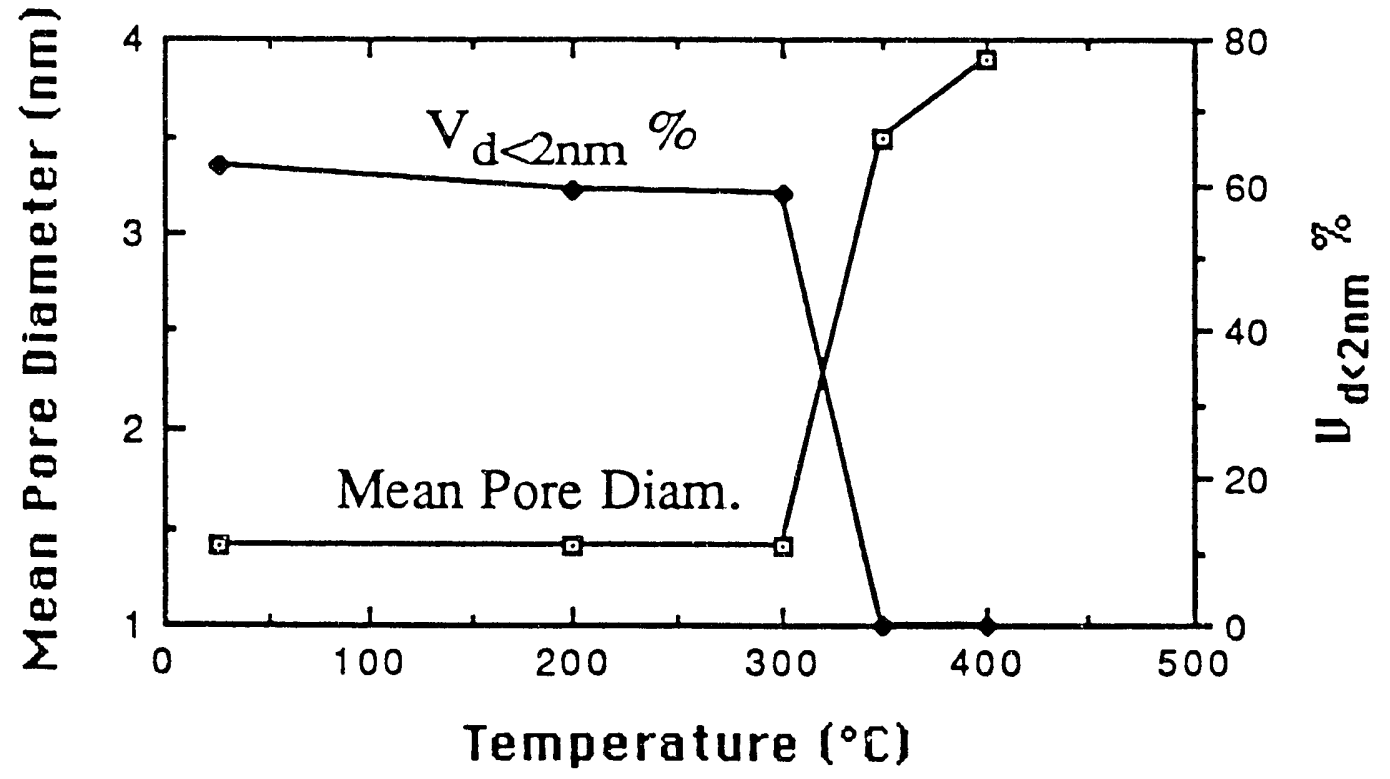

Figure 39. The effects of firing temperature on the mean pore diameter and microporosity of zirconia membranes. All membranes were heated at $2^{\circ} \mathrm{C} / \mathrm{min}$ to the indicated temperature and held at this temperature for $30 \mathrm{~min}$. 


\section{R T}

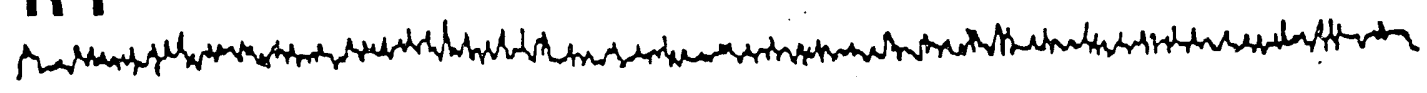

\section{$350{ }^{\circ} \mathrm{C}$}

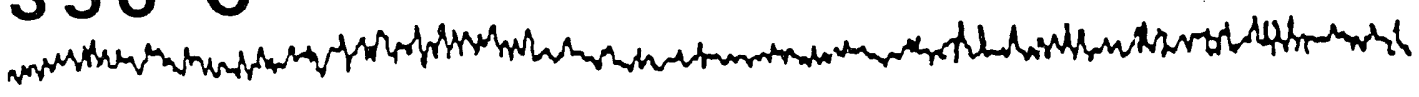
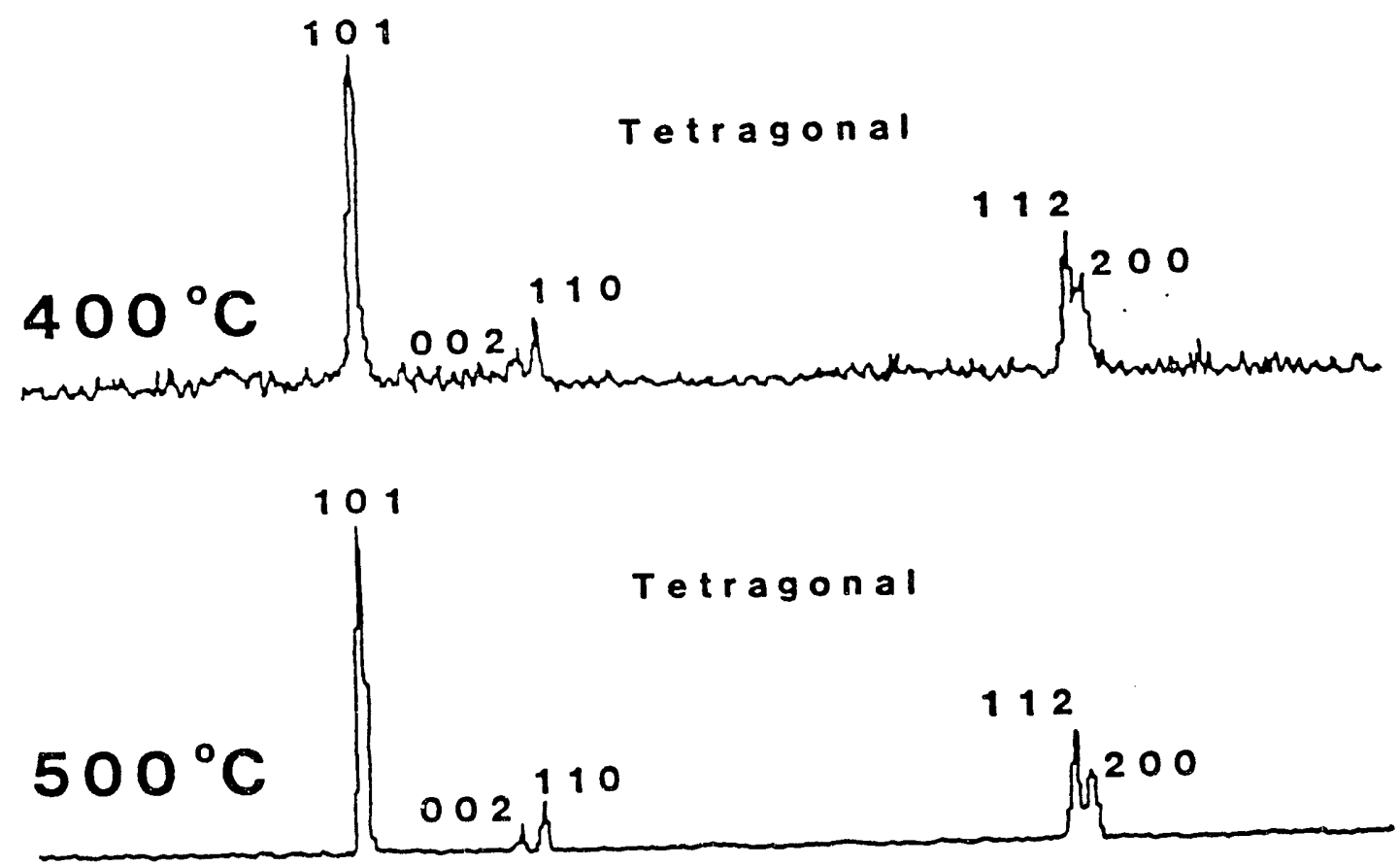

Figure 40. X-Ray diffraction patterns for zirconia membranes fabricated from sols prepared by the steric hindrance method. All membranes were heated at $2^{\circ} \mathrm{C} / \mathrm{min}$ to the indicated temperature and held at this temperature for $30 \mathrm{~min}$.

particles produced by both the solubility control and steric hindrance methods. For large particles, the monoclinic phase is the stable form at these relatively low temperatures. However, the tetragonal form appears to be more stable for nanoparticulate zirconia, sirce the monoclinic form has a larger surface excess energy than the tetragonal form [24]. 


\section{Mixed Oxide Membranes}

The steric hindrance method can be used to prepare sols containing mixed oxides of various compositions. Consequently, a study was conducted to determine the effect of composition on the thermal stabilities of mixed $\mathrm{TiO}_{2}-\mathrm{ZrO}_{2}$ membranes fabricated via the steric hindrance method. Earlier studies of mixed oxide membranes prepared by the other techniques indicated that the thermal stabilities of mixed oxide membranes were much improved over the thermal stabilities of the single phase membranes. However, these earlier studies involved only mixed oxide membranes with one or two compositions.

Figure 41 shows how the porosities of various mixed oxide membranes were affected by the firing temperature. Clearly, adding 5 to $208 \mathrm{ZrO}_{2}$ to a $\mathrm{TiO}_{2}$ membrane caused significant stabilization of the membrane porosity at firing temperatures up to $700^{\circ} \mathrm{C}$. Figure 42 also indicates the improvement in thermal stability obtained by adding $\mathrm{ZrO}_{2}$ to $\mathrm{TiO}_{2}$ membranes. While the mean pore size in pure $\mathrm{TiO}_{2}$ membranes increased past the microporous region (diameter $<2 \mathrm{~nm}$ ) when such membranes were fired to $350^{\circ} \mathrm{C}$, a $208 \mathrm{ZrO}_{2}-808 \mathrm{TiO}_{2}$ membrane still exhibited micropores even when fired to $500^{\circ} \mathrm{C}$ for $30 \mathrm{~min}$. (The $208 \mathrm{ZrO}_{2}$ membrane had a specific surface area of $228 \mathrm{~m}^{2} / \mathrm{g}$ and a porosity of 408 after firing at $500^{\circ} \mathrm{C}$.) However, further addition of $\mathrm{ZrO}_{2}$ was not beneficial. This increase in thermal stability has important implications for potential high temperature applications of these microporous ceramic membranes.

Figure 43 illustrates the effect of adding titania on the porosities of zirconia membranes, while Figure 44 shows the effect of adding titania on the mean pore diameters of the same membranes. A $108 \mathrm{TiO}_{2}-908 \mathrm{ZrO}_{2}$ membrane exhibited improved thermal stability over a pure $\mathrm{ZrO}_{2}$ membrane, but further 


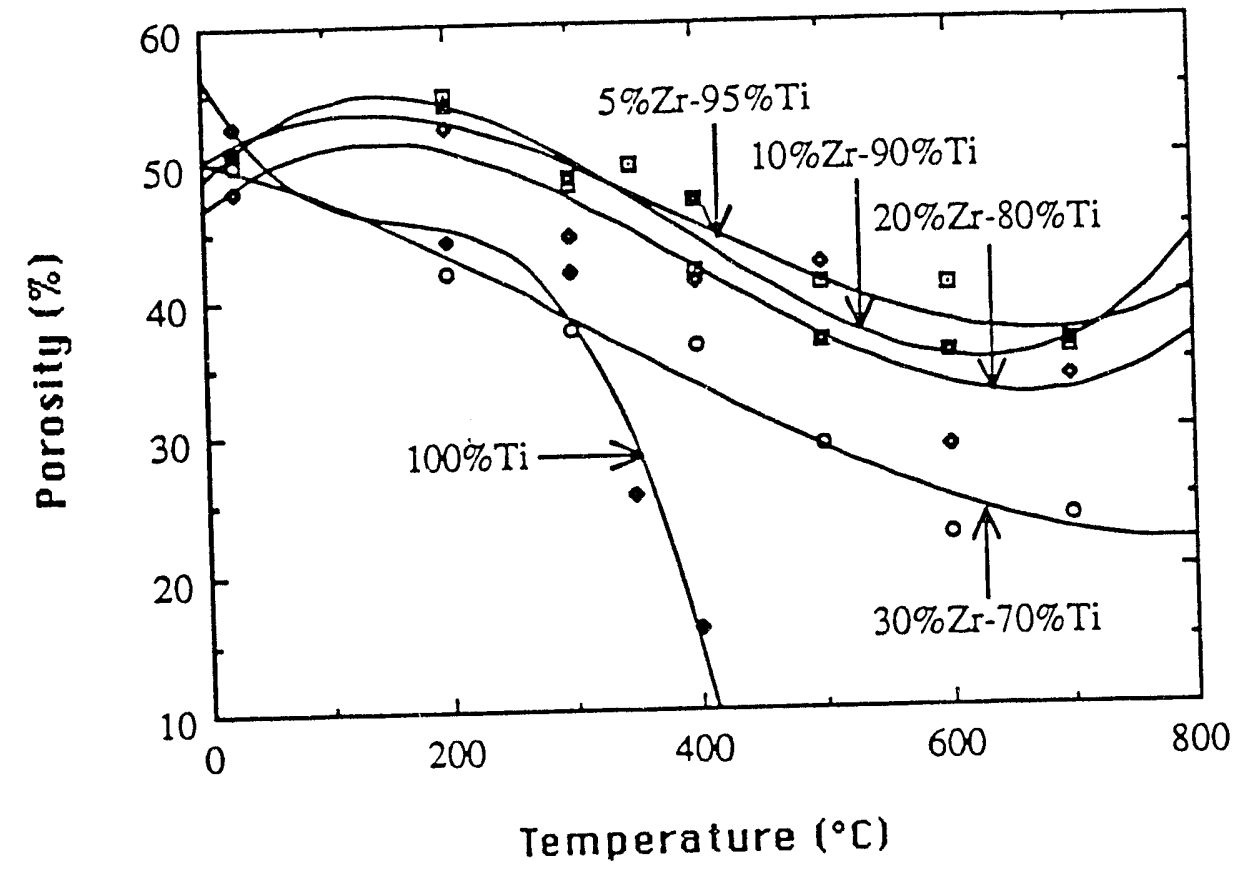

Figure 41. Porosities of pure $\mathrm{TiO}_{2}$ and mixed $\mathrm{ZrO}_{2}-\mathrm{TiO}_{2}$ membranes fabricated via the steric hindrance method as a function of firing temperature. All membranes were heated at $2^{\circ} \mathrm{C} / \mathrm{min}$ to the indicated temperature and held at this temperature for $30 \mathrm{~min}$.

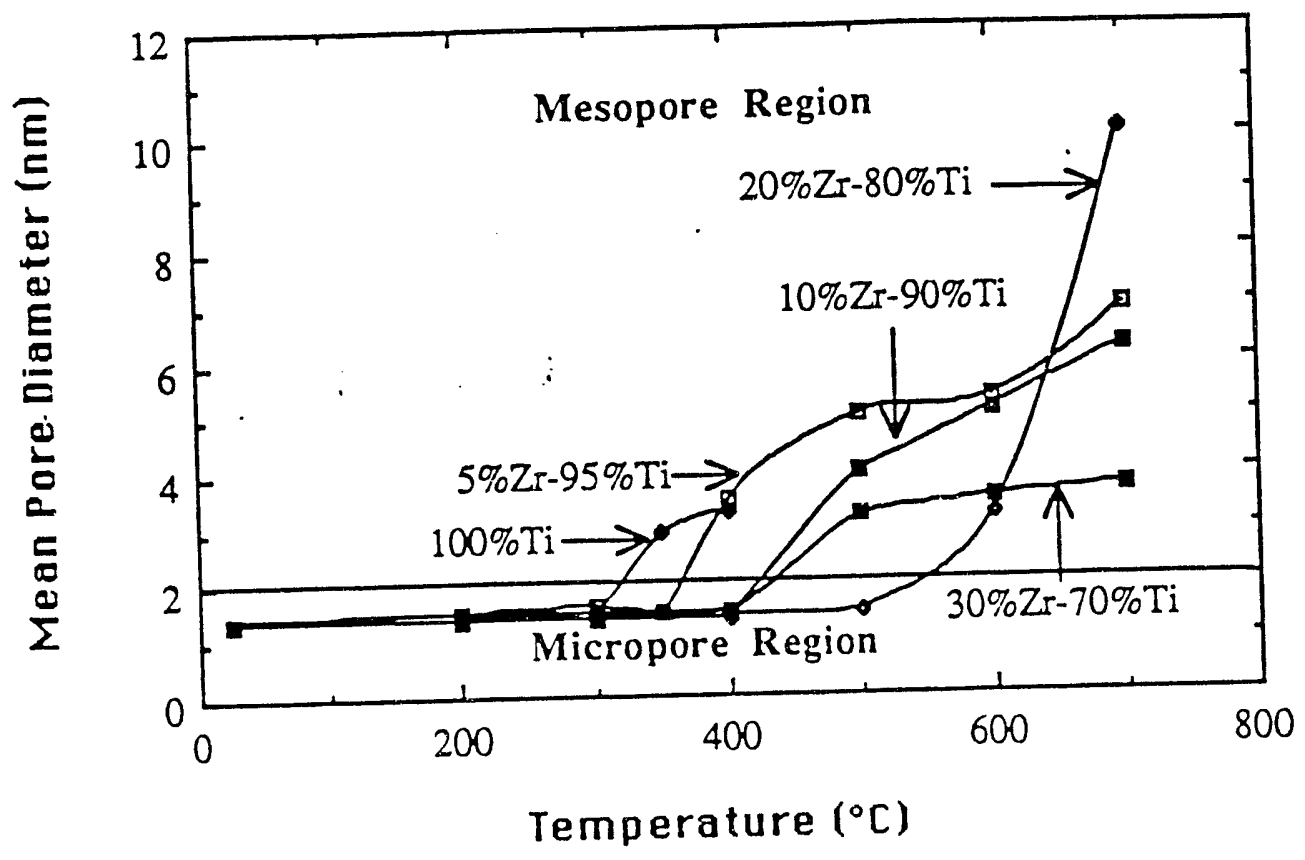

Figure 42. Mean pore diameters of pure $\mathrm{TiO}_{2}$ and mixed $\mathrm{ZrO}_{2}-\mathrm{TiO}_{2}$ membranes fabricated via the steric hindrance method as a function of firing temperature. All membranes were heated at $2^{\circ} \mathrm{C} / \mathrm{min}$ to the indicated temperature and held at this temperature for $30 \mathrm{~min}$. 


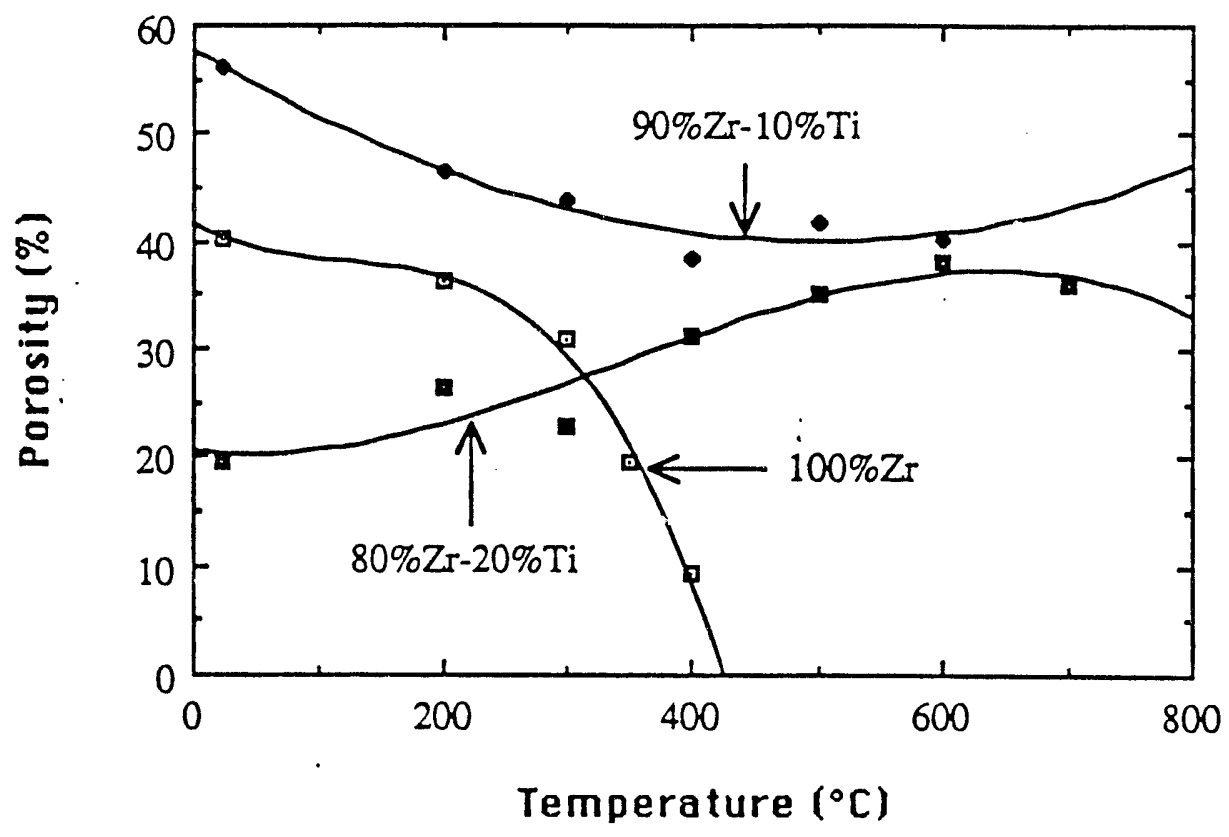

Figure 43. Porosities of pure $\mathrm{ZrO}_{2}$ and mixed $\mathrm{ZrO}_{2}-\mathrm{TiO}_{2}$ membranes fabricated via the steric hindrance method as a function of firing temperature. All membranes were heated at $2^{\circ} \mathrm{C} / \mathrm{min}$ to the indicated temperature and held at this temperature for $30 \mathrm{~min}$.

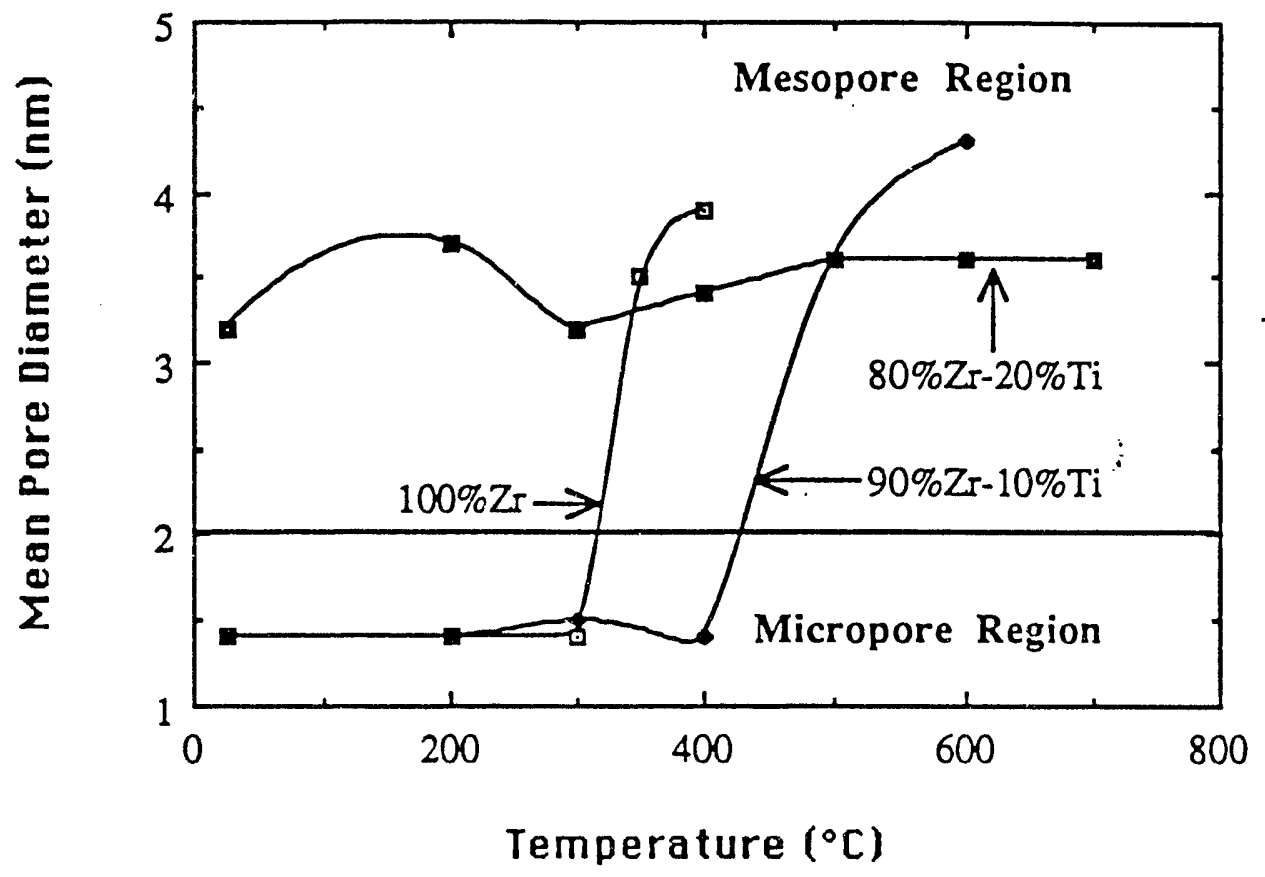

Figure 44. Mean pore diameters of pure $\mathrm{ZrO}_{2}$ and mixed $\mathrm{ZrO}_{2}-\mathrm{TiO}_{2}$ membranes fabricated via the steric hindrance method as a function of firing temperature. All membranes were heated at $2^{\circ} \mathrm{C} / \mathrm{min}$ to the indicated temperature and held at this temperature for $30 \mathrm{~min}$. 
addition of $\mathrm{TiO}_{2}$ was not beneficial. In fact, a $20 \% \mathrm{TiO}_{2}-80 \% \mathrm{ZrO}_{2}$ membrane did not exhibit any evidence of microporosity. While we suspect that the observed changes in properties may be related to changes in the crystal structures of these membranes, we have no direct evidence to support this assumption. X-ray diffraction studies of these membranes as a function of firing temperature and dwell time would need to be performed in order to obtain more information about the crystalline phases that are present in these systems. Although a detailed X-ray diffraction study was not performed, differential thermal analysis (DTA) diagrams obtained for these membranes (see Figure 45) did correlate in certain cases with the observed thermal stabilities of the membranes. The sharp exothermic peaks shown in Figure 45 indicate that crystallization occurred. The improvement in thermal stability noted in changing from a pure $\mathrm{ZrO}_{2}$ membrane to a $108 \mathrm{TiO}_{2}$ - $908 \mathrm{ZrO}_{2}$ membrane is consistent with the higher crystallization temperature exhibited by the $108 \mathrm{TiO}_{2}$ membrane (see Figure 45). The improved thermal stabilities obtained by adding $\mathrm{ZrO}_{2}$ to $\mathrm{TiO}_{2}$ membranes also correlated well with the higher crystallization temperatures revealed by the DTA analysis (see Figure 45). In fact, for the membrane with the best thermal stability (208 $\left.\mathrm{ZrO}_{2}-808 \mathrm{TiO}_{2}\right)$, the DTA scan did not provide any evidence for crystallization until a temperature of $700^{\circ} \mathrm{C}$ was reached. However, addition of more than $208 \mathrm{ZrO}_{2}$ did not further enhance the thermal stability of the membranes, even though the crystallization temperatures for these membranes were still about $700^{\circ} \mathrm{C}$. In these cases, though, the shoulder in the diagrams near $225^{\circ} \mathrm{C}$ may indicate that other changes in crystal phases occurred at much lower temperatures. Such changes may have detracted from the thermal stabilities of these membranes. 


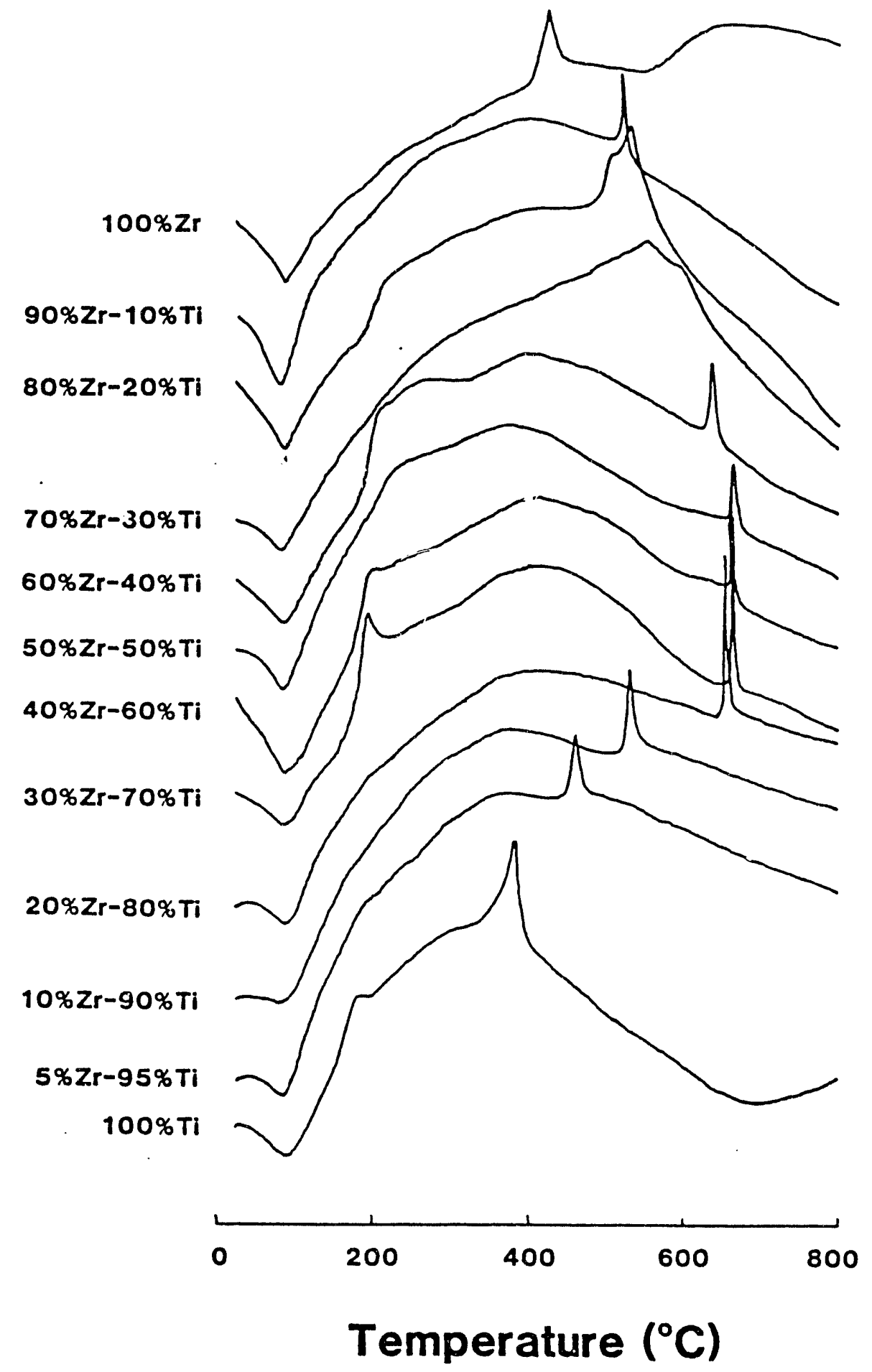

Figure 45. Differential thermal analysis diagrams of mixed $\mathrm{TiO}_{2}-\mathrm{ZrO}_{2}$ membranes heated at $2^{\circ} \mathrm{C} / \mathrm{min}$. The composition of each sample has been indicated. $\alpha-\mathrm{Al}_{2} \mathrm{O}_{3}$ was used as a reference. 


\section{v. Fabrication of Supported Membranes}

\section{A. Coating Techniques}

To characterize the permeabilities and permselectivities of ceramic membranes, the precursor sols must be placed on a porous support. This approach is needed because most unsupported ceramic membranes are too weak to withstand the pressures generated when such membranes are placed in a test apparatus. Since the porous supports used for these studies were shaped like test tubes and the membranes were to be placed on the outside of these supports, two techriques could be used to place the membranes on the supports: "slip casting" and dip coating.

Technically, slip casting involves pouring a suspension into an absorbent mold which draws off most of the liquid, leaving the casting to dry in place. This technique was employed to prepare our porous supports. It could also be used to place membranes on the interior surfaces of these supports. A variant of this technique was used to place membranes on the outside surfaces of these supports. A dry porous support was dipped into a precursor sol so that the solvent would be drawn into the support. It was anticipated that this approach would cause particles of the sol to deposit on the outside of the support. However, the initial studies involving this approach indicated that the sol particles were so small that they were being drawn into the body of the support, where they were being deposited at random [1]. Coherent membranes were not formed.

Subsequent studies of supported membranes employed dip coating to place the membrane on the support. In dip coating, a non-porous support material is 
placed in the sol and particles adhere to the support. In order to mimic the behavior of a non-porous support and to minimize the potential for imbibition of the sol by the support, the porous clay supports were wetted with either water or alcohol (depending on the composition of the sol) prior to dipping. This technique for fabricating supported membranes was later shown to be a coating process rather than a slip casting process (refer to the discussion in Section VI.A.2).

Our early studies of supported membranes employed a hand dipping procedure to coat the membranes. In this process, the wetted supports were placed in the sol for ca. $20 \mathrm{sec}$, withdrawn manually, and air dried before further treatment. However, we were concerned about an apparent lack of reproducibility among measurements obtained with these coated supports. Some of the variability was attributed to differences in the rates of withdrawal of the supports from the sols that would be expected when this hand dipping procedure was used. Therefore, an apparatus was constructed in which the supports were attached to a motor turning at constant speed. The supports could then be withdrawn from the sol in a reproducible manner (a withdrawal speed of $16.7 \mathrm{~cm} / \mathrm{min}$ was used most often). Using this approach, we have prepared supported, permselective ceramic membranes of alumina and titania with reproducible properties $[2,3]$.

We also employed two other techniques for coating supports, although the supports being coated were typically non-porous and were used for applications other than separations. One technique was spin coating as used in the semiconductor industry to coat silicon chips. With this technique, we placed enough sol (typical sol concentrations were ca. $28 \mathrm{w} / \mathrm{w}$ or higher) on a flat support (e.g., a microscope slide) to cover the surface and spun the entire 
system until it apperred to be dry. While the conditions used for spinning depend on factors such as the viscosity of the sol and the nature of the support, good coatings were often achieved by spinning at ca. $1500 \mathrm{rpm}$ for one minute or less.

We also used a second technique that combined spin coating and dip coating. With this approach, the sol used for coating (typically $30 \mathrm{~mL}$ at a concentration of ca. $28 \mathrm{w} / \mathrm{w}$ or higher) was placed in a rectangular trough with a semi-circular lip cut out of it at one end. The piece to be coated was a glass tube that was closed at the end in the trough and had an indentation at the other end which fit in the cut-out lip of the trough. This tube was attached to a motor and spun around a horizontal axis (0.5-8 rpm, although these rotation speeds do not appear to affect the thickness of the coating) so that part of the outer surface of the tube was always in the sol during the coating process. Such slow spinning rates were needed to allow droplets of the sol to fall off the tube. When faster rotation speeds were utilized, droplets of sol tended to adhere in spots to the support, thereby producing an uneven coating. When the rotation speed was slow, uniform coats could be deposited in about $5 \mathrm{~min}$.

The advantage of this last technique is that it can be scaled up readily, whereas spin coating can only be applied over a limited area (ca. 75 $\mathrm{cm}^{2}$ with our spinning apparatus). While this technique might also be adapted to coat our porous, test tube shaped supports, such adaptations have not been attempted to date because dip coating these supports is more convenient for laboratory scale studies. 
B. Drying Problems

Most of our supported membranes have been fabricated from conventional sols using the dip coating process described above. Electron photomicrographs of these supported membranes indicate that, during drying, the supported $\mathrm{TiO}_{2}$ membranes developed many more cracks than the supported alumina membranes [2]. This problem can be minimized by controlling the humidity of the drying atmosphere and by extending the drying process over a long period of time. Even when these precautions were taken, cracks still formed in the $\mathrm{TiO}_{2}$ membranes. This observation explains the poorer permselectivities of supported titania membranes as compared to supported alumina membranes.

When nanoparticulate sols are used to form supported membranes, the small pore sizes of these membranes should induce even larger capillary forces within the membranes during drying than develop during the drying of membranes formed from conventional sols. Thus, one would expect crack formation to be very severe in nanoparticulate membranes. This expectation, was realized in practice. We have been unable to form crack-free surposted membranes from nanoparticulate titania sols even when the resulting modules were dried under controlled humidity conditions over periods as long as two months.

As a result, other preparation techniques are now being considered for the fabrication of supported nanoparticle membranes. Since we are still in the initial stages of exploring some promising techniques, we are not yet able to report on any of these studies. The results described in the following section have been obtained by studying ceramic membranes formed from conventional sols of titania and alumina which have then been deposited on porous clay supports. 
VI. Properties of Supported Membranes

Supported membranes have been characterized by measuring the flux of pure water through the membranes (normalized by the apparent exterior surface area of the support) and the permselectivity of the membranes to various test species. All permeation rate and permselectivity measurements were made using a laboratory scale apparatus for determining flow rates [5,25] in which a pressure cell held the supported membrane. Cajon fittings allowed a rubber ' $O$ '-ring to be compressed against both the supported membrane and the housing. These fttings provide pressure-tight isolation of the feed (high pressure) and permeate (low pressure) streams [1]. The entire test loop was operated with total recycle of both retentate and permeate streams so as to maintain a constant composition of the feed stream.

\section{A. Permeate Flux Measurements}

Permeation rates measured with this apparatus were used to determine the effects of several factors (e.g., dipping and firing treatments employed in preparing the supported membrane, $\mathrm{pH}$ and temperature of the feed stream) which influence the behavior of these supported membranes. Most of the supports used in these studies were prepared from the original clay formulation described on p. 7 of this report. These formulations did not contain the additives that were added later in attempts to prepare more reproducible clay supports. All total permeate flux values were normalized by dividing the tctal throughput by the external surface area of the support employed. The effects of each factor on the permeation rate will be described separately. 
1. Coating Material and Number of Dippings

Permeation rate measurements were conducted for a series of supported alumina and titania membranes of various thicknesses. The sols used to prepare the membranes had the following compositions:

Boehmite Sol

$0.5 \mathrm{M}$ total Al

$0.035 \mathrm{M} \mathrm{HNO}_{3}$

$10^{-5} \mathrm{M} \mathrm{H}_{3} \mathrm{PO}_{4}$

$\mathrm{pH} 3.9-4.0$

\section{Titania Sol}

$0.278 \mathrm{M}$ total $\mathrm{Ti}$

$0.111 \mathrm{M} \mathrm{HNO}_{3}$

$\mathrm{pH} 1.3$

The supports were first placed in a beaker of water to saturate the pores. Then the water soaked supports were dipped into the sols and withdrawn by hand. (Later studies indicated that use of a mechanized withdrawal apparatus allowed us to prepare supported membranes with better permselectivities.) Coated supports were air dried at $60^{\circ} \mathrm{C}$. This dipping and drying cycle was repeated three more times. After four repetitions of this cycle, the supported membrane was heated at $1.7^{\circ} \mathrm{C} / \mathrm{min}$ to either $400^{\circ} \mathrm{C}$ (titania) or $500^{\circ} \mathrm{C}$ (alumina) and held at the firing temperature for one hour to fix the membrane. SEM photomicrographs of the resulting supported membranes suggested that these four cycles ultimately led to membranes with total thicknesses of ca. 1.0 and $0.6 \mu \mathrm{m}$ for alumina and titania, respectively [2].

Evidence that these systems behaved linearly with respect to the number of dipping cycles was provided by checking the permeation rates of pure water through the supported membranes at an operating pressure of $100 \mathrm{psig}$. For a conventional membrane transport model, in which the resistance of the support is presumed to be negligible, the permeation rate of a solvent should be inversely related to the membrane thickness. If each dipping adds a uniform increment to the membrane thickness, the permeate flux should be inversely 
related to the number of dips. However, in practical systems the resistance to transport through the supported membrane consists of two contributions, one due to the resistance of the support and the other to the resistance of the membrane proper. Linear behavior can only be obtained if the resistance of the support is negligible compared to the membrane or if one corrects for the resistance of the support.

When such a correction was applied to the permeate flux data [26], the plots shown in Figure 46 were obtained. For $\mathrm{Al}_{2} \mathrm{O}_{3}$ membranes the resistance of the support was roughly equivalent to that resulting from two dipping and drying cycles, while for $\mathrm{TiO}_{2}$ the resistance of the support was considerably greater, namely 17 to 21 cycles $[2,5]$. Similar results (support resistance equivalent to 4 to 5 cycles for supported $\mathrm{Al}_{2} \mathrm{O}_{3}$ membranes and 8 to 13 cycles for $\mathrm{TiO}_{2}$ ) were obtained when this experiment was repeated a few months later. Supported $\mathrm{TiO}_{2}$ membranes are clearly more permeable than $\mathrm{Al}_{2} \mathrm{O}_{3}$ membranes.

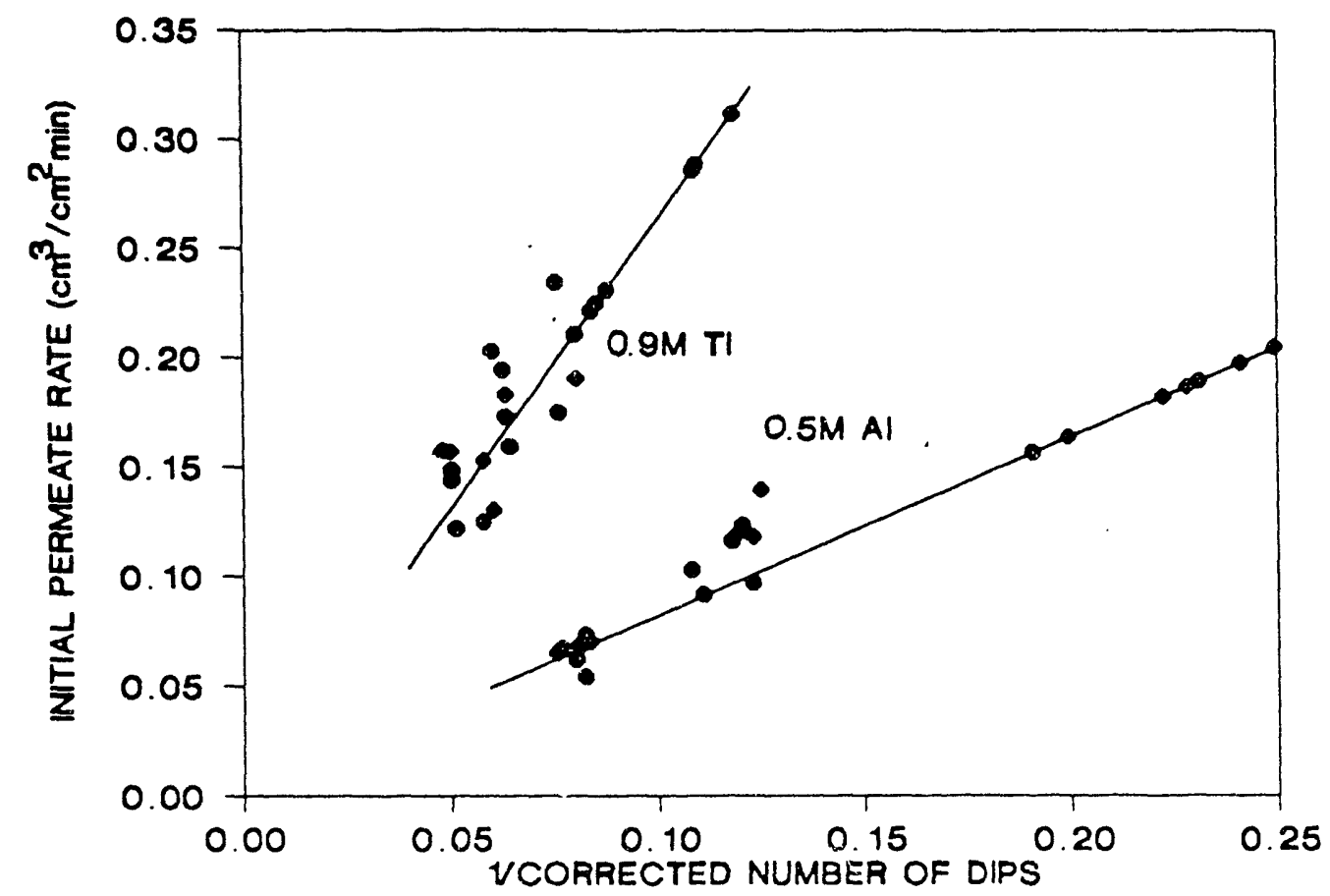

Figure 46. Permeate flux data at $100 \mathrm{psig}$ and $25^{\circ} \mathrm{C}$ for supported alumina and titania membranes. 
This result is attributed to the fact that cracking of the membranes is a much more severe problem for $\mathrm{TiO}_{2}$ membranes than $\mathrm{Al}_{2} \mathrm{O}_{3}$ membranes [2].

\section{Dipping and Firing Treatments}

Water permeabilities were also used to investigate the effects of other fabrication parameters on the properties of the resulting supported membranes. The major question studied was whether the membrane had to be fired after each coating in order to affix the membrane to the support or whether multiple coatings were possible between firings. Since the previous study of permeation rate through the supported membrane as a function of the number of dippings involved as many as twenty separate dippings, it was clearly important to answer this question.

Our initial study compared permeation rates of water through supported alumina membranes prepared by two different techniques. One membrane was fired after each dipping until it had been coated four times. The other membrane was dipped and dried four separate $t$ imes before firing. Although the flux of water through the first membrane was somewhat lower than the flux through the second membrane, the flux through the first membrane was equivalent to that expected for 4.75 separate dipping and drying cycles before firing. This small difference suggests that most of the sol stayed on the membrane after each dipping and drying step. Thus, firing the membrane after every four dips is a reasonable approach to build up the thickness of membrane necessary to achieve useful permselectivity properties [2].

Because of the importance of this result and questions about the reproducibility of the supports used in this initial study, the study was repeated 
using six clay supports that had similar permeation rates for pure water before being coated. Three of these supports were subjected to five separate coating and drying cycles using an alumina sol (the same composition as in the initial study) before being fired. The other three supports were fired after each coating with the alumina sol until five coats were applied. Again, the water permeation rates through all of the supported membranes were quite similar, indicating that there is no substantial practical difference between these two methods of coating [3].

In preparing the supported membranes, the clay supports were saturated with water before they were dipped into the sol. This approach was taken in order to mimic a coating process rather than a slip casting process. Several experiments were undertaken which verified that these systems were being coated, not slip cast.

When supports are coated, the thickness of the coating is independent of the contact time between the sol and the support [27]. For the dipping technique used to prepare these supported membranes, dipping for periods of 20 , 200 and $2000 \mathrm{sec}$ gave essentially the same percentage loss of flow after firing. This loss was determined from measurements of the permeation rates of water through the coated membranes. Only when a support was dipped for 20,000 sec was enough extra material deposited on the supported membrane to cause a significantly greater percentage loss of flow [2].

If the supports are being coated, the thickness of the coating should depend on the viscosity of the sol that is being applied [27]. Since the sol viscosity is proportional to the concentration of particles in the sol, one would expect to obtain thicker coatings (and lower water permeabilities) when using more concentrated sols. Figure 47 depicts the results of several exper- 


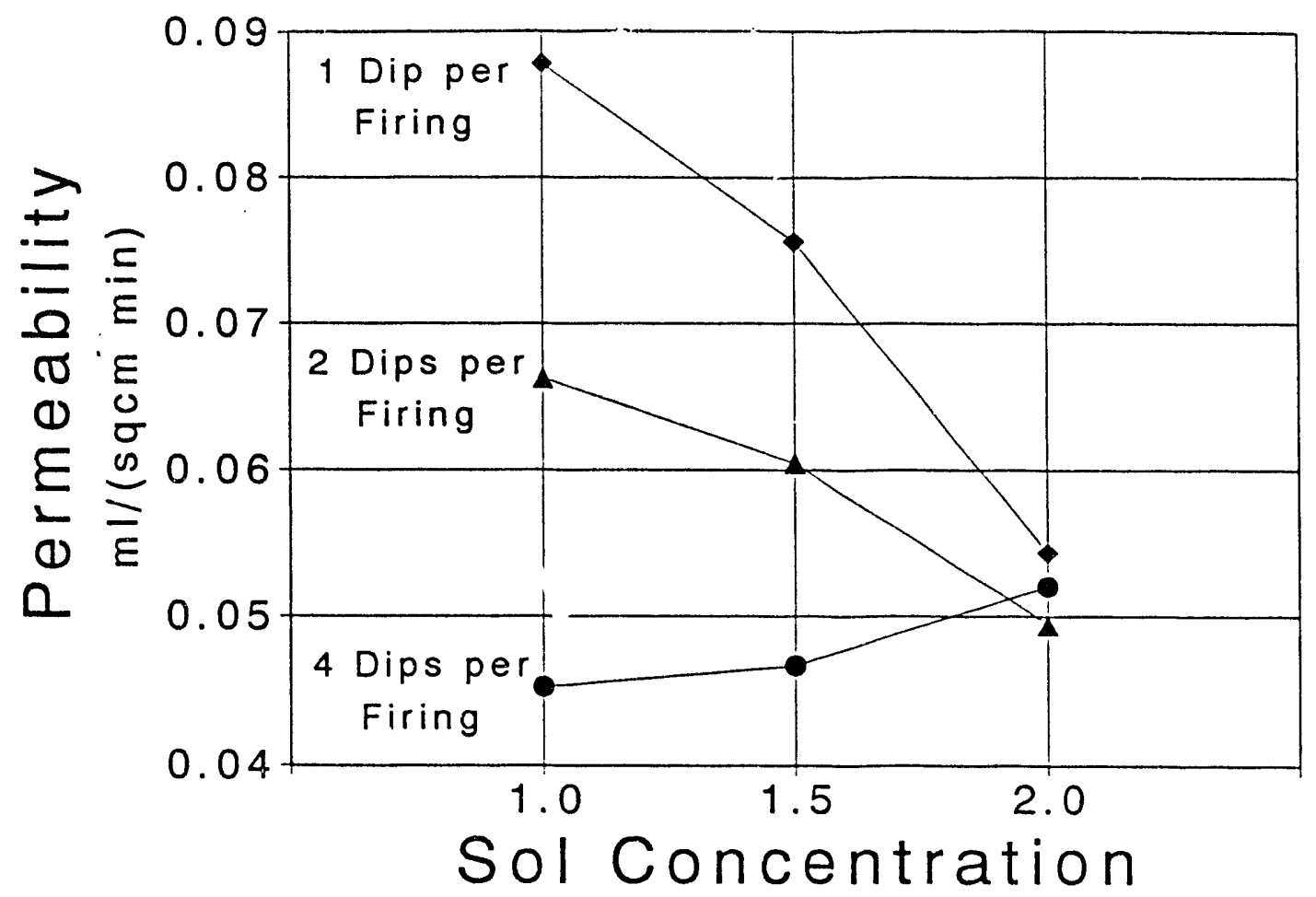

Figure 47. Permeability data at 100 psig and $25^{\circ} \mathrm{C}$ versus sol concentration with the number of dippings per firing as a parameter.

iments that were undertaken to verify this hypothesis. Note that the alumina sol used to coat the clay supports in these experiments has the same composition ( $0.5 \mathrm{M}$ total $\mathrm{Al}$ ) as the sol described earlier, except that this sol contains no phosphate. Thus, the $x$-axis in this figure is really a sol concentration factor, and the $\mathrm{x}$-axis value of 1.0 corresponds to $0.5 \mathrm{M}$ total $\mathrm{Al}$. When the supports were dipped either once or twice in the sols between firings, the fluxes of pure water through the supported membranes did indeed decrease dramatically as the concentration of the sol was increased. This result indicates that, when higher sol concentrations were employed, more material was deposited on the support during each dipping step. However, the expected trend was not observed when the supports were dipped four times between firings. We attributed this unexpected result to the membrane 
becoming so thick that it cracked due to stresses induced during the drying and firing steps of the preparation protocol. Support for this suggestion is provided by noting that membranes formed from sols which had been concentrated by a factor of 3 were so thick after only a single dipping that they cracked and flaked off while drying [25].

If the sol is being coated on the wetted support, the coating thickness should increase as the speed of withdrawal of the support from the sol is increased. (Although this statement is somewhat counterintuitive, note that slow withdrawal rates allow more time for the sol to drain off the support, thus producing thinner coatings.) Since an apparatus had been fabricated for the mechanical withdrawal of supports from sols, this prediction could also be tested. Permeability and rejection data for tests of both water and an aqueous solution containing 1 wto polyethylene glycol (molecular weight of 1000) are listed in Table XVII.

As shown by these data, varying the speed of withdrawal of the support from the precursor sol by a factor of two around the selected value of 16.7 $\mathrm{cm} / \mathrm{min}$ had little effect on the properties of the resulting coating. Although the withdrawal rates during manual withdrawal were highly variable, these

Table XVII. Effects of withdrawal velocity of the support on properties of supported alumina membranes at $25^{\circ} \mathrm{C}$.

\begin{tabular}{|c|c|c|c|c|}
\hline $\begin{array}{l}\text { Withdrawal Speed } \\
(\mathrm{cm} / \mathrm{min})\end{array}$ & $\begin{array}{c}\text { Permeate } \\
\text { Water }\end{array}$ & $\begin{array}{c}\text { Flux }\left(\mathrm{cm}^{3} / \mathrm{cm}^{2} / \mathrm{min}\right) \\
\text { DEG Solution } \\
\end{array}$ & PEG & $\begin{array}{l}\text { Rejection } \\
(8) \\
\end{array}$ \\
\hline 7.9 & 0.061 & 0.023 & & 50.6 \\
\hline 16.7 & 0.058 & 0.022 & & 57.5 \\
\hline 34.1 & 0.056 & 0.017 & & 58.9 \\
\hline manual & 0.057 & 0.021 & & 34.7 \\
\hline
\end{tabular}

All supports were subjected to three cycles, each of which involved four dippings and one firing. 
velocities were always much higher (ca. several hundred $\mathrm{cm} / \mathrm{min}$ ) than the velocities obtained with the mechanized withdrawal apparatus. Thus, one would expect supports withdrawn manually to have somewhat thicker coatings and lower permeabilities (and probably better permselectivities) than supports withdrawn mechanically. However, for reasons that are not known, this expectation was not realized in practice. It is nonetheless clear that mechanized withdrawal of the supports is helpful in efforts to optimize the performance of the supported membranes [3].

3. $\mathrm{pH}$ of Feed Stream

The performance of supported membranes was expected to depend on the $\mathrm{pH}$ of the feed stream. A study was performed to determine the stability of supported membranes that were exposed to test solutions at extreme pH values. It was also possible that the nature of the membrane itself was affecting the permeabilities of test solutions at different $\mathrm{pH}$ values. Unlike most polymeric membranes, ceramic oxide membranes should develop a charge when in contact with aqueous test solutions. The magnitude of this charge depends on the $\mathrm{pH}$ and ionic strength of the surrounding medium $[18,19]$. If the pores of the membrane are small enough, this charged membrane surface should have an observable effect on the flow rate of an electrolyte solution. These two questions were investigated in separate experiments. 


\section{a. Stability of Supported Membranes}

\section{Procedures}

In order to determine the $\mathrm{pH}$ stability of supported and unsupported alumina and titania membranes, as well as the stability of the underlying clay supports, these materials were exposed to aqueous test solutions at different $\mathrm{pH}$ values for 10 weeks. Degradation of supported membranes was inferred by comparing the membrane permeabilities to pure water before and after the test period. Dissolution of unsupported membranes and clay supports was measured by determining metal concentrations in solution following exposure to the test solution for the time frame of interest.

The protocol utilized for measuring the flux of deionized water through the supported membranes is listed below.

1. A number of supported alumina and titania membranes with macroscopic external surface areas ranging from 11 to $24 \mathrm{~cm}^{2}$ were prepared. Permeation rates of water through these membranes were measured after dipping the supports into the precursor sol 0,4 and 8 times and then firing at an appropriate temperature.

2. Supported membranes were placed in $500 \mathrm{~mL}$ of aqueous $0.1 \mathrm{M}$ $\mathrm{NaClO}_{4}$ solutions and held at room temperature $\left(22^{\circ} \mathrm{C}\right)$ in the dark in polypropylene containers. Initial solution $\mathrm{pH}$ values of $2.0,3.5,6.3$, $8.4,9.9$, and 12.0 were obtained by adding $\mathrm{HClO}_{4}$ or $\mathrm{NaOH}$. Buffered $\mathrm{pH}$ solutions were not used because these solutions often contain chemicals which strongly adsorb on oxide materials (e.g., phosphate buffers for $\mathrm{pH}$ 
7) and change the charge on the particles. These solutions were considered to be saturated in atmospheric gases.

3. The $\mathrm{pH}$ values of the solutions were measured periodically and adjusted as necessary to maintain a desired $\mathrm{pH}$ range. This adjustment was required only for the solutions at $\mathrm{pH} 8.4$ and 9.9.

4. Twice weekly the solutions were lightly agitated to maintain a homogeneous aqueous phase.

5. After 5 weeks, permeation rates of water through the supported membranes were measured, and the test solutions were then replaced by fresh solutions.

6. Following an additional 5 week exposure to the test solutions (10 weeks total), the membranes were removed from their solutions and dried. The fluxes of water through these membranes were then measured. It was necessary to dry the membranes because earlier experiments had shown that the permeation rates change significantly when initially dry membranes are left in contact with test solutions. To ensure comparable permeability measurements, the following technique was used to dry the wet membranes:

a. flush for 30 minutes with water at 150 psig to remove residual solution;

b. soak in pure water for 5 days to minimize any residual charge that may have formed on the membrane;

c. dry to $60^{\circ} \mathrm{C}$ overnight, then dry to $250^{\circ} \mathrm{C}$ for one hour to remove any free or adsorbed water. (Separate experiments indicated that this treatment restored supported membranes to near their original dry state.) 
7. Permeation rates measured in steps 5 and 6 were compared to the pre-test fluxes recorded after 0,4 and 8 dippings (the open circles in Figure 48). The equivalent amount of membrane remaining after steps 5 and 6 was estimated by interpolation using the permeation rate data (see Figure 48). Membrane losses were calculated as:

$$
\text { Membrane loss }(8)=100 *\left(8-N^{\prime}\right) / 8
$$

The protocol followed to obtain measurements of dissolved metal concentrations for unsupported membranes and clay supports is listed below.

1. Unsupported membranes and clay supports were crushed to ca. 20-100 mesh size to achieve efficient contact with the test solution.

2. Crushed materials were placed in separate containers with the solutions described in the second step of the previous protocol. The concentration of solids in the test solutions was ca. $200 \mathrm{ppm}$ (w/w).

3. The same procedure was followed as described in steps 3 and 4 of the previous protocol, except that the solution $\mathrm{pH}$ was not adjusted to hold a constant $\mathrm{pH}$.

4. After 10 weeks of exposure, the supernatant liquids were decanted from any remaining solids. The decanted solutions were then analyzed for dissolved metals.

5. Losses from the unsupported membrane and the clay support were calculated as :

$$
\text { Membrane Loss }(8)=100 * C_{f} / C_{0}
$$




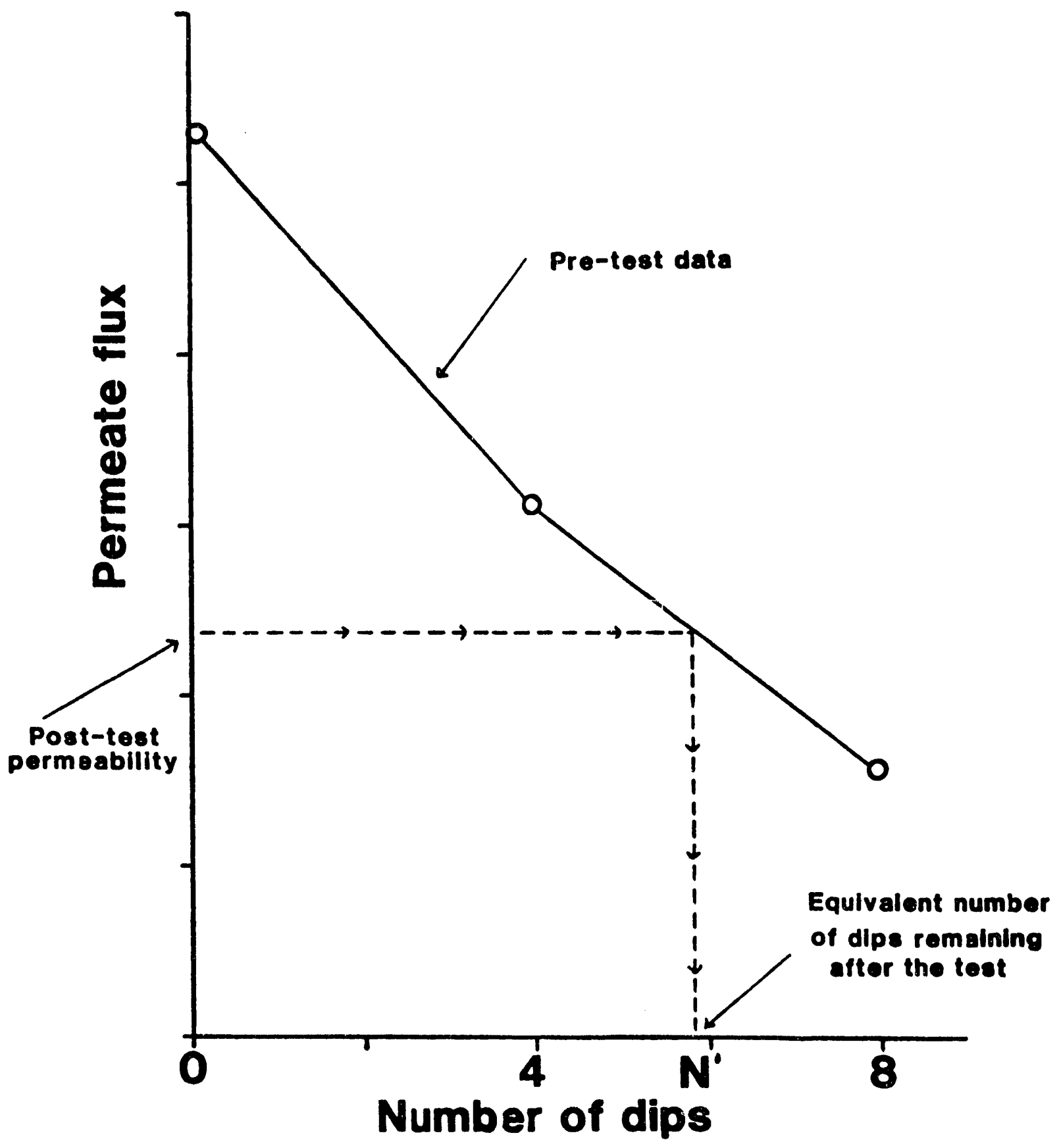

Figure 48. Procedure for determining membrane losses from supported membranes using permeability measurements. 
where $C_{f}$ is the final concentration of dissolved metal. $C_{0}$ is an estimate of what the dissolved metal concentration would be if all the solid were to dissolve. For the alumina and titania membranes, $C_{0}$ was determined by multiplying the actual concentration of solids in the suspension by the weight percent of the metal of interest in the membrane ( $538 \mathrm{Al}$ for alumina and $418 \mathrm{TI}$ for titania). Since the elemental compositions of the two clays used in preparing the clay supports are known, $C_{0}$ can also be determined for the elements in these supports.

\section{Results}

Although the test solutions for dissolution of the unsupported membranes and clay supports were not held at a constant $\mathrm{pH}$, the $\mathrm{pH}$ of these systems was measured often during the study. In all cases, systems which were initially at $\mathrm{pH} 2.0$ and 12.0 exhibited little change in $\mathrm{pH}$ over a 10 week period.

Unsupported titania membranes and clay supports behaved similarly at the other $\mathrm{pH}$ values. Test systems at $\mathrm{pH} 3.5$ and 6.3 had little change in $\mathrm{pH}$. Systems at $\mathrm{pH} 8.4$ and 9.9 both fell to about $\mathrm{pH} 7$ within 3 weeks of contact and then remained relatively constant. This behavior was attributed to absorption of atmospheric $\mathrm{CO}_{2}$, a weak acid, by the basic solutions. Not enough $\mathrm{CO}_{2}$ was absorbed by the solutions set to $\mathrm{pH} 2$ or 12 to affect their $\mathrm{pH}$ values.

Unsupported alumina membranes exhibited more complex behavior, as shown in Figure 49. After 3 weeks, the system which was initially at $\mathrm{pH} 3.5$ had risen to $\mathrm{pH} 4.7$ and remained at this $\mathrm{pH}$ for the remainder of the experiment. The remaining systems all equilibrated near $\mathrm{pH}$ 7. Although the drop in $\mathrm{pH}$ 


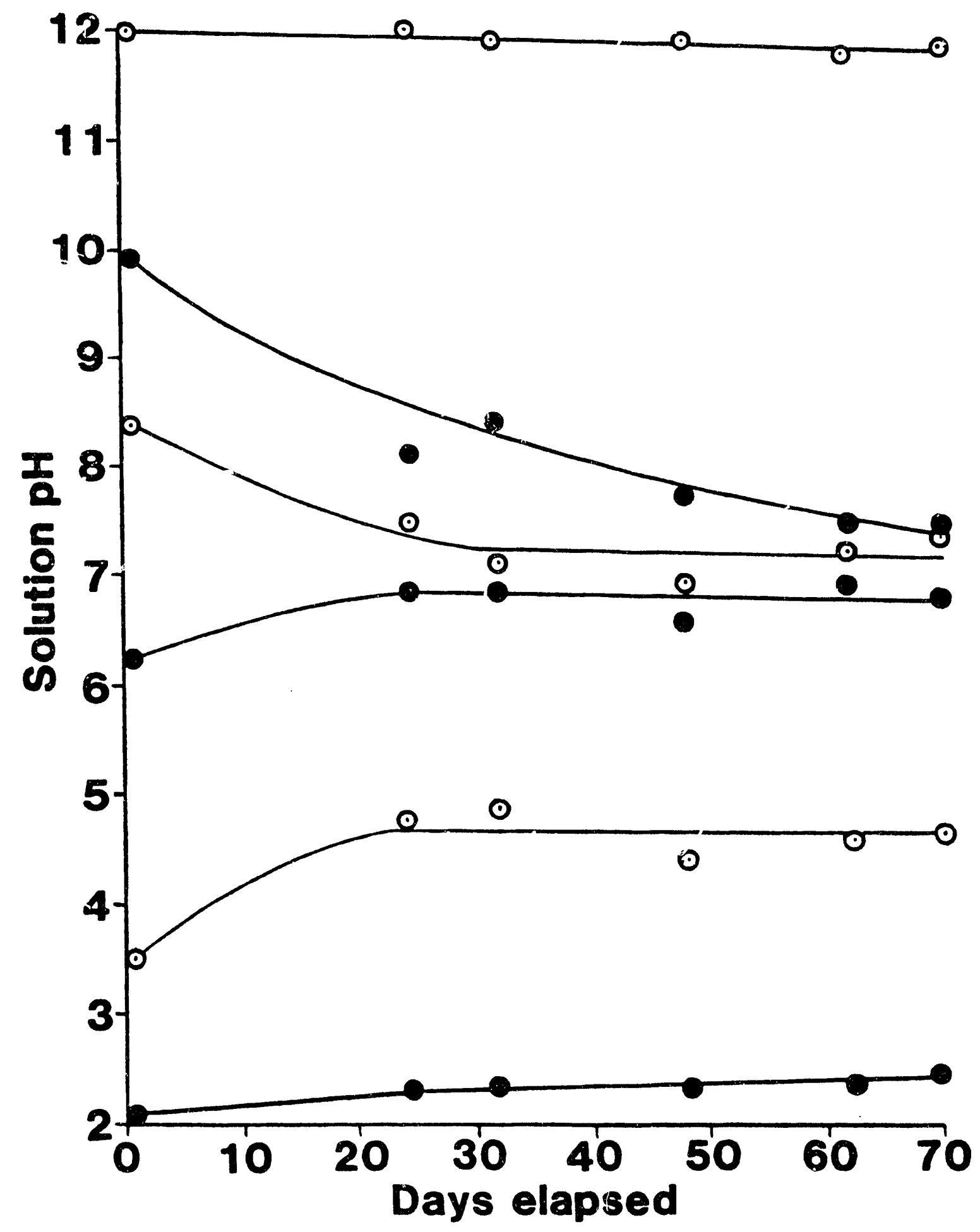

Figure 49. Variation of solution $\mathrm{p}^{\mathrm{H}}$ with time for unsupported alumina membranes exposed to test solutions at various $\mathrm{pH}$ values. 
values from 8.4 and 9.9 can again be attributed to $\mathrm{CO}_{2}$ absorption, the rise in $\mathrm{pH}$ from 3.5 and 6.3 probably indicates an interaction between the membrane surface and the surrounding solution. The resulting chemical reactions cause the solution $\mathrm{pH}$ to approach the isoelectric point of the membrane particles, which should lie between $\mathrm{pH} 7.0$ and 8.5 for alumina. This effect also contributes to the decline in $\mathrm{pH}$ observed in the basic test solutions.

Test solutions for the supported membranes showed similar $\mathrm{pH}$ changes. However, in these systems the solution $\mathrm{pH}$ was adjusted every few days to hold the $\mathrm{pH}$ nearly constant.

Figures 50 and 51 , for alumina and titania membranes respectively, show the effect of the solution $\mathrm{pH}$ on the amount of membrane loss ove: a 10 week period. Because of the $\mathrm{pH}$ changes discussed above, a time-weighted average solution $\mathrm{pH}$ value was used. For unsupported alumina membranes, significant dissolution was observed only at the extreme $\mathrm{pH}$ values of 2 and 12 . This dissolution under strongly acidic and basic conditions is not very surprising when one considers that these membranes are prepared by firing alumina gels at $500^{\circ} \mathrm{C}$. Several references indicate that $\boldsymbol{\gamma}$-alumina is formed at $500^{\circ} \mathrm{C}$ rather than the less soluble $\alpha$-alumina. Higher firing temperatures would be needed to form $\alpha$-alumina, but such temperatures would also lead to larger pore sizes and less exposed surface area for the membranes. Since larger pore sizes are undesirable for these membranes, a trade-off between $\mathrm{pH}$ stability and pore size may be necessary in applications involving alumina membranes.

At all $\mathrm{pH}$ values (including those at which the unsupported membranes were stable), the supported alumina membranes showed signicicant membrane loss, as estimated by the permeate flux measurements. Losses greater than 1008 indicate that the flux through the supported membrane after exposure to 


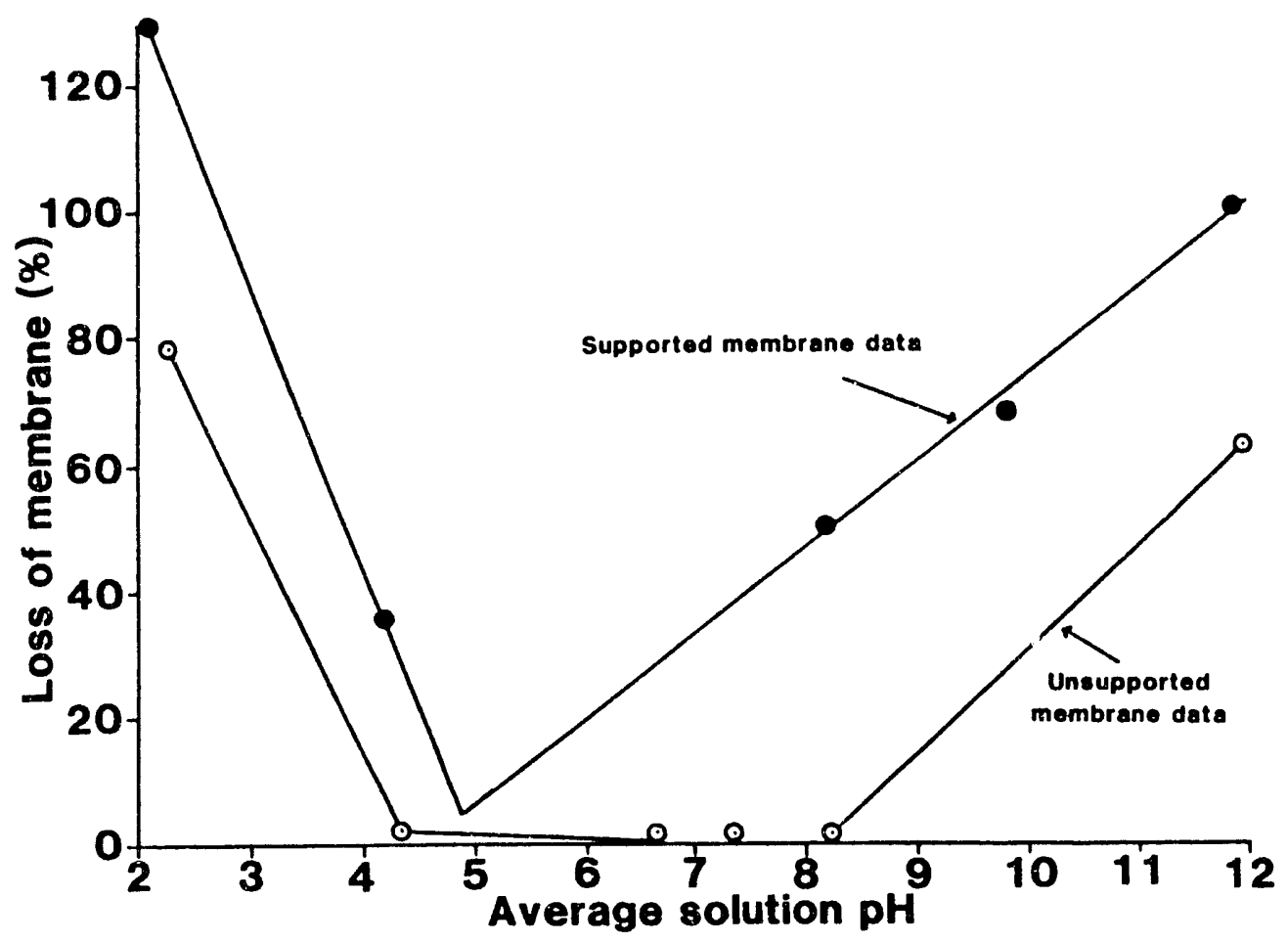

Figure 50. Loss of alumina membranes as a function of $\mathrm{pH}$ after a 10 week exposure to test solutions at various $\mathrm{pH}$ values.

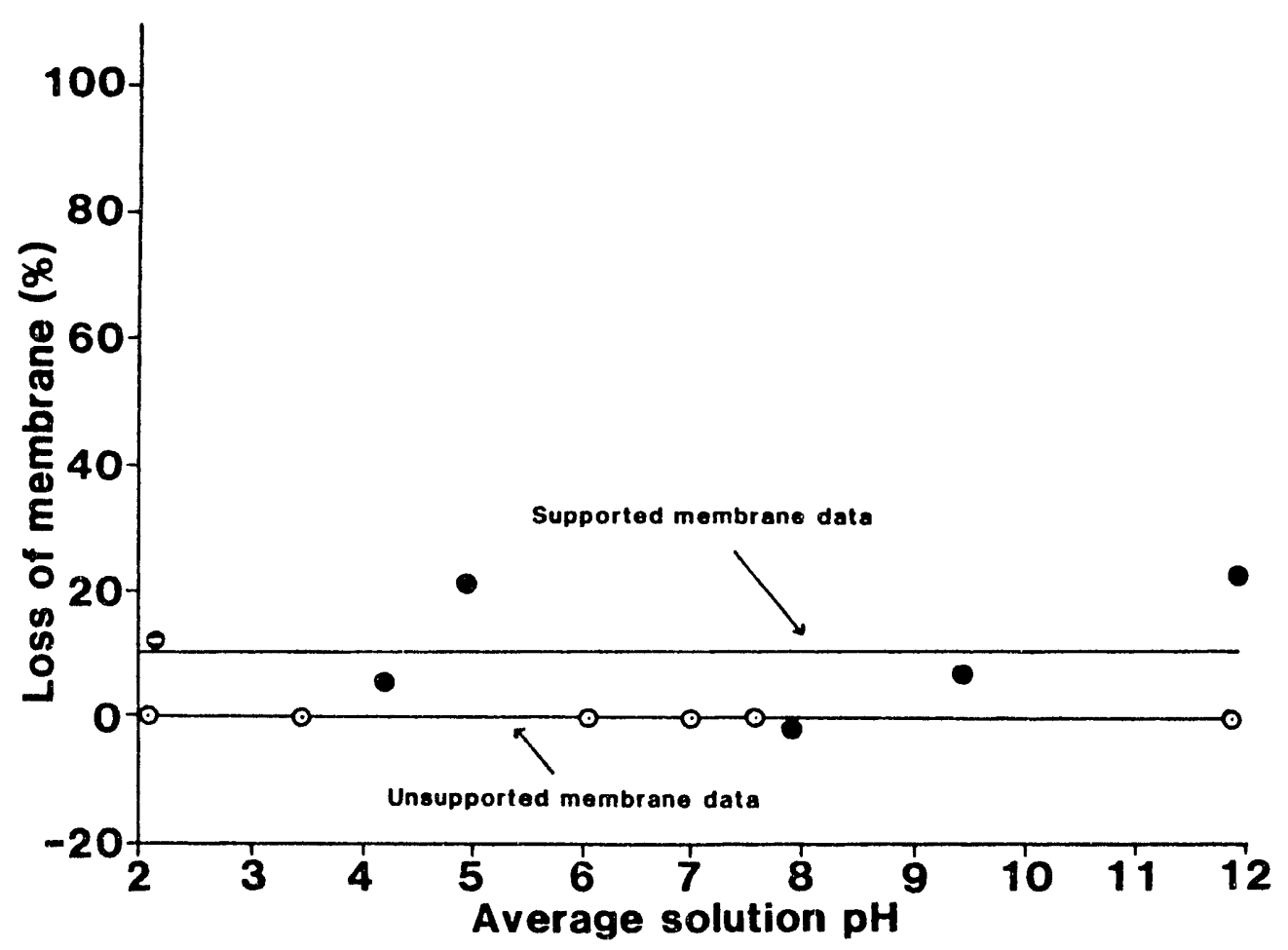

Figure 51. Loss of titania membranes as a function of $\mathrm{pH}$ after a 10 week exposure to test solutions at various $\mathrm{pH}$ values. 
the test solution was greater than the flux through the original support before it was dipped into the boehmite sol. These results indicate that both the membrane and the underlying clay support were affected by the $\mathrm{pH}$.

However, the dissolution experiments on the clay supports themselves did not support this view because the clay supports were also very stable. The largest measured loss was 4.88 of the aluminum in the clay support at $\mathrm{pH} 11.9$. All other analyses for aluminum, titanium and iron indicated losses no greater than 1.18. Because an analysis for silicon could not be performed, it is possible that degradation of the supports was occurring in these systems. However, the membrane losses for titania which are shown in Figure 51 indicate that the clay supports had little effect on these systems. No loss of titanium was observed for unsupported titania membranes. The losses for the supported membrane systems appear to be randomly scattered about a $10 \%$ loss. Thus, it appears that some of the loss of the supported alumina membrane may be attributable to errors in the permeability technique, even though the drying procedure described earlier for the supported membranes was designed to minimize these errors. It is not clear that these large losses of the supported alumina membrane indicate that further problems exist with the permeability measurement technique. Another possibility is that the membrane and support are separating from one another during these experiments.

\section{Conclusions}

Both clay supports and titania membrane systems showed little effect of a 10 week exposure to test solutions maintained between $\mathrm{pH}$ values of 2 and 12 . Alumina membrane systems, however, did degrade, although unsupported alumina 
membranes held between $\mathrm{pH} 4$ and 8 appeared to be quite stable. At $\mathrm{pH} 2$ and 12 , these membranes suffered severe material losses over a 10 week period due to dissolution of the $\gamma$-alumina of which these membranes are comprised. The less soluble $\alpha$-alumina, which should be more resistant to acids and bases, would be obtained by firing at higher temperatures. However, membranes composed of $\alpha$ alumina would be expected to have larger average pore sizes, so this approach to obtaining better $\mathrm{pH}$ stability may be practical only in special situations. Otherwise, alumina membranes should be exposed to strongly acidic and alkaline conditions only for short periods of time. This situation precludes the use of such membranes in processing highly acidic or highly alkaline feedstocks. Alumina membranes could still be used in situations where only occasional cleaning with such corrosive solutions would be required to remove foulants. A comparison of permeability measurements on supported alumina and titania membranes showed significantly higher losses of membrane material from the alumina membranes under all pH conditions. These higher losses must be related to the relatively high solubility of the $\gamma$-alumina which comprises the membrane. However, other factors also affect the measurement of membrane loss, so that a full explanation cannot be given at present.

\section{b. Changes in Permeate Flux with $\mathrm{pH}$}

When hydrous oxide particles are suspended in aqueous solutions, they develop a charge that depends on the $\mathrm{pH}$ and ionic strength of the solution $[18,19]$. Similar behavior would be expected for oxide particles that are fabricated into a supported membrane. If the pores of this membrane are sufficiently small that the electric fields produced by the charged particles cover 
the entire pore volume, then one would expect the rate of transport of aqueous solutions through the charged membrane to be affected. In particular, the permeate and/or solute fluxes would vary with $\mathrm{pH}$. The largest fluxes would be as sociated with conditions that cause the particles to bear very little charge. Such effects were observed when the permeation rates of $0.01 \mathrm{M} \mathrm{NaClO}_{4}$ test solutions through supported alumina and titania membranes were measured (see Figures 52 and 53). (The two curves shown in each figure result from analyzing the permeate flux data in two different ways.) However, another consideration complicates the interpretation of the data shown in these figures. In this experiment, the supported membranes were dry when they were brought into contact with the aqueous electrolyte. Once a dry oxide membrane is placed in water, hydration reactions will occur between the oxide and water. This wetting of the oxide surface will occur at any $\mathrm{pH}$, although the kinetics of hydration would be expected to be $\mathrm{pH}$ dependent. Thus, changes in flow rates would be expected to occur due solely to the hydration of the oxide membrane.

A second experiment was performed in which attempts were made to minimize the effects of hydration. In this experiment, supported membranes were stored in the electrolyte solution at $\mathrm{pH} 6.3$ for 24 hours before use. These membranes were then flushed with the same electrolyte solution until the flow rate had stabilized. After this, the $\mathrm{pH}$ was changed and the process repeated. Flow rates in this system were still observed to vary with $\mathrm{pH}$, even though the effects of hydration had been minimized.

Results from both experiments indicate that the largest permeabilities were obtained at $\mathrm{pH}$ values near those which should correspond to minimally charged surfaces ( $\mathrm{pH} 5$ to 6 for titania; $\mathrm{pH} 8$ to 9 for alumina). At $\mathrm{pH}$ values 


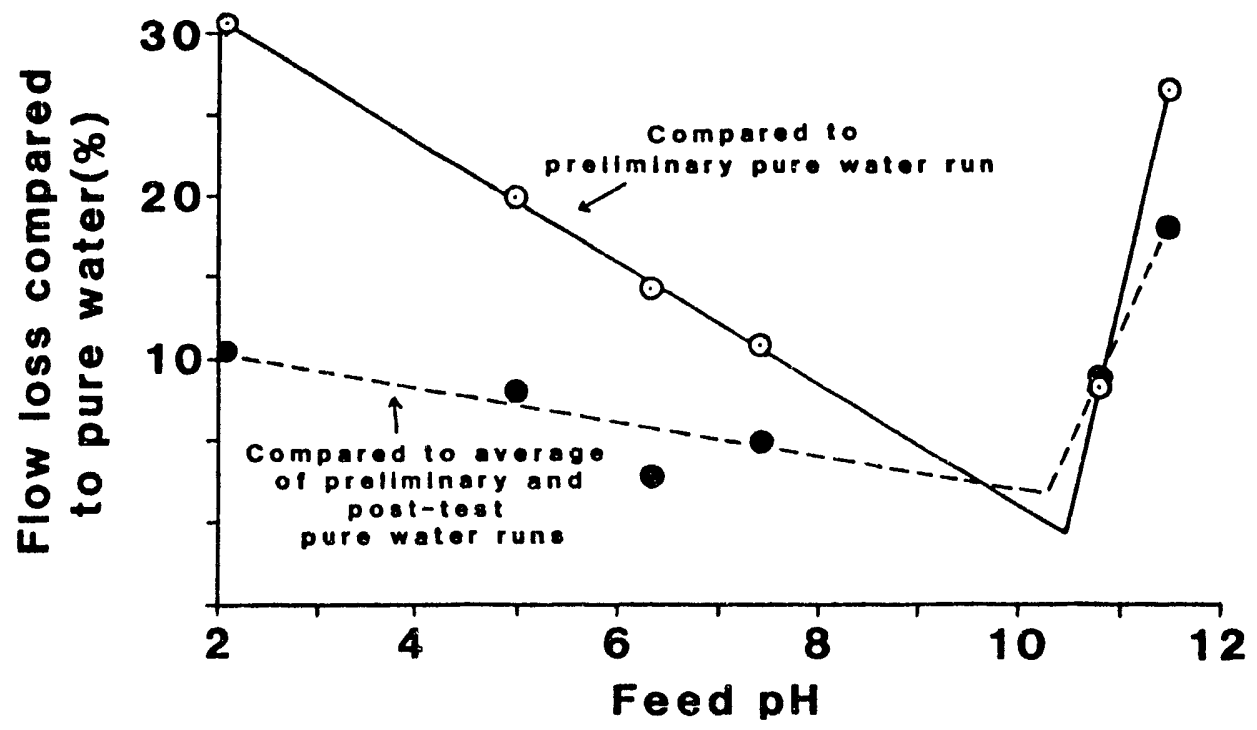

Figure 52. Effect of feed $\mathrm{pH}$ on the loss of flow of a $0.01 \mathrm{M} \mathrm{NaClO}_{4}$ test solution through a supported alumina membrane.

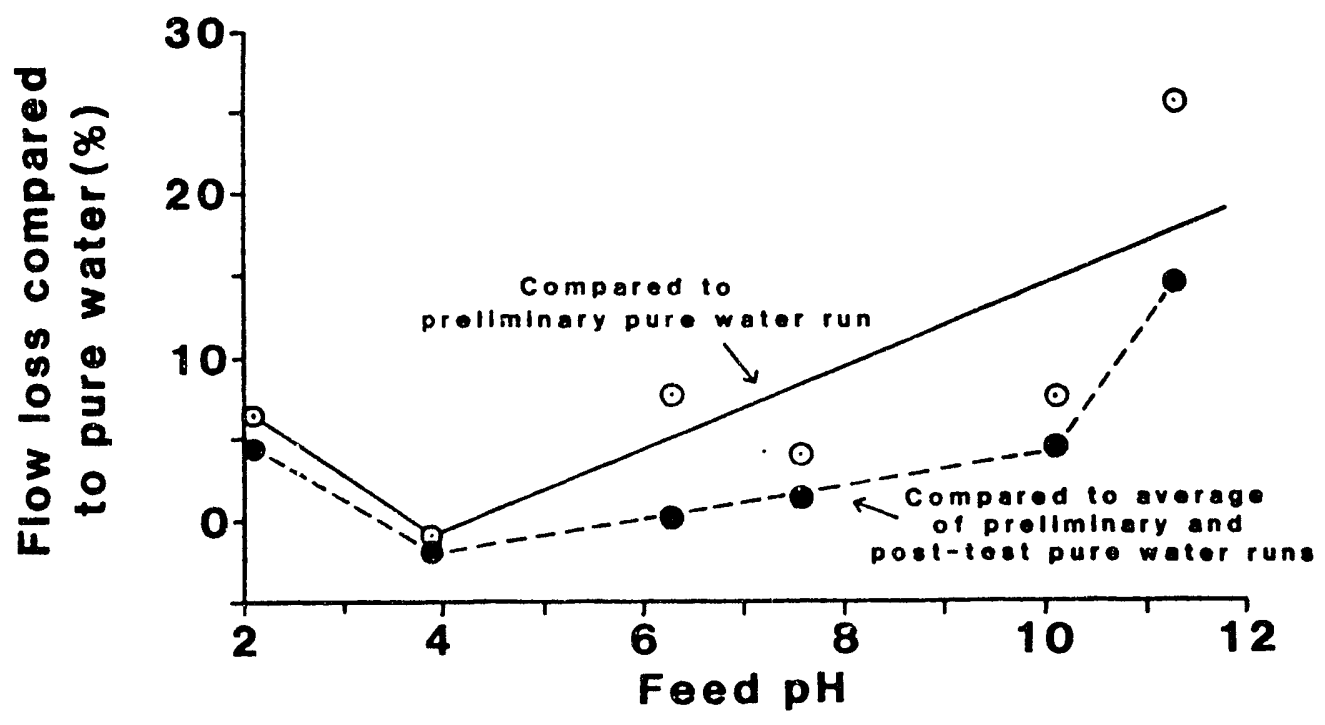

Figure 53. Effect of feed $\mathrm{pH}$ on the loss of flow of a $0.01 \mathrm{M} \mathrm{NaClO}_{4}$ test solution through a supported titania membrane. 
far from these, the surfaces should be more highly charged and the permeation rates of the electrolyte solutions should decrease, a result observed experimentally. We expect that these effects will be more important for smaller sized pores, but less important for electrolyte solutions with higher ionic strengths. It is also interesting to note that the flow rates were more affected by high $\mathrm{pH}$ values than by low $\mathrm{pH}$ values. This result may indicate that the more highly hydrated cations are being attracted to the negatively charged oxide surfaces at high $\mathrm{pH}$, thus impeding water flow. Less hydrated anions attracted to positively charged surfaces at low $\mathrm{pH}$ have less effect on the overall flux.

4. Time of Exposure to Test Stream

As mentioned earlier, oxide ceramic membranes which are exposed to aqueous solutions should be affected by the $\mathrm{pH}$ of those solutions. Once this effect had been verified experimentally, it was important to consider how the kinetics of hydration of these oxide membranes cotild change membrane permeabilities. Supported alumina and titania membranes were prepared and maintained in a dry condition prior to testing. Fluxes of water through these membranes were then measured periodically for several days, while storing the membranes in deionized water between measurements. Consequently, only initial flux measurements were performed on dry membranes.

The permeation rates of pure water through these membranes were observed to decline as time elapsed, but the loss in permeate flux was much greater when flows were being measured than when the membranes were stored in water. Thus, membrane hydration was much more efficient when water was being forced 
through the membranes than when stagnant water was contacting the membranes. Figure 54 contains data for the loss in the permeation rate of water as a function of $t$ :e elapsed time for which each type of membrane was exposed to flowing water. For both membranes the flux loss was about 3.38 of the original flow per hour over the initial 10 to 12 hours on stream. After this initial loss, the decay in flux dropped to 0.48 and 1.48 per hour for the $\mathrm{Al}_{2} \mathrm{O}_{3}$ and $\mathrm{TiO}_{2}$ mumbranes, respectively. Subsequent tests proved that the clay supports and the water temperature had negligible effects on the measured declines in flux.

Although menbrane hydration may have affected the permeabilities measured in other tests in this project, it is unlikely that significant errors have been introduced. Unless otherwise noted, all data were collected on mem-

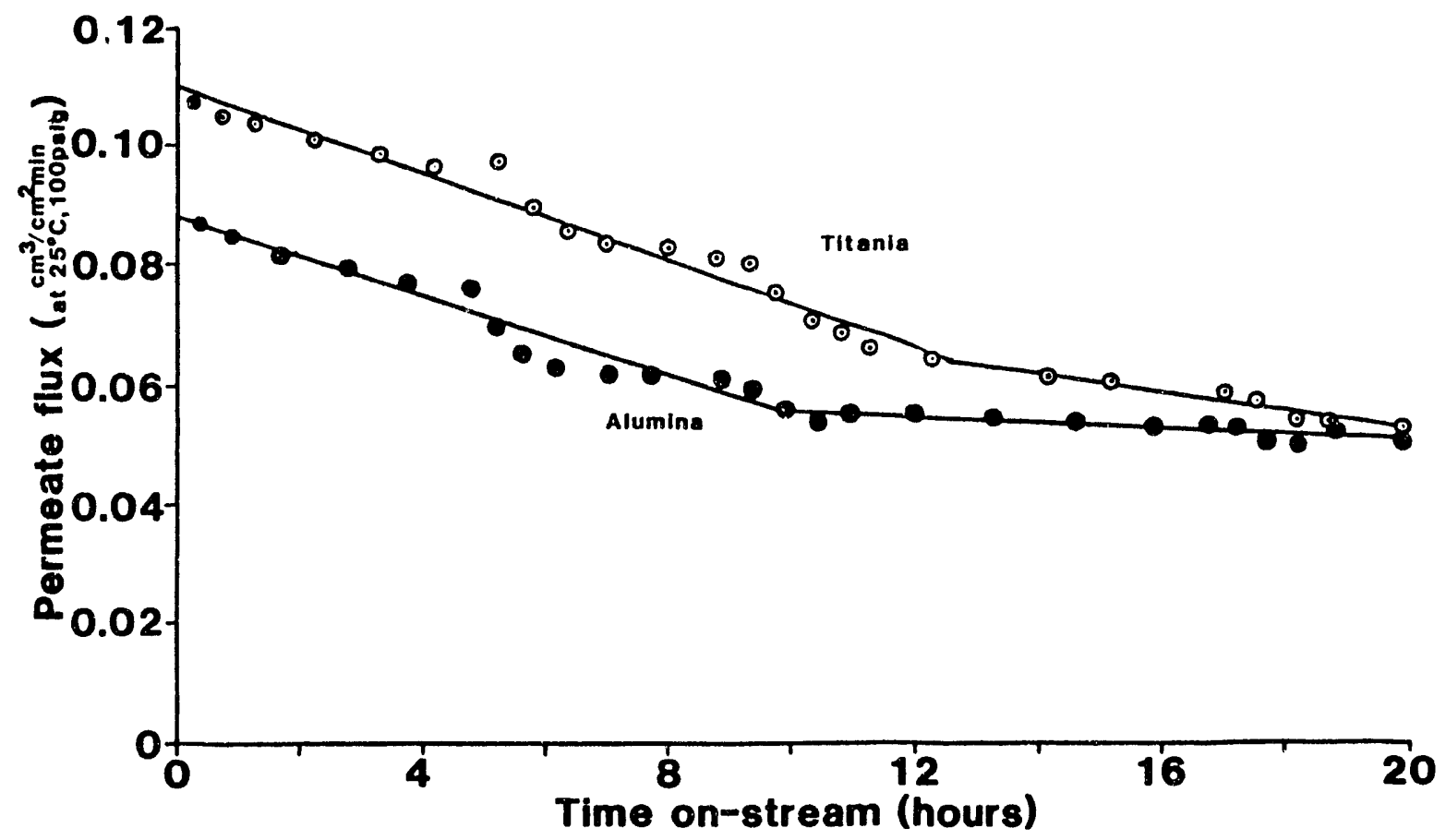

Figure 54. Effect of the contact time between supported membranes and water on the permeation rate of water through these membranes. 
branes which were fired and dry at the start of each run. Flow measurements were initiated as soon as the flows were stable, typically within $10 \mathrm{~min}$ of commencing pumping. These precautions minimized any variations in recorded permeability that might have resulted from differing degrees of hydration.

Although permeate flux measurements should not have been compromised by using different hydration times for the supported membranes, permselectivity studies were affected. When trying to reproduce some of our earlier results on the rejection of polyethylene glycol test solutions (molecular weight = 1000), we noticed a much poorer rejection rate (ca. 508 as compared to ca. 908 achieved earlier for similar membrane systems). After investigating this discrepancy for several months, we finally discovered that the rejections could be significantly enhanced by simply wetting the membranes one week before use.

Results of these hydration studies on several similar supports are given in Table XVIII. The best rejections (80-908) were obtained with supports that had been immersed in deionized water for 15 days immediately prior to use. The poor results obtained with dry systems could be reproduced by simply firing the hydrated supported membranes at $350^{\circ} \mathrm{C}$ for $0.5 \mathrm{~h}$ and then performing the rejection study without allowing sufficient time for the membranes to rehydrate. If these same membranes were fired and then equilibrated with water for 15 days it was possible to restore their performance to the higher level. It should be noted that the 15 day hydration period was arbitrary and probably could be shortened considerably.

These results show that the membranes can be successfully regenerated but they must be reequilibrated with water after regeneration. Ceramic membranes fabricated from oxides will require a hydration period before being brought onstream in commercial applications. 
Table XVIII. Effects of hydration and dehydration on the permeate fluxes and permselectivities of seven different supported membranes at different stages of regeneration and hydration of the membranes.

Treatment Permeate Flux Rej Perm Flux Rej Perm Flux Rej Cycles Water PEG Soln (8) for PEG Soln ( 8 ) for PEG Soln (8)

$\begin{array}{lccccccccc}5 & 0.094 & 0.025 & 62 & \text { R H } & 0.008 & 92 & \text { R } & 0.009 & 65 \\ 5 & 0.129 & 0.025 & 57 & \text { R H } & 0.011 & 90 & \text { R } & 0.010 & 63 \\ 5 & 0.141 & 0.030 & 49 & \text { R H } & 0.009 & 88 & & & \\ 5 & 0.149 & 0.029 & 56 & \text { R H } & 0.010 & 80 & & & \\ 4 & 0.140 & 0.027 & 56 & \text { R H } & 0.009 & 88 & \text { R } & 0.012 & 66 \\ 4 & 0.132 & 0.024 & 65 & \text { R H } & 0.010 & 84 & \text { R } & 0.013 & 61 \\ 4 & 0.128 & 0.023 & 50 & \text { R H } & \ldots & 78 & \text { R } & 0.010 & 61\end{array}$

Permeate fluxes were measured in units of $\mathrm{cm}^{3} / \mathrm{cm}^{2} / \mathrm{min}$ at $25^{\circ} \mathrm{C}$.

$R$ represents a regeneration step in which the supported membrane was heated to $350^{\circ} \mathrm{C}$ at a ramp rate of $1.7^{\circ} \mathrm{C} / \mathrm{min}$, held at $350^{\circ} \mathrm{C}$ for $0.5 \mathrm{~h}$, and cooled to $85^{\circ} \mathrm{C}$ at a ramp rate of $1.7^{\circ} \mathrm{C} / \mathrm{min}$.

$\mathrm{H}$ represents a hydration step in which the supported membrane was placed in deionized water for 15 days.

Each treatment cycle was 4 dippings in an alumina sol followed by one firing.

The support was withdrawn from the sol at $16.7 \mathrm{~cm} / \mathrm{min}$.

\section{Temperature of the Feed Stream}

The rates at which various species are transported through permselective polymeric membranes have been shown to exhibit an Arrhenius temperature dependence $[28,29]$ :

$$
J=J_{0} \exp \left(-E_{a} /(R T)\right)
$$

where $\mathrm{J}$ is the measured permeate flux. If this relation is obeyed, a plot of In $\mathrm{J}$ versus $1 / \mathrm{T}$ will be linear.

To determine if this type of behavior is also characteristic of ceramic membranes, the permeation rates of pure water through supported alumina and titania membranes were measured at temperatures between 7 and $40^{\circ} \mathrm{C}$. Figure 55 
indicates that Arrhenius behavior was observed over this temperature range, although the high temperature data appeared to fall slightly below the expected values. This discrepanzy was probably due to vaporization and recondensation of permeate onto the permeate outlet line on the permeability test apparatus .

Activation energies of 4.0 and $3.6 \mathrm{kcal} / \mathrm{mole}$ for the transport of water through alumina and titania supported membranes, respectively, can be calculated from the slopes of these graphs. These values were similar to, but slightly lower than, those observed for polymeric membranes (typically about 5 $\mathrm{kcal} / \mathrm{mole}(28,29\})$.

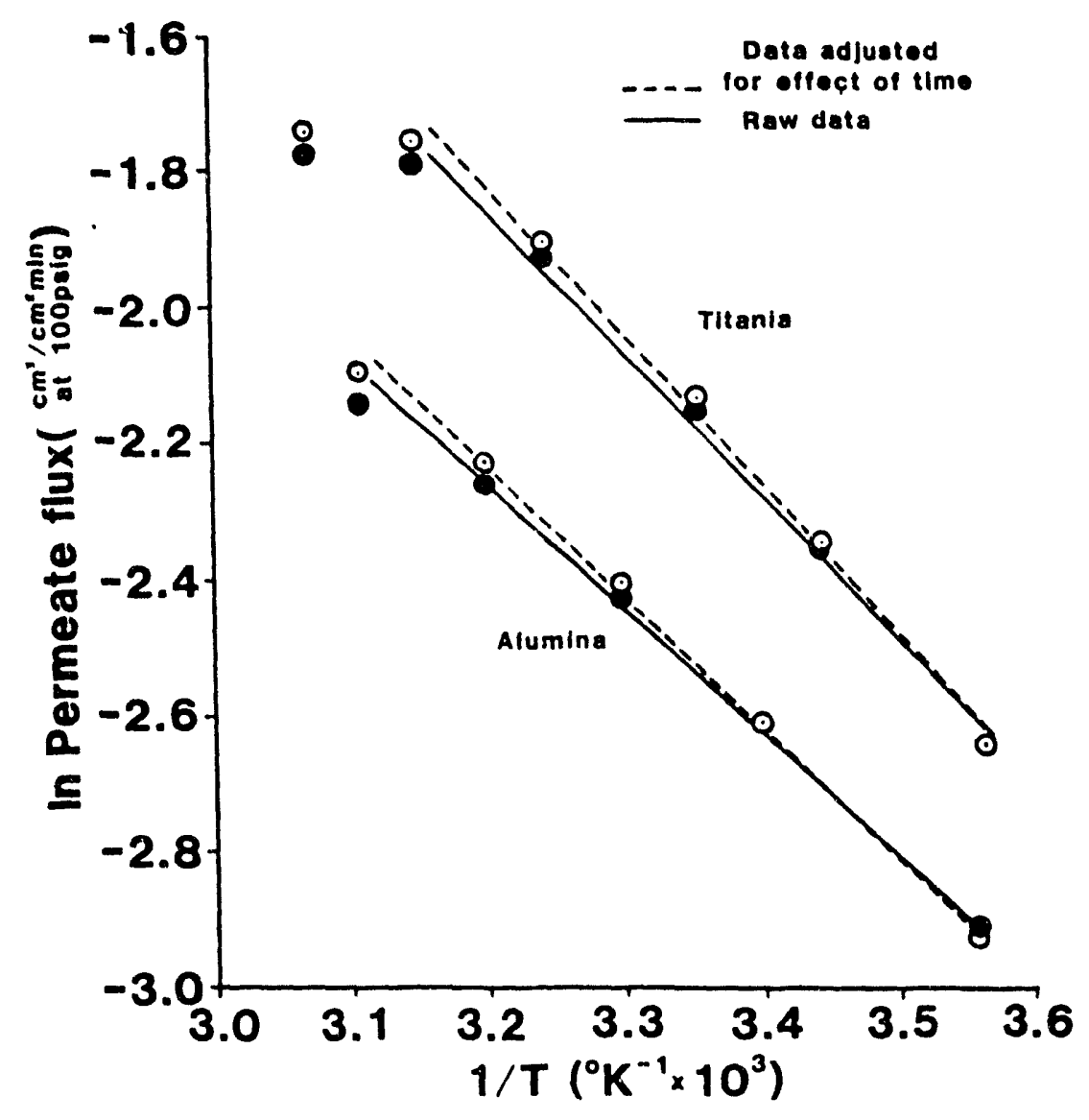

Figure 5j. Variation of permeate fll $x$ with temperature. 
6. Pressure of the Feed Stream

Figure 56 shows the effect of the operating pressure of the system on the permeation rate of distilled water through supported alumina membranes. For mechanically stable systems, it is usually assumed that permeability is independent of pressure. As indicated by this nlot, the permeabilities of these alumina membranes were slightly dependent on pressure, as the permeabilities increased with increasing pressure.

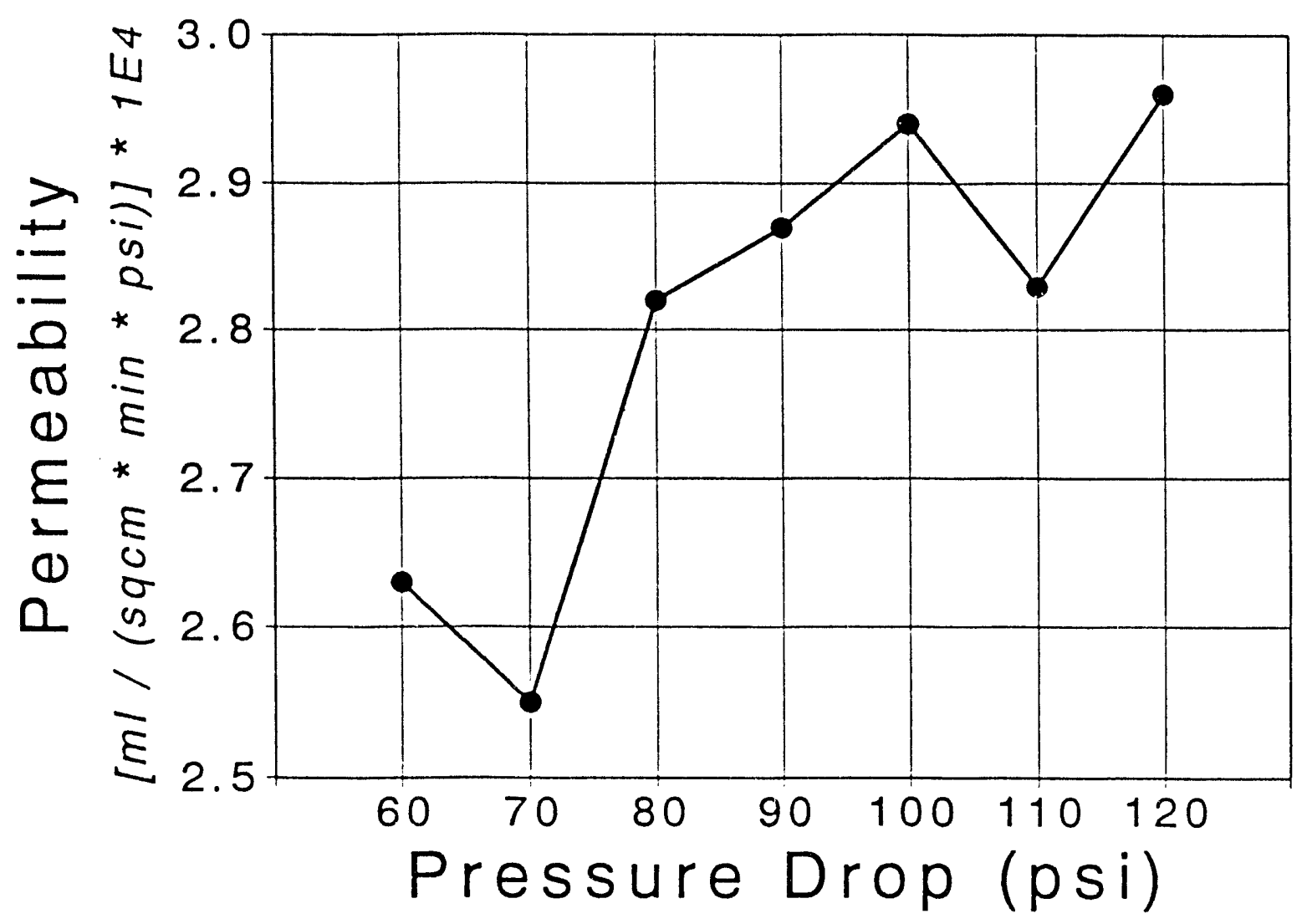

Figure 56. Permeability at $25^{\circ} \mathrm{C}$ for supported alumina membranes as a function of the pressure drop across the membrane. 
B. Permselectivity Measurements

1. Procedures Employed

The refection characteristics of supported alumina and titania ceramic membranes have been determined for several different aqueous test streams: polyethylene glycol (PEG, $18 \mathrm{w} / \mathrm{w}$ ) at several different average molecular weights from 200 to 8,000 , bovine serum albumin (nominal molecular weight of $66,000)$, cheese whey proteins and cheese whey total solids. Total organic carbon analyses were performed to determine the concentrations of PEG and bovine serum albumin solutions. Analyses of the solutions of cheese whey proteins for total nitrogen and non-protein nitrogen were carried out using the Kjeldahl method. The total solids content of each cheese whey process stream was determined by the gravimetric method of the AOAC. The test 1oop was operated with total recycle of retentate and permeate streams so as to maintain a constant composition of the feed stream. Solute rejections were calculated as

Percent Rejection $=100\left(1-\left[C_{\text {permeate }} / C_{\text {feed }}\right]\right)$

In general, the membranes were coated on clay supports and were fabricated using the protocol described on p. 108. These supported membranes typically were fired after every 4 dips. This sequence was repeated as needed to obtain the desired number of coatings on the support. 


\section{Permselectivities of Supported Alumina Membranes}

Figure 57 shows the rejection data obtained for PEG solutions of various molecular weights in contact with supported alumina membranes as a function of the number of coatings of the alumina $[2,5]$. As the molecular weight of the PEG increased, the supported alumina membranes exhibited an increase in rejection, as would be expected for larger molecules. However, because the values of these rejections were surprisingly high, the study was repeated. This second study resulted in the finding that the time of hydration affected the permselectivity of the supported membrane, as discussed in Section VI.A.4. Long hydration times resulted in high rejection coefficients [3]. Further studies have shown somewhat lower rejections than those suggested in Figure

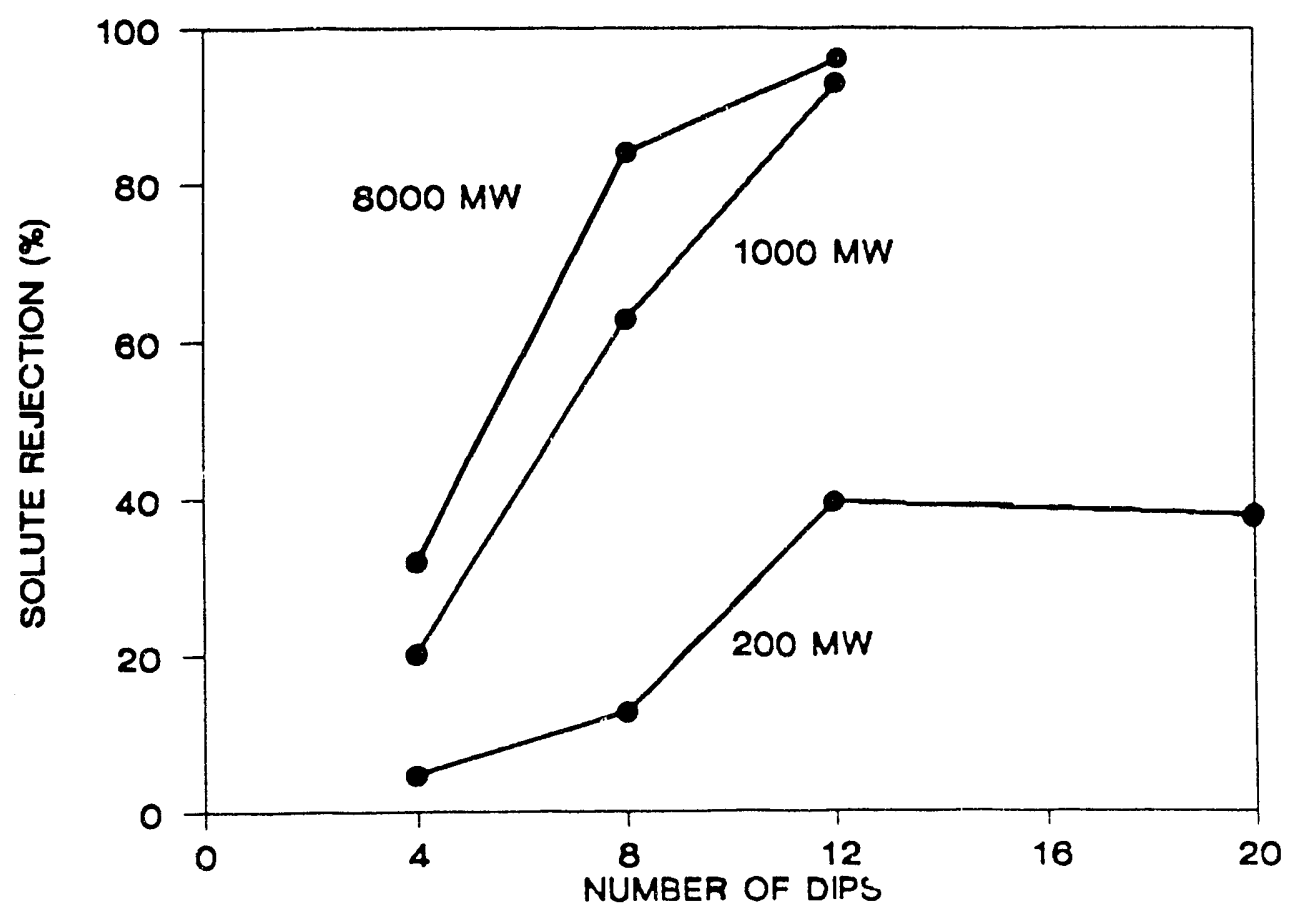

Figure 57. Rejection of polyethylene glycols by supported alumina membranes. 
57, but we still find a nominal molecular weight cutoff of ca. 2,000 (defined as that corresponding to $80 \%$ rejection) for alumina membranes coated 12 times on our porous clay supports [25]. Also note that, as shown in Figure 57, no benefit in the rejection characteristics was obtained by applying more than 12 coatings of the alumina sol to a porous clay support [2].

Figure 58 displays permselectivity data for several materials filtered through alumina membranes that were coated 12 times on clay supports. The data for cheese whey proteins and cheese whey total solids were obtained from an acid cheese whey sample provided by the University of Wisconsin dairy plant. This sample had been produced during their manufacture of cottage cheese. Permselectivity measurements for the cheese whey total solids were complicated by the excessive fouling of the membranes that occurred when this

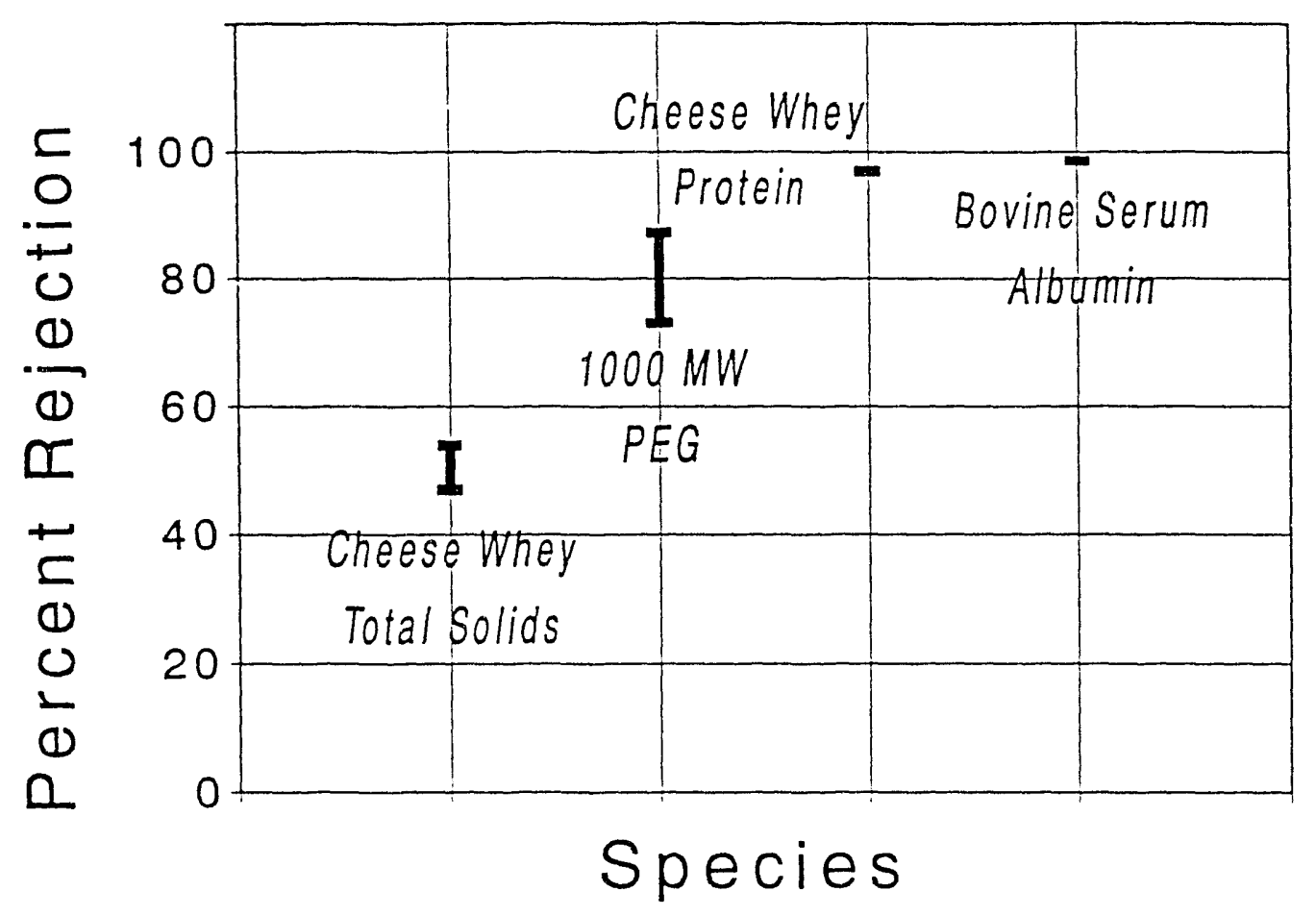

Figure 58. Rejection of several species by supported alumina membranes. 
material was used at room temperature. We attributed this fouling to inadequate clarification of the whey prior to use. Our attempts to regenerate these fouled membranes are described in Section VI.C.

In spite of the problems in using cheese whey total solids, the supported alumina membranes gave essentially 1008 rejection of bovine serum albumin and cheese whey proteins, within analytical error. These high rejections were observed even though the permeabilities of the membranes to these feed streams were only $25-358$ of their permeabilities for pure water. Supported alumina membranes did show much poorer rejections (ca 508) for cheese whey total solids. This result is a consequence of the permeability of the membranes to the low molecular weight solutes present in cheese whey (e.g., lactose and inorganic salts) $[2,25]$.

The performance of our supported alumina membranes in permselectivity studies was compared to the performance of supported alumina and zirconia membranes being developed by Alcoa. In all cases, aqueous solutions of polyethylene glycol polymers of different molecular weights were used as test solutions. Inspection of the data presented in Figure 59 indicates that the rejection characteristics of our alumina membrane system were clearly superior to those of the systems available from Alcoa for applications involving solutes at the low molecular weight end of the ultrafiltration spectrum.

3. Effects of Phosphate Treatment on the Properties of Supported Alumina Membranes

Our initial permselactivity studies were performed with alumina membranes that contained added phosphate. Several studies were later performed 


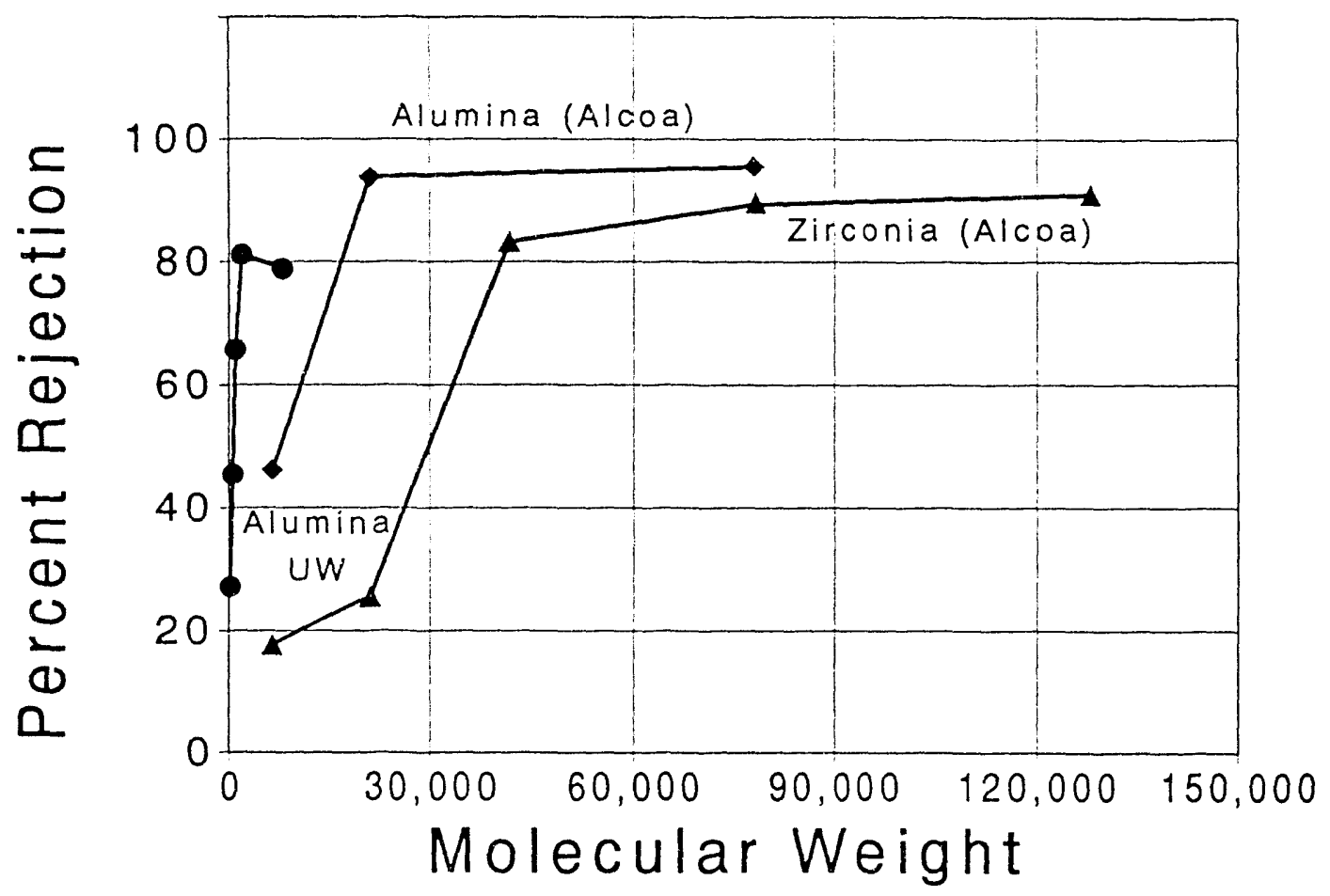

Figure 59. Comparison of the permselectivities of our supported alumina membranes and two inorganic membranes being developed by Alcoa.

to determine the effect of adding different levels of phosphate on the properties of both the particles in the alumina sols and the supported membranes themselves. The effects of adding different amounts of phosphate on the properties of unsupported alumina and titania membranes were described in Sections IV.A.1.d and IV.A.3.f, respectively. All additions of phosphate to sols described in this section were performed at the "early" stage of sol preparation, using the same definition of "early" as given in Section IV.A.1.d. (These experiments were performed before we understood the importance of several factors (e.g., amount of hydration) on the performance of these supported membranes. Consequently, the behavior of these systems was not optimized. These data are presented here because they provide further information about the effect of phosphate on the performance of supported alumina membranes.) 
The alumina sols were characterized by measuring their $\mathrm{pH}$ and the electrophoretic mobilities of the constituent particles. Supported membranes prepared from these sols were characterized by determining their permselective properties. All supports were dip coated using hand withdrawal. After each dipping, the coated supports were allowed to dry in air. After five repetitions of this dipping and drying cycle, the coated supports were heated at $1.7^{\circ} \mathrm{C} / \mathrm{min}$ to $500^{\circ} \mathrm{C}$ and held at that temperature for $1 \mathrm{~h}$. This sequence of coating, drying and firing was then repeated. Measurements of the permeation rate of deionized water through a given support were performed before the support was coated.

The data in Tables XIX through XXII illustrate how the properties of the sols and the supported membranes changed with time. The $\mathrm{Al}_{2} \mathrm{O}_{3}$ sols containing phosphate seemed to be stable over time, since the particle mobility and $\mathrm{pH}$ did not change significantly even after several months. In several experiments, supports were coated with sols aged for 8 days, 3 weeks, 1 month, and 3 months. Membranes formed from these sols were then tested for the rejection of PEG (nominal molecular weight of 1000). Rejections were better for membranes derived from sols aged for one month and three months. Supports

Table XIX. Effects of phosphate on properties of supported membranes prepared from boehmite sols aged for 8 days. (Permeate fluxes were measured in $\mathrm{cm}^{3}$ of solvent $/ \mathrm{cm}^{2}$ of membrane/min and mobilities in $10^{-8} \mathrm{~m}^{2} / \mathrm{V}$-sec.)

\begin{tabular}{rrrrrr}
$\begin{array}{c}\text { Phosphate } \\
\text { Conc. (M) }\end{array}$ & Mobility & $\mathrm{pH}$ & $\begin{array}{c}\text { Permeate } \\
\text { Pure Water }\end{array}$ & PEG folution & $\begin{array}{c}\text { Rejection } \\
(8)\end{array}$ \\
\hline & & & & & \\
$10^{-5}$ & +4.12 & 4.1 & 0.114 & 0.046 & 32 \\
$2 \times 10^{-5}$ & +4.11 & 4.2 & 0.116 & 0.040 & 13 \\
$10^{-4}$ & +3.82 & 4.1 & 0.118 & 0.033 & 17 \\
$10^{-3}$ & +3.96 & 4.1 & 0.119 & 0.053 & 7
\end{tabular}


Table XX. Effects of phosphate on properties of supported membranes prepared from boehmite sols aged for 3 weeks. (Permeate fluxes were measured in $\mathrm{cm}^{3}$ of solvent $/ \mathrm{cm}^{2}$ of membrane/min.)

$\begin{array}{cccc}\begin{array}{c}\text { Phosphate Conc } \\ (\mathrm{M})\end{array} & \begin{array}{c}\text { Permeate Flux for } \\ \text { Pure Water }\end{array} & \begin{array}{c}\text { Rejection } \\ \text { PEG Solution }\end{array} & \begin{array}{c}(8) \\ 0.5 \times 10^{-5}\end{array} \\ 0.5 \times 10^{-5} & 0.054 & 0.021 & 14 \\ 2 \times 10^{-5} & 0.051 & 0.025 & 12 \\ 10^{-4} & 0.048 & 0.024 & 22 \\ 10^{-4} & 0.053 & 0.021 & 34 \\ 10^{-3} & 0.063 & 0.029 & 31 \\ & 0.081 & 0.031 & 35\end{array}$

Table XXI. Effects of phosphate on properties of supported membranes prepared from boehmite sols aged for 1 month. (Permeate fluxes were measured in $\mathrm{cm}^{3}$ of solvent $/ \mathrm{cm}^{2}$ of membrane/min and mobilities in $10^{-8} \mathrm{~m}^{2} / \mathrm{V}$-sec.)

\begin{tabular}{cccccc}
$\begin{array}{c}\text { Phosphate } \\
\text { Conc. (M) }\end{array}$ & Mobility & pH & \multicolumn{2}{c}{$\begin{array}{c}\text { Permeate Flux for } \\
\text { Pure Water }\end{array}$} & $\begin{array}{c}\text { Rejection } \\
\text { PEG Solution }\end{array}$ \\
\hline & & & & & \\
$10^{-5}$ & +4.07 & 4.1 & 0.048 & 0.021 & 47 \\
$10^{-5}$ & +4.07 & 4.1 & 0.043 & 0.020 & 48 \\
$10^{-5}$ & +4.07 & 4.1 & 0.047 & 0.015 & 41 \\
$2 \times 10^{-5}$ & +4.07 & 4.2 & 0.115 & 0.024 & 52 \\
$10^{-4}$ & +4.22 & - & 0.173 & 0.070 & 14 \\
$10^{-4}$ & +4.22 & - & - & - & 18 \\
$10^{-3}$ & +3.93 & 4.1 & 0.051 & 0.01 & 59
\end{tabular}

Table XXII. Effects of phosphate on properties of supported membranes prepared from boehmite sols aged for 3 months. (Permeate fluxes were measured in $\mathrm{cm}^{3}$ of solvent $/ \mathrm{cm}^{2}$ of membrane/min.)

\begin{tabular}{cccc}
$\begin{array}{c}\text { Phosphate Conc } \\
(\mathrm{M})\end{array}$ & $\begin{array}{c}\text { Permeate Flux for } \\
\text { Pure Water }\end{array}$ & $\begin{array}{c}\text { Rejection } \\
(8)\end{array}$ \\
\hline & & & \\
$0.5 \times 10^{-5}$ & 0.055 & 0.029 & 28 \\
$0.5 \times 10^{-5}$ & 0.066 & 0.026 & 32 \\
$2 \times 10^{-5}$ & 0.056 & 0.016 & 50 \\
$2 \times 10^{-5}$ & 0.047 & 0.026 & 32 \\
$10^{-4}$ & 0.048 & 0.023 & 50 \\
$10^{-4}$ & 0.062 & 0.024 & 47 \\
$10^{-3}$ & 0.074 & 0.024 & 44
\end{tabular}


coated with a one month old sol containing $10^{-5} \mathrm{M}$ phosphate gave relatively good rejections (ca. 508) which increased when these supported membranes were hydrated prior to testing. Mobilities were not always measured because they did not change appreciably during these experiments.

Three important issues should be noted when interpreting the results of these studies: (1) these supported membranes were not previously hydrated before being used in these studies; (2) the supported membranes were hand dipped; and (3) the amounts of phosphate added were not large enough to significantly affect the properties of either the boehmite particles in the sols or the membranes prepared from these sols. After we performed this study, we were quite concerned about the generally low and quite variable rejection coefficients measured in this study as compared to the high rejection coefficients measured earlier (ca. 908 for identical feed streams and supposedly identical supported membranes (see Section VI.B.2 and Figure 57)). Our attempts to reconcile these conflicting observations led us to realize the importance of the first two factors listed above in obtaining good permselectivities for these systems.

Based on the data obtained in this study, one would conclude that adding phosphate to alumina sols had no appreciable effect on the properties of the resulting supported membranes. Further permselectivity studies performed with supported alumina membranes reaffirmed this conclusion. (The data presented in Figure 59 and labeled "Alumina UW" was obtained with supported alumina membranes that contained no added phosphate.) While increasing the phosphate loading beyond the levels employed in this study might affect the properties of the resulting membranes, this approach would also increase the chance that 
the alumina sols would be destabilized. In order to obtain good coatings of alumina on supports, stable sols must be used.

\section{Permselectivities of Supported Titania Membranes}

As part of our early efforts to characterize supported membranes, permselectivity studies were performed on supported titania membranes. These membranes showed essentially no permselectivity for PEG test solutions of molecular weight 8,000 . This result was attributed to the presence of cracks in the membrane. However, these same membranes did show rejection coefficients of 87-978 for solutions of bovine serum albumin with a nominal molecular weight of $66,000[2,5]$.

While no further permselectivity studies were performed with supported titania membranes, it should be noted that these membranes displayed very good $\mathrm{pH}$ stability characteristics, much better than the supported alumina membranes. Thus, supported titania membranes might prove useful for certain separations under $\mathrm{pH}$ conditions that would not be conducive to the use of supported alumina membranes. 


\section{Regeneration of Membranes}

A cleaning or regeneration procedure must be developed for these membranes before they can be employed commercially. To date, selected regeneration methods have been tested only for supported alumina membranes which have been fouled during studies with cheese whey.

The first method tested was to wash the fouled membranes with water and heat them to $500^{\circ} \mathrm{C}$ for 1 hour. After two such cleaning cycles, the permeability of the membranes to water dropped by 25 to $50 \%$. Despite this loss in permeability, the membranes were still able to reject in excess of 958 of the cheese whey protein [25].

However, alternative cleaning processes were studied with the goal of developing a method which would not require the use of extreme temperatures, yet would prevent any loss in the initial permeability of the membrane after each cleaning cycle. The method currently employed is to manually clean the membrane and then flush it with warm water for 0.5 hour after each use. Manual cleaning involves removing the membrane from the test apparatus and rinsing off the layer of foulant that has built up during the time of operation. When the utility of this regeneration process was tested for a period of 20 days, it was found that the initial permeability of the membrane could be consistently regenerated and that the rejection of cheese whey was still essentially 1008 at the end of this test period.

This cleaning process has been developed to meet the requirements of the geometry of our supported membranes and our test apparatus. By improving the design of this system, the loss of permeability of the membrane during a test run can be decreased because the concentration polarization layer which forms 
during the run can be limited. An improved design would also allow cleaning to be done in place by scouring the membrane surface. Moreover, the membrane could be steam sterilized as part of the regular plant sterilization procedure. While this expectation has not been tested as yet, we believe that our alumina membranes would exhibit behavior similar to the alumina membranes incorporated in the ceramic membrane systems that are sold commercially by Alcoa. Alcoa does subject their membranes to hydrothermal treatments that, we feel, correspond to steam treatments to at least some extent. 


\section{Conclusions}

\section{A. Supports}

Techniques were developed that allowed the fabrication of test-tube shaped, forous clay supports with reproducible permeabilities. These supports were then coated with either titania or alumina sols and used for studies of ultrafiltration separations. In these applications, the alumina-coated supports performed much better than comparable modules being developed by Alcoa. Because the pore sizes of these clay supports are in the range of microns, these supports are not being considered for use in preparing modules for studies of gas phase and reverse osmosis separations. For these latter studies, we are investigating the use of commercially available supports with pore sizes less than one micron.

\section{B. Unsupported Membranes}

\section{Techniques for Synthesizing Sols}

Protocols were developed for preparing metal oxide sols with particle diameters that cover the range from 2 to $200 \mathrm{~nm}$. Since larger particles (ca. 10 to $200 \mathrm{~nm}$ ) generally are aggregates of smaller primary particles, the sizes of these larger particles can be controlled by controlling the aggregation of the primary particles. Particle aggregation, in turn, depends on the $\mathrm{pH}$ and ionic strength of the suspension during and after hydrolysis of the precursor alkoxides. 
The classic approach to sol-gel processing is to hydrolyze the precursor alkoxide with water only. Since most oxides have isoelectric points within 1 to $2 \mathrm{pH}$ units of neutrality, the classic approach produces particles with little or no charge. These particles immediately aggregate in a relatively uncontrolled manner, so that peptization of the resulting aggregates is necessary in order to obtain stable sols. We have shown that the specific surface areas and porosities of unsupported titania membranes prepared from such sols are independent of the sizes of the particles in the sols and thus the amount of aggregation that has occurred.

In contrast, smaller particles (although still not in the size range characteristic of nanoparticles) can be produced by hydrolyzing the alkoxide at a $\mathrm{pH}$ value that is far from the isoelectric point of the final oxide. This approach results in the initial formation of particles with a large surface charge that electrostatically stabilizes the particles and prevents further aggregation. Aggregation of these charge stabilized sols can then be controlled by dialyzing the sols to a $\mathrm{pH}$ value that is closer to the isoelectric point and that produces a sol with the desired properties. We have shown that the properties of titania sols produced by acidic hydrolysis and dialyzed to different $\mathrm{pH}$ values affect, in turn, the specific surface areas, porosities and pore size distributions of unsupported titania membranes fabricated from those sols. Thus, this dialysis process provides much better control over the properties of ceramic membranes than the classic approach.

Two protocols were developed for synthesizing sols that contain nanoparticles (particles with diameters between 2 and $10 \mathrm{~nm}$ ). In one process, the precursor alkoxide is hydrolyzed under $\mathrm{pH}$ conditions that cause dissolution of any particles that form. This ionic solution is then dialyzed against deion- 
ized water. This process causes the oxide to reprecipitate, but under conditions that allow electrostatically stabilized nanoparticles to form. Note that metal salts, preferably nitrates, can be substituted for alkoxides in this synthetic scheme. The choice of a precursor material is mediated primarily by convenience. In the other process, the substituents of the precursor alkoxide are exchanged with tert-amyl alcohol to produce a tert-amyloxide. This sterically hindered alkoxide is then hydrolyzed with a limited amount of water using tert-amyl alcohol as the solvent. Under these conditions, hydrolysis occurs so slowly that nanoparticles are produced.

A major difference between the end products of these two methods of synthesizing nanoparticulate sols is the solvent employed. The former method produces aqueous sols, while the latter method requires the use of tert-amyl alcohol as the solvent. A second difference is that nanoparticles of mixed oxides may be somewhat easier to synthesize by the latter method. Nanoparticulate sols of $\mathrm{TiO}_{2}, \mathrm{ZrO}_{2}$ and $\mathrm{TiO}_{2}-\mathrm{ZrO}_{2}$ mixed oxides were prepared by both methods. A patent has issued that covers both methods of synthesizing nanoparticulate sols $[30]$.

\section{Effects of Adding Phosphate}

Incorporation of phosphate into mesoporous ceramic membranes did not significantly enhance either the structural or the permselective properties of those membranes. Phosphate was incorporated into membranes by adding either phosphoric acid or a dihydrogen phosphate salt to the precursor sols. However, the concentrations of phosphate that could be added to the precursor sols without destabilizing these sols were limited. Thus, only limited amounts 
of phosphate could be incorporated in the final membranes. These levels of phosphate may not have been large enough to have a noticeable effect on the properties of the resulting membranes.

However, there were indications that the incorporation of phosphate into supported membranes did affect the properties of those membranes to some extent. Figures 57 and 59 present results of two separate permselectivity studies that were performed using polyethylene glycol test solutions passed through alumina membranes slipported on porous clay supports. The data shown in Figure 57 was obtained using alumina membranes that contained phosphate, while the data shown in Figure 59 was obtained using alumina membranes without added phosphate. When the data in the two figures are compared, it appears that the phosphated alumina membranes exhibited somewhat better permselectivities to the test solutions than the nonphosphated alumina membranes. Because these tests were conducted two years apart, other factors may also have influenced these results.

We cannot state at this time that the incorporation of phosphate into mesoporous ceramic membranes has no beneficial effect on the structural and permselective properties of those membranes. Thus, we may perform further studies related to this question at some future date. Note, however, that the conditions necessary for phosphate addition to be beneficial may cover only a fairly narrow range of phosphate concentrations, $\mathrm{pH}$ values and solids concentrations. Since these conditions would also be dependent on the solid comprising the membrane, the determination of these conditions for each solid is likely to be a difficult problem. 
3. Thermal Stability of Membranes

A possible application for ceramic membranes prepared from nanoparticulate sols is for separations that are carried out in high temperature gas phase reactors. The ability of these membranes to withstand high temperatures without undergoing deleterious changes in their pore structures is an important concern for such an application. Unsupported membranes fabricated from nanoparticulate $\mathrm{TiO}_{2}$ and $\mathrm{ZrO}_{2}$ ools displayed limited thermal stabilities; the pore diameters of these materials underwent a significant increase when they were fired to $300^{\circ} \mathrm{C}$. However, unsupported membranes fabricated from mixed $\mathrm{TiO}_{2}-\mathrm{ZrO}_{2}$ nanopar culate sols displayed thermal stabilities that were enhanced by up to $200^{\circ} \mathrm{C}$ over the stabilities of the pure oxide membranes. The pore diameter of a membrane composed of $808 \mathrm{TiO}_{2}-208 \mathrm{ZrO}_{2}$ did not increase until it was fired above $500^{\circ} \mathrm{C}$. In general, we observed that mixed oxide membranes had enhanced thermal stabilities when compared to membranes composed of the corresponding pure oxides.

C. Fabrication and Properties of Unsupported Membranes

Modules were prepared by coating test-tube shaped, porous clay supports with alumina sols. These modules exhibited very good permselectivities for applications involving ultrafiltration, as long as several factors were noted when fabricating and using these modules. (1) In order to ensure that the alumina sol was coating the support, rather than being deposited by a slipcasting process, the support was saturated with water before being dip-coated. (2) Coated supports that were mechanically removed from the alumina sol 
displayed better and more reproducible permselectivities than coated supports that were withdrawn from the sol by hand. We employed a removal rate of 16.7 $\mathrm{cm} / \mathrm{sec}$. (3) Application of multiple coats of the alumina sol to the supports was necessary in order to obtain highly permselective modules. However, the supports did not have to be fired at $500^{\circ} \mathrm{C}$ after each coating. Instead, supports could be dipped into sols either 4 or 5 times between each firing at high temperature as long as the coating was dried at $60^{\circ} \mathrm{C}$ for 1 hour between each coating. (4) These modules performed best in permselectivity studies when they were well hydrated. We suspect that this observation would hold for most oxide-based ceramic membrane systems. Thus, users of such systems should consider storing their modules in water when not in use.

Similar modules were also fabricated by coating clay supports with titania sols. The rejection coefficients of the titania-based systems were much lower than those of the alumina modules for low molecular weight polyethylene glycol test solutions. We attribute this result to the formation of cracks in the titania coatings, as observed in scanning electron photomicrographs. Alumina coatings did not display such crack formation. Unsupported titania membranes were also much more likely to crack than were unsupported alumina membrane:s. Note, though, that the titania modules displayed nearly $100 \%$ rejection of bovine serum albumin (molecular weight of 66,000 ).

One advantage of the titania modules was that they were more stable when exposed to solutions at different pH values than were similar alumina modules. Titania modules were essentially unaffected by a 10 week exposure to test solutions maintained between pH values of 2 and 12. Alumina modules, however, suffered severe losses of material during the same 10 week exposure to test solutions at both $\mathrm{pH} 2$ and $\mathrm{pH} 12$. These alumina modules appeared to be quite 
stable when exposed to test solutions that were maintained at $\mathrm{pH}$ values between 4 and 8 . These observations indicate that the $\gamma$-alumina, of which these membranes are comprised, is dissolving at $\mathrm{pH} 2$ and $\mathrm{pH} 12$.

We also observed that the $\mathrm{pH}$ values of test solutions affected the permeate fluxes of these solutions through modules that contained inorganic membranes. In general, it appeared that the highest fluxes were obtained for $\mathrm{pH}$ values near the expected isoelectric points of the materials that comprised the membranes. Changes in flux were much more dramatic on the basic side of the isoelectric point than on the acidic side.

Membrane fouling is a significant problem in most commercial applications of membranes and would be expected to occur with these ceramic membranes as well. We had difficulties with excessive fouling of alumina membranes when performing permselectivity measurements at room temperature on samples of cheese whey. A simple method was developed to clean the alumina membranes during this study (manual cleaning followed by flushing with warm water). Application of this method over a period of 20 days resulted in consistent regeneration of the original permeate flux and permselectivity of these membranes. We expect that more aggressive cleaning procedures could also be used to clean these membranes without causing deletexious changes in their properties. 


\section{References}

1. Anderson, M.A.; Hill, C.G. Jr. Ordered Ceramic Membranes - Annual Progress Report for the Period February 1986 - March 1987. Document DOE/ID/12526-1 (1987).

2. Zeltner, W.A.; Hill, C.G. Jr.; Anderson, M.A. Ordered Ceramic Membranes Annual Progress Report for the Period March 1987 - May 1988. Document DOE/ID/12626-2 (DE89009027), National Technical Information Service, US Dept. of Commerce, Springfield, VA (1988).

3. Anderson, M.A.; Hill, C.G. Jr. Ordered Ceramic Membranes - Annual Progress Report for the Period May 1988 - May 1989. Document DOE/ID/12626-4 (DE89017426), NTIS, Springfield, VA (1989).

4. Anderson, M.A.; Gieselmann, M.J.; Xu, Q. "Titania and Alumina Ceramic Membranes". J. Memb. Sci. 39 (1988) 243-258.

5. Gieselmann, M.J.; Anderson, M.A.; Moosemiller, M.D.; Hill, C.G. Jr. "Physico-Chemical Properties of Supported and Unsupported $\gamma-\mathrm{Al}_{2} \mathrm{O}_{3}$ and $\mathrm{TiO}_{2}$ Ceramic Membranes". Separat. Sci. Technol. 23 [12\&13] (1988) 1695 1714 .

6. Xu, Q.; Anderson, M.A. "Physical-Chemical Properties of $\mathrm{TiO}_{2}$ Membranes Controlled by Sol-Gel Processing". Mat. Res. Soc. Symp. Proc. 132 (1989) 41-46.

7. Xu, Q.; Gieselmann, M.J.; Anderson, M.A. "The Colloid Chemistry of Ceramic Membranes". Polym. Mater. Sci. Eng. 61 (1989) 889-893.

8. Xu, Q.; Anderson, M.A. "Synthesis of Porosity Controlled Ceramic Membranes". J. Mater. Res. 6 [5] (1991) 1073-1081.

9. Xu, Q. "Physical-Chemical Factors Affecting the Synthesis and Characteristics of Transition Metal Oxide Membranes". Ph.D. Thesis, University of Wisconsin - Madison (1991).

10. Brinker, C.J.; Scherer, G.W. Sol-Gel Science: The Physics and Chemistry of Sol-Gel Processing, Academic Press, New York (1990).

11. Gieselmann, M.J.; Anderson, M.A. "Effect of Ionic Strength on Boehmite Hydrogel Formation". J. Amer. Ceram. Soc. 72 [6] (1989) 980-985.

12. Yoldas, B.E. "A Transparent Porous Alumina". Amer. Ceram. Soc. Bull. 54 (1975) 296.

13. Derjăguin, B.V.; Landau, L. Acta Physicochim. USSR 14 (1941) 633; Verwey, E.J.W.; Overbeek, J.Th.G. Theory of the Stability of Lyophobic Cciloids, Elsevier, Amsterdam (1948). 
14. Meakin, P. "Formation of Fractal Clusters and Networks by Irreversible Diffusion-Limited Aggregation". Phys. Rev. Lett. 11 (1983) 119.

15. Kolb, M.; Botet, R.; Jullien, R. "Scaling of Kinetically Growing Clusters". Phys. Rev. Lett. 51 (1983) 1123.

16. Schaefer, D.W.; Martin, J.E.; Wiltzius, P.; Canne11, D.S. Kinetics of Aggregation (F. Family and D.P. Landan, Eds.), North Holland, New York (1984).

17. Hackley, V.A.; Anderson, M.A. "Effects of Short-Range Forces on the LongRange Structure of Hydrous Iron Oxide Aggregates". Langmuir $\underline{5}$ (1989) $191-198$.

18. Hiemenz, P.C. Principles of Colloid and Surface Chemistry, (1st Ed.), Marcel Dekker, Inc., New York (1977).

19. Hunter, R.J. Foundations of Colloid Science, Vol. 1, Oxford University Press, New York (1987) Chaps. $2 \& 7$.

20. Baes, C.F. Jr. and Mesmer, R.E. The Hydrolysis of Cations, John Wiley \& Sons, New York (1976) 150.

21. Chu, L. Unpublished results.

22. Bradley, D.C.; Mehrotra, R.C.; Wardlaw, W. "Structural Chemistry of the Alkoxides. Part I. Amyloxides of Silicon, Titanium, and Zirconium". J. Chem. Soc. (1952) 2027.

23. Matsoukas, T.; Gulari, E. "Monomer-Addition Growth with a Slow Initiation Step: A Growth Model for Silica Particles from Alkoxides". J. Colloid Interface Sci. 132 (1989) 13.

24. McColm, I.J. Ceramic Science for Materials Technologists (L. Hill, Ed.), New York (1983) 276.

25. Peterson, R.A.; Hill, C.G. Jr.; Anderson, M.A. "Permselectivity Characteristics of Supported Ceramic Alumina Membranes". Separat. Sci. Technol. 25 [13-15] (1991) 1281-1293.

26. Moosemiller, M.D. M.S. Thesis, University of Wisconsin - Madison (1988).

27. Kingery, W.D. ; Bowen, H.K.; Uhlmann, D.R. Introduction to Ceramics (2nd ed.), Wiley Interscience, New York (1976) 385.

28. Lonsdale, H.K.; Merten, U.; Riley, R.L. J. Appl. Polym. Sci. $\underline{9}$ (1965) 1341.

29. Baker, E.C.; Mustakas, G.C.; Moosemiller, M.D.; Bagley, E.B. J. Appl. Polym. Sci. 24 (1979) 135.

30. Anderson, M.A.; Xu, Q. U.S. Patent No. 5,006,248 (1991). 

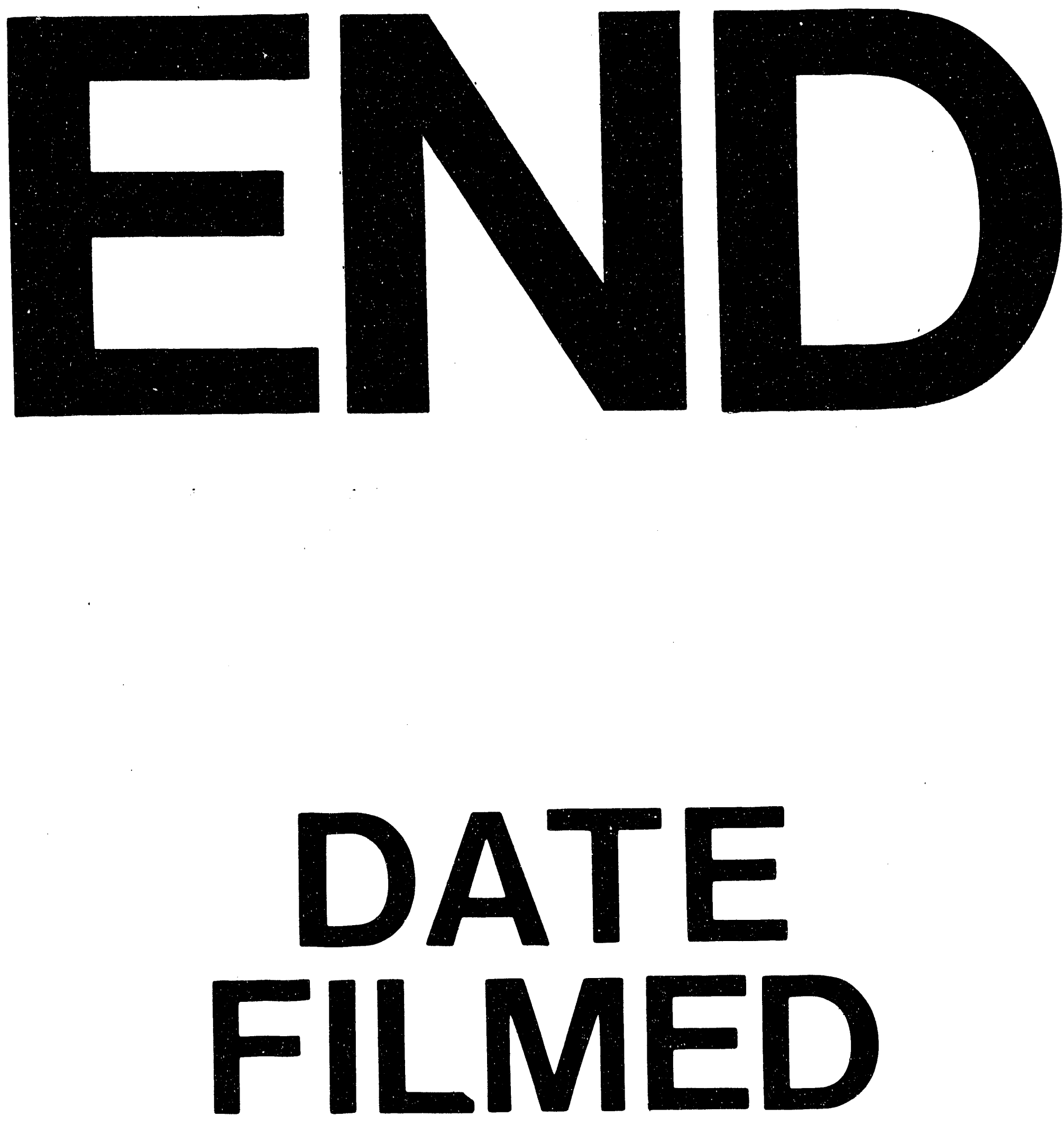

$t$

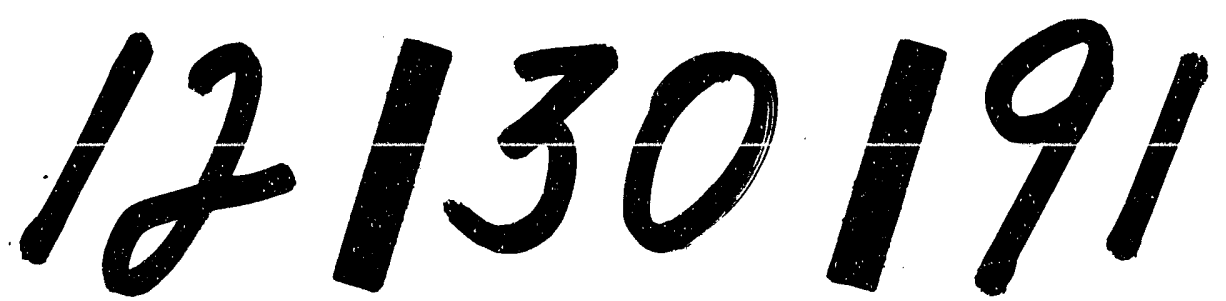


